\title{
Preferential processing in anxiety : selective attention \& spatial affective simon effects
}

Citation for published version (APA):

Schrooten, M. G. S. (2007). Preferential processing in anxiety : selective attention \& spatial affective simon effects. [Doctoral Thesis, Maastricht University]. Datawyse / Universitaire Pers Maastricht. https://doi.org/10.26481/dis.20070628ms

Document status and date:

Published: 01/01/2007

DOI:

10.26481/dis.20070628ms

Document Version:

Publisher's PDF, also known as Version of record

\section{Please check the document version of this publication:}

- A submitted manuscript is the version of the article upon submission and before peer-review. There can be important differences between the submitted version and the official published version of record.

People interested in the research are advised to contact the author for the final version of the publication, or visit the DOI to the publisher's website.

- The final author version and the galley proof are versions of the publication after peer review.

- The final published version features the final layout of the paper including the volume, issue and page numbers.

Link to publication

\footnotetext{
General rights rights.

- You may freely distribute the URL identifying the publication in the public portal. please follow below link for the End User Agreement:

www.umlib.nl/taverne-license

Take down policy

If you believe that this document breaches copyright please contact us at:

repository@maastrichtuniversity.nl

providing details and we will investigate your claim.
}

Copyright and moral rights for the publications made accessible in the public portal are retained by the authors and/or other copyright owners and it is a condition of accessing publications that users recognise and abide by the legal requirements associated with these

- Users may download and print one copy of any publication from the public portal for the purpose of private study or research.

- You may not further distribute the material or use it for any profit-making activity or commercial gain

If the publication is distributed under the terms of Article $25 \mathrm{fa}$ of the Dutch Copyright Act, indicated by the "Taverne" license above, 


\section{Preferential Processing in Anxiety}

Selective Attention \& Spatial Affective Simon Effects 
Colophon

Cover design by Joost Eggermont $@$, www.joosteggermont.nl Production: Datawyse | Universitaire Pers Maastricht

(C) Martien Schrooten, Maastricht 2007

All rights reserved

ISBN 978-90-5278-641-4 


\section{Preferential Processing in Anxiety \\ Selective Attention \& Spatial Affective Simon Effects}

Proefschrift

ter verkrijging van de graad van doctor aan de Universiteit Maastricht, op gezag van de Rector Magnificus, prof. mr. G.P.M.F. Mols, volgens het besluit van het College van Decanen, in het openbaar te verdedigen op donderdag 28 juni 2007 om 10.00 uur door

Martien Germaine Silvie Schrooten

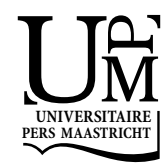




\section{Promotor}

Prof. dr. H.L.G.J. Merckelbach

\section{Co-promotor}

Dr. F.T.Y. Smulders

Beoordelingscommissie

Prof. dr. R.W. Wiers (voorzitter)

Prof. dr. A. Arntz

Prof. dr. P. de Jong (Rijksuniversiteit Groningen)

Prof. dr. K. Mogg (University of Southampton, UK) 


\section{Table of Contents}

1. Preferential Processing in Anxiety: An Introduction 7

1.1. Theoretical Background 9

1.1.1. Anxiety Defined 9

1.1.2. Theoretical Models on Preferential Processing in Anxiety $\quad 10$

$\begin{array}{ll}\text { 1.2. Experimental Paradigms } & 16\end{array}$

$\begin{array}{ll}\text { 1.2.1. Interference Tasks } & 17\end{array}$

$\begin{array}{ll}\text { 1.2.2. Visual Dot-Probe Tasks } & 20\end{array}$

1.2.3. Automatic Preferential Processing? $\quad 42$

1.2.4. Experimental Paradigms: Taken Together 48

1.3. Preferential Processing of Emotional Information as Revealed by

Simon Effects $\quad 50$

1.3.1. Simon Effects Elicited by Preferentially Processed Stimuli $\quad 50$

1.3.2. Spatial Affective Simon Task (SAST) 51

1.3.3. SAST versus Other Paradigms $\quad 52$

2. Outline of the Empirical Chapters 59

3. A Simon Effect for Threat-Related Stimulus Content 61

3.1. Abstract 62

3.2. Introduction $\quad 62$

3.3. Method 65

3.4. Results $\quad 68$

3.5. Discussion 73

4. Biased Processing of Emotional Information in Anxiety: Evidence from Simon Effects

4.1. Abstract 76

4.2. Introduction 76

4.3. Method 78

4.4. Results $\quad 81$

4.5. Discussion 86

5. Preferential Processing of Emotional Stimulus Content in Pathological Anxiety:

Evidence from Simon Effects

5.1. Abstract 90

$\begin{array}{ll}\text { 5.2. Introduction } & 90\end{array}$ 
5.3. Method

5.4. Results 96

$\begin{array}{ll}\text { 5.5. Discussion } & 101\end{array}$

6. Anxiety-Related Attentional Bias and Spatial Response Bias Independently Assessed by a Probe-Classification Task 105

$\begin{array}{ll}\text { 6.1. Abstract } & 106\end{array}$

6.2. Introduction 106

6.3. Method 111

$\begin{array}{ll}6.4 \text {. Results } & 114\end{array}$

6.5. Discussion 122

7. Temporal Dynamics of Selective Attention in Non-Clinical Anxiety 127

$\begin{array}{ll}\text { 7.1. Abstract } & 128\end{array}$

$\begin{array}{lr}\text { 7.2. Introduction } & 128\end{array}$

$\begin{array}{ll}\text { 7.3. Method } & 132\end{array}$

$\begin{array}{ll}\text { 7.4. Results } & 135\end{array}$

$\begin{array}{ll}\text { 7.5. Discussion } & 141\end{array}$

8. General Discussion $\quad 145$

8.1. Review and Discussion of the Empirical Findings 146

8.1.1. Spatial Affective Simon Effects 147

8.1.2. Temporal Dynamics of Selective Attention in Anxiety 150

8.2. Issues in Interpretation 153

8.2.1. Interpretation of Spatial Affective Simon Effects 153

8.2.2. Interpretation of Block Effects 156

$\begin{array}{ll}\text { 8.3. Issues in Methodology } & 158\end{array}$

8.3.1. Stimulus Material 158

8.3.2. SAST Design 161

8.3.3. Attentional Bias and Response Bias Measured in a Single

8.4. Preferential Processing in Anxiety: Future Research 163

$\begin{array}{ll}\text { Summary } & 167\end{array}$

$\begin{array}{ll}\text { Samenvatting } & 171\end{array}$

$\begin{array}{ll}\text { References } & 177\end{array}$

$\begin{array}{ll}\text { Dankwoord } & 190\end{array}$

$\begin{array}{ll}\text { Curriculum Vitae } & 191\end{array}$ 


\section{Chapter 1 \\ Preferential Processing in Anxiety: An Introduction}

This chapter is an adapted and extended version of the Dutch review article: Schrooten, M.G.S., \& Smulders, F.T.Y. (2004). Een experimentele kijk op angst: Aandacht en automatisme in de verwerking van bedreigende stimuli [Anxiety from an experimental point of view: Attention and automaticity in the processing of threatening stimuli]. Nederlands Tijdschrift voor de Psychologie, 59, 135-149. 
As a young researcher in the field of experimental psychopathology, it is hard to imagine that there was a time when emotion was almost written off as a valid topic for theory and research. Nowadays, we are bombarded with theoretical models and experimental studies, all designed in an attempt to get a better understanding of emotions. A highly influential view in this area is the cognitive approach. Proponents of this approach assume that there is a close relationship between our emotions and cognitions. During the last decades, heated debates have been held on the precise nature of this relationship (e.g., Lazarus, 1984, vs. Zajonc, 1984; Oatley \& Johnson-Laird, 1987; LeDoux, 1993). Furthermore, an increasing number of clinical psychologists have become convinced that emotions have a substantial cognitive component and that fundamental biases in information processing may play a critical role in the etiology and maintenance of affective disorders (e.g., stateof-the-art overview in Yiend, 2004a; Johnson-Laird, Mancini, \& Gangemi, 2006). A considerable amount of experimental work has been done on the interface between clinical psychology and basic cognitive psychology. Clinical psychologists have borrowed concepts, theoretical ideas, and experimental methods from cognitive psychologists and more and more hardcore cognitive psychologists have realized the special status of emotionally relevant, salient stimuli in cognitive processing. By now, cognitive models of (disordered) emotions are widely accepted and significant advances have been made in the understanding of how cognitive processes are biased in emotions and affective disorders.

The focus of this doctoral dissertation is on the cognitive concomitants of anxiety. Anxiety has been associated with biases affecting, for example, attention, evaluation, interpretation of ambiguous situations, implicit memory, automatic associations, reasoning, and intrusive ideas (e.g., Mathews \& MacLeod, 2005; Yiend, 2004a). This dissertation project was confined to preferential processing of visual threat-relevant information. In other words, the focus was on the (pre-)attentional priority given to threat in preference to other stimuli in anxiety.

In this introductory chapter, an outline of the theoretical and empirical framework that has guided the research presented in this thesis is provided. A brief review of influential theoretical ideas and models on prioritized information processing in anxiety is presented. Then, an overview of some of the major experimental paradigms used so far to study (automatic) preferential processing of visual emotional stimuli is given, with a particular emphasis on the dot-probe methodology. Variants on the dot-probe task were used in the experiments presented in Chapters 6 and 7 . In the third part of this introduction, a new rationale and paradigm are introduced, developed in order to investigate early preferential processing of emotional information. This new approach was applied in the studies described in Chapters 3-6. 


\subsection{Theoretical Background}

Since the 1960s, with the rise of the cognitive movement in psychology, several theoretical models have highlighted the role of automatic and preferential processing of threat in anxiety. These cognitive models have led to an explosion of systematic experimental research and have, over the years, been refined and modified in order to account for the accumulating empirical data. Before a review is given of the most influential cognitive models in this area, we first consider a definition of "anxiety".

\subsubsection{Anxiety Defined}

Anxiety can be defined as the set of cognitive and behavioral responses to threat. Meanwhile, a categorical distinction has been made between "anxiety" and "fear", based on the presence of the stressor (e.g., Blanchard \& Blanchard, 1990) or based on the direction of the defensive response (e.g. McNaughton \& Gray, 2003). Fear has been linked to actual, localizable threat and active avoidance or escape (fightflight-freezing system). Anxiety, on the other hand, has been associated with potential or diffuse threat, not necessarily currently present or avoidable, and defensive approach tendencies (risk assessment; behavioral inhibition). However, not all researchers in the field have drawn such distinction between fear and anxiety. In scientific literature, as in everyday conversation, the terms "anxiety" and "fear" have been often conflated and used as synonyms. So, "anxiety" has also been used as a collective noun for threat-related responses, independent of type of threat or type of response. In the remainder of this thesis, the term "anxiety" is used in this broad sense, unless specified otherwise.

An important distinction has been made between trait and state anxiety (Spielberger, Gorsuch \& Lushene, 1970; Spielberger, 1972). Trait anxiety refers to general vulnerability or proneness to anxiety, that is the relatively stable tendency of an individual to feel anxious and to perceive a wide range of stimulus situations as dangerous or threatening. State anxiety refers to a transient anxious mood state, that is anxiety felt by an individual at a certain moment in time in a certain (threatening) situation. Endler (1983) refined this idea by stating that an individual's level of state anxiety depends on his or her level of trait anxiety and on the perceived threat. The most-used standardized scale to assess trait and state anxiety is the Spielberger StateTrait Anxiety Inventory (STAI; Spielberger et al., 1970). It has been argued that this questionnaire primarily measures anxiety in social interaction situations, rather than responsiveness to physical threat (Eysenck, 1992; Fox, 1993).

Anxiety levels can become abnormal when intensity and duration of anxious episodes are disproportionate to the risk for harm by a specific object or in a certain 


\section{Chapter 1}

situation, or when such anxious episodes occur without any clear threat or problem. People suffering from these symptoms meet the criteria for an anxiety disorder if their fears or concerns also interfere and cause distress in their lives. As outlined in the Diagnostic and Statistical Manual of Mental Disorders (4th ed. rev., DSM-IV-R; American Psychiatric Association, 2000), anxiety disorders include generalized anxiety disorder (GAD), social anxiety disorder (social phobia), specific (simple) phobia, panic disorder, obsessive-compulsive disorder, and post-traumatic and acute stress disorder (but excluding substance-induced anxiety or anxiety due to a medical condition). For the exact diagnostic criteria, prevalence, and course of these disorders, the interested reader is referred to the DSM-IV-R.

\subsubsection{Theoretical Models on Preferential Processing in Anxiety}

Some cognitive models on anxiety have been strongly influenced by clinical observations, whereas other models have their roots in more evolutionary or biological considerations. For a detailed overview of the most influential theoretical ideas and accounts on cognitive biases in anxiety, the reader may consult, for example, Eysenck (1992), Mogg and Bradley (1998), and Mathews and Mackintosh (1998).

Beck's schema theory (1976) and Bower's network model (1981) predicted that all emotions would be associated with emotion-congruent biases in all aspects of cognitive processing (selective attention, memory, stimulus evaluation etc.). Beck, one of the founding fathers of cognitive therapy, suggested, for example, that anxiety is characterized by dysfunctional schemata (structures of long-term memory representations) related to threat. Activation of such cognitive representations would result in selective processing of anxiety-congruent information. According to Beck, individual differences in (sensitivity or activity of) threat-related schemata underlie vulnerability to clinical anxiety. At around the same time, Bower developed a model in which each emotion is represented as a node in an associative network in memory. In this model, activation of, for example, the anxiety node spreads in the network to other nodes (concepts) that are linked to it. This would result in increased accessibility of these related concepts and in prioritized processing of information related to anxiety, danger or threat. These early theories have been criticized in the eighties, in that they could not explain empirical findings suggesting that in anxiety, there is no pervasive bias throughout all cognitive processes. For example, these models could not explain the failure to find evidence of an anxiety-related recall or recognition bias (e.g., Mogg, Mathews, \& Weinman, 1987).

In the years that followed, several cognitive models were developed, all emphasizing the crucial role of selective attention in anxiety (e.g., Williams, Watts, MacLeod, \& Mathews, 1988; 1997; Eysenck, 1992; Oatley \& Johnson-Laird, 1987). Williams and colleagues $(1988,1997)$ outlined a processing stage model and 
argued that (1) anxiety is primarily associated with selective attentional bias for threat; that is a tendency to attend a threat-related stimulus rather than a neutral stimulus that is presented concurrently; (2) this bias operates automatically, at a very early stage of processing, prior to awareness; (3) such (pre-)attentional bias for threat plays a primary role in causing anxiety; (4) individual differences in the direction of this bias underlie vulnerability to anxiety. At the first pre-attentive stage of this influential model, the emotional value of incoming stimulus information is computed by an Affective Decision Mechanism (ADM; a connectionist network in the 1997 version of the model). If the value of the incoming stimulus is decided to be threatening enough, a second mechanism is activated, allocating attentional resources towards this stimulus (Resource Allocation Mechanism; RAM). Whereas state anxiety would affect the output of the ADM, trait anxiety would influence activation of the RAM. According to this model, high-trait-anxious individuals are characterized by an attentional bias towards threat-related information (vigilance'), whereas low-trait-anxious direct their attention consistently away from threat (avoidance). In case of highly threatening stimuli, high-trait-anxious individuals would become even more vigilant for these stimuli, but low-trait-anxious persons more avoidant (trait-state interaction hypothesis). This latter prediction is rather counter-intuitive and has been criticized several times, on empirical as well as theoretical grounds (see below; e.g., Mogg \& Bradley, 1998; Mathews \& Mackintosh, 1998; Mogg, McNamara et al. 2000; Wilson \& Macleod, 2003). Another prediction made by Williams and colleagues is that high-trait-anxious individuals would initially direct their attention towards threat, but would subsequently try to direct their attention away from it. Such a vigilance-avoidance pattern of attentional processing may help to reduce anxious states, by avoiding deep processing of threatrelated information, but may also prevent objective evaluation of threat or habituation to it.

The idea that selective attention plays an important role in anxiety makes sense also in light of the assumed adaptive function of anxiety. Oatley and Johnson-Laird (1987) argued that basic emotions have evolved to serve particular biological and social communicative functions. From this evolutionary perspective, it is expected that different emotions, with different functions, are characterized by quite different patterns of cognitive processing, rather than by general bias effects as was predicted by Beck (1976) and Bower (1981). It is likely that the key function of anxiety is early, prioritized detection of potential threat in the environment and rapid, adap-

\footnotetext{
${ }^{1}$ Typically, the term vigilance is used to refer to sustained attention or tonic alertness. In the present literature, however, the term refers to preferential attentional processing in favor of a certain (e.g., threat-related) stimulus.
} 
tive responding if necessary (Oatley \& Johnson-Laird, 1987). Also attention has been related to preferential processing of salient or relevant information. For instance, it has been assumed that attentional processes facilitate identification of critical stimuli and maintain processing resources on them (e.g., LaBerge, 1995; Pashler, 1998). Therefore, it seems very plausible that attentional processes are critically involved in anxiety (Oatley \& Johnson-Laird, 1987; Eysenck, 1992).

Evolutionary considerations are also central to Öhman's theoretical views on anxiety (Öhman, 1993, 1996, 2000; Öhman \& Mineka, 2001). In his accounts, the automatic capture of biological threat and the automatic activation of fear are emphasized. He assumed that, at a very early stage of processing, before the stimulus has entered awareness, threat feature detectors analyze incoming stimulus information. This information is passed on to a non-conscious system evaluating the significance of the output of these detectors, and subsequently to a conscious perception system that is connected to an expectancy system. If the incoming information is related to biologically or evolutionarily relevant threat or if it has a highly intense threat value, the feature detectors immediately activate autonomic arousal, which in turn facilitates behavioral responses to threat, and the threat-related stimuli will receive greater priority for further processing (e.g., for access to selective attention). Other information is passed on to the conscious perception system. If stimuli, after more conscious appraisal, are perceived to be (potentially) threatening (e.g., threat words), autonomic arousal is also activated via this slower route. The sensitivity of the significance evaluating system is influenced by autonomic arousal and the expectancy system. Furthermore, these systems for threat detection and fear elicitation are thought to be especially sensitive in fearful individuals (Öhman, Flykt, \& Esteves, 2001).

Öhman's ideas were largely based on evidence from psychophysiological and neuropsychological research. For example, he and his colleagues measured autonomic activity in response to fear-relevant (e.g., spiders) and fear-conditioned stimuli, even when these stimuli were presented under conditions of restricted awareness (Esteves, Dimberg, \& Öhman, 1994; Öhman \& Soares, 1993, 1994, 1998). These data suggest that autonomic fear responses can be evoked by perceptually degraded stimuli that were only pre-attentively analyzed (however, see also Mayer, Merckelbach, De Jong, \& Leeuw, 1999). The latter effect with fear-conditioned stimuli was only evident for evolutionarily threat-relevant stimuli (e.g., snakes, spiders, angry faces), but not for other, more positive stimuli conditioned to threat (e.g., flowers, happy faces; Öhman \& Soares, 1993). This finding is in line with Seligman's (1971; Öhman \& Mineka, 2001) "preparedness" theory of fear learning, suggesting that we are genetically prepared to make associations between aversive stimuli and stimuli that were potentially threatening for our ancestors. It is likely to assume that the attention system of anxious people might be especially sensitive to biologically relevant fear stimuli. 
Extensive neuro-biological research has established that the key structure of the fear system is situated in the amygdala, two almond-shaped sub-cortical brain structures (e.g., LeDoux, 1992, 1995; Davis, 1992 a, b; reviews by e.g., Armony \& LeDoux, 2000; Davis \& Whalen, 2001). The amygdala has been found to play an important role in fear conditioning, autonomic and hormonal changes, motor responses and attentional processes. Furthermore, this fear system is especially sensitive to stimuli that may have imposed threat to our distant ancestors (e.g., LeDoux, 1996; Morris, Öhman \& Dolan, 1998). In short, experimental research suggests two neural pathways to be involved in fear (for a review see e.g., Compton, 2003). Via a direct route with the thalamus, the amygdala receives low-level perceptual information, and subjects it to a quick-and-dirty analysis in order to evaluate its emotional significance (e.g., LeDoux, 1996; Whalen et al., 1998; Morris et al., 1998). For example, several studies have shown that the amygdala is responsive to threat-related information (e.g., angry or fear-conditioned faces) even when they are presented under conditions of restricted awareness (Morris et al, 1998; Morris, Öhman, \& Dolan, 1999; Whalen et al, 1998). Besides this fast pathway, there is a second thalamo-cortico-amygdala route, involved in more complete cognitive processing of the stimuli. This slower, conscious, more detailed processing at the cortical level is followed by several complex cortico-limbic (cognitive-emotional) interactions (e.g. LeDoux, 1996). Gray and McNaughton (2003) argued that the amygdala is also the central brain structure underlying more generalized anxiety, but that its interconnections with the septo-hippocampal system are crucial for the specific cognitive components associated with anxiety (detect and resolve conflict between concurrent goals).

Öhman's model and the biological models of fear and anxiety described above share the idea that there are some specialized mechanisms that respond automatically and pre-attentively to evolutionary threat stimuli, without any (direct) strategic control. Wells and Matthews (1994; Matthews \& Wells, 2000), however, argued that anxiety-related biases in preferential processing critically depend on top-down control processes related to individual plans, goals and expectations. They postulated a Self-Regulatory Executive Function (S-REF), setting the goals for controlled processing based on the difference between actual and desired states. Normally, $S$ REF operates for a relatively short period, for in general individuals are able to select a successful self-regulatory strategy when, for example, confronted with a stressor. However, those vulnerable to anxiety and those with an anxiety disorder fail to adopt an adequate coping strategy and the S-REF keeps on trying. As a result, the person continues to worry and ruminate about personal problems. Wells and Matthews argued that anxiety-related attentional bias effects depend on a strategy for coping with threat stimuli. Such a strategy is initiated voluntarily and might also influence responses to stimuli that are not consciously perceived. 


\section{Chapter 1}

Difference in coping style is also an important aspect of Eysenck's (1997, 2000) Four-Factor Theory of anxiety. Eysenck stated that anxiety depends on four different sources of information (external stimuli, internal physiological stimuli, own behavior, and own cognitions) and that the effect of this information on experienced anxiety depends on attentional and interpretative processes. According to this theory, high-trait-anxious persons (high in self-reported trait anxiety, low in defensiveness), repressors (low in self-reported trait anxiety, high in defensiveness) and truly low-trait-anxious persons (low in self-reported trait anxiety, low in defensiveness) differ with respect to their processing of this information, especially when under stress. Whereas high-anxious persons exhibit cognitive biases leading them to exaggerate the threat value of this information, repressors have biases leading them to minimize it (i.e., a repressive coping style). Truly low-anxious persons have no attentional or interpretative biases at all. Eysenck argued that GAD is highly similar to trait anxiety and patients suffering from this disorder have, in principle, cognitive biases for all four sources of information. He explained the existence of more specific anxiety disorders by stating that in each of these disorders cognitive processing is biased for only one source of information. For example, panic patients have cognitive biases mainly for their own physiological activity, and patients with a specific phobia for certain external stimuli in the environment (Eysenck, 2000).

In 1998, two further cognitive models on anxiety were formulated, both building on the aforementioned theoretical ideas, and both trying to give a better account for the observed empirical data: the cognitive-motivational analysis of anxiety by Mogg and Bradley (1998) and the cognitive model of selective processing in anxiety by Mathews and Mackintosh (1998; see also Mackintosh \& Mathews, 2003). Both groups leveled similar criticisms regarding the cognitive model proposed by Williams and colleagues (1988, 1997; see above). Their criticism focused on the counterintuitive prediction that low-trait-anxious individuals would become increasingly more avoidant from threat stimuli as threat value increases, and the assumption that attentional bias plays a primary role in causing anxiety. Both groups suggested the existence of some intensity or urgency threshold above which attention is addressed towards the stimulus, and below which attention is drawn away. Individual differences in attentional bias would reflect a switch from avoidance to vigilance at some lower level of perceived threat in those vulnerable to anxiety. The level at which this transition occurs depends on how vulnerable an individual is to anxiety and on the specific state conditions. Low-trait-anxious would only direct their attention towards highly threatening stimuli, but not to stimuli that are only mildly threatening. Both models suggested that individual differences in trait anxiety can arise from biases in stimulus evaluation (overestimation of threat value), rather than (only) from biases in selective attention. However, biases in selective attention would contribute to the maintenance of anxiety. 
In Mogg and Bradley's (1998) cognitive-motivational view of anxiety, anxiety is defined as an aversive motivational state, characterized by some biased cognitive processing, allowing rapid detection and efficient responding in case of threat. In their model, the threat value of incoming information is first assessed by a valence evaluation system. The output of this system depends, of course, on the specific stimulus input, but also on the specific situation, prior learning experiences, biological preparedness, and state anxiety. In addition, individual differences in trait anxiety would reflect differences in the reactivity of this system to threat stimuli. The output of the valence evaluation system is passed on to a goal engagement system, determining the allocation of resources for action and cognitive processing. Stimuli perceived as threatening would result in (automatic) interruption of current goals, as well as in behavioral and attentional orienting towards threat. In contrast, if the incoming information is not perceived as (potentially) threatening, attention would remain preferentially on ongoing activities, current goals would be pursued, positive stimuli prioritized, and minor negative stimuli ignored.

In Mathews and Mackintosh's (1998) model, multiple stimuli are processed in parallel and compete for attentional resources. The size and direction of attentional bias effects depend on the interaction between (1) bottom-up activation of threatrepresentations by a threat evaluation system (TES; e.g., amygdala, hippocampus) and (2) top-down activation of neutral representations related to other goals by an attentional control system (e.g., anterior cingulate and dorsolateral prefrontal cortex). In case of anxiety, when a stimulus related to (potential) threat is encountered, this stimulus is matched to threat representations, and this will result in the allocation of attention to this stimulus. Such bottom-up activation can be counteracted by topdown attentional control (e.g., favoring goal/task-related stimuli), but this strategic control can, in turn, be overruled by cognitive load or state anxiety. Furthermore, Mathews and Mackintosh stated that the detection of threat can occur automatically, at non-conscious levels. Following this early detection, it might be that multiple meanings of the stimulus are activated, and interpretation biases depend on a similar competition as described above for detection, but now between multiple meanings.

The final model described here, is the model recently outlined by Fox on the specific nature of attentional mechanisms associated with anxiety (Fox, 2004; Fox, Russo, Bowles, \& Dutton, 2001). From cognitive psychology, it is known that selective attention can be broken down in separate components, for example engagement, shifting, and disengagement (Posner \& Petersen, 1990). In Fox's framework, fear and specific phobia are characterized by initial orienting of attention towards the source of threat, followed by attentional avoidance. Such cognitive avoidance would prevent habituation or objective evaluation of potentially threatening stimuli. More generalized anxiety and trait anxiety, in contrast, are associated with initial attentional orienting towards potential threat, followed by a tendency to 


\section{Chapter 1}

maintain attention on this information (i.e., delayed disengagement). This tendency to dwell on threat-related information may serve to maintain and enhance anxiety states.

In the previous paragraphs, the most influential theoretical ideas and models on preferential processing in anxiety were summarized. ${ }^{2}$ Several important issues put forward by these accounts have already been addressed in experimental research. For example, research has focused on the precise nature of selective attentional bias in anxiety, its content specificity, its automaticity (e.g., role of stimulus awareness), its time-course (vigilance avoidance/maintenance), the relative roles of trait and state anxiety, differences or similarities between non-clinical and clinical anxiety, the effects of repressive coping style and attentional control, and recently, research also started to investigate the causal role of attentional bias effects in anxiety. Some psychophysiological and neuropsychological data were already briefly mentioned above. The emphasis of the present thesis is, however, on behavioral data. Several reaction time tasks have been developed to measure preferential processing of emotional information. In the next section, a brief overview of some of the major behavioral paradigms used in this area and a review of some typical experimental studies, findings and insights so far are given. Following this, a rationale and description of a novel reaction time paradigm are presented, developed to provide converging evidence for early preferential processing in anxiety.

\subsection{Experimental Paradigms}

As mentioned above, concepts and paradigms have been borrowed from basic cognitive research to investigate cognitive processing of emotionally relevant information. Modified versions of interference tasks, spatial cueing tasks, and search tasks have been employed to study preferential processing in anxiety. In the following paragraphs, these tasks are discussed successively (for a detailed description of the original paradigms, see Luck \& Vecera, 2002) and data gathered by using these different tasks are described. The focus of the present dissertation work was on preferential processing of visual information, and therefore, only research in the visual domain is reviewed here. The overview is limited to studies that included a group of adult participants, with a clinically diagnosed anxiety disorder or with high self-reported anxiety.

\footnotetext{
${ }^{2}$ More specific cognitive models have been developed to account for different forms of pathological anxiety. An overview of these models is, however, beyond the scope of the present introduction; the interested reader is referred to Mathews and MacLeod (2005).
} 


\subsubsection{Interference Tasks}

During interference tasks, participants perform a primary task and at the same time, they also have to ignore some distracting information. The underlying idea is that selective processing of task-irrelevant distractors would disrupt the performance on the main task.

During the Stroop color-word task, for example, participants are asked to name the print color of color words, but to ignore the meaning of these words (Stroop, 1935; for a review, see MacLeod, 1991). During a control condition, participants name the ink color of meaningless stimuli (e.g., a series of Xs or a color patch). Typically, color naming is slowed down when the ink color and the meaning of the word are incongruent (e.g. "yellow" in green ink) compared to color naming in the control condition. When the print color and the meaning of the word are congruent (e.g. "yellow" in yellow ink), color naming is often faster than naming in the control condition. This facilitation is usually much less than the corresponding interference in the incongruent condition (MacLeod, 1991). The classical Stroop effect is the difference in color-naming performance between congruent and incongruent wordcolor conditions.

In the emotional variant of the Stroop task, color words are replaced by emotional and emotionally neutral words, and again, participants' task is to name the color the words are printed in while ignoring the meanings of the words (for reviews, see Williams, Mathews, \& MacLeod, 1996; MacLeod, 2005). The emotional Stroop interference effect refers to slower and less accurate color naming when emotional words are presented than when neutral words are presented. The size of this effect has often been taken as an index of the degree to which attentional resources are captured by word content. Larger emotional Stroop interference effects in emotionally disturbed individuals compared to matched controls have been taken as evidence for selective attentional bias in the emotional group.

Emotional Stroop experiments have demonstrated that anxious, compared to non-anxious, individuals are slower (and less accurate) in naming the color of words related to their worries, object of fear, or relevant threat than in naming the color of other words (for an overview, see e.g., Williams et al., 1996). Such content-specific interference effects have been observed in a wide range of anxiety disorders, including GAD (e.g., Mathews \& MacLeod, 1985; Mogg, Mathews, \& Weinman, 1989; Mogg \& Bradley, 2005), panic disorder (e.g., Hope, Rapee, Heimberg, \& Dombeck, 1990), social phobia (e.g., Hope et al., 1990; Mattia, Heimberg, \& Hope, 1993), spider phobia (e.g., Lavy, 1993; Watts, McKenna, Sharrock, \& Treazise, 1986), post-traumatic stress disorder (e.g., McNally, Kaspi, Riemann, \& Zeitlin, 1990), and obsessive compulsive disorder (e.g., Tata, Leibowitz, Prunty, Cameron, \& Pickering, 1996). Emotional Stroop effects have not only been observed in 
pathological anxiety, but also in individuals with high levels of self-reported trait anxiety, especially when under stress (e.g., MacLeod \& Mathews, 1988; Mogg, Mathews, Bird, \& MacGregor-Morris, 1990; Richards, French, Johnsson, Naparstek, \& Williams, 1992; MacLeod \& Rutherford, 1992; Mogg, Kentish, \& Bradley, 1993). Furthermore, emotional Stroop effects in clinical anxiety disappeared after cognitive therapy (Mattia et al., 1993; Lavy, 1993; Mathews, Mogg, Kentish, \& Eysenck, 1995). This observation suggests that emotional Stroop effects were mainly due to some transient state variable, like anxious mood, or to an interaction between state and trait anxiety. In addition, emotional Stroop effects have been found to be suppressed instead of enhanced in intense threatening situations; for example, in snake phobics when exposed to a real snake or spider (Mathews \& Sebastian, 1993), in social phobics just before they had to give an oral presentation (Amir, McNally, Riemann, Burns, Lorenz, \& Mullen, 1996), and in individuals with an abnormal fear for dental treatment when exposed to dental sights and sounds (Muris, Merckelbach, de Jong, 1995, but see Jones, Stacey, \& Martin, 2002). One explanation given for the rather paradoxical finding of suppressed Stroop effects in more intense, threatening situations is that under such conditions top-down inhibitory control is strong, blocking attention for word stimuli, and giving processing priority to more severe threat in the environment (Mathews, 2004; Mathews \& Mackintosh, 1998). Finally, some emotional Stroop studies report interference effects for positive words in anxiety, but only for positive words related to concerns or threat (e.g., antonyms of threat-words; Mathews \& Klug, 1993; Martin, Williams, \& Clark, 1991).

Although emotional Stroop effects have been frequently observed and are considered to be reliable and robust, their interpretation is far from clear. Emotional Stroop effects might, as originally proposed, be due to selective attention towards emotional word content. However, it has been suggested that these effects might also be interpreted in terms of later cognitive avoidance, focused attention, response inhibition, and / or delay in disengaging attention (e.g., de Ruiter \& Brosschot, 1994; Fox, 2004; Williams et al., 1996). Often, accounts for classical Stroop effects have been (tacitly) applied to emotional Stroop effects (e.g. Williams et al., 1996). However, also for classical Stroop effects, there is a debate about the processes involved (e.g., processes at the level of response selection and / or perceptual encoding; De Houwer, 2003; for an overview of theoretical accounts, see MacLeod, 1991). Furthermore, caution is needed when generalizing explanations from classical to emotional Stroop effects. Although the emotional Stroop task is at first sight similar to the original Stroop word-color task (i.e., naming print color, ignoring word meaning), these tasks differ structurally from each other (De Houwer, 2003; Algom, Chajut, \& Lev, 2004; McKenna \& Sharma, 2004). In classical Stroop tasks, the congruence between word content and a task-relevant stimulus feature (and the response to it) is manipulated. In emotional Stroop tasks, however, there is no 
logical relationship of congruence or incongruence between emotional word content and other stimulus features or responses. This fundamental difference in structure led researchers to conclude that classical and emotional Stroop effects are independent and unrelated phenomena (Algom et al., 2004; Chajut, Lev, \& Algom, 2005; but Dalgleish, 2005) and likely reflect the operation of qualitatively different mechanisms (De Houwer, 2003; Algom et al., 2004). So, accounts for classical Stroop effects (e.g., conflicts in response selection; Cohen, Dunbar, \& McClelland, 1990) do not necessarily hold for emotional Stroop effects (De Houwer, 2003).

In the above explanations of emotional Stroop effects, it has been assumed that these effects reflect some processing bias in favor of distractors with an emotional content. Alternatively, it might be that threat-related and neutral distractors are processed to the same degree, but that the presence of threat-related words elevates a negative affective state or arousal to a level where it disturbs performance (MacLeod, Mathews, \& Tata, 1986). It might be that interference effects reflect the operation of a defense system temporarily disrupting all ongoing activity (e.g., inhibition or less processing resources for threat-irrelevant stimuli; Algom et al., 2004).

In sum, in numerous studies, larger emotional Stroop interference effects have been observed for threat-relevant words, as opposed to neutral words, in anxious groups compared to low-anxious groups. However, it remains unclear exactly which mechanisms are involved.

The emotional variant of the classical Stroop task is the most widely used, but not the only interference task in anxiety research. Versions of the separated Stroop or flanker task have also been employed (e.g., Fox, 1993, 1994, 1996, 2004; Mathews, May, Mogg, \& Eysenck, 1990; Eysenck \& Byrne, 1992; Lavy, van den Hout, \& Arntz, 1993). In contrast to the emotional Stroop task, in these tasks tobe-attended and to-be-ignored information are presented at spatially separate locations. That is, participants are instructed to respond to a target stimulus (e.g., name the color of a color patch or classify a number as odd/even) and to ignore other stimuli (e.g. neutral or emotional words) flanking the target. Therefore, these tasks provide a more appropriate test of selective processing than the emotional Stroop task, during which to-be-attended and to-be-ignored information are integrated in one single stimulus (Fox, 1993). By applying these interference tasks, it has, for example, been observed that high-anxious individuals take longer to name the color of the target, when at the same time also threat-related words, as opposed to neutral words, are presented, even when this distracting information is presented outside the focus of attention (high-trait-anxious: Fox, 1993; Eysenck \& Byrne, 1992; (recovered) GAD patients under conditions requiring visual search: Mathews et al., 1990). In addition, high-trait-anxious people and patients with GAD seem to be characterized by a general inability to maintain attentional focus and resist distraction (Fox, 1993; Eysenck \& Byrne, 1992; Mathews et al., 1990). 


\section{Chapter 1}

So far this review has focused on findings from studies showing that in anxiety, performance on interference tasks is more disturbed when threat-related rather than neutral distractors are presented. Such impaired performance might be the result of some processing bias in favor of threat. However, as mentioned above, this disturbed performance might, for example, also be the consequence of heightened levels of arousal, especially when threat-related information is always presented into attentional focus. To circumvent this interpretative problem, and in an attempt to get a better measure of the spatial distribution of selective attention in anxiety, tasks have been developed during which an emotional and a neutral stimulus are presented simultaneously and during which the deployment of attention is assessed by responses to a neutral target. In these tasks, selective bias effects are not derived exclusively from impaired performance, but from the difference in response latency between conditions during which the target appears at the location of the emotional stimulus and conditions during which the target appears at the location of the neutral stimulus. The attentional deployment task most widely applied in anxiety research is the visual dot-probe task (MacLeod et al., 1986). Some modified versions of this task were applied in the experiments described in Chapters 6 and 7. Previous dot-probe research was also an important source of inspiration for the other studies presented in this thesis. The dot-probe task and research in which this task was used are discussed in the following section. For alternative paradigms assessing the deployment of selective attention, see for example Gotlib, McLachlan, and Katz (1988; Mogg et al., 1991), and Christie and Klein (1995).

\subsubsection{Visual Dot-Probe Tasks}

During dot-probe tasks, at critical trials, one neutral and one emotional word or picture are presented at two fixed positions on the screen (at the left and right, or above and below central fixation). The emotional stimulus appears with equal probability in either location. After, for example, half a second, the stimulus pair disappears and a probe (e.g. a small dot) is presented at the position previously occupied by the emotional stimulus or at the other position, with equal probability. ${ }^{3}$ In basic cognitive psychology research, spatial cueing studies have consistently shown that responses are faster to probes appearing at attended rather than unat-

\footnotetext{
${ }^{3}$ In the modified dot-probe task as used by Mansell and colleagues (Mansell, Clark, Ehlers, \& Chen, 1999; Chen, Ehlers, Clark, \& Mansell, 2002; Mansell, Ehlers, Clark, \& Chen, 2002), there are four, instead of two, possible positions on the screen. The stimulus pairs are presented diagonally (top left/bottom right or bottom left/top right) and the positions of these stimuli inform about the two possible locations at which the following probe can occur. In typical dot-probe tasks, the stimulus pair always appears at the same locations, and are entirely irrelevant to the task.
} 
tended locations (e.g. Posner, Snyder, \& Davidson, 1980). Based on this finding, in dot-probe studies, faster responses when emotional stimulus and probe appear in the same spatial position than when they occur in different positions have been interpreted as reflecting attentional bias for the emotional stimulus; that is, selective attention for the emotional stimulus, in preference to a simultaneously presented neutral one.

In the dot-probe literature, it is common to compute an attentional bias index reflecting the magnitude and direction (i.e., attention on the emotional stimulus or on the neutral stimulus paired to it?) of the bias. Attentional bias scores are typically calculated by subtracting the reaction time when emotional stimulus and probe occur in the same position from the reaction time when they appear in different positions. Calculated in this way, positive values reflect faster responses on spatially corresponding than on non-corresponding trials, thereby indicating selective attention for the emotional rather than the neutral stimulus. The size of attentional bias effects as measured with the dot-probe task is in the order of milliseconds.

Since its first application, the dot-probe task has been modified in several ways. To start with, three basic types of the task can be distinguished based on the response that is to be given: simple detection of the probe, or forced choice based on the position or the type of the probe (Mogg \& Bradley, 1999a; Salemink, van den Hout, \& Kindt, 2007). In addition to response position, other parameters have been (systematically) varied in order to address several issues put forward by cognitive models on anxiety. Different types of stimuli have been presented, different exposure durations used and different groups of participants tested in order to study, for example, the content specificity of attentional bias in anxiety, its dynamic time-course, supposed automaticity, and the effects of threat intensity level of the stimuli (for a review, see for example, Mogg \& Bradley, 1998; 2004). Table 1.1. presents a summary of dot-probe studies on selective attention in anxiety. 


\section{Chapter 1}

Table 1.1. Review of dot-probe studies in anxiety research. The review is limited to studies with adult participants, trait anxiety, state anxiety, social anxiety, GAD, panic disorder, social and specific phobia, and does not pretend to be complete. Only reaction-time results are reported.

\section{How to read Table 1.1 .}

Table 1.1. has to be read from the left to the right. Information on the same line belongs together. If no information is given, see information on previous line same column.

Abbreviations (organized per column, alphabetically):

Reference: author + year of publication (1986-2007), chronologically

$\exp$ experiment

Task:

C probe-classification or -differentiation task

D probe-detection task

DC probe-detection task + reading aloud digit appearing between the stimuli

DT probe-detection task + reading aloud word at top position

P probe-position task

Participants: number of participants + groups tested and/or continuous measure (questionnaire) used to define groups or used in correlational/regression analyses

italic state anxiety manipulation

underlined if more than two groups, underlined letters are used in attentional bias columns to specify the groups

across groups as defined in lines above taken together

C healthy control group

D high levels of defensiveness or social desirability

GAD generalized anxiety disorder

HHA high-health-anxious (subclinical; self-reported)

HSA high-state-anxious (subclinical; self-reported)

HTA high-trait-anxious (subclinical; self-reported)

LHA low-health-anxious (subclinical; self-reported)

LSA low-state-anxious (subclinical; self-reported)

LTA low-trait-anxious (subclinical; self-reported)

PD panic disorder

physical worries anxious thoughts about physical health concerns (illness, death, accident)

R

repressor (low-trait-anxiety + high levels of defensiveness/ or social desirability)

S unselected students

SNAQ snake anxiety questionnaire

social worries anxious thoughts about social concerns (being criticized, socially evaluated)

SP (generalized) social phobia

SPQ fear of spiders questionnaire

STAI-state Spielberger State-Trait Anxiety Inventory state subscale

STAI-trait Spielberger State-Trait Anxiety Inventory trait subscale

V unselected volunteers from community sample

Critical Stimuli: definition of emotional stimuli; all emotional stimuli paired to neutral stimulus from same category, unless stated otherwise 


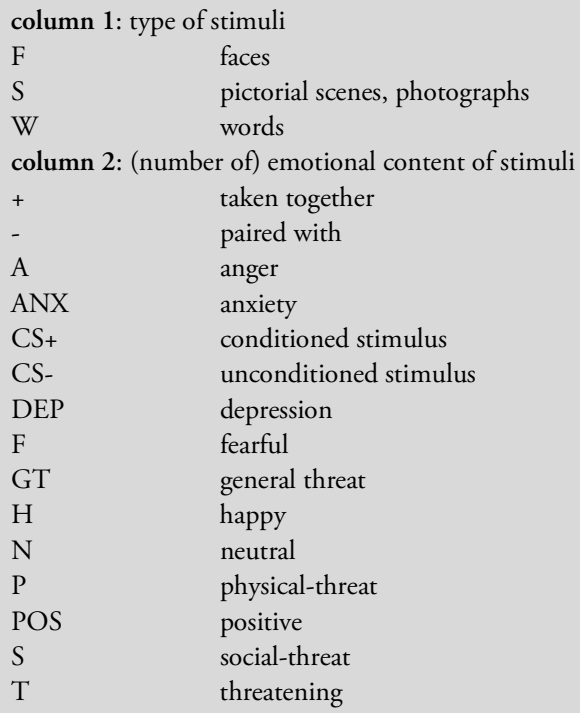

Filler: (number of) stimuli used as filler material; proportion of all stimuli

$\begin{array}{ll}- & \text { no fillers } \\ \text { A-A } & \text { anger-anger pairs } \\ \mathrm{H}-\mathrm{H} & \text { happy-happy pairs } \\ \mathrm{N} & \text { neutral-neutral pairs } \\ \text { POS } & \text { positive-neutral pairs }\end{array}$

Probed: (number of) stimuli followed by a probe

A all pairs (critical + filler)

AC all critical pairs

C critical pairs

F filler pairs

Exposure: in milliseconds (ms); exposure conditions presented intermixed, unless stated otherwise across exposure conditions as defined in lines above taken together B blank screen

BG between-group manipulation

BU build-up time

M mask

SOA: interval between onset of the stimulus pair and onset of the probe (stimulus onset asynchrony); in milliseconds (ms)

Attentional bias: attentional bias score = (mean reaction time when critical stimulus and probe do not spatially correspond minus mean reaction time when critical stimulus and probe correspond); in milliseconds $(\mathrm{ms})$; bias scores and statistics as reported in published article

HA: attentional bias score high-anxious group

LA: attentional bias score low/non-anxious group

* $\quad$ bias significantly different from zero (no bias) $(p$ 2-tailed $\leq .05)$

; $\quad$ task half 1 ; task half two 


\section{Chapter 1}

$\begin{array}{ll}\text { bl. } & \text { task block } \\ \text { LVF } & \text { critical stimulus in left visual field } \\ \text { RVF } & \text { critical stimulus in right visual field } \\ \text { t } & \text { bias trend-level significantly different from zero }(.05<p \text { 2-tailed }<.1)\end{array}$

nb. 1: HA and LA groups are defined in column Participants

nb. 2: if only continuous measure given in column Participants, then groups were based on median split of scores on this measure

nb. 3: if more than two groups, underlined letters indicate group; see column Participants

nb. 4: if no groups, bias score for the sample as a whole is presented in the middle of HA and LA columns

HA vs. LA: statistics comparing scores of high-anxious and low-anxious groups

- $\quad$ versus

BG overall between-group effect when more than two groups

Continuous: statistics of correlational or regression analyses with continuous measure as defined in column Participants

\begin{tabular}{ll}
$*$ & significant correlation $(p 2$-tailed $\leq .05)$ \\
half & task half $(1$ or 2$)$ \\
$n s$ & non-significant \\
$r$ & correlation \\
state curvil. & curvilinear effect of state anxiety \\
trait curvil. & curvilinear effect of trait-anxiety \\
\multicolumn{2}{l}{ trait x state interaction effect of trait- and state-anxiety }
\end{tabular}




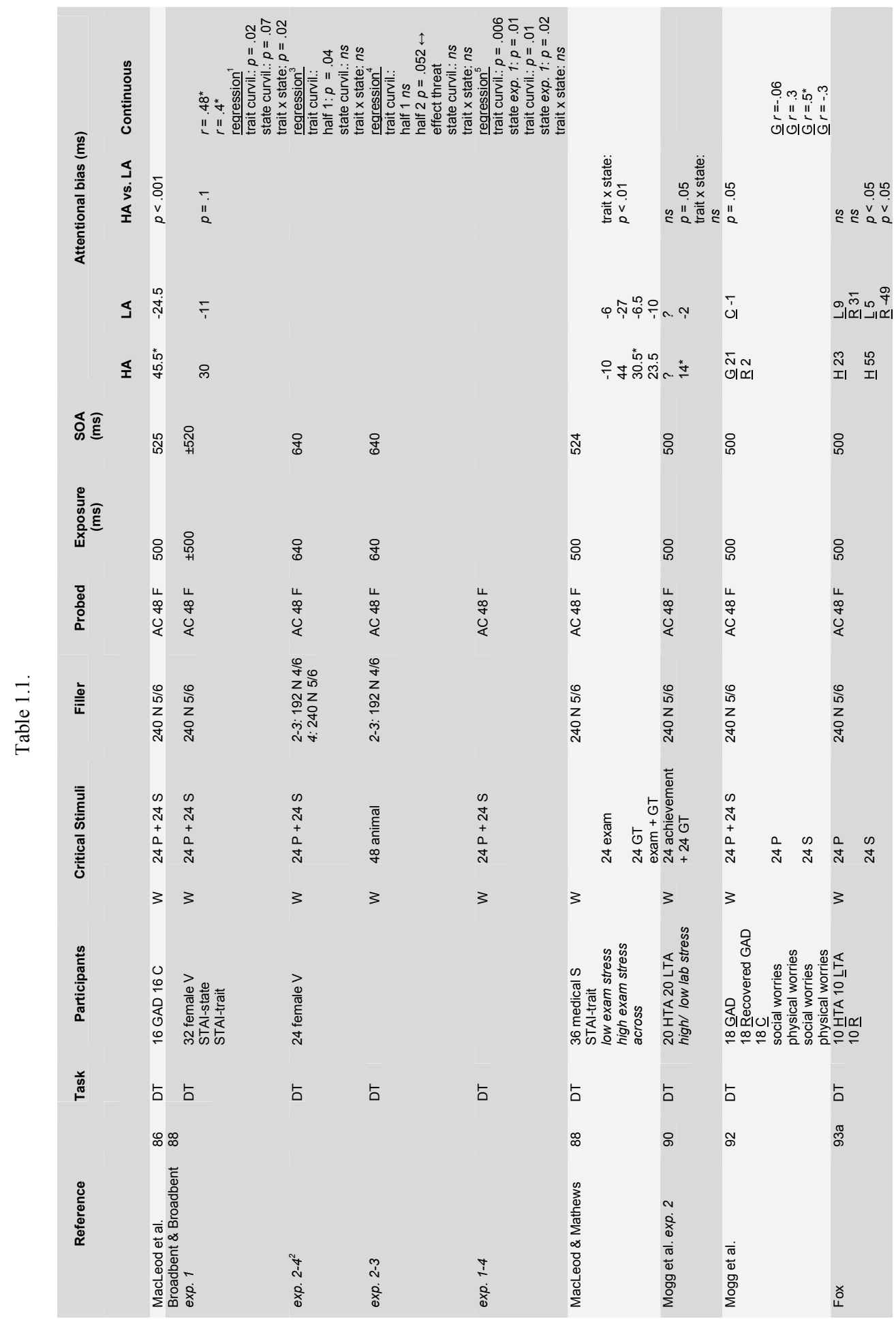


Chapter 1

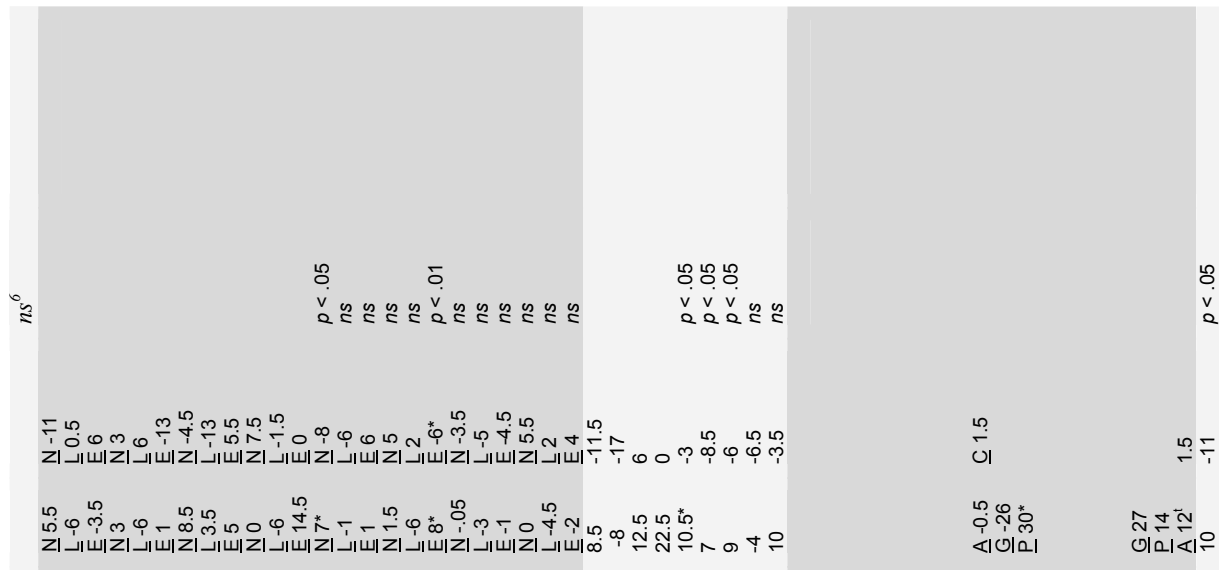

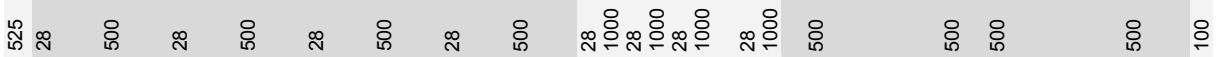

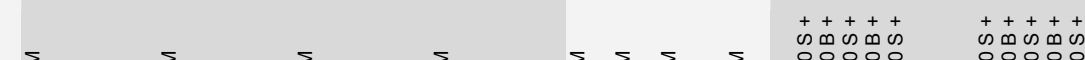

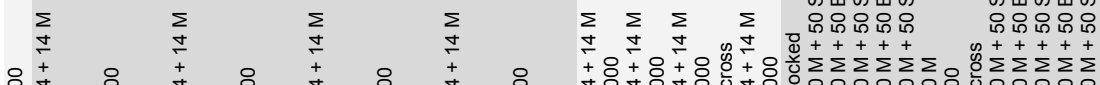

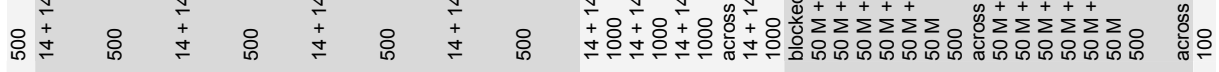

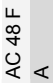

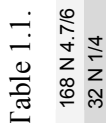

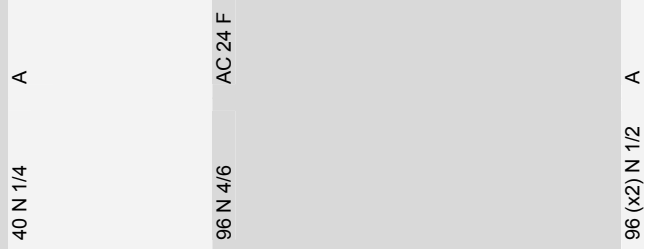

$\stackrel{2}{a}$

i

$\begin{array}{ll}\text { a } & \text { n } \\ 0 & 0\end{array}$

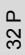

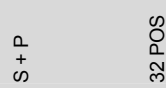

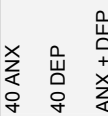

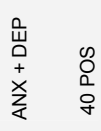

a

$\frac{a}{2}$

33

3

3

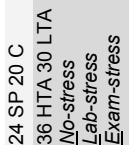

0
0
0
0
0
0

呈

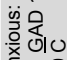

이잉

다돈

임

5

a

เீ

ค

0

ठ ठ

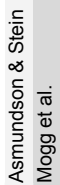

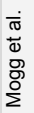



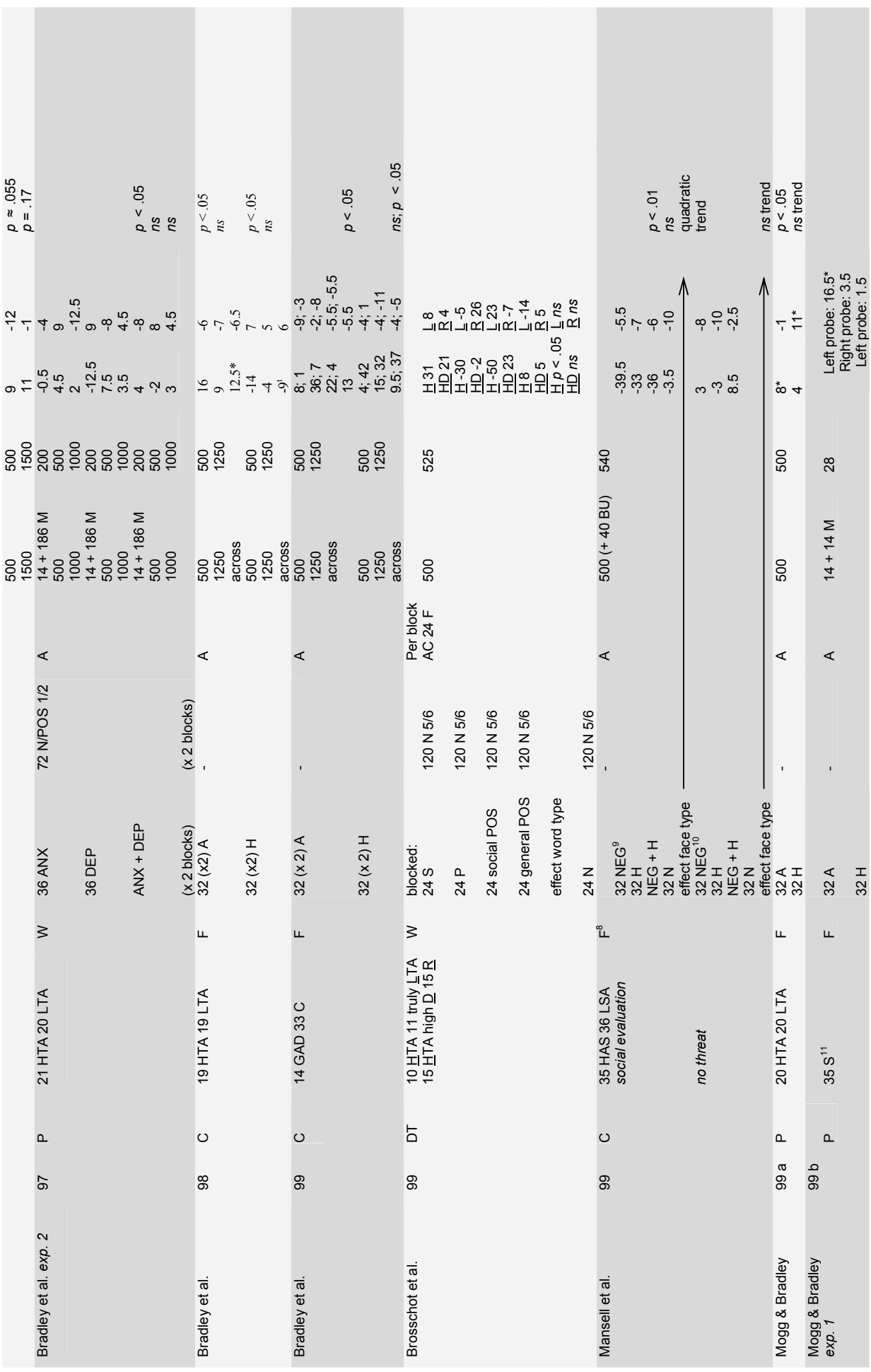


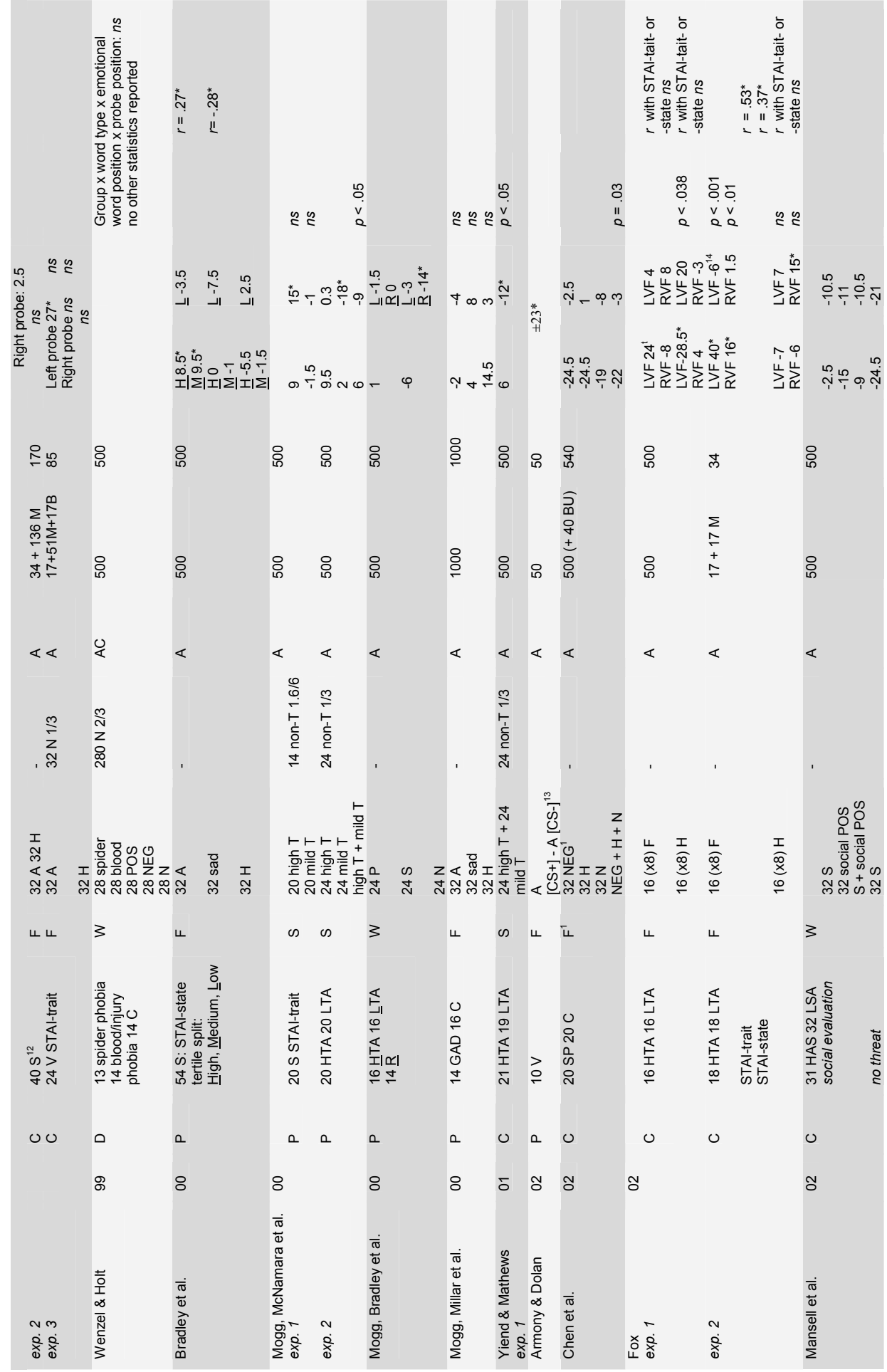




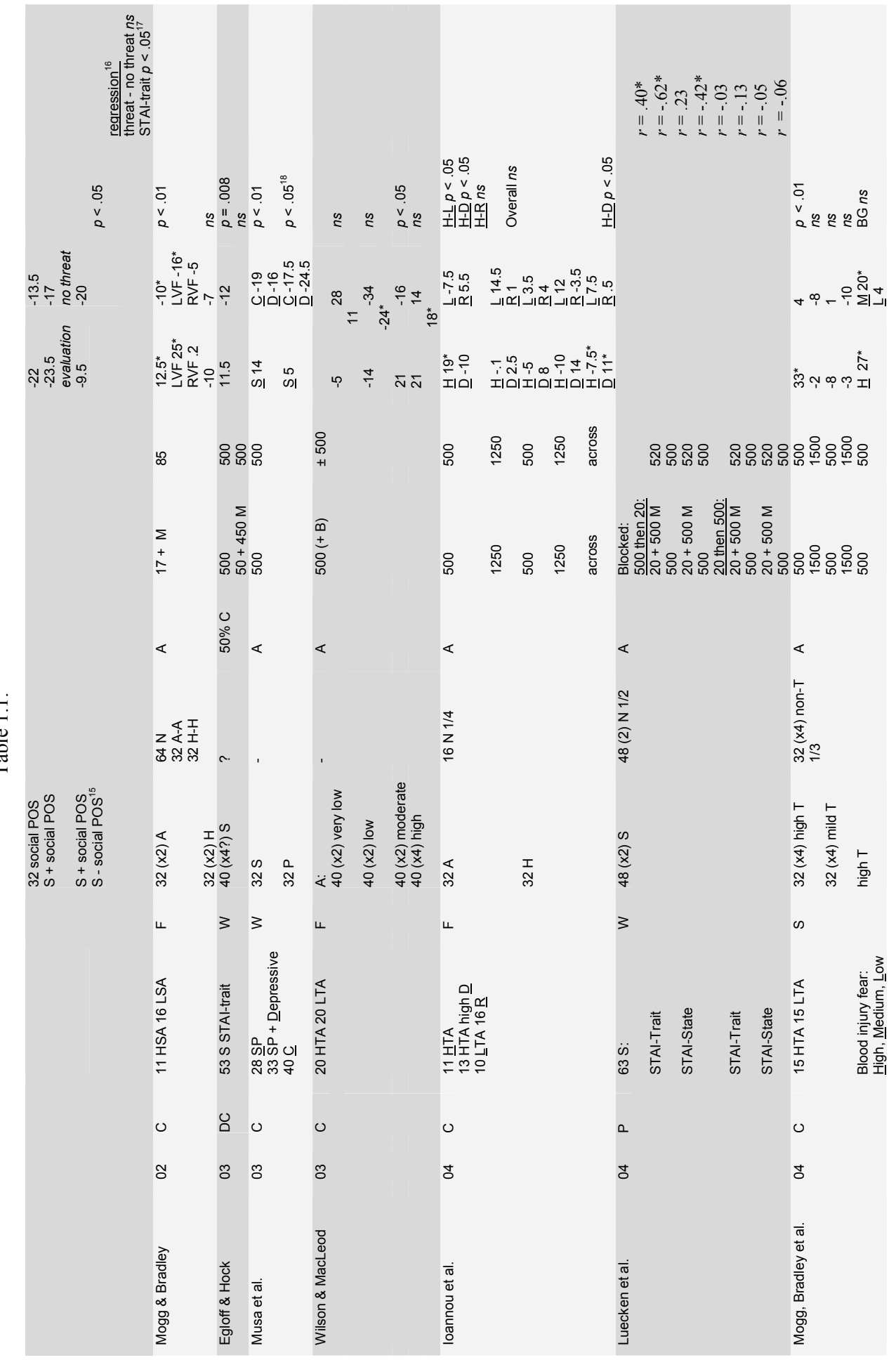


Chapter 1

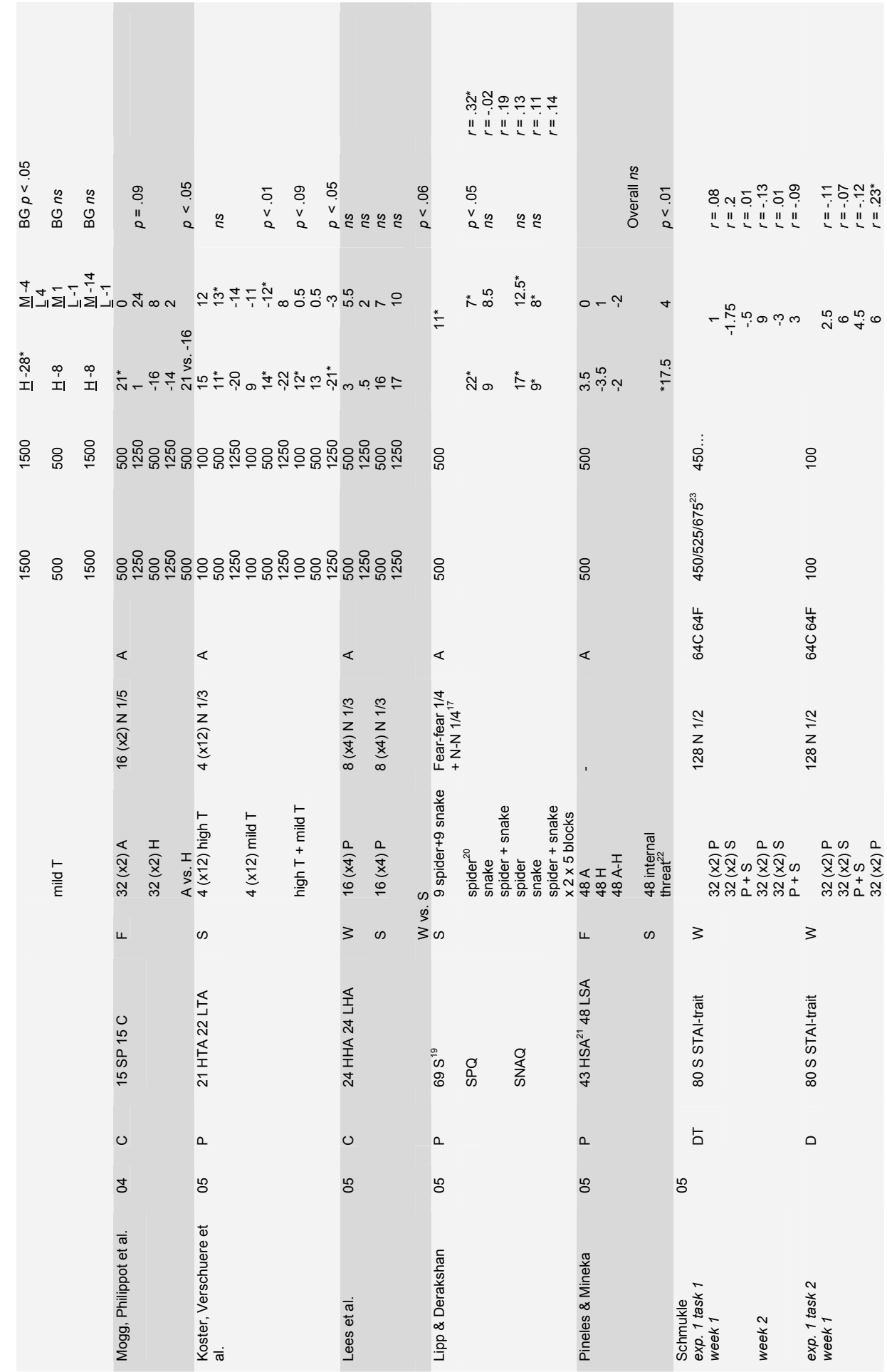




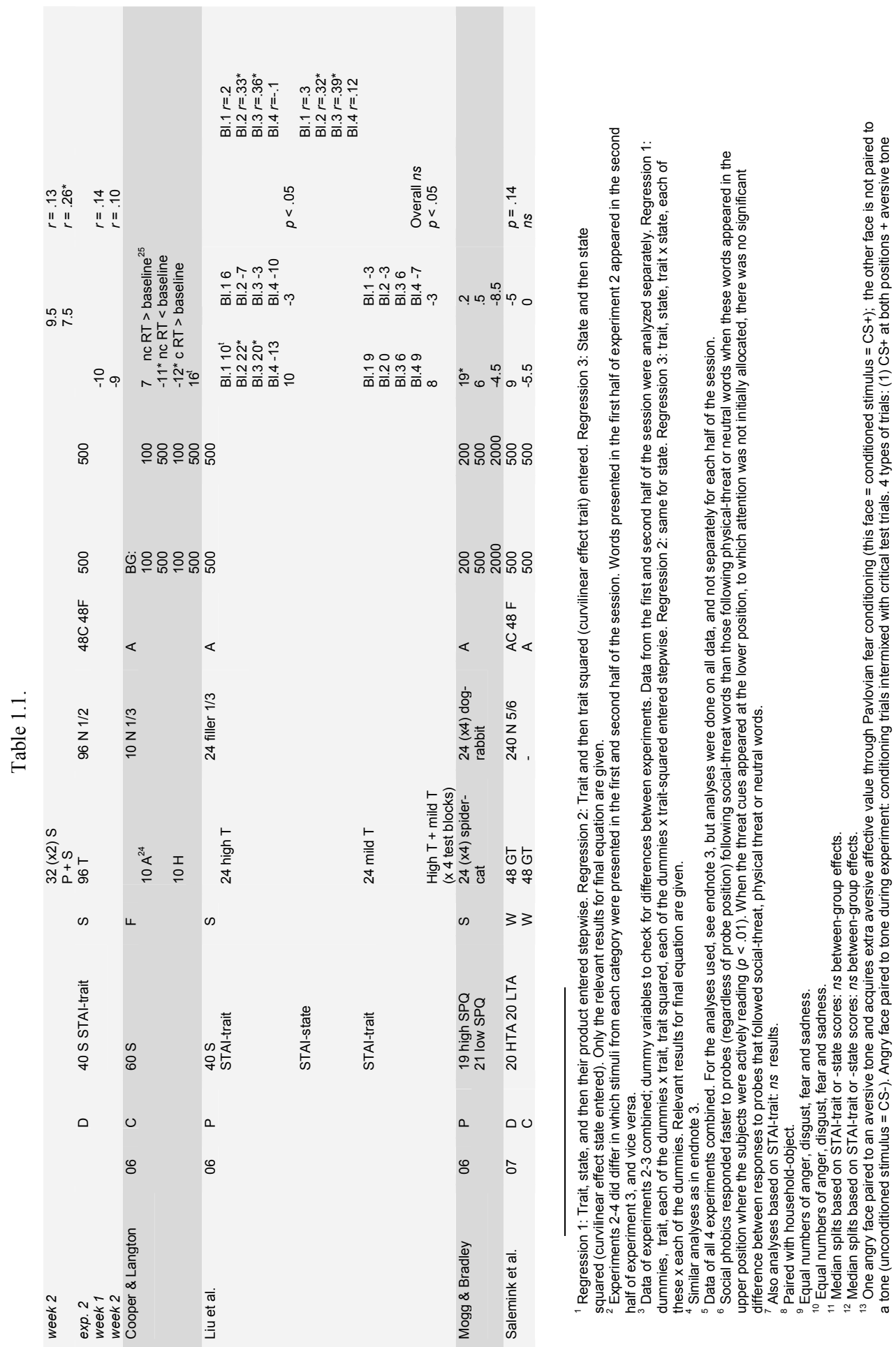


Chapter 1

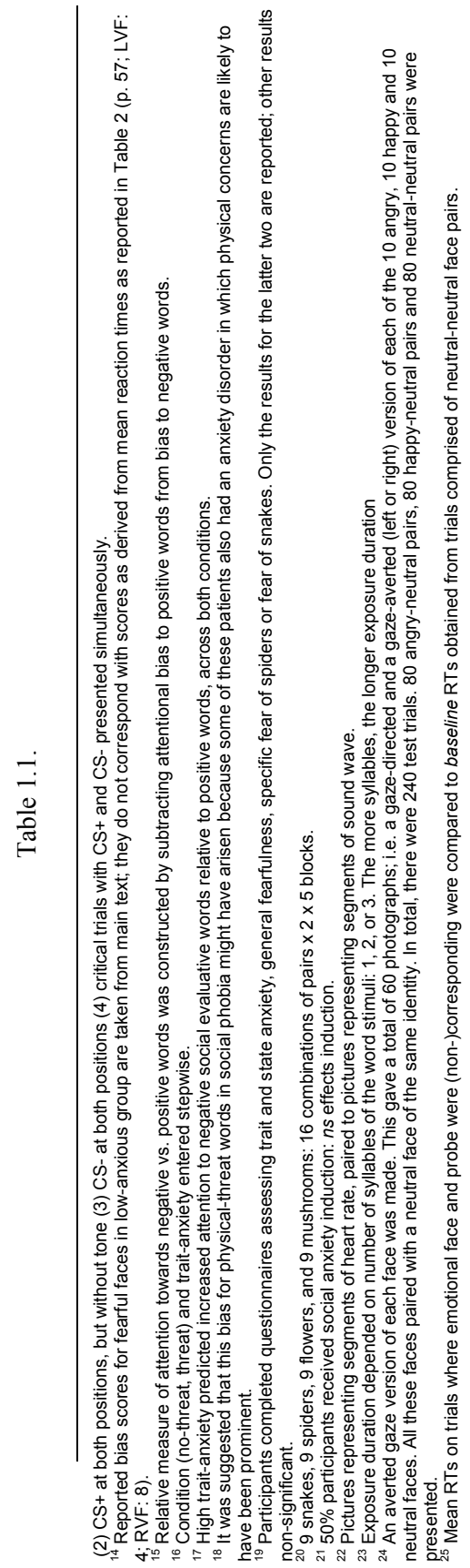


In the following sections, first, the general evidence is considered from dotprobe studies presenting words or pictures for $500 \mathrm{~ms}$, the exposure duration typically employed in dot-probe research. Next, modifications of exposure duration and response option are discussed.

\subsubsection{Dot-probe Effects with 500-ms Stimulus Exposure}

In dot-probe research, it has been common to present stimulus pairs for $500 \mathrm{~ms}$. In general, studies employing this exposure duration have revealed a positive attentional bias score for threat-relevant words in anxious compared to non-anxious individuals, both for anxiety patients (e.g., GAD: MacLeod et al., 1986; Mogg, Mathews, \& Eysenck, 1992; Mathews, Ridgeway, \& Williamson, 1996; panic disorder: Mathews et al., 1996; social phobia: Asmundson \& Stein, 1994; Musa, Lépine, Clark, Mansell, \& Ehlers, 2003; but not in spider or blood/injury phobia: Wenzel \& Holt, 1999) and for people with a high-anxious personality trait (Broadbent \& Broadbent, 1988; MacLeod \& Mathews, 1988; Fox, 1993; Mansell, Ehlers, Clark, \& Chen, 2002), when under stress (MacLeod \& Mathews, 1988; Mogg et al., 1990; Mogg, Bradley, \& Hallowell, 1994). In contrast, truly low-anxious individuals showed no bias for threat-related words and individuals reporting low levels of trait-anxiety combined with high social desirability (called 'repressors') show a negative bias (Fox, 1993; Mogg, Bradley, et al., 2000). In general, no bias has been reported for positive words in high-anxious groups, suggesting that the observed bias effects are not due to some general emotionality effect.

So, even with such abstract stimulus material as words, significant attentional bias effects have been observed. However, words are not very ecologically valid and their threat intensity level is rather weak. Threat-related pictures, like photographs of angry or fearful faces, may have a more intense threat value than words, and they may also be more valid from an evolutionary perspective (Öhman, 1994). It has been shown that pictures of facial expressions are ecologically valid and can depict abstract situations (e.g., interpersonal dominance-submission conflicts; Ekman \& Friesen, 1976). However, a disadvantage of the use of pictures in dot-probe tasks is that it is more difficult to match two pictures in terms of, for example, their physical features (e.g., brightness, color, contrast, number of details) or frequency, than to match two words. For an unambiguous interpretation of the observed bias effects, it is of course highly important that the two members of the stimulus pair do only (systematically) differ in content and not also in some other, irrelevant, aspects.

Dot-probe studies presenting pictorial stimuli, instead of words, for about 500 $\mathrm{ms}$ have revealed a positive attentional bias score for threat-related pictures in anxious compared with non-anxious individuals, both for anxiety patients (faces: GAD: Bradley, Mogg, White, Groom, \& de Bono, 1999; social phobia: Mogg, Philippot, \& Bradley, 2004) and for people with a high-anxious personality trait 


\section{Chapter 1}

(faces: Bradley, Mogg, Falla, \& Hamilton, 1998; Mogg \& Bradley, 1999a; Fox, 2002; low defensiveness: Ioannou, Mogg, \& Bradley, 2004; scenes: Yiend \& Mathews, 2001; Mogg, Bradley, Miles, \& Dixon, 2004; Koster, Verschuere, Crombez, \& Van Damme, 2005; Liu, Qian, Zhou, \& Wang, 2006), when under stress (faces: Bradley, Mogg, \& Millar, 2000). In the studies relying on facial stimuli mentioned above, threat-related faces have been paired to pictures of neutral facial expressions. Studies during which emotional faces have been presented simultaneously with pictures of household objects rather than neutral faces revealed an attentional bias away from emotional (positive and negative) faces, towards objects, in patients with social phobia and in socially anxious students when under social evaluative stress (Chen, Ehlers, Clark, \& Mansell, 2002; Mansell, Clark, Ehlers, \& Chen, 1999). Furthermore, positive attentional bias scores have been obtained in an unselected sample for pictures of spiders and snakes, paired to flowers or mushrooms (Lipp \& Derakshan, 2005). Positive attentional bias scores have also been reported for low-anxious people, but only when confronted with highly threatening pictures (Mogg, McNamara et al., 2000; Wilson \& MacLeod, 2003). In general, dot-probe studies with pictorial stimulus material did not reveal attentional bias effects for positive stimuli in high-anxious individuals, suggesting that the reported bias is not due to a general emotionality effect. Bradley and colleagues (1999) reported a positive attentional bias for happy faces in generalized anxiety disorder, but only in the second half of the task. They speculated that this might be due to mood regulating or repairing strategies that come into play after some time.

Taken together, dot-probe studies presenting words or pictures for about 500 $\mathrm{ms}$ have revealed content-specific attentional bias effects favoring threat-relevant stimuli, both in clinical anxiety and sub-clinical anxiety, and also in low-anxious individuals, but in the latter only for stimuli with high levels of threat intensity. As already mentioned, in previously published dot-probe experiments exposure duration of the stimulus pair and also response options have been manipulated. In the following sections, these manipulations are discussed successively, with both discussions leading to research questions addressed in the presented dissertation work.

\subsubsection{Interpretation \& Time-Course Dot-Probe Effects}

As can be seen in Table 1.1., in more recent dot-probe studies, exposure duration of the stimulus pair has been varied between 14 and 2000 ms. Extremely short exposure durations have been used to investigate whether bias effects also occur for stimuli presented under conditions of restricted awareness. This line of research is discussed in more detail in Section 1.2.3.1., where the focus is on the supposed automaticity of preferential processing in anxiety. In another series of experiments, exposure duration has been varied in order to investigate the time-course of the dot- 
probe effects. Before moving on to a discussion of these studies, a few remarks are in order concerning the interpretation of attentional bias scores.

\subsection{Sub-Components of Attentional Orienting}

The original interpretation of positive attentional bias scores (i.e., relatively fast responses when emotional stimulus and probe spatially correspond) is that they reflect a tendency to preferentially shift (move, allocate, deploy) attention towards the (spatial location of the) emotional stimulus, whereas negative values have been taken to indicate an attentional shift away from the emotional stimulus, towards the neutral one. ${ }^{4}$ However, as explained below, alternative interpretations are possible.

In basic cognitive research, it has become generally accepted that visual orienting can be broken down into several sub-operations. For example, when an individual is attending to an object, attention is engaged at the location of this object. If attention is to be allocated elsewhere, it must first be disengaged from its current focus and then be shifted to the alternative location (e.g., Posner \& Petersen, 1990). Inspired by these ideas, the question has been raised as to how these three attentional operations - engagement, shifting and disengagement - are biased in anxiety (e.g., Mogg, Bradley, de Bono, \& Painter, 1997). Dot-probe results for stimuli presented for 14 or $500 \mathrm{~ms}$ have been taken as evidence for an anxiety-related bias in initial orienting and early attentional engagement (e.g., Mogg et al., 1997; Bradley et al., 1998). However, this interpretation has been debated for bias effects elicited with 500-ms exposure (e.g., Fox et al., 2001; Koster, Crombez, Verschuere, $\&$ De Houwer, 2004). Fox (e.g., 2004; Fox et al., 2001) recently pointed out that when stimulus pairs are presented for $500 \mathrm{~ms}$, it is actually possible that participants switch covert attention several times between the two stimulus locations. Covert attentional orienting refers to the allocating of attention to a point in space in the absence of movements of the eyes, head or whole body. Fox argued that switching covert attention between the two stimulus locations is an obvious strategy for participants to adopt during a dot-probe task, for both locations are task relevant (remember that the probe occurs with an equal probability at either location). The question can be raised whether threat-related stimuli affect early shifts of attention and attract attention to themselves in the first place. Or is it the case that once threat-related stimuli are detected, it is difficult to disengage attention from them? In dot-probe tasks, differences in response latencies between corresponding and

\footnotetext{
${ }^{4}$ The spatial aspect of the bias effects might be the mere outcome of task construct. During the task, stimuli differing in emotional relevance are presented at separate spatial locations. Whereas it seems to be logical to assume that fear for a specific object is characterized by spatial attentional processes, it is less plausible to assume that attentional processes involved in more generalized anxiety or worrying are inherently spatial in nature.
} 
non-corresponding trials may result from biases in either shifting or disengagement of attention, or a mixture of both. In other words, positive attentional bias scores may arise from a preferential shift of attention towards the emotional stimulus, but also from difficulty in disengaging attention from the (location of the) emotional stimulus (e.g., Fox et al., 2001; Fox, 2004; Koster, Crombez, Verschuere, et al., 2004). Researchers assuming that dot-probe bias effects assessed with $500 \mathrm{~ms}$ exposure reflect initial orienting (Bradley et al., 1998; Mogg, Bradley, et al., 2004; Mogg, Philippot, et al., 2004; Lees, Mogg, \& Bradley, 2005) refer to Bradley et al. (2000)'s finding of concordance between the bias derived from dot-probe reaction time data (with $500 \mathrm{~ms}$ exposure) and the direction of initial shift in overt gaze to emotional faces. ${ }^{5}$

In order to study the separate components of biased attentional orienting for emotional stimuli more directly, modified versions of Posner (1980)'s exogenous spatial cueing task have been developed (e.g., Stormark, Nordby, \& Hugdahl, 1995; Fox et al., 2001; Yiend \& Mathews, 2001). In the original version of the exogenous cueing task, a cue is presented at one of two locations (e.g. abrupt onset of light). After a delay, a probe is presented, equally likely at the location of the preceding cue or at the other location. During baseline trials, a neutral cue is presented, providing no spatial information (e.g., abrupt onset cue at both locations). It has consistently been found that, at least when the interval between cue and probe onset is shorter than about $150 \mathrm{~ms}$, responses are faster to probes occurring at the cued location (valid) than to probes at baseline trials, but that responses to probes occurring at the other location than the cue (invalid) are slower (for an overview, see for example Rafal \& Henik, 1994; Yantis, 1996). ${ }^{6}$ The idea is that a peripheral cue rapidly captures attention, resulting in facilitated detection of probes replacing this cue. However, when a probe appears at another location than the preceding cue, attention must first be disengaged from the attended cue location, shifted towards and engaged at the probe location, resulting in slower detection of these probes.

In the exogenous cueing task designed to study biased attentional orienting for emotional information, the peripheral cues have either an emotional or a neutral

\footnotetext{
${ }^{5}$ In most dot-probe studies, in order to minimize eye movements, participants have just been asked to try and keep their eyes focused on a central fixation cross. Still, in these studies, eye movements have not been registered. Mathews et al. (1996), to ensure that their participants were fixating a point midway between the members of the stimulus pair, asked them to read a digit number centrally placed between the two stimuli (see also Egloff \& Hock, 2003). In the first series of dot-probe tasks, participants had to read aloud the top word of the stimulus pair (see Table 1.1). This extra instruction complicates the interpretation of the dot-probe effects (see main text Section 1.2.2.3.). When eye movements are not measured, it is of course unclear in what way and to what extent the dot-probe effects depend on overt vs. covert orienting.

${ }^{6}$ At longer intervals (i.e., in excess of about $300 \mathrm{~ms}$ ), responses are typically slower at valid locations (inhibition of return; Posner \& Cohen, 1984).
} 
content (e.g., angry, happy, and neutral facial expressions). ${ }^{7}$ In several studies applying this task, it has been found that responses of anxious individuals are especially slow when a threat-related stimulus is followed by a probe at the other location, compared to when a positive or neutral stimulus is presented. This has been found for people reporting high levels of state-anxiety (faces: Fox et al., 20018), traitanxiety (faces: Fox et al., 2002; Georgiou et al., 2005; scenes: Yiend \& Mathews, 2001 exp.2; Koster, Crombez, Verschuere, Van Damme, \& Wiersema, 2006; arrows predicting the loss of points: Derryberry \& Reed, 2002; but see Broomfield \& Turpin, 2005: rapid disengagement from threat words in high-trait-anxious), social phobics (social-threat words: Amir, Elias, Klumpp, \& Przeworski, 2003), and in the general population for classically conditioned fear stimuli (Koster, Crombez, Van Damme, Verschuere, \& De Houwer, 2005; Van Damme et al., 2006). Furthermore, it has been found that especially in high-trait-anxious (female) participants, inhibition-of-return effects (IOR; i.e. slower responses on valid trials with cue-probe intervals longer than about 300 ms; e.g., Posner \& Cohen, 1984) were reduced following threat cues, relative to positive or neutral cues (faces: Fox et al., 2002; scenes: Waters, Nitz, Craske, \& Johnson, 2007). The slow-down on invalid trials and the reduction of IOR effects after threat cues have been taken as evidence for difficult disengagement associated with anxiety.

In most of the aforementioned studies, no effects of valence were observed at valid trials. Fox et al. (2001, p. 697) argued that it might be too much to expect that the threat value of the cue would further speed the orienting of attention on valid trials and that this modified exogenous cueing task is not a fair test of the shift component of attention. However, in recent studies it has been found that, on valid trials, responses are even faster when a fear-conditioned stimulus (in unselected sample: Koster, Crombez, et al., 2005; Van Damme et al., 2006) or a highly threatening picture (in high-trait-anxious: Koster et al., 2006) is presented as cue, than when a neutral stimulus appeared.

Taken together, the data from the modified version of the exogenous cueing task seem to suggest that anxiety is characterized by biases in attentional shifting to or engagement with as well as in disengagement from threat. Note that this task is

\footnotetext{
${ }^{7}$ Whereas in a typical exogenous cueing task the cue is not predictive for probe location (i.e., $50 \%$ valid, $50 \%$ invalid), in most affective versions of this task, there has been a higher proportion of valid than invalid trials (Stormark, et al., 1995; Fox et al., 2001; Fox, Russo, \& Dutton, 2002 exp. 1; Amir et al., 2003; Koster, Crombez, et al., 2005; Broomfield \& Turpin, 2005; Van Damme, Crombez, Hermans, Koster, \& Eccleston, 2006). So, in these latter studies, the peripheral cues predicted the likely probe location. It is possible that such cues reflect the conjoint action of both exogenous orienting (reflexive orienting triggered by salient peripheral stimuli) and endogenous orienting (influenced by subjects' expectations) (see e.g., Rafal \& Henik, 1994).

${ }^{8}$ Fox and colleagues (2001) found also delayed disengagement effects for threat-related, relative to positive or neutral words in the whole sample. However, there was no difference between high- and low-anxious groups for this effect.
} 
highly similar to a typical dot-probe task, except that not an emotional-neutral stimulus pair is presented, but just a single emotional or neutral stimulus. So, in contrast to the dot-probe task, this single-cue cueing task does not measure prioritized attentional processing of emotionally relevant information over simultaneously presented neutral information.

In an attempt to figure out which attentional mechanisms underlie typical dotprobe effects, Koster, Crombez, Verschuere, et al. (2004) took in their analyses of dot-probe results also response latencies on neutral trials into account. In virtually all previous dot-probe studies, besides critical emotional-neutral stimulus pairs, pairs consisting of two neutral stimuli have also been presented. These neutral pairs did only serve as filler material and were excluded from further statistical analyses. Koster and colleagues argued that biases in attentional shifting (engagement) in favor of emotional stimuli ('vigilance') should lead to faster responses on emotionalneutral trials during which the emotional stimulus and the probe were spatially corresponding (congruent trials) compared to neutral trials. Delayed disengagement from emotional stimuli should lead to slower responses on emotional-neutral trials during which the emotional stimulus and the probe did not spatially correspond (incongruent trials) compared to neutral trials. Koster et al. observed slower responses on incongruent than on neutral trials for pictures depicting mildly or highly threatening scenes (500 ms exposure) in a normal sample; there were no significant differences in response latency between congruent and neutral trials. Following the same approach as Koster et al., Salemink et al. (2006) observed differences between high- and low-trait anxious individuals on disengagement from, but not on shifting towards threat words (500 ms exposure). That is, their high-trait anxious participants were especially slow on incongruent trials compared to neutral trials when a threat-related word was presented. These response patterns have been taken to imply that positive attentional bias scores do not unambiguously reflect a preferential shift of attention towards threat, but that they may also reflect delayed disengagement from threat.

\subsection{Time-Course of Anxiety-Related Attentional Bias}

As mentioned before, in a whole series of dot-probe studies, exposure duration of the stimulus pair has been systematically varied in order to study the dynamic timecourse of anxiety-related attentional bias (see Table 1.1.). To investigate whether anxious individuals, after initial orienting direct their attention away (avoidance) or whether they maintain their attention on the source of threat (difficult disengagement), dot-probe studies have been conducted in which the exposure duration of the stimulus pair has been manipulated systematically. The precise course and timescale of attentional bias effects seem to depend on the threat value of the stimuli, the anxiety group under consideration (clinical vs. non-clinical; state vs. trait anxiety; 
general anxiety vs. specific fear), and specific factors in the paradigm (see Koster, Verschuere, et al., 2005). To date, two dot-probe studies provided some evidence for avoidance following initial orienting: away from high-threat scenes in individuals reporting high levels of blood-injury fear (Mogg, Bradley, et al., 2004), and away from threat pictures in high-trait anxious individuals (Koster, Verschuere, et al., 2005). Other dot-probe studies have reported for long presentation durations nonsignificant trends for a bias towards threat-related words or angry faces in nonclinical anxiety (Mogg et al., 1997; Bradley et al., 1998) or no significant bias effects at all, for high-threat scenes or threat-related words in high-trait anxiety (Mogg, Bradley, et al., 2004; Bradley, Mogg, \& Lee, 1997 exp. 2), for angry faces in social phobia (Mogg, Philippot, et al., 2004), for health-threat pictures in health anxiety (Lees et al., 2005) or for spider pictures in spider fear (Mogg \& Bradley, 2006). It has been suggested that with longer exposure durations, attention may shift repeatedly between the members of the stimulus pair. These approach and avoidance tendencies may counteract each other resulting in no significant anxiety-related bias effects (e.g., Mogg, Philippot, et al., 2004).

As can be seen in Table 1.1., in virtually all dot-probe studies that manipulated exposure duration of the stimulus pair, probes appeared immediately following the stimulus pair (or mask). That is, longer exposure durations were typically paired with larger intervals between the onset of the stimulus pair and the onset of the probe (stimulus-probe onset asynchrony; SOA). In dot-probe tasks, biased attention is 'tapped' in its time-course at the moment the probe is presented. Thus the larger the SOA, the later the bias is tapped in its time-course. On the other hand, with longer exposure durations, there is more time to process stimulus content. The study presented in Chapter 7 is a first attempt to disentangle the effects of exposure duration (processing time) and SOA (moment at which bias is tapped).

To recap, to investigate the dynamic time-course of attentional bias effects, researchers have systematically manipulated the exposure duration of the stimulus pairs in dot-probe tasks. In doing this, a snapshot has been taken of attentional bias effects at different moments in time. Other ways that have been applied to map the temporal course of attentional bias effects are measuring the direction and latency of eye movements to emotional stimuli (e.g., Hermans, Vansteenwegen, \& Eelen, 1991; Rohner, 2002; Bradley et al., 2000; Mogg, Millar, \& Bradley, 2000; Garner, Mogg, \& Bradley, 2006) or recording event-related brain potentials from the scalp (e.g., Eimer \& Kiss, 2007). A detailed discussion of these alternative measures is beyond the scope of this introduction.

In Chapter 6, an alternative way of analyzing dot-probe reaction time data is introduced, that, according to us, can be used to assess the temporal dynamics of attentional bias effects. More specifically, it is argued that distributional analyses of responses to the probe might inform us about the time-course of the hypothesized effects. 


\subsubsection{Methodological Considerations on Dot-Probe Tasks}

Three basic types of the dot-probe paradigm can be distinguished based on the response to be given to the probe. In probe-detection tasks, participants press a button on a response box as soon as the probe appears (e.g., MacLeod et al., 1986). In probe-position tasks (e.g., Mogg et al., 1994), participants decide during every trial, by pressing one of two buttons, in which of two positions the probe occurred. In probe-classification or -differentiation tasks (e.g., Bradley et al., 1998), participants indicate which type of probe was presented. Probe-detection, probe-position as well as probe-classification studies seem to provide evidence consistent with anxiety-related attentional bias favoring threat-relevant stimuli. However, it is important to note here that these tasks differ in their relative merits. When taking a closer look at the precise methodology adopted in these different versions, some drawbacks can be identified that may complicate the interpretation of the obtained results. For a methodological discussion, the reader is referred to, for example, Mogg and Bradley (1999a). For a direct comparison of results from a probe-classification and a probe-position or -detection study, see Mogg and Bradley (1999a), and Salemink et al. (2007), respectively.

As can be seen in Table 1.1., in the first series of dot-probe studies in anxiety, participants had to detect the probe as quickly and accurately as possible. In these studies, the probe did not appear at every trial ( $5 / 6$ of all trials were fillers), but all critical word pairs were followed by a probe. There was a contingent relationship between the occurrence of a threat word and the occurrence of the probe (cf. Mogg et al., 1994). So, threat words predicted that a probe would be presented. Participants may differ in the extent to which they detect this relationship. Because any effect of expectancy would occur on all trials during which a threat word was presented, irrespective of the spatial correspondence of threat word and probe, such effect would not be confounded with spatial attentional bias. In these early probedetection studies, participants also had to read aloud the word at the upper position during each trial. So, in fact, participants in these studies were instructed to attend the upper position at the initiation of each trial. In this situation, if the probe appeared at the upper position, bias scores might reflect a delay in attentional disengagement from this location. However, if the probe appeared at the lower position, bias scores might reflect a bias in attentional shifting (Asmundson \& Stein, 1994; Chen et al., 2002; Fox et al., 2001; Wells \& Matthews, 1994).

To remove the confound between the probability of occurrence of threat word and probe, Mogg et al. (1994) presented a probe during every trial and asked their participants to indicate its position by pressing the spatially corresponding key on a response box (i.e., if probe in upper position, press upper key; if probe in bottom position, press lower key). The advantages of obtaining data from all trials are obvious. First, the task can be made shorter, something that is especially advanta- 
geous when testing individuals that have difficulty sustaining attention over a long monotonous task (Mogg \& Bradley, 1999a). In addition, more experimental manipulations can be included, without the task becoming too long. Furthermore, because of the direct spatial correspondence of probe and response (e.g., both upper position or both lower position) probe-position tasks are very easy to carry out and may therefore seem particularly suitable for testing, for example, clinical samples (Mogg \& Bradley, 1999a). Unfortunately, while performing a probe position task, participants may adopt the strategy to focus their attention on just one of the two possible probe positions and base their response on the presence or absence of the probe on that location, making the task less sensitive to attentional biases (Bradley et al., 1998).

In previous dot-probe literature, the direct correspondence of probe and response in probe-position tasks has been considered a positive aspect of the task, making the task easy to perform (Mogg \& Bradley, 1999a). However, because of this systematic correspondence of probe and correct response, response bias effects could contribute to the attentional bias scores derived from probe-position tasks. Each time probe and emotional stimulus appear in the same position, the correct response is also spatially corresponding with the emotional stimulus; each time probe and emotional stimulus appear in different positions, the correct response does also not correspond with the emotional stimulus. Therefore, attentional bias scores (mean reaction time when emotional stimulus and probe are noncorresponding minus mean reaction time when emotional stimulus and probe are corresponding) are $100 \%$ confounded with any response bias. This is illustrated in Table 6.1. (Chapter 6). Note that in probe-detection tasks and previously published probe-classification tasks, there is no systematic spatial correspondence of emotional stimulus, probe, and response. In probe-detection tasks, there is only one key that has to be pressed. In probe-classification tasks, positions of emotional stimulus, probe, and response are typically balanced. Furthermore, in previous probeclassification tasks spatial correspondence of emotional stimulus and response has been avoided (e.g. stimulus positions vertically oriented (top, bottom), but response positions horizontally oriented (left, right)). The issue of response bias as a confounding factor in the interpretation of attentional bias effects in probe-position tasks has been overlooked by attentional bias researchers (but see Fox et al., 2001, making a similar remark for modified exogenous cueing tasks). This idea is further elaborated in Chapter 6. We argue that such response bias is interesting in itself for research on preferential processing of emotional information (see also Section 1.3.3.2.).

In probe-classification tasks, it is impossible to select the correct response when attention is focused on only one of the alternative probe positions. Furthermore, in these tasks, the mapping between probe and response is more arbitrary and may be more difficult to learn than in probe-position tasks. Of course, task difficulty de- 


\section{Chapter 1}

pends largely on the discrimination to be made (e.g. left key to ":", right key to ".." vs. left key to " $\longleftarrow$ ", right key to “ $\rightarrow$ "). The more difficult the task, the slower the responses, the more errors made (less trials left for statistical analysis) and the more variance in reaction times. This may influence the sensitivity of the task in measuring attentional bias effects (Mogg \& Bradley, 1998).

Finally, as can be seen in Table 1.1., in several probe-position and especially probe-classification studies, no neutral-neutral filler pairs have been presented, whereas in probe-detection studies a certain proportion of the trials, often the majority, are fillers. Of course, when an emotional stimulus appears on every trial, the focus of the experiment on emotional processing may become more transparent to participants than when only occasionally an emotional stimulus is presented. Furthermore, the build-up of expectancy, arousal, priming, awareness of emotional stimulus content etc. across trials may differ between studies including different proportions of neutral filler material.

Taken together, previous dot-probe tasks differ in several task parameters, like response position, neutral filler material, and whether or not the stimulus on the top position has to be read aloud. Although these methodological differences may seem to be of little importance at first sight, they may affect the observed bias effects in a significant way. Such differences in exact methodology may explain some of the mixed findings that various studies report.

\subsubsection{Automatic Preferential Processing?}

The reader may remember from Section 1.1.2. that cognitive models on anxiety do not only highlight the role of preferential processing of threat, but also emphasize automatic processing of threat (e.g., Williams et al., 1988; Eysenck, 1992; Öhman, 1994).

A wide variety of definitions and views on automaticity and its relationship with selective attention has been formulated (Shiffrin \& Schneider, 1977; Bargh, 1992; Logan, 1992; Treisman, Vieira, \& Hayes, 1992; LaBerge, 2002). Originally, automatic processes have been defined as being involuntary, unintentional, autonomous, effortless and operating outside conscious of awareness. In contrast, controlled processes have been described as being intentional, voluntary, flexible, resource or capacity limited, requiring extra efforts, and partially accessible to awareness. According to a strong definition of automaticity, automatic and controlled processes meet all these criteria. However, this strong definition and rigid dichotomy between pure automatic and controlled processes has been criticized, in part because there are instances in which some, but not all of these characteristics apply (Bargh, 1992; Cohen, Servan-Schreiber \& McClelland, 1992). Bargh (1992) drew a distinction between pre- and post-conscious automatic processes. For the occurrence of pre- 
conscious processes, only the recent presence of a relevant stimulus is necessary. For post-conscious processes to occur, conscious attentional processing concerning this relevant stimulus is also required (e.g., priming).

A substantial amount of behavioral and neuroscientific research has been dedicated to the automatic processing of emotional stimuli (for a review, see for example Compton, 2003). The following discussion is restricted to behavioral evidence for automatic processing in anxiety. The nature of automatic processing in anxiety follows directly from the definition of automaticity above (McNally, 1995; Wells \& Matthews, 1994). In what sense is anxiety-related processing of threat automatic?

To start with, the question can be raised in what sense the mechanisms underlying emotional Stroop and dot-probe effects as described in the previous sections are automatic. It is logical to assume that the processes underlying emotional Stroop effects are automatic in the sense that they occur involuntarily. However, these processes are unlikely to be automatic in the sense that they are capacity-free. For if they would be completely capacity-free and not depend on extra cognitive resources to evolve, then interference with color-naming would not occur at all (McNally, 1995). Remember that the original interpretation of emotional Stroop effects is that emotional word content automatically captures attention (e.g. Pratto \& John, 1991), suggesting that processing of threat-related stimuli is fast and occurs at an early stage of processing. Recent research, however, suggests that the largest portion of the emotional Stroop effect operates between trials rather than within a single trial (McKenna \& Sharma, 2004; Phaf \& Kan, in press). These findings raise doubt about the immediacy of the emotional Stroop interference.

In dot-probe literature, it has been (tacitly) assumed that in anxiety, a threatrelated stimulus attracts attention reflexively towards its peripheral location, resulting in faster responses to probes occurring at this location. If so, then it is expected that attentional orienting towards threat in dot-probe tasks shows the same characteristics as observed for exogenous orienting towards perceptually salient stimuli. Exogenous attentional orienting is, for example, not affected by memory load or expectations on stimulus position and cannot be inhibited voluntarily (Jonides, 1981). To date, it can however not be excluded that endogenous orienting, based on subject's expectations and plans, is also involved in anxiety-related dot-probe effects. Furthermore, based on findings from modified exogenous cueing data suggesting that individuals with good attentional control were better able to shift attention away from threat locations (Derryberry \& Reed, 2002), it can be expected that attentional processing in anxiety as measured with the dot-probe task is not fully automatic and may be subjective to top-down attentional control.

Recently, an affective version of the attentional blink paradigm has been used to investigate whether the processing of threat-related stimuli requires attentional resources (e.g., Fox, Russo, \& Georgiou, 2005). On every trial, a stream of single stimuli was presented, one stimulus after the other, in rapid succession. Attentional 


\section{Chapter 1}

blink refers to impaired performance (i.e., less accurate) in response to a second target stimulus in the series when also a response to an earlier target stimulus is required, within a certain temporal interval, compared to when no response has to be given to this earlier stimulus. This effect is taken to reflect limitations in attentional resources. In short, Fox et al. (2005) have observed attentional blinks when the first target was a neutral stimulus (e.g., flower or mushroom), and the second target a fearful or happy face, suggesting that emotion processing is not fully automatic and needs attentional resources. In high-trait-anxious participants, the attentional blink for fearful faces was reduced relative to the blink for happy faces, supporting the idea that anxiety is characterized by a reduced ability to inhibit the processing of threat. Note that in the attentional blink paradigm as described above, single stimuli are presented successively at the centre of the screen, and therefore, this task does not measure prioritized spatial attentional processing of emotionally relevant information over simultaneously presented neutral information.

Evidence as to the automaticity of preferential processing of threat comes largely from Stroop and dot-probe studies during which stimuli have been presented under conditions of restricted awareness and from visual search experiments. In what follows, these two lines of research are discussed successively.

\subsubsection{Role of Stimulus Awareness}

Much of the debate about the nature of automatic processing in anxiety has hinged on the role of stimulus awareness. Does preferential processing of threat also occur for stimuli that are not consciously perceived? To address this question, modified Stroop and dot-probe tasks have been used in which stimuli were presented outside of awareness or at least under conditions of restricted awareness. Before passing on to a discussion of these studies, some considerations about the dissociation between conscious and unconscious processing are in order.

\subsection{Conscious versus Unconscious Processing}

There is a lot of disagreement about the best approach to distinguish between conscious and unconscious perception / processing (e.g., Holender, 1986; Merikle, Smilek, \& Eastwood, 2001). In order to study the processing of information that is not consciously perceived, stimuli have been presented in a perceptually degraded form. For example, researchers have tried to prevent, or at least to reduce, conscious perception of stimulus content by presenting stimuli for just a few milliseconds, followed by a mask (backward masking; Marcel, 1983; Holender, 1986).

An important distinction has been made between subjective and objective awareness thresholds (Cheesman \& Merikle, 1986; Merikle, 1992; Merikle et al., 2001; Snodgrass, Bernat, \& Shevrin, 2004). The subjective threshold has been set 
on that level of discriminative responding for which subjects claim not to be able to detect (or recognize) the perceptual stimulus, although they perform better than chance. The objective threshold is determined by performance at chance in guessing on a forced-choice task (e.g., lexical decision or presence/absence). One disadvantage of subjective threshold measures is that they may be influenced by individual differences in response criteria. Furthermore, subjective measures may just reflect interpretations of the question asked or some traces of conscious perception. In general, objective measures provide lower threshold values than subjective measures, and may give an underestimation of the apparent influence of information perceived without awareness. As a result, the chance of incorrectly assuming that some processing occurred without awareness is reduced. Such a conservative test is preferred in experimental research, in which one has to be sure that stimulus presentation is below the (reportable) perceptual threshold (e.g., Mogg \& Bradley, 1998). It is important to note here that different objective thresholds may be obtained by using different forced choice tasks (Marcel, 1983). In virtually all previously published subliminal dot-probe and Stroop tasks, objective awareness checks have been presented.

A possibly stronger criterion to distinguish between unconscious and conscious processes than only determining perceptual thresholds is to demonstrate that these processes are also qualitatively different from each other or to show that the presumed unconscious effect is stronger than the conscious effect (Dixon, 1971; Cheesman \& Merikle, 1986; Merikle, 1992; see also Holender, 1986). Cheesman and Merikle, for example, feel that a two-fold approach based on subjective thresholds and qualitative differences is needed to distinguish conscious from unconscious perceptual processes. Some other researchers prefer to completely avoid the notion of an awareness threshold (Phaf $\&$ Kan, in press). They favor the terms "sub- and supra-optimal" above "sub- and supra-liminal".

\subsection{Anxiety-Related Attentional Bias: Role of Awareness}

To investigate whether anxiety-related attentional bias effects occur independent of awareness, as predicted by Williams et al. (1988), Stroop and dot-probe studies were carried out in which stimulus pairs were presented very briefly and masked. See Table 1.1. for an overview of subliminal dot-probe studies.

Even under these conditions of restricted awareness, significant anxiety-related bias effects have been observed, both in sub-clinical and pathological anxiety (e.g., Stroop: MacLeod \& Rutherford, 1992; MacLeod \& Hagan, 1992; Mogg, Bradley, Williams, \& Mathews, 1993; Bradley, Mogg, Millar, \& White, 1995; Van Honk, Tuiten, De Haan, Van den Hout, \& Stam, 2001; Dot-probe with words: Bradley, 


\section{Chapter 1}

Mogg, Lee, 1997; Mogg et al., 1994; Mogg, Bradley, \& Williams, 1995; Dot-probe with faces: Fox, 1996, 2002; Mogg \& Bradley, 1999b, 2002). ${ }^{9}$

The effects for subliminal stimuli were sometimes qualitatively different (MacLeod \& Rutherford, 1992; Mogg et al., 1994) and stronger (Fox, 1996, 2002) than for supraliminal stimuli. Stronger effects for subliminal stimuli have been primarily observed in sub-clinically anxious groups. A possible explanation is that masked and unmasked stimuli initially have similar effects, but that the influence of unmasked stimuli is later counteracted by controlling processes (Fox, 1996). In contrast to non-clinically anxious individuals, anxiety patients often show similar attentional bias effects for masked and unmasked threat-related stimuli (e.g., Mogg, Bradley, et al., 1993; Mogg et al., 1995). It has been speculated that anxiety patients are less able to counteract initial automatic bias effects (Fox, 1996).

Several subliminal dot-probe studies (Mogg et al., 1995) and Stroop studies (MacLeod \& Rutherford, 1992; Mathews \& MacLeod, 1994; Bradley et al., 1995) during which word stimuli have been presented, report - in contrast to their supraliminal equivalent - a bias for all negative stimuli instead of content specific bias for threat-relevant information. A possible interpretation is that stimuli are first subjected to a rather coarse valence analysis (positive vs. negative) before they are consciously processed and evaluated in a more detailed way (e.g., Mogg \& Bradley, 1998; Dijksterhuis \& Aarts, 2003). In contrast, subliminal studies during which pictures instead of words have been presented, report content-specific bias effects and their results suggest that the observed effects depend on the mapping between stimulus content and an individual's main concerns (e.g., Mogg \& Bradley, 2002). Preconscious bias effects would depend on stimulus threat value, and, as a consequence, on the relevance of the stimulus for an individual's fears and previous experiences (Mogg \& Bradley, 2002).

The results of subliminal Stroop and dot-probe tasks have been taken as evidence of selective processing of threat-related stimuli that are not (fully) consciously perceived in anxiety. However, the interpretation of the reported findings is somewhat uncertain. Interestingly, in their meta-analysis of Stroop studies, Phaf and Kan (in press) could not find evidence for interference for subliminally presented threat words. In addition, for the subliminal Stroop and dot-probe tasks published until now, it is hard to ascertain that all participants were completely unaware of every (type of) stimulus, over the whole course of the task (cf. Mogg et al., 1995). Fixed exposure durations have been used, rather than determining the threshold for each individual subject and stimulus type separately. Recent research suggests however that individuals differ in their awareness of emotional faces with the same degree of

\footnotetext{
${ }^{9}$ However, not in all subliminal dot-probe studies, a significant attentional bias effect has been observed (Bradley et al., 1998).
} 
masking and that there is no fixed awareness threshold for emotional perception (Pessoa, Japee, \& Ungerleider, 2005). Furthermore, awareness checks have been presented only at a fixed time point during the study. However, awareness thresholds may vary across time (Holender, 1986). Furthermore, in the majority of these studies, conditions of restricted awareness and conditions of full awareness were randomly intermixed. Therefore it is not clear whether strategic or contextual factors, like expectation of threat or priming, were involved in conditions of restricted awareness (Fox, 1996).

\subsubsection{Visual Search Paradigms}

Visual search tasks have been designed to study pre-attentive selective processing of perceptually salient features (for a review, see Wolfe, 1998). Typically, participants are presented with a visual array and have to decide, by pressing a key, whether the target stimulus is among the presented stimuli. If the target differs from the distractors in one single basic feature (e.g., its color or orientation) then the time to detect this target does not depend on the number of distractors (flat search function) or on the exact position of the target in the array. This target seems to "pop out" from the display. The typical interpretation of such pop-out effects is that they are the outcome of pre-attentive, automatic processing of the target, independent of attentional resources (Treisman et al., 1992).

In the affective variant of the visual search paradigm, a threat-related stimulus is presented among non-threatening distractors, or vice versa, and participants have to decide whether all stimuli in the matrix belong to the same pre-defined category (odd-one-out), or whether a pre-defined target stimulus is present in the matrix. ${ }^{10}$ Öhman, Flykt, and Esteves (2001a) reported faster detection times for spider- and snake-targets presented among flowers or mushrooms, than vice versa. Spiders were faster detected by spider-fearful than by snake-fearful participants, while for snakes the reverse pattern was observed. Similarly, speeded detection times have been found for spiders among butterflies, especially in spider-fearful participants (Rinck, Reinecke, Ellwart, Heuer, \& Becker, 2005), for angry faces among neutral or happy faces (Hansen \& Hansen, 1988, but see Purcell, Stewart, \& Skov, 1996; Hampton, Purcell, Bersine, Hansen, \& Hansen, 1989; Öhman, Lundqvist, \& Esteves, 2001b), in high-trait-anxious (Byrne \& Eysenck, 1995) and in social phobics (GilbaoSchechtman, Foa \& Amir, 1999). Besides speeded detection of threat-related targets, also slower detection of neutral targets among threat-related distracters has

\footnotetext{
${ }^{10}$ The outcome of visual search studies may depend on which of these instructions is given. Rinck and colleagues (Rinck et al., 2005; Rinck \& Becker, 2005; Rinck, Becker, Kellermann, \& Roth, 2003) observed increased distraction by threat with both variants, but speeded threat detection only in the odd-one-out variant. A more detailed discussion of this issue is beyond the scope of the present chapter.
} 


\section{Chapter 1}

been observed (e.g., Rinck et al., 2005), suggesting increased distraction by threat and slowed disengagement from it.

Furthermore, Öhman and colleagues (2001a) reported that the detection time for spiders, snakes and angry faces did not depend on the number of distracters (flat search function). Others have found that angry (schematic) faces are detected faster and more efficiently than happy faces, but do not necessarily "pop out" from the display (a smaller but not flat search function; e.g. Fox et al., 2000; Eastwood, Smilek, \& Merikle, 2001), suggesting that angry faces are not pre-attentively detected. Furthermore, it is debated whether pop-out effects are specific for threatrelated stimuli. Pop-out effects have also been reported for, for example, pictures of non-threatening animals and fruit, at least in the general population (not screened for anxiety or fear; Tipples, Young, Quinlan, Brooks, \& Ellis, 2002; Lipp, Derakshan, Waters, \& Logies, 2004).

Finally, note that in all visual search studies cited so far, the emotional stimuli were task-relevant. Very recently, Eimer and Kiss (2007) provided electrophysiological evidence for selective processing of a fearful face among neutral faces, even when this face was completely task-irrelevant and attention was actively focused elsewhere.

\subsubsection{Experimental Paradigms: Taken Together}

In the previous paragraphs, some of the major behavioral paradigms used so far to study (automatic) preferential processing of visual emotional stimuli were discussed. Interference tasks, dot-probe tasks, subliminal versions of these paradigms, and visual search tasks were addressed. By using these tasks, significant advances have been made in our understanding of the mechanisms associated with anxiety and there is a growing body of evidence suggesting that anxiety is characterized by automatic preferential processing of threat-relevant information. Recently, BarHaim and colleagues (Bar-Haim, Lamy, Pergamin, Bakermans-Kranenburg, \& van IJzendoorn, 2007) subjected the available empirical data on threat-related attentional bias, as measured with the emotional Stroop task, dot-probe task, or modified exogenous cueing task, to a meta-analysis. ${ }^{11}$ The results of this analysis suggest that threat-related bias is a robust phenomenon in anxious individuals (low-to-medium effect size) and that this bias does not exist in non-anxious individuals, neither for

\footnotetext{
${ }^{11}$ Altogether, 172 studies were included in this first meta-analysis, all published between February 1986 and May 2005. Studies using the visual search task were not included, because in May 2005, there were insufficient visual search studies (meeting the inclusion criteria) to allow systematic analysis (Bar-Haim et al., 2007, p. 6).
} 
threat-related pictures nor for words. ${ }^{12}$ Second, the results indicate that anxious individuals do not only show a bias for supraliminally, but also for subliminally perceived threat. Emotional Stroop interference effects were larger with supraliminal than with subliminal exposure (cf. Phaf \& Kan, in press), whereas the reverse was true for dot-probe effects. According to Bar-Haim et al. (p. 15), this pattern of results is in line with the idea that Stroop effects reflect relatively late, controlled processes, whereas dot-probe effects reflect earlier attentional processes. Third, the bias effects elicited by threat-related pictures and words were equivalent in effect size. However, when only considering effects measured with subliminal stimulus exposure, effects were larger for pictures than for words. Meanwhile, Bar-Haim et al. stated that this latter finding might reflect the difference between Stroop and dotprobe effects for subliminally presented stimuli as described above, for in most dotprobe studies pictures were used, whereas in most Stroop studies words. Fourth, anxiety patients and non-clinical individuals reporting high levels of anxiety did show bias effects with a similar effect size. More specifically, clinically anxious individuals showed a larger bias with supraliminal than with subliminal exposure duration, whereas non-clinically anxious participants showed no difference between these two exposure conditions. According to Bar-Haim et al., also this latter finding might reflect the difference between Stroop and dot-probe effects for subliminally presented stimuli, for in studies with clinical samples, the emotional Stroop task was more often used than the dot-probe task.

Notwithstanding their substantial contribution, some difficulties and limitations have been associated with (subliminal) interference tasks, dot-probe tasks, and visual search tasks as used in previous research. These limitations have been a source of inspiration for the present dissertation work.

Finally, all the research reviewed above and also the studies conducted for the present dissertation project are correlational in nature. In other words, these experiments show associations between anxiety and the existence of some specific bias effects. However, they reveal nothing about the direction of the causal relationship between anxiety and these effects. Does this cognitive bias play a causal role in the etiology and/or maintenance of anxiety (disorders)? Only very recently, researchers have started to address this question, for example by training people to have an attentional bias towards or away from negative stimuli in a modified dot-probe task and to assess whether these training groups differ in their emotional responses to stress (e.g., MacLeod, Rutherford, Campbell, Ebsworthy, \& Holker, 2002; Mathews \& MacLeod, 2002).

\footnotetext{
${ }^{12}$ In their meta-analysis, Bar-Haim et al. (2007) could not test the hypothesis that non-anxious individuals show a bias only with high threat intensity levels (e.g., Wilson \& MacLeod, 2003), because a comparison of stimuli's threat levels across studies was impossible (p. 15).
} 


\subsection{Preferential Processing of Emotional Information as Revealed by Simon Effects}

In this last section, a new reaction-time paradigm is introduced, developed to study early preferential processing of emotionally relevant information. We feel that, given the limitations of some of the existing paradigms, there is room for the development of new approaches that can be used to obtain additional evidence for preferential processing of emotional stimuli. The new paradigm was applied for the first time in the experiments presented in Chapters 3, 4, and 5, to examine early preferential processing of threat in sub-clinical and clinical anxiety. In the paragraphs that follow, first the underlying idea is described, and then the behavioral task. Finally, the novel paradigm is compared with existing tasks, like the Stroop task, dot-probe task, and Affective Simon Paradigm.

\subsubsection{Simon Effects Elicited by Preferentially Processed Stimuli}

The basic idea of the novel approach is as follows. If an emotional stimulus, presented together with a neutral stimulus, is preferentially processed, it may act as if it is actually presented alone. If so, this emotional stimulus should stand out of the display and have similar effects on responses as a single peripheral stimulus.

Single peripheral stimuli are known to yield a Simon effect (reviews by Simon, 1990; Lu \& Proctor, 1995). Simon effects arise when we have to make a spatially arranged response, such as pressing a left or a right key, in response to a non-spatial feature of a peripheral stimulus, for example its form or color. Although the spatial position of the peripheral stimulus is irrelevant to the task at hand, its correspondence with the position of the correct response results in better task performance (faster responses, less errors), compared to when they do not correspond. In one typical Simon task (e.g., Craft \& Simon, 1970; Hedge \& Marsh, 1975), a red or green light is presented on either the left or right side of a central cross. Participants have to press the right key on a response box in response to a red stimulus, and the left key in response to a green one. On this task, responses are consistently faster to a red stimulus (right response) on the right than to a red stimulus on the left, and to a green stimulus (left response) on the left than to a green stimulus on the right. Similar effects have been reported when stimulus and response positions were oriented vertically (i.e., top/bottom positions; Nicoletti \& Umiltà, 1984).

Thus, single peripheral stimuli typically induce a Simon effect. Suppose now, that a peripheral stimulus is not presented alone, but simultaneously with another one, and suppose that the first stimulus is, for one reason or another, more efficiently processed than the second one. Then, we would expect that the first stimulus 
also elicits a Simon effect: faster responses when its position corresponds with the position of the correct response than when these positions do not correspond. If we turn this line of reasoning the other way around, finding a Simon effect for a certain stimulus that is presented concurrently with another stimulus would indicate that that certain stimulus was preferentially (or at least more efficiently) processed than the other one. So, finding a significant Simon effect for an emotional stimulus that is presented together with a comparable but emotionally neutral stimulus would suggest that this emotional stimulus was preferentially processed. We argue that such a finding would add evidence to the hypothesis of preferential processing of the emotional stimulus, since emotional content is the only difference between the two stimuli.

\subsubsection{Spatial Affective Simon Task (SAST)}

To study Simon effects for emotional stimuli presented together with a neutral stimulus, we designed an affective variant on the classical Simon task. During this Spatial Affective Simon Task (SAST), at critical trials, one neutral and one emotional word are presented at two fixed positions on the screen, for example above and below central fixation. Response position, for example top or bottom key, is determined by a non-spatial stimulus feature available from both words, for example the print color of the word pair (e.g., press top key when both words printed in red, bottom key when both in green). Correspondence of irrelevant emotional stimulus position and response position varies across trials. For example, on half of the trials that the emotional word is on the top position, color information indicates that the top key has to be pressed; on the other half the bottom key has to be pressed.

Remember that in the original Simon task as described above, the position of the single stimulus is the irrelevant stimulus feature and its print color the relevant stimulus feature. Likewise, in the SAST, the position of the emotional stimulus within the stimulus pair is the irrelevant feature and color of the stimulus pair the relevant feature. To our knowledge, there is no direct equivalent of the SAST in which the stimuli differ in a physical feature (e.g., form, orientation), rather than in emotional content. In such a basic SAST equivalent, a salient stimulus (expected to result in a pop-out) would be presented together with at least one less salient stimulus, (1) with both stimuli carrying the relevant information (e.g., print color), (2) with the feature dimension on which the stimuli differ from each other not being 


\section{Chapter 1}

the task-relevant dimension (e.g., form or orientation) and (3) with as irrelevant feature the position of the salient stimulus within the pair. ${ }^{13}$

In a similar way as has been done with dot-probe tasks, SAST parameters can be modified in order to examine, for example, the content-specificity, time-course, and automaticity of the underlying processes. For instance, different types of stimuli can be presented (e.g., pictures instead of words; stimuli differing in threat intensity level or current relevance), stimulus exposure duration and interval between stimulus onset and onset of the relevant information can be varied, and difficulty of the discrimination to be made can be manipulated so as to delay responses and tap the effects later in time. In the first series of SAST experiments, presented in Chapters 3-5, word stimuli were presented for 14 (followed by backward mask) or $500 \mathrm{~ms}$ (similar exposure durations as in dot-probe literature), and participants had to decide whether the words were presented in red or in green, an easy discrimination.

\subsubsection{SAST versus Other Paradigms}

The reader may wonder in what way the SAST actually differs from other tasks developed to measure preferential processing of emotionally relevant information, including the emotional Stroop task and the dot-probe task. Furthermore, other affective variants of the traditional Simon task have been proposed. In what way does the SAST differ from these previous affective Simon tasks? Table 1.2. provides a summary of the paradigms.

\subsubsection{SAST versus Emotional Stroop}

At a superficial level, the SAST may look similar to the emotional Stroop task, for in both tasks words are printed in different colors and participants have to respond to this color, while ignoring word meaning. However, at a structural level, the tasks are completely different. In contrast to the SAST, emotional Stroop tasks do not involve any kind of manipulation of spatial stimulus-response correspondence. The logic underlying emotional Stroop tasks is that preferential processing of the meaning of emotional words would interfere with performance on a primary task. So, preferential processing of emotional word meaning is derived from worse performance on a main task when emotional stimuli are presented than when neutral stimuli are presented. In the SAST, preferential processing of emotional over neutral

\footnotetext{
${ }^{13}$ There are Simon studies during which a target stimulus was presented simultaneously with another stimulus, a reference object (e.g., Hommel, 1993a) or a filler noise stimulus (e.g., Wascher \& Wauschkuhn, 1996). However, in these tasks, only the target stimulus conveyed the task-relevant information.
} 
stimuli is derived from better performance when emotional stimulus and response spatially correspond than when they do not correspond.

\subsubsection{SAST versus Dot-Probe Task}

At critical trials of both the dot-probe task and the SAST, stimulus pairs consisting of one emotional and one neutral stimulus are presented. In dot-probe literature, the focus has been on the spatial correspondence of emotional word and probe. Faster responses to probes taking the position of an emotional stimulus than to probes taking the position of a neutral stimulus have been taken to reflect attentional bias for the emotional stimulus. In this literature, no attention has been paid to the possible effects of spatial correspondence of emotional word and response. Note, however, that in dot-probe tasks during which a forced-choice response is required (i.e., probe-position or -classification tasks), not only the spatial correspondence of emotional stimulus and probe, but also the spatial correspondence of emotional stimulus and response is or can be manipulated (see Table 6.1., Chapter 6).

As already mentioned before (see Section 1.2.2.3.), in probe-position tasks, the spatial correspondence of emotional stimulus and probe (attentional bias) is confounded with spatial correspondence of emotional stimulus and response (called response bias), because in these tasks, there is, by instruction, always a spatial correspondence of probe and correct response (e.g., press left key to left probes on the left, right key to probes on the right). In probe-classification tasks, however, attentional bias and response bias are not confounded, for the spatial correspondence of emotional stimulus, probe and response is completely balanced throughout trials. This idea is further elaborated in Chapter 6. We argue that probe-classification tasks can be used to study both attentional bias and response bias.

There are two important structural differences between the SAST and a typical dot-probe task. First, during a typical dot-probe task, responses are delayed compared to the emotional stimulus. That is, responses are given to a probe following the offset of the emotional stimulus. However, during the SAST as described above, task-relevant information is already available at word onset. So, any preferential processing is measured as soon as the emotional stimulus appears and not after a delay as in a dot-probe task. 
Chapter 1

Table 1.2.

\begin{tabular}{|c|c|c|}
\hline Task & Stimuli & Response \\
\hline Stroop color-word task & $\begin{array}{l}\text { Centrally presented color word } \\
\text { or meaningless stimulus (e.g., sequence } \\
\text { of Xs) printed in color }\end{array}$ & Naming print color \\
\hline Emotional Stroop task & $\begin{array}{l}\text { Centrally presented emotional or } \\
\text { neutral word printed in color }\end{array}$ & Naming print color \\
\hline Exogenous cueing task & $\begin{array}{l}\text { Peripheral or non-spatial cue, followed } \\
\text { by a peripheral probe }\end{array}$ & $\begin{array}{l}\text { Probe detection by key press or } \\
\text { forced-choice response based on } \\
\text { probe position/type by pressing one } \\
\text { of the alternative keys }\end{array}$ \\
\hline $\begin{array}{l}\text { Emotional exogenous } \\
\text { cueing task }\end{array}$ & $\begin{array}{l}\text { Peripheral emotional or neutral } \\
\text { stimulus, followed by a peripheral } \\
\text { probe }\end{array}$ & $\begin{array}{l}\text { Probe detection by key press or } \\
\text { forced-choice response based on } \\
\text { probe position/type by pressing one } \\
\text { of the alternative keys }\end{array}$ \\
\hline Dot-probe task & $\begin{array}{l}\text { Peripheral emotional stimulus together } \\
\text { with neutral stimulus, followed by a } \\
\text { peripheral probe }\end{array}$ & $\begin{array}{l}\text { Probe detection by key press or } \\
\text { forced-choice response based on } \\
\text { probe position/type by pressing one } \\
\text { of the alternative keys }\end{array}$ \\
\hline
\end{tabular}

Classical Simon task Peripheral stimulus

Affective Simon task

Spatial affective Simon task (SAST)
Peripheral emotional stimulus together with neutral stimulus, printed in same color
Forced-choice response based on non-spatial stimulus feature by pressing one of the alternative spatially oriented keys

Centrally presented emotional stimulus

Forced-choice response based on non-affective stimulus feature by giving one of the alternative affective (verbal or non-verbal) responses

Forced-choice response based on non-spatial stimulus feature (color) by pressing one of the alternative spatially oriented keys 
Table 1.2.

Used to measure
Interference due to processing of

task-irrelevant, intrusive stimuli

Selective processing of emotional stimulus content

Reflexive orienting of spatial attention

Effect emotional stimulus content on attentional disengagement

Effect emotional stimulus content on attentional engagement / shifting

Selective attention bias for emotional stimulus, relative to neutral stimulus

\section{Relevant comparisons between conditions}

Performance when word meaning and print color do not correspond vs. performance when meaningless stimuli (interference)

Performance when word meaning and print color correspond vs. performance when meaningless stimuli (facilitation)

Performance when word meaning and print color do not correspond vs. when they correspond

Performance for emotional words vs. performance for neutral words
Performance when peripheral cue and probe do not spatially correspond

vs. performance when neutral cue (cost)

Performance when peripheral cue and probe spatially correspond vs. performance when neutral cue (benefit)

Performance when peripheral cue and probe do not spatially correspond

vs. when they do not correspond (overall attentional orienting effect)

Performance when emotional stimulus and probe do not spatially correspond

vs. when neutral stimulus and probe do not spatially correspond

Performance when emotional stimulus and probe spatially correspond vs. when neutral stimulus and probe spatially correspond

Performance when emotional stimulus and probe spatially correspond vs. when they do not correspond
Interference due to tendency to respond to stimulus in a spatially corresponding manner (response bias)

Automatic processing of stimulus valence

Selective response bias for emotional stimulus, relative to neutral stimulus
Performance when stimulus and response spatially correspond vs. when they do not correspond

Performance when stimulus valence and response valence correspond vs. when they do not correspond

Performance when emotional stimulus and response spatially correspond

vs. when they do not correspond 


\section{Chapter 1}

We conducted a probe-classification study during which we tapped attentional bias at two moments in its time-course: at word onset, as in the SAST, and after a delay of $500 \mathrm{~ms}$ (see Chapter 7).

Second, during dot-probe tasks, task-relevant information (conveyed by the probe) is presented either at the presumed attended location (emotional stimulus position) or at the presumed unattended location (neutral stimulus position). Because relevant information is only available at one of the locations, probeclassification tasks can be used to study attentional bias, independent of response bias. During the SAST, task-relevant information is always present at both locations; the emotional as well as the neutral stimulus carry this information (e.g., both stimuli printed in the relevant color). Therefore, in the SAST, an attentional shift alone should not lead to faster reactions with one of the response keys. In our view, there can only be a contribution of attentional bias in the SAST in the sense that an attentional bias may lead to a response bias. The SAST is a simple task, with a less complicated structure than a probe-classification task (i.e., less contrasts included ${ }^{14}$ ), providing a pure measure of response bias.

\subsubsection{SAST versus Other Affective Simon Tasks}

In other affective variants of the Simon task (e.g., De Houwer \& Eelen, 1998; De Houwer, Crombez, Baeyens, \& Hermans, 2001), the relationship between the taskirrelevant affective content of centrally presented stimuli and the valence of the correct response (e.g., say 'positive' or 'negative') has been manipulated in order to investigate automatic affective processing. ${ }^{15}$ So, in these tasks, Simon effects (i.e., irrelevant stimulus-response compatibility effects) have been defined in terms of stimulus valence, rather than in terms of spatial position. Neither stimulus nor response had any spatial relevance at all.

In contrast, our modification of the Simon paradigm manipulates spatial correspondence, much like in the original Simon paradigm. In fact, this was the reason for us to name our task the Spatial Affective Simon task (SAST), to acknowledge the difference with previous affective Simon tasks. In the SAST, affective stimulus content is presented lateralized. That is, emotional words always appear on the display opposite a neutral word (e.g., one above the other). We deliberately avoid responses to have an intrinsic affective meaning, and focus solely on the spatial

\footnotetext{
${ }^{14}$ That is, only spatial correspondence of emotional stimulus and response is varied, and not the spatial correspondence of emotional stimulus and an extra probing stimulus, and the spatial correspondence of such a probe and response.

${ }^{15}$ Also in tasks designed to study approach-avoidance response tendencies, affective correspondence between emotional stimulus and response (e.g., push/pull lever, press/release button, arm tension/flexion) has been manipulated (e.g., Solarz, 1960; Sobotka, Davidson, \& Senulis, 1992; Chen \& Bargh, 1999; Wentura, Rothermund, \& Bak, 2000; Cacioppo, Priester, \& Berntson, 1993; Neumann, Főrster, \& Strack, 2003).
} 
relationship between emotional content and response. This is regarded as the crucial innovative feature of the SAST. Because the SAST is structurally highly different from previous affective Simon paradigms, it likely taps an entirely different mechanism. 



\section{Chapter 2 \\ Outline of the Empirical Chapters}


The goal of the present dissertation was to contribute to a better understanding of preferential processing of threat-relevant information associated with anxiety. The two main themes addressed were (1) biased processing in anxiety as evidenced by spatial affective Simon effects, and (2) the dynamics of anxiety-related attentional bias effects. Five empirical studies were conducted, reported in Chapters 3-7. These empirical chapters as well as the final general discussion in Chapter 8 can be read in isolation.

An important part of the work was dedicated to the development and application of the 'spatial affective Simon approach' (Chapter 1, Section 1.3). The first three chapters describe studies in which the SAST paradigm (Chapter 1, Section 1.3.2.) was used to investigate early preferential processing of threat-relevant information in undergraduates exposed to a physical stressor (height; Chapter 3), in undergraduates with a high-anxious personality trait (Chapter 4), and in an anxious clinical sample (Chapter 5). In the study reported in Chapter 6, a probeclassification task was used to assess both spatial affective Simon effects (called 'response bias') and attentional bias.

The temporal characteristics of anxiety-related bias effects were studied in Chapters 6-7. In both experiments, high- and low-trait-anxious undergraduates performed a probe-classification task. In Chapter 6, it is argued that analyses of reaction time distributions may help to reveal the temporal course of the supposed attentional bias effects. In the experiment in Chapter 7, the time-course of attentional bias and the influence of processing time were studied. This was done by independently manipulating the moment at which the bias was tapped and exposure duration of the stimuli, respectively.

Chapter 8 reviews the findings in the preceding chapters, and addresses several issues on interpretation and methodology. Recommendations and suggestions for further research are given. In addition, some outstanding questions and 'hot' issues in research on preferential processing in anxiety today, that were not directly addressed in the present experiments, are described and some ideas for future research are given. 


\section{Chapter 3 \\ A Simon Effect for Threat-Related Stimulus Content}

This chapter is published as: Schrooten, M. G. S., \& Smulders, F. T. Y. (2007). A Simon effect for threat-related stimulus content. Journal of Behavior Therapy and Experimental Psychiatry, 38, 121-132. 


\subsection{Abstract}

When a threat-related stimulus is preferentially processed, it may act as if it is presented alone, and thus trigger processes comparable with the effects elicited by a single stimulus. Peripheral stimuli are known to yield a Simon effect: faster responses when stimulus and response spatially correspond than when not. We designed a task during which a threat-related (physical-threat, social-threat, or height) word is presented with a neutral word (14 or $500 \mathrm{~ms}$, masked), one above the other, and spatial correspondence of threat-related word and response varies across trials. Undergraduates performed this task when exposed to height $(N=22)$ or in a lab $(N=25)$. The height group, compared to the control group, showed a contentspecific Simon effect for physical-threat words. This result adds evidence to the hypothesis of preferential processing of relevant threat-related information.

\subsection{Introduction}

Early preferential processing of threat is thought to play a crucial role in the development and maintenance of anxiety (fear) (cf. Williams, Watts, MacLeod, \& Mathews, 1997). This is in line with an evolutionary perspective, stating that the adaptive function of anxiety is the automatic detection of threat so as to react quickly and efficiently when necessary (Öhman, 1994). Modified versions of the Stroop task, spatial-orienting task, and visual search paradigm have been used to study this preferential processing.

Emotional Stroop experiments have demonstrated that anxious, compared to non-anxious, individuals are slower in naming the color of words related to their worries or to relevant threat than in naming the color of other words (for review, see Williams, Mathews, \& MacLeod, 1996).

Modified spatial-orienting tasks were developed to assess the spatial distribution of attention. During the dot-probe task (MacLeod, Mathews, \& Tata, 1986), for example, one neutral and one emotional word or picture are presented. After these stimuli disappear, a probe appears at a location previously occupied by one of them. Faster responses when emotional stimulus and probe occur in the same position than when they appear in different positions should reflect preferential orienting to the (location of the) emotional stimulus (i.e., attentional bias). Typically, anxious people show an attentional bias towards threat-related words and pictures related to the content of their anxiety (for reviews, see Williams et al., 1997; Mogg \& Bradley, 1998).

To examine whether preferential processing occurs automatically and preconsciously, Stroop and dot-probe tasks have been used in which stimuli have been presented under conditions of restricted awareness, below the perceptual threshold. 
Several studies provide evidence suggesting selective processing of such subliminally presented threat-related stimuli (for review, see Mogg \& Bradley, 1998). Further evidence for automatic processing of threat comes from visual search tasks. Detection times were faster for threat-related targets presented among non-threatening distractors than vice versa, especially in fearful individuals (e.g., Öhman, Flykt, \& Esteves, 2001). Furthermore, Öhman and colleagues (2001) reported that detection time for spiders and snakes did not depend on the number of distractors (flat search function). The typical interpretation of such "pop-out" effects is that they are the outcome of pre-attentive, automatic processing of the target (Treisman, Vieira, \& Hayes, 1992).

The above experimental paradigms have been influential in our understanding of the cognitive concomitants of affective processes, but have also been criticized. Although emotional Stroop effects have been frequently observed, it is unclear which underlying processes are involved (e.g., De Ruiter \& Brosschot, 1994; Williams et al., 1996). Emotional Stroop effects might, for example, be due to nonspatial attention towards emotional word content, later cognitive avoidance, or response inhibition. In addition, it has been found that the largest portion of the emotional Stroop effect is operating between rather than within trials (McKenna \& Sharma, 2004), raising debate about the immediacy of the interference.

As mentioned above, in dot-probe studies relatively fast responses when threatrelated stimulus and probe spatially correspond have typically been taken to reflect attentional orienting towards (the location of) the threat-related stimulus. However, this pattern of results may also be due to difficulty in disengaging attention from threat-related stimuli. Recent exogenous cueing experiments suggest that highanxious people especially have difficulty in disengaging attention from threat-related stimuli (for review, see Fox, 2004). Note that during dot-probe tasks the imperative probe is always presented after the stimulus pair disappeared. By varying the time interval between the onset of the stimulus pair and the onset of the probe, attentional bias can be tapped at various moments in its time-course (e.g., Mogg, Bradley, De Bono, \& Painter, 1997), but attention is always tapped when the emotional stimulus has already disappeared.

Subliminal versions of the Stroop and dot-probe task did also not remain free from criticism. For instance, it has been argued that for subliminal Stroop and dotprobe tasks published until now it is difficult to ensure that participants were completely unaware of every (type of) stimulus over the whole course of the task (cf. Mogg, Bradley, \& Williams, 1995).

Finally, with regard to visual search tasks, it has been found that angry (schematic) faces are detected faster and more efficiently than happy faces, but do not necessarily "pop-out" the display (a smaller, but not flat search slope; e.g., Fox et al., 2000; Eastwood, Smilek, \& Merikle, 2001), suggesting that angry faces are not preattentively detected. Furthermore, it is debated whether pop-out effects are specific 
for threat-related stimuli. Pop-out effects have also been reported for, for example, pictures of non-threatening animals and fruit, at least in the general population (Tipples, Young, Quinlan, Broks, \& Ellis, 2002).

The aim of the present study was to further investigate preferential processing of threat by applying a new approach. The underlying idea is that sudden peripheral onset of preferentially processed visual stimuli (cf. pop-out) may trigger processes that are similar to the effects of presenting a single peripheral stimulus. More specifically, if an emotional stimulus, when presented together with neutral stimuli, is preferentially processed, than this emotional stimulus may 'pop-out' of the display and act as if it is presented alone. Single peripheral stimuli are known to yield a Simon effect: faster responses when the irrelevant stimulus position corresponds to response position than when not (for review, see Lu \& Proctor, 1995). In one typical Simon task (Hedge \& Marsh, 1975) a red or green stimulus is presented on the left or right side of a central cross. Participants press the right key on a response box to red, the left key to green. They are faster to a red stimulus (right response) on the right than to a red stimulus on the left, and to a green stimulus (left response) on the left than to a green stimulus on the right. So, although stimulus position is irrelevant to the instructed task, responses are faster if stimulus and response are spatially corresponding than when not. Similar effects have been reported when stimuli and responses are vertically arranged (Nicoletti \& Umiltà, 1984).

Finding a Simon effect for threat-related stimuli presented simultaneously with a neutral stimulus (i.e., faster responses when the position of the threat-related stimulus and response position correspond than when they do not) would add evidence to the hypothesis of preferential processing of threat, since threat-content is the only difference between the stimuli. To investigate Simon effects for emotional stimuli, we designed a task during which an emotional and a neutral stimulus are presented, and the correspondence of irrelevant emotional stimulus position and response position varies across trials. Participants responded to a feature of the stimuli themselves (i.e., the print color of the stimulus pair). By applying this novel paradigm, we aim to provide converging evidence for preferential processing of threat. Furthermore, with our paradigm, any preferential processing is measured as soon as the emotional stimulus appears and not after a delay as in the dot-probe task.

In the present experiment, we applied our task to explore preferential processing of physical-threat words in a physically threatening situation. One group of participants was exposed to height; the control group was tested in a normal lab. It was hypothesized that participants exposed to height would show a Simon effect for words related to physical-threat, while the control group would show no, or a significantly smaller Simon effect. In addition, we anticipated that the height group might also have a bias for words semantically related to their specific experimental situation (i.e., height) (cf. MacLeod \& Mathews, 1988). Furthermore, social-threat 
words were included to assess whether participants in a physically-threatening situation show a Simon effect for threat-relevant stimuli, or threat stimuli in general. Finally, in order to investigate whether preferential processing of threat-related stimulus content is automatic in the sense that it also occurs in conditions of restricted awareness, trials on which masked words were presented for $14 \mathrm{~ms}$ were intermixed with trials with $500 \mathrm{~ms}$ presentation. Contrast-masking studies show that spatial responses can be influenced by invisible, masked stimuli (Vorberg, Mattler, Heinecke, Schmidt, \& Schwarzbach, 2003).

\subsection{Method}

\subsubsection{Participants}

Forty-seven students at Maastricht University (mean age $=23$ years, $S D=2.07$ ) participated on an informed consent basis to partially fulfill course requirements. Immediately prior to the experiment, participants completed the trait-anxiety subscale of the State-Trait Anxiety Inventory (STAI; Van der Ploeg, Defares, \& Spielberger, 1980). They were assigned to the height condition $(N=22 ; 7$ men $)$ or the control condition $(N=25 ; 7$ men) in such a way that the groups did not significantly differ in mean STAI-trait scores (Height: $M=32.36, S D=5.75$, range $=22$ - 48; Control: $M=32.52, S D=6.17$, range $=20-46 ; t(45)=.09, p=.9)$. All participants were native Dutch speakers, non-dyslexic, and had (corrected to) normal vision and color vision. Approval was obtained from the local ethics committee.

\subsubsection{Stimuli}

The critical stimuli consisted of 32 physical-threat (e.g., wound, cancer, ambulance), 32 social-threat (e.g., stupid, shame, criticism), and 32 height-related (e.g., height, ravine, abyss) Dutch words, individually matched with neutral words in terms of length and frequency (CELEX; Baaijen, Piepenbrock, \& Gulikers, 1995). Ninety-six neutral-neutral pairs were created, each pair matched for letter length, to act as filler material. Word length ranged from three to ten letters. Physical-threat and social-threat words were drawn from published lists (e.g., MacLeod et al., 1986). The affective content of these words, the neutral words paired to them, and the filler words was rated by eight staff members $(3 \mathrm{men})$ on an electronic visual analogue scale. Physical-threat words, social-threat words, and neutral words paired to them were rated as to whether they were associated with physical-threat and 
social-threat, respectively (from 'not at all' to 'extremely'). The valence of the filler words was rated on a bipolar scale (from 'very negative' to 'very positive'). The mean rating difference ( $z$-scores) between the members of any physical-threat-neutral pair was at least $1.3(M=1.9)$, of any social-threat-neutral pair at least $1.4(M=1.8)$, and of any neutral-neutral pair between -.5 and $.5(M=.02)$. During practice trials, words were presented that were not presented during experimental trials. Mirrored, upside-down upper-case consonants, numbers, and symbols were used as backward mask. Masks were unique to each word, length-matched and color-matched with the preceding word, and presented for $14 \mathrm{~ms}$.

\subsubsection{Apparatus}

Presentation of the movie and the task was controlled by a Dell Latitude D600 laptop, connected to a 17 -inch CRT screen. ERTSVIPL V3.32c (Beringer, 1987) was used to control stimulus presentation, and to record reaction times (RTs).

\subsubsection{Procedure}

The participants were seated at a viewing distance of about $60 \mathrm{~cm}$ from the monitor. In the height condition, participants were sitting on a stage on a walking bridge (approximately $12 \mathrm{~m}$ high, glass walls from floor to ceiling), close to an open window. In the control condition, participants were seated in a testing room without windows. Bright lamps lit the room to mimic the daylight on the bridge. All participants wore earflaps to exclude outside noise. They were told that the study investigated the relationship between concentration and performance. The real purpose was explained after all participants had been tested.

To further augment the feeling of physical threat, participants in the height condition first viewed a 6-minute extract from the movie 'Cliffhanger' (Marshall \& Harlin, 1993), showing a climber falling into a ravine. The control group viewed a non-threatening 6-minute extract from 'Finding Nemo' (Walters \& Unkrich, 2003). Immediately afterwards, mood state was measured by using a Dutch shortened (30 items) Profile of Mood States questionnaire (POMS; Wald \& Mellenbergh, 1990). Then, the participants performed the computerized task.

The typical trial configuration is depicted in Figure 3.1. 


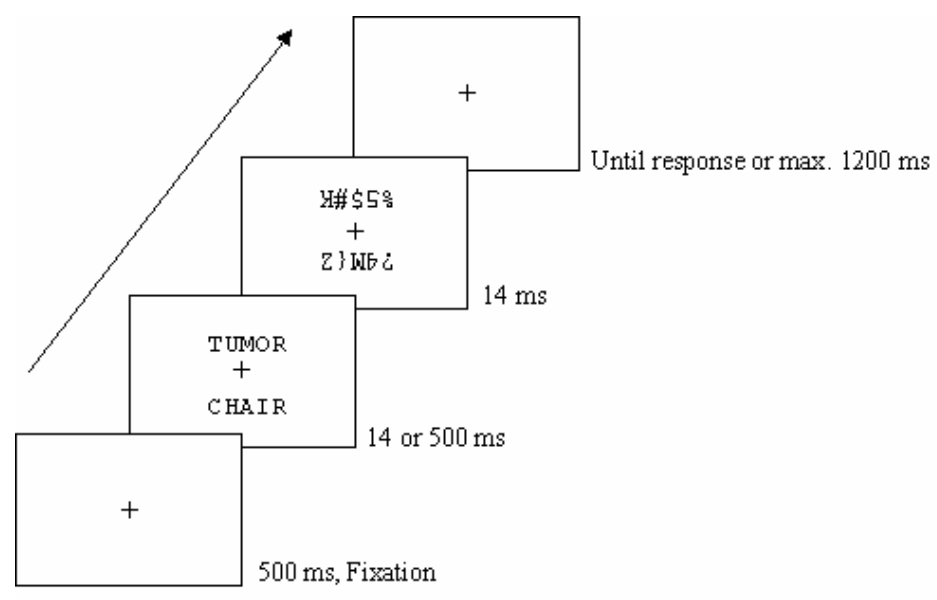

Figure 3.1. Typical trial configuration. The word pair was printed in red or green. The fixation cross took on the color of the word pair and the masks were color-matched with the preceding word pair.

Each trial started with a white central fixation cross $(500 \mathrm{~ms})$. Then, a word pair in upper-case letters (non-proportional font, $0.5 \mathrm{~cm}$ high) appeared on a dark background with one word above, the other below the center of the screen $(3 \mathrm{~cm}$ apart). In half of the trials, both words were printed in red (Red Green Blue (RGB) color model: 160, 0, 0), in the other half of the trials in green (RGB: 0, 152, 0). The cross took on the color of the words. In half of the trials, words appeared for 14 $\mathrm{ms}$, in the other half for $500 \mathrm{~ms}$ (commonly used durations in dot-probe studies). In both exposure conditions, words were followed by a mask. All participants were instructed to respond as quickly and accurately as possible by pressing the top key on a response box with the right index finger to red, and the bottom key with the left index finger to green. No instruction was given to process the words in any particular way. Participants had to fixate the cross at all times. The cross remained on the screen until the participant responded, or for maximum $1200 \mathrm{~ms}$. In case of an error, a beep and an error message (showing the instruction) lasting $250 \mathrm{~ms}(+$ $1500 \mathrm{~ms}$ pause) were inserted. Visual feedback was given if the participant responded too slowly (RT $>800 \mathrm{~ms}$ ) or prematurely (RT $<150 \mathrm{~ms})$. The inter-trial interval was $500 \mathrm{~ms}$.

All participants completed two task blocks. Each block consisted of 36 practice trials, followed by 384 experimental trials. Per block, there were an equal number of experimental trials for each combination of type of word pair, exposure duration, 
position of the critical word, and response position. Each word pair appeared once in each exposure duration. The experimental trials were presented in a different random order for each participant. Performance feedback (mean RT) was given every 32 trials during breaks terminated by the participants. The height group had to throw a pellet out of the window during the $2^{\text {nd }}, 7^{\text {th }}, 12^{\text {th }}, 17^{\text {th }}$, and $22^{\text {nd }}$ break, and count how many seconds it took before it touched the ground. The control group had to throw a pellet three times in a basket, and count how many times it was in.

\subsection{Results}

\subsubsection{Group Characteristics}

The height group scored higher on the POMS tension-anxiety subscale than the control group (Mann-Whitney $U=162, p=.016$ ), suggesting that our manipulations were successful. Furthermore, the height group scored higher on the depression subscale $(U=175, p=.004)$, and lower on the vigor subscale $(U=179, p=$ $.04)$ than the control group. This group difference may be explained by the fact that the height group viewed a movie fragment that ended dramatically, whereas the control group watched a cheerful cartoon. Both groups reported comparable levels of fatigue and anger $(U=247$ and 221 , respectively, $n s$.).

\subsubsection{Behavioral Results}

The result section focuses on RT analyses, for no significant Simon effects were observed for error rates. All p-values are two-tailed.

Median RTs were first subjected to an overall mixed design ANOVA with critical word position (top, bottom), response position (top, bottom), type (physical-threat words, social-threat words, height words), duration $(14 \mathrm{~ms}, 500 \mathrm{~ms}$ exposure), and block (first, second task block) as within-subjects factors, and group (height, control) as between-subjects factor. The reason for using median rather than mean RTs was to reduce the potential influence of outliers. Table 3.1. shows median RTs in each experimental condition.

RT was slower in the height $(363 \mathrm{~ms})$ than in the control group (336 ms), $F(1$, $45)=14.5, p<.0005$. In addition, RT was slightly faster in the second (347 ms) than in the first block (352 ms), $F(1,45)=6.5, p=.015$. Relevant for the hypotheses are the results involving an interaction between critical word position and response position, reflecting Simon effects. There was a significant type $\mathrm{x}$ critical word 


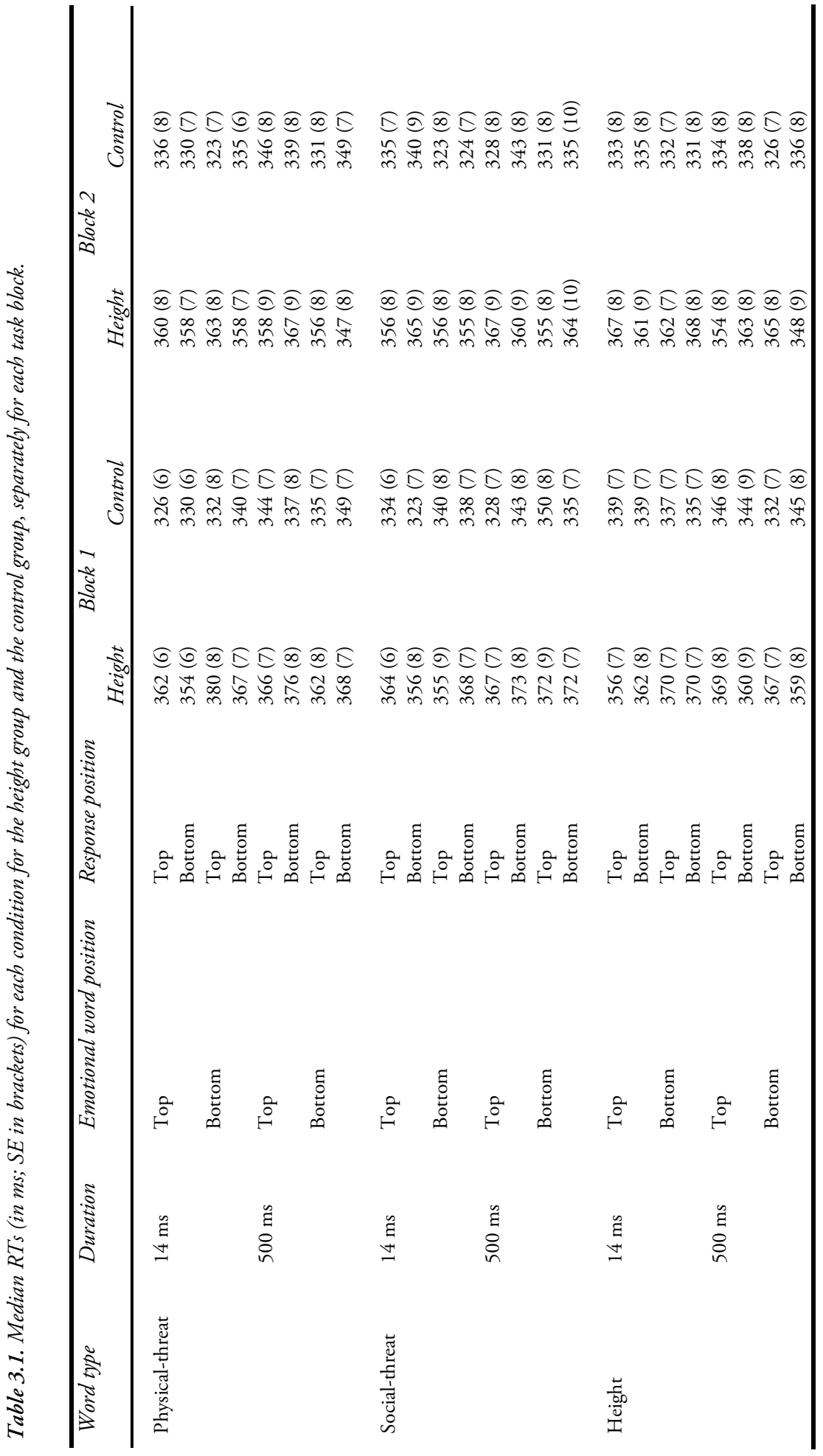




\section{Control group \\ Height group}

Physical-threat words

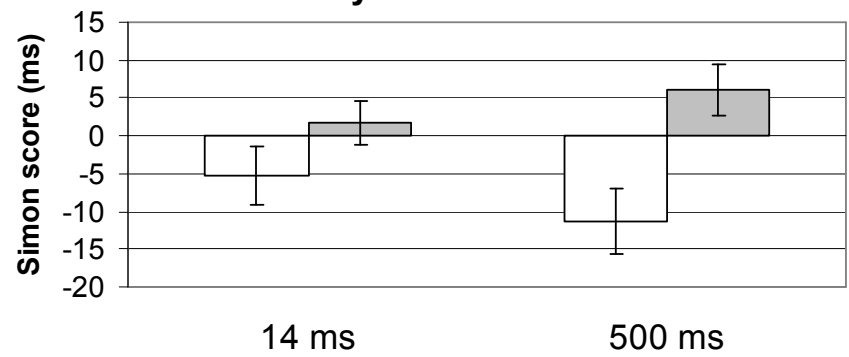

Social-threat words

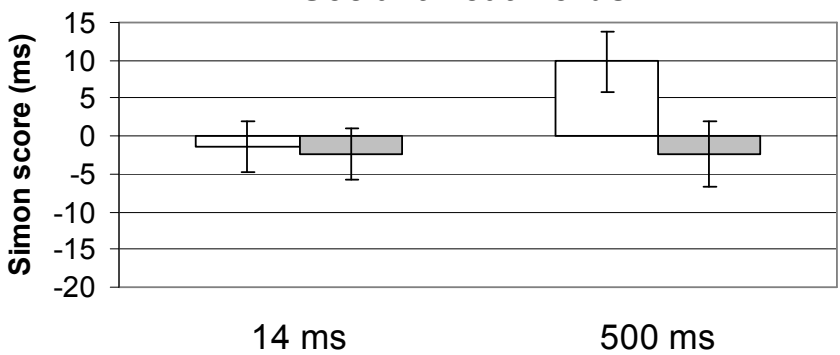

Height words

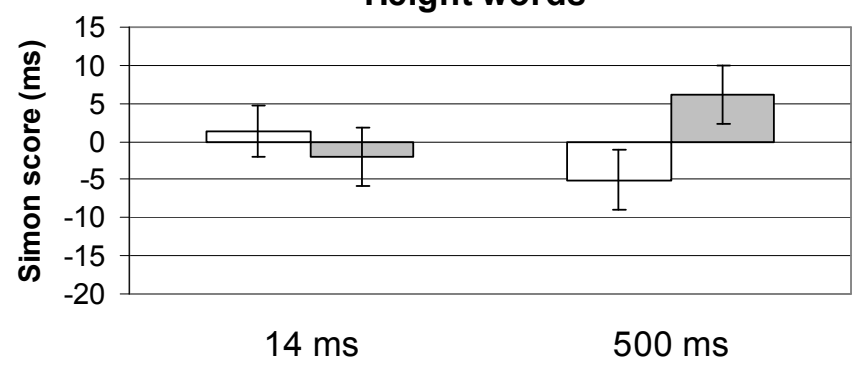

Figure 3.2. Mean Simon scores of the height group and the control group, for physical-threat words, social-threat words and height words for the short $(14 \mathrm{~ms})$ and long $(500 \mathrm{~ms})$ exposure duration, pooled across blocks. Error bars indicate the SE of the group average of Simon scores in each condition, and can be used to judge between-group effects, but not within-subjects effects. 
position $\mathrm{x}$ response position $\mathrm{x}$ group interaction, $F(2,44)=6.5, p=.003$, superseded by duration, $F(2,44)=3.1, p=.06$, and a significant type $\mathrm{x}$ critical word position $\mathrm{x}$ response position $\mathrm{x}$ duration $\mathrm{x}$ block interaction, $F(2,44)=4.4, p=$ .019 .

To simplify these results, Simon scores were calculated by subtracting the mean RT for corresponding critical word and response positions from the mean RT for non-corresponding positions, separately for each type, duration, and block. Positive values reflect the typical Simon effect: faster responses for corresponding than for non-corresponding trials. We hypothesized that the height group would show positive Simon scores for physical-threat words, in both exposure durations, whereas the control group would show no significant effect. We anticipated that there might be a similar pattern of effects for height words, but no significant effects for socialthreat words. Finally, no qualitative differences were expected between the task blocks.

\subsubsection{Simon Effect for Physical-Threat Words}

Figure 3.2 (top panel) suggests for the height group a positive Simon score, and for the control group a negative Simon score for physical-threat words, especially in the $500 \mathrm{~ms}$ condition. The Simon scores for physical-threat words were subjected to an ANOVA with group, duration, and block as factors. There was a highly significant group effect (group: $F(1,45)=10.0, p=.003$ ): for the height group, the positive mean Simon score differed marginally significantly from zero, $t(21)=2.0, p=.056$, and for the control group the negative mean Simon score deviated significantly from zero, $t(24)=-2.6, p=.016$. The group effect was not significantly modified by duration, $F(1,45)=2.1, p=.16$. As can be seen in Figure 3.2 (top panel), a similar pattern of results was obtained in both exposure durations, but the effects were more pronounced in the $500 \mathrm{~ms}$ condition (group: $14 \mathrm{~ms}: F(1,45)=2.0, p=.16 ; 500$ ms: $F(1,45)=9.5, p=.003)$. No significant effects were found for the group $\mathrm{x}$ block interaction, $F(1,45)=1.4, p=.24$, or the group $\mathrm{x}$ duration $\mathrm{x}$ block interaction, $F(1,45)<1 .{ }^{16}$

\subsubsection{Content-Specificity}

Figure 3.2 suggests a similar group difference for height words and physical-threat words, at least in the $500 \mathrm{~ms}$ condition, but the opposite pattern for social-threat

\footnotetext{
${ }^{16}$ A similar pattern of results was obtained when mean RTs were analyzed, except that the group $\mathrm{x}$ block interaction was trend-level significant, $F(1,45)=3.7, p=.06$, suggesting stronger effects in block 2 . Mean RT was based on correct trials within the $150-600 \mathrm{~ms}$ range.
} 
words. Simon scores for physical-threat, social-threat, and height words were entered into a MANOVA with type, duration, block, and group as factors (Pillai's Trace). The type $\mathrm{x}$ group interaction was significant, $F(2,44)=6.5, p=.003$, but was superseded by a trend-level significant type $\mathrm{x}$ group $\mathrm{x}$ duration interaction, $F(2$, 44) $=3.1, p=.06$. The type $\mathrm{x}$ group $\mathrm{x}$ duration interaction can be seen to be due to the strong type $\mathrm{x}$ group interaction in the $500 \mathrm{~ms}$ condition, $F(2,44)=8.9, p=$ .001 , but no such interaction in the $14 \mathrm{~ms}$ condition, $F(2,44)=1.4, p=.26$, demonstrating a high degree of content specificity of Simon effects in the $500 \mathrm{~ms}$ condition. In the $500 \mathrm{~ms}$ condition, the type $\mathrm{x}$ group interaction was significant when contrasting social-threat vs. physical-threat words, $F(1,45)=12.5, p=.001$, or social-threat vs. height words, $F(1,45)=7.4, p=.009$, but not when contrasting physical-threat vs. height words, $F(1,45)<1$. This indicates that the group difference was similar for height words and physical-threat words, but in the opposite direction for social-threat words. No relevant effects were modified by the factor block, all $F(2,44)<1 .{ }^{17}$

A separate analysis on height words revealed that the effects for these words were rather weak: the group effect was only trend-level significant, $F(1,45)=2.9, p$ $=.095$, and neither the height group nor the control group showed a mean Simon score significantly different from zero, $t(21)=1.6, p=.13$, and $t(24)=-.99, p=.33$, respectively. For social-threat words, the group effect was significant, $F(1,45)=4.4$, $p=.04$, and the control group showed a positive Simon score significantly different from zero, $t(24)=2.5, p=.02$ (height group vs. $0: t(21)=-.5, p=.6) .^{18}$

\footnotetext{
${ }^{17}$ The type $\mathrm{x}$ duration $\mathrm{x}$ block interaction reached significance, $F(2,44)=4.4, p=.019$, indicating that only the Simon effects for social-threat words developed over time (duration $\mathrm{x}$ block, $F(1,45)=6.3, p=.016$ ): pooled over groups, the Simon scores for social-threat words presented for $14 \mathrm{~ms}$ increased slightly from the first to the second block, $F(1,45)=3.1, p=.09$, while Simon scores for these words when presented for 500 ms decreased from the first to the second block, $F(1,45)=3.9, p=.06$.

${ }^{18}$ Mean RT analyses (see also Footnote 1) produced equivalent results, except that the type $\mathrm{x}$ group $\mathrm{x}$ duration interaction was no longer (trend-level) significant, $F(2,44)<1$.
} 


\subsection{Discussion}

The present experiment shows for the first time that threat-related words when presented simultaneously with a neutral word can yield a Simon effect. We introduced a task during which one emotional and one neutral word were presented, and the correspondence of emotional stimulus position and response position was manipulated over trials.

In support of our main hypothesis, participants exposed to height responded faster when the position of physical-threat words corresponded to the position of the response than when not. As we argued above, this Simon effect can be taken as a reflection of early preferential processing of physical-threat words. Alternatively, this pattern of responding can also be interpreted in terms of a response tendency to approach stimuli related to physical-threat. Such an approach tendency would be surprising in light of the general view that negative stimuli elicit a withdrawal or avoidance response (Neumann, Förster, \& Strack, 2003). In our paradigm, avoidance would be reflected in relatively slower responses when stimulus and response spatially correspond. It could of course be that the positive Simon score for physicalthreat words in the height group arises from the combination of a positive Simon score caused by early preferential processing and a negative Simon score caused by an avoidance response. The rather unexpected negative Simon score for physicalthreat words in the control group could be explained by a relative dominance of the tendency of all individuals to avoid threat-related stimuli.

A similar response pattern was observed for physical-threat words and height words, although for height words the results did not reach significance. Height words were just like physical-threat words related to the experimental situation of the height group. For social-threat words the opposite pattern of effects was obtained: the control group showed a positive Simon score, while the height group produced a non-significant negative Simon score. In both groups, participants may have had the impression to be evaluated, resulting in a sense of social stress. This social stress might be more pronounced in the control condition than in the height condition where participants were also exposed to height. In that case, in the control condition social-threat words would stand out more than the other words, and therefore be preferentially processed. Taken together, the present findings provide support for content-specific Simon effects.

Simon effects for words presented for $14 \mathrm{~ms}$ were very weak or absent. The daylight on the bridge and the bright lamps in the lab may have reduced the visibility of the stimuli. Further research is required to find out whether threat-related stimuli presented under conditions of restricted awareness can evoke a Simon effect. Just after having viewed the movie scene, the height group felt more tensed than the control group, indicating that our manipulations were successful. We did not 
measure, however, fear of height or physical-threat (trait or state). More research is needed to reveal the influence of these factors.

Finally, De Houwer and Eelen (1998) introduced an affective Simon paradigm during which not the spatial, but the affective correspondence of emotional stimulus and response was manipulated. More specifically, De Houwer and Eelen varied the relation between the affective content of a stimulus (e.g., 'sad' or 'happy') and the valence of the response (e.g., say 'positive' or 'negative'), while neither stimulus nor response had any spatial relevance at all. Hence, the underlying mechanism is probably automatic (involuntary) processing of affective stimulus valence (De Houwer \& Eelen, 1998, p. 48). In our 'spatial' affective Simon task, however, affective stimulus content was presented lateralized. That is, emotional words always appeared on the display opposite a neutral word (one above the other). In addition, we deliberately avoided responses to have an intrinsic affective meaning, and focused solely on the spatial relation between emotional content and response. Therefore, our paradigm is structurally highly different from De Houwer \& Eelen's task, and likely taps an entirely different mechanism.

Taken together, the present study provides new evidence for early preferential processing of threat by showing that in certain conditions threat-related content of words yields a Simon effect. We argue that the present approach offers a promising way to shed light on biased processing of emotional information. 


\section{Chapter 4 \\ Biased Processing of Emotional Information in Anxiety: Evidence from Simon Effects}

A slightly adapted version of this chapter is submitted as: Schrooten, M. G. S., \& Smulders, F. T. Y. (2007). Biased processing of emotional information in anxiety: Evidence from Simon effects. 


\subsection{Abstract}

The present study provides converging evidence on biased processing of threatrelated and positive words in non-clinical anxiety, as revealed by Simon effects. It was hypothesized that when an emotional stimulus is preferentially processed, it may act as if it is presented alone, and have similar effects on responses as a single peripheral stimulus (Simon effects: faster responses when stimulus and response spatially correspond). High- $(n=30)$ and low- $(n=31)$ trait-anxious undergraduates performed a task in which an emotional (physical-threat, social-threat, positive) word was presented simultaneously with a neutral word $(14$ or $500 \mathrm{~ms})$, and spatial correspondence of emotional word and response varied across trials. In the 14-ms condition, high-anxious undergraduates responded faster when social-threat word and response spatially corresponded, whereas they showed the opposite response pattern for positive words. No significant effects were observed in the 500-ms condition. Results are discussed with regard to biased processing of emotional content in anxiety.

\subsection{Introduction}

Over the last twenty years, several cognitive models have been formulated highlighting the role of early preferential processing of threat in anxiety and fear (for a review, see Mogg \& Bradley, 1998). From an evolutionary perspective, our cognitive system would have evolved to automatically detect potential threat, enabling us to react quickly and efficiently if necessary (Oatley \& Johnson-Laird, 1987; Öhman \& Mineka, 2001). This innate system is thought to be especially sensitive in fearful individuals (Öhman, Flykt, \& Esteves, 2001). Models primarily influenced by clinical psychology assume that people differ in how they process threat-related information and that these differences underlie vulnerability to anxiety (Williams, Watts, MacLeod, \& Mathews, 1988; Eysenck, 1997; Mogg \& Bradley, 1998; Mathews \& Mackintosh, 1998).

Empirical support for preferential processing of threat comes largely from studies using emotional Stroop tasks (for a review, see Williams, Mathews, \& MacLeod, 1996), dot-probe tasks (for a review, see Mogg \& Bradley, 1998), and visual search tasks (e.g., Gilboa-Schechtman, Foa \& Amir, 1999; Öhman et al., 2001; Fox et al., 2000). By using these tasks, significant advances have been made in our understanding of cognitive mechanisms involved in anxiety. Notwithstanding their considerable contribution, some inherent difficulties and limitations have been associated with these paradigms. For instance, the interpretation of Stroop and dotprobe effects has been questioned (De Ruiter \& Brosschot, 1994; McKenna \& Sharma, 2004; Fox, 2004), and it is debated whether visual search paradigms can 
reveal any bias for threat in the general population (Tipples, Young, Quinlan, Broks, \& Ellis, 2002).

Recently, we introduced a novel paradigm to investigate early preferential processing of emotional information (Schrooten \& Smulders, 2007a) and used it to examine effects of experimentally-induced physical anxiety. We suggested that if an emotional stimulus presented concurrently with a neutral stimulus is preferentially processed, it may act as if it is presented alone. If so, it was hypothesized, it should stand out of the display and have similar effects on responses as a single stimulus. Single peripheral stimuli yield Simon effects: responses are generally faster if their position corresponds with the position of the response than when not, even if the position of the stimulus is irrelevant to the task at hand (review by Lu \& Proctor, 1995). We suggested that finding a Simon effect for threat-related stimuli presented simultaneously with a neutral stimulus (i.e., faster responses when the position of the threat-related stimulus and response position correspond than when they do not) would add evidence to the hypothesis of preferential processing of threat.

During the task, an emotional and a neutral stimulus were presented simultaneously, and the correspondence of irrelevant emotional stimulus position and response position varied across trials (Schrooten \& Smulders, 2007a). ${ }^{19}$ Participants responded to the color of the stimulus pair. So, any preferential processing was measured as soon as the emotional stimulus appeared. We applied this task in a situation that was potentially physically threatening (exposure to height). As expected, undergraduates exposed to height showed a content-specific Simon effect for physical-threat words. In that study, the groups differed in experimentally induced state-anxiety (exposure versus control), while they were matched for self-reported trait-anxiety.

In the present study, the same paradigm was used to examine early preferential processing of threat-related words in trait-anxiety. It was hypothesized that the highanxious group would show a Simon effect for threat-related words, whereas the lowanxious group would show no Simon effect. Furthermore, these effects might be related to individual's predominant concerns. To examine whether preferential processing of threat is automatic in the sense that it also occurs in conditions of restricted awareness, trials on which masked words were presented for $14 \mathrm{~ms}$ were intermixed with trials with $500 \mathrm{~ms}$ presentation. Contrast-masking studies have

\footnotetext{
${ }^{19}$ De Houwer and Eelen (1998) have also introduced an affective Simon paradigm to investigate automatic affective processing. They manipulated the relation between the task-irrelevant affective stimulus content and the valence of the response, and neither stimulus nor response had any spatial relevance whatsoever. We deliberately avoided responses to have an affective meaning, focusing solely on the spatial dimensions. Our paradigm is thus structurally different from De Houwer \& Eelen's task, and therefore likely measures a different mechanism.
} 
shown that invisible, masked stimuli can affect spatial responses (Vorberg, Mattler, Heinecke, Schmidt, \& Schwarzbach, 2003).

\subsection{Method}

\subsubsection{Participants}

We selected undergraduates reporting high or low levels of trait-anxiety, while displaying low levels of defensiveness. ${ }^{20}$ Trait-anxiety and defensiveness were assessed during pre-screening by the Spielberger State-Trait Anxiety Inventory (STAI-trait; Van der Ploeg, Defares \& Spielberger, 1980) and the Marlowe-Crowne Social Desirability scale (SDS; Hermans, 1967), respectively. Those scoring 42 or more, or 32 or less on the STAI-trait, and scoring 18 or less on the SDS were invited to take part. Thirty-two high-trait-anxious and 32 low-trait-anxious undergraduates were tested. All were native Dutch speakers, dextral, non-dyslexic, and had (corrected to) normal vision and color vision. They received $€ 12.5$ in the form of a gift coupon for their participation. The study was approved by the department's Ethics Committee.

\subsubsection{Materials}

The critical stimuli consisted of 32 social-threat (e.g., failure, insult), 32 physicalthreat (e.g., tumor, injury), and 32 positive (e.g., joy, peaceful) Dutch words, individually matched with neutral words, in terms of length and frequency (CELEX; Baaijen, Piepenbrock, \& Gulikers, 1995). Ninety-six neutral-neutral pairs were created, each pair matched for letter length, to act as fillers. The critical stimuli were drawn from published lists (e.g. Mathews \& MacLeod, 1985); threat-neutral pairs and filler pairs were identical to those used by Schrooten and Smulders (2007a). Word length ranged from three to ten letters.

The affective content of all words was rated by eight staff members ( 3 men; all Dutch) on a visual analogue scale. Physical-threat words, social-threat words, and neutral words paired with them were rated on their association with physical-threat and social-threat, respectively (from 'not at all' to 'extremely'). The valence of positive words, neutral words paired with them, and filler words was rated on a

\footnotetext{
${ }^{20}$ High levels of defensiveness or social desirability may have a confounding effect. It has been suggested that individuals reporting low trait-anxiety yet also high social desirability engage in self-deceptive strategies and direct their attention away from threat-related stimuli, whereas truly low anxious individuals show no bias (Weinberger, Schwartz, \& Davidson, 1979; Fox, 1993a).
} 
bipolar scale (from 'very negative' to 'very positive'). The mean rating difference (zscores) between the members of any physical-threat-neutral pair was at least 1.3 $($ mean $=1.9)$, of any social-threat-neutral pair at least $1.4($ mean $=1.8)$, of any positive-neutral pair at least $1.4($ mean $=1.8)$, of any neutral-neutral pair between .5 and .5 (mean $=.02)$.

During practice trials, additional word pairs were presented. Mirrored, upsidedown upper-case consonants, numbers and symbols were used as backward mask. Masks were unique to each word, length-matched and color-matched with the preceding word, and presented for $14 \mathrm{~ms}$.

\subsubsection{Apparatus}

Task presentation was controlled by a Dell Optiplex GX1 computer, with a Dell P790 17-inch monitor. ERTSVIPL V3.32c (Beringer, 1987) was used to control stimulus presentation and data collection.

\subsubsection{Procedure}

Participants were tested individually, seated at a viewing distance of about $60 \mathrm{~cm}$ from the monitor in a dimly lit room. They were video-monitored and could communicate through a two-way communication system. They were told that the study investigated the relationship between concentration and performance. The real purpose was explained after all participants had been tested.

The design was identical to the one used by Schrooten \& Smulders (2007a), except that positive words instead of height-related words were presented. Figure 4.1 depicts the typical trial configuration. A white central fixation cross was presented for $500 \mathrm{~ms}$. Then, a word pair in upper-case letters (non-proportional font, $0.5 \mathrm{~cm}$ high) appeared on a dark background, with one word above, the other below the center of the screen $(3 \mathrm{~cm}$ apart). In half of the trials, both words were printed in red (Red Green Blue (RGB) color model: 160, 0, 0); in the other half in green (RGB: $0,152,0)$. The cross took on the color of the words. In half of the trials, words appeared for $14 \mathrm{~ms}$, in the other half for $500 \mathrm{~ms}$. In both exposure conditions, words were followed by a mask. 


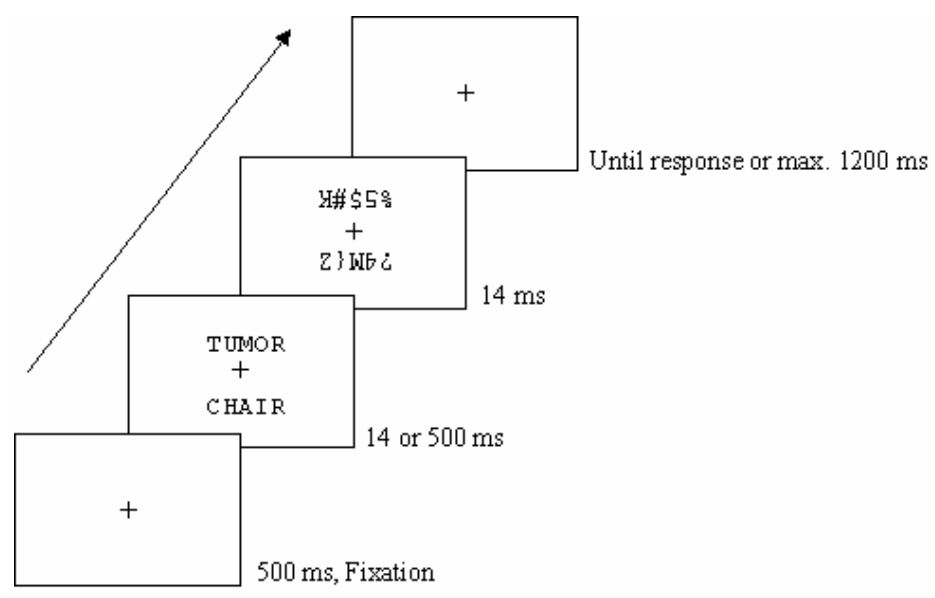

Figure 4.1. Typical trial configuration. The word pair was printed in red or green. The fixation cross took on the color of the word pair and the masks were color-matched with the preceding word pair.

Participants had to respond as quickly and accurately as possible by pressing the top key on a response box with the right index finger to red and the bottom key with the left index finger to green. No instruction was given to process the words in any particular way. Participants had to fixate the cross at all times. After the words disappeared, the cross remained on the screen until the participant responded or for a maximum of $1200 \mathrm{~ms}$. In case of an error, a beep and an error message (showing the instruction) lasting $250 \mathrm{~ms}$ (+ $1500 \mathrm{~ms}$ pause), were inserted. Visual feedback was given if the participant responded too slowly (RT $>800 \mathrm{~ms}$ ) or prematurely (RT $<150 \mathrm{~ms}$ ). The inter-trial interval was $500 \mathrm{~ms}$.

Participants completed two task blocks, each consisting of 36 practice trials, followed by 384 experimental trials. Each block contained an equal number of experimental trials for each combination of type of word pair, exposure duration, position of the critical word and response position. Each word pair was presented once in each exposure duration. Trials were presented in a different random order for each participant. Performance feedback (mean RT) was given every 32 trials during breaks terminated by the participants. 
After the computerized task ${ }^{21}$, the participants completed the STAI-trait, STAIstate, Beck Depression Inventory (BDI; Beck, Ward, Mendelson, Mock, \& Erbaugh, 1961) and SDS. They rated on 9-point scales (ranging from ' $0=$ not at all' to ' $8=$ extremely') how much they recently had been troubled by anxious thoughts about physical health concerns (illness, death, accident) and by anxious thoughts about social concerns (being criticized, being socially evaluated by others; Mogg, Mathews, \& Eysenck, 1992).

\subsection{Results}

\subsubsection{Group Characteristics}

Three participants were excluded, because they had been selected for their high STAI-trait score during pre-screening, but scored below the median STAI-trait score during the experimental session, or they scored relatively low during pre-screening, and above the median during the experiment. The groups differed significantly on all measures except age and gender ratio (see Table 4.1.; men / women ratios for the low- and the high-anxious groups were 5:26 and 7:23, respectively, $\chi^{2}<1, n s$.).

Table 4.1. Group sizes, mean age, and mean scores (SD in brackets) on the STAI Trait and State Anxiety scales, the Beck Depression Inventory (BDI), Marlowe-Crowne Social Desirability Scale (SDS) and 9-point Likert scales, rating social and physical worries.

\begin{tabular}{|c|c|c|c|}
\hline \multirow[b]{2}{*}{ Measure } & \multicolumn{2}{|c|}{ Group } & \multirow[b]{2}{*}{$t(d f=59)$} \\
\hline & $\begin{array}{l}\text { High-anxious } \\
n=30\end{array}$ & $\begin{array}{l}\text { Low-anxious } \\
n=31\end{array}$ & \\
\hline \multicolumn{4}{|l|}{ Age } \\
\hline (range 18-28) & $19.90(2.14)$ & $18.97(.91)$ & 2.23 \\
\hline $\begin{array}{l}\text { Trait-anxiety } \\
\text { (pre-screening) }\end{array}$ & $48.33(5.84)$ & $28.77(2.83)$ & $16.74^{* *}$ \\
\hline $\begin{array}{l}\text { Trait-anxiety } \\
\text { (full-testing) }\end{array}$ & $48.23(6.56)$ & $29.68(5.44)$ & $12.04^{* *}$ \\
\hline State-anxiety & $39.03(8.95)$ & $28.87(5.43)$ & $5.38^{* *}$ \\
\hline BDI & $8.20(7.29)$ & $2.71(1.77)$ & $4.07^{* *}$ \\
\hline SDS & $11.60(4.04)$ & $15.26(4.13)$ & $-3.50^{* *}$ \\
\hline Physical worries & $2.37(1.71)$ & $.77(1.18)$ & $4.25^{* *}$ \\
\hline Social worries & $3.60(1.43)$ & $1.52(1.39)$ & $5.78^{* *}$ \\
\hline
\end{tabular}

Note. STAI - Spielberger State-Trait Anxiety Inventory. Equal variances assumed. * $\mathrm{p}<.01{ }^{* *} \mathrm{p}<.001$

\footnotetext{
${ }^{21}$ During the same session, a probe-classification task was administered, the results of which will be published separately. The presentation order of the tasks was balanced within anxiety groups.
} 


\subsubsection{Behavioral Results}

Incorrect-response trials $(5.1 \%)$ and correct trials with a latency longer than 800 or shorter than $150 \mathrm{~ms}(0.32 \%)$ were eliminated. The groups did not differ in error or outlier rates. Table 4.2. shows mean reaction times (RTs) in each experimental condition.

First, mean RTs were subjected to an ANOVA with critical word position (top, bottom), response position (top, bottom), type (social-threat words, physical-threat words, positive words), duration (14 ms, $500 \mathrm{~ms}$ exposure), and block (first, second task block) as within-subjects factors, and group (high-anxious, low-anxious) as between-subjects factor.

RT was faster in the second block (339 ms) than in the first block (347 ms), $F(1$, $59)=12.4, p=.001$. Faster responses were given by pressing the top key with the right hand to red $(336 \mathrm{~ms})$ than by pressing the bottom key with the left hand to green $(349 \mathrm{~ms}), F(1,59)=33.6, p<.0005$. No other significant main effects emerged.

More relevant are the results involving the interaction between critical word position and response position, reflecting Simon effects. These interactions were summarized in Simon scores, calculated by subtracting the mean RT for corresponding critical word and response positions from the mean RT for noncorresponding positions, separately for each type, duration and block. Positive values reflect the typical Simon effect: faster responses for corresponding than for non-corresponding trials.

Simon scores were entered into an ANOVA with type, duration, block and group as factors. The effects of interest were not further modified by block. Therefore, for each experimental condition, the Simon scores of the blocks were taken together. Figure 4.2 shows the mean Simon scores for each type, duration and group.

As can be seen in Figure 4.2, the high-anxious, compared to the low-anxious group, showed in the 14-ms condition a positive Simon score for social-threat words, and a negative Simon score for positive words, a pattern that reversed in the $500-\mathrm{ms}$ condition. This resulted in a significant type $\mathrm{x}$ duration $\mathrm{x}$ group interaction, $F(1.99,117.68)=8.2, p<.0005$ (Greenhouse-Geissser corrected $d f \mathrm{~s})$. The interaction was significant when contrasting social-threat words vs. positive words, $F(1,59)$ $=15.8, p<.0005$, and social-threat words vs. physical-threat words, $F(1,59)=6.3$, $p=.02$, but not when contrasting physical-threat words vs. positive words, $F(1,59)$ $=2.5, p=.12$. To break down the three-way interaction, separate analyses were conducted for each word type. 


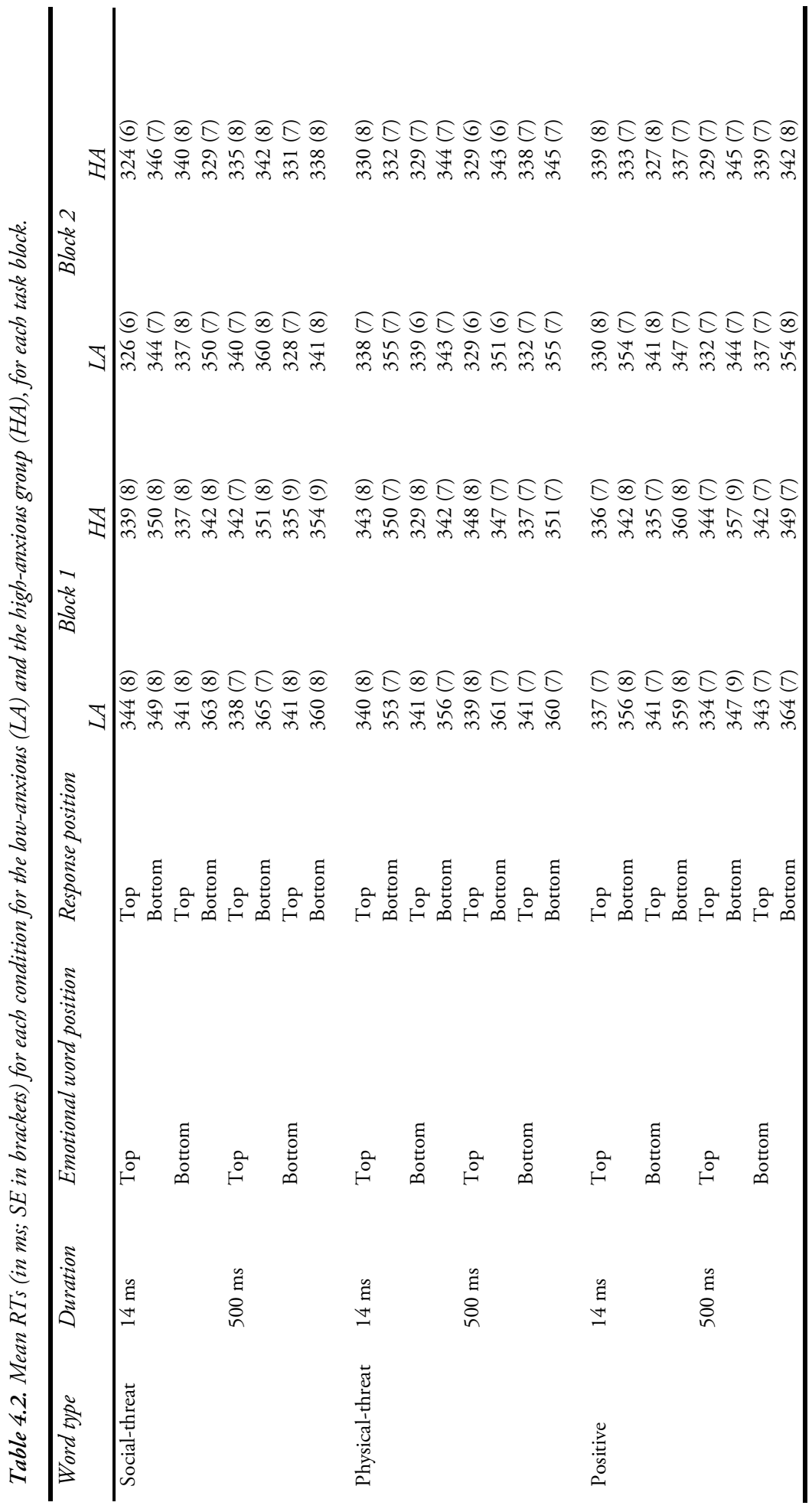




\section{Low-anxious $\quad \square$ High-anxious}
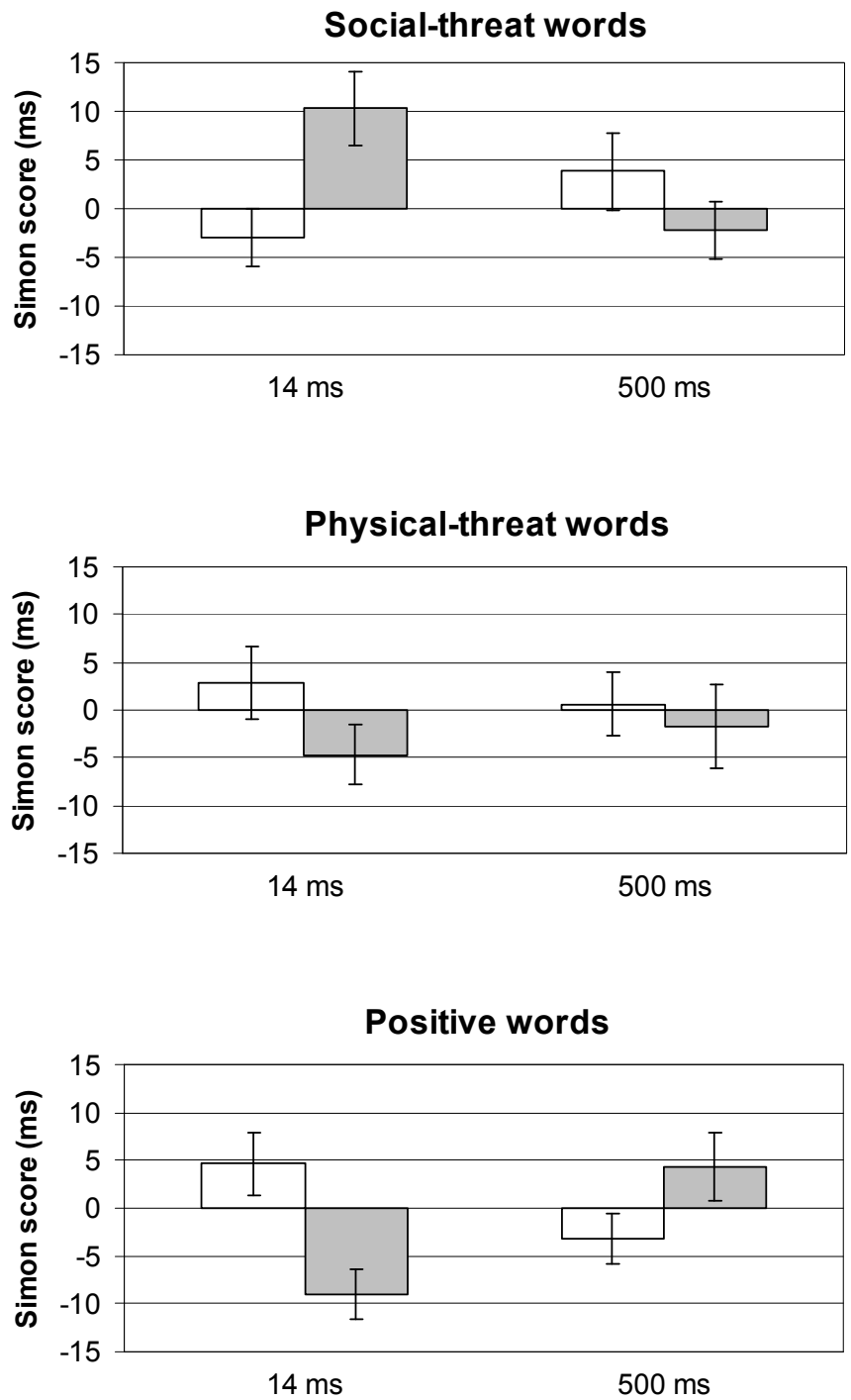

Figure 4.2. Mean Simon scores of the high-anxious and the low-anxious group for social-threat words, physical-threat words, and positive words for the $14 \mathrm{~ms}$ and the $500 \mathrm{~ms}$ exposure duration, pooled across task blocks. Error bars indicate the SE of the Simon scores. 
For social-threat words, there was a duration $\mathrm{x}$ group interaction, $F(1,59)=6.7, p=$ .01. The group effect was significant in the 14-ms condition, $F(1,59)=7.8, p=$ .007 , but not in the $500-\mathrm{ms}$ condition, $F(1,59)=1.4, p=.2$. In the high-anxious group, the Simon score in the 14-ms condition differed from zero, $t(29)=2.7, p=$ .01 . Simon scores obtained during the $500-\mathrm{ms}$ condition and for the low-anxious group did not significantly differ from zero.

For physical-threat words, neither the group effect nor the duration $\mathrm{x}$ group interaction was significant, $F_{\mathrm{S}}(1,59)<1$.

For positive words, there was a duration $\mathrm{x}$ group interaction, $F(1,59)=9.9, p=$ .003. The group effect was significant in the $14-\mathrm{ms}$ condition, $F(1,59)=10.0, p=$ .003 , and trend-level significantly reversed in the 500 -ms condition, $F(1,59)=3.0$, $p=.09$. In the 14-ms condition, the high-anxious group had a negative Simon score for positive words, tested against zero: $t(29)=-3.4, p=.002$. Simon scores obtained during the 500 -ms condition and for the low-anxious group did not differ significantly from zero.

For error rates, Simon scores were calculated in the same way as was done for RTs. An ANOVA of these scores, with type (3), duration, block and group as factors did not reveal a significant group effect, $F(1,59)<1$. Furthermore, there were no significant interactions between group and type and / or duration.

Table 4.3. Partial correlations (two-tailed) between scores on 9-point Likert scales rating social and physical worries, and Simon scores, both for the low-anxious group (LA) and high-anxious group (HA).

\begin{tabular}{|c|c|c|c|c|c|}
\hline & & \multicolumn{2}{|c|}{$\begin{array}{c}\text { Social worries } \\
\text { (controlled for } \\
\text { physical worries) }\end{array}$} & \multicolumn{2}{|c|}{$\begin{array}{l}\text { Physical worries } \\
\text { (controlled for } \\
\text { social worries) }\end{array}$} \\
\hline & & LA & HA & LA & HA \\
\hline \multirow[t]{2}{*}{ Social-threat } & $14 \mathrm{~ms}$ & $.37^{*}$ & .05 & .11 & .19 \\
\hline & $500 \mathrm{~ms}$ & .28 & .04 & $.49^{* *}$ & .30 \\
\hline \multirow[t]{2}{*}{ Physical-threat } & $14 \mathrm{~ms}$ & -.1 & -.05 & -.08 & .21 \\
\hline & $500 \mathrm{~ms}$ & -.09 & .02 & .03 & -.04 \\
\hline \multirow[t]{2}{*}{ Positive } & $14 \mathrm{~ms}$ & .20 & -.31 & .21 & -.17 \\
\hline & $500 \mathrm{~ms}$ & -.22 & .15 & .25 & .04 \\
\hline
\end{tabular}

Note. Because some scores were positive and some negative, correlations were computed in a way that results have positive values when within-group correlations were in the same direction as would be expected based on the corresponding between-groups effect. The same pattern of results was obtained from the zero-order Pearson correlation procedure.

${ }^{*} p<.05 ;{ }^{* *} p<.01$ 


\section{Chapter 4}

To investigate whether the observed effects were related to specific emotional concerns, physical and social worry ratings were correlated to Simon scores based on RTs within each group. Partial correlations are displayed in Table 4.3. Increased social worries within the low-anxious group were associated with more positive Simon scores for social-threat words presented for $14 \mathrm{~ms}$, and increased physical worries in this group were associated with a more negative Simon scores for the same words presented for $500 \mathrm{~ms}$. No other correlations were significant.

\subsection{Discussion}

As expected, high-anxious participants showed a positive Simon score for socialthreat words presented for $14 \mathrm{~ms}$, whereas low-anxious participants had no Simon effect. No significant effects were found for physical-threat words. If positive Simon scores are taken as a reflection of early preferential processing of social-threat words, these findings imply that in the high-anxious group the processing of social-threat words was specifically prioritized. It is not surprising that specifically social-threat words showed this bias, because the STAI-questionnaire that we used for selection is associated to social-threat, rather than physical-threat (Eysenck, 1997). This group difference may have been augmented if our participants, particularly those with a high-anxious personality trait, felt socially evaluated during the experiment. After all, they were video-monitored, and performance feedback was frequently given, both by the experimenter and on the computer screen. In a previous study, we obtained a positive Simon score for physical-threat words, and not for social-threat words, in individuals exposed to a physical stressor (height; Schrooten \& Smulders, 2007a).

The current data also revealed that Simon scores for social-threat words presented for $14 \mathrm{~ms}$ or $500 \mathrm{~ms}$ correlated with social or physical worry ratings, respectively, but only within the low-anxious group. Possibly, the effects for social-threat may reach a ceiling if the level of worrying is higher than some (low) level. As a result, within the continuum from low- to high-anxious, there is a transition from 'no bias' to 'bias' at a relatively low level of anxiety. If so, in the low-anxious group there is sufficient variation in bias scores to find a significant correlation, but in the high-trait-anxious group, there is not, because the whole group has the bias.

The present results indicate that the anxiety-related Simon effects are specific to threat, rather than to emotional content in general. The high-anxious group showed a negative Simon score for positive words presented for $14 \mathrm{~ms}$, whereas the lowanxious group showed no Simon effect. The finding of opposite Simon scores for social-threat and positive words is not that surprising if positive words are considered as opposite in valence to social-threat words. However, the negative Simon scores for positive words are difficult to reconcile with the idea that Simon scores 
purely reflect early preferential processing, because this would imply early preferential processing of the neutral words paired with the positive words. It could be that also approach / avoidance tendencies contribute to the measured Simon scores (Schrooten \& Smulders, 2007a). A tendency to approach stimuli would result in more positive Simon scores and avoidance tendencies in more negative Simon scores. If so, the negative Simon score in the high-anxious group would reflect an avoidance tendency for positive words.

Another possibility is that there is some link between Simon scores and attentional orienting. Several researchers have suggested a close relationship between spatial attention and manual or oculomotor responses (Allport, 1987; Neumann, 1990; Nicoletti \& Umiltà, 1989; Rizzolatti, Riggio, \& Sheliga, 1994). It could be argued that if attention shifts towards a certain stimulus, motor responses in the direction of this stimulus are also prepared, resulting in faster responses with keys spatially corresponding with this stimulus. If a response tendency invariably follows an attentional shift in the same direction, the present pattern of results is effectively explained by supposing for the high-anxious group an attentional bias toward socialthreat words and away from positive words. For both types of attentional bias, there is support in the dot-probe literature (towards social-threat: Bradley, Mogg, Falla, \& Hamilton, 1998; Mansell, Ehlers, Clark, \& Chen, 2002; away from positive: Bradley et al., 1998). Because in our paradigm emotional as well as neutral words carry the relevant information (color), that information is always present at both presumed-attended and -unattended locations and an attentional shift by itself should not lead to faster reactions with one of the response keys. Therefore, attentional bias can only influence Simon scores to the extent that it co-occurs with a genuine response bias.

In the present study, significant effects were only obtained for words presented for $14 \mathrm{~ms}$. Qualitatively different effects were observed for words presented for 500 $\mathrm{ms}$, although with this exposure duration only the group effect for positive words was trend-level significant; the other effects were non-significant. In the 500-ms condition, there was more time for later strategies to come into play. It might be that social-threat and positive words elicit some immediate effect that is later counteracted or even reversed by controlling processes. Several Stroop and dot-probe studies provide evidence suggesting selective processing of subliminally presented threat-related stimuli (e.g, MacLeod \& Rutherford, 1992; Mogg, Bradley, \& Williams, 1995; Mogg \& Bradley, 2002). As in our data, the effects for subliminal stimuli were sometimes stronger (Fox, 2002) (or qualitatively different) than for supraliminal stimuli (MacLeod \& Rutherford, 1992; Mogg, Bradley, \& Hallowell, 1994). As mentioned before, we used the same experimental design to investigate preferential processing of physical-threat words during height exposure (Schrooten \& Smulders, 2007a). In contrast to the present study, significant effects were observed in the 500-ms condition, but no or very weak effects in the 14-ms condition. 


\section{Chapter 4}

This difference in findings between our previous study and the present investigation may be due to the different types of stress or anxiety under investigation. In the previous study, the groups differed in exposure to a physical stressor (height), but were matched for trait-anxiety scores (STAI-trait), whereas in the present study the groups differed in (social) trait-anxiety (STAI-trait). Alternatively, in the previous study the daylight on the bridge and the bright lamps in the lab might have reduced the visibility of the stimuli too far below threshold with 14-ms exposure. In the present study, all participants were tested in a dimly lit room.

In conclusion, the present data provide additional evidence for biased processing of emotional information in non-clinical anxiety. This is the second study showing content-specific Simon effects for threat-relevant words in anxiety. So, the present approach seems to be a useful indirect measurement of biased processing of emotional stimuli. It is apparent that further research is needed to confirm and extend these promising findings. 


\section{Chapter 5 \\ Preferential Processing of Emotional Stimulus Content in Pathological Anxiety: Evidence from Simon Effects}

A slightly adapted version of this chapter is submitted as: Schrooten, M. G. S., Smulders, F. T. Y., \& Arntz, A. (2007). Preferential processing of emotional stimulus content in pathological anxiety: Evidence from Simon effects. 


\subsection{Abstract}

The present study examined biased processing of emotional information in a clinical sample reporting high levels of anxiety. It was hypothesized that when an emotional stimulus is preferentially processed, it may act as if presented alone and elicit a classical Simon effect: faster responses when stimulus position and response position correspond than when not. Eighteen patients with pathological anxiety and 18 healthy controls performed a task during which an emotional (physical-threat, social-threat, positive) word was presented together with a neutral word (14 or 500 $\mathrm{ms}$ ), and spatial correspondence of emotional word and response varied across trials. During the second half of the task, the patient group responded faster when the position of a social-threat or positive word corresponded with response position; there were no significant effects for physical-threat words. The pattern was similar for 14-ms and 500-ms exposure. Results are discussed with regard to preferential processing of emotional stimulus content.

\subsection{Introduction}

The past 20 years have witnessed significant advances in our understanding of the cognitive mechanisms associated with anxiety (e.g., Yiend, 2004a). There is, for example, growing evidence that anxiety is characterized by automatic, preferential processing of threat (e.g., Mogg \& Bradley, 1998; Bar-Haim, Lamy, Pergamin, Bakermans-Kranenburg, \& van IJzendoorn, 2007). Such preferential processing may play a critical role in the maintenance and development of anxiety disorders (e.g., Williams, Watts, MacLeod, \& Mathews, 1988; Eysenck, 1992). Evidence for preferential processing in anxiety comes largely from studies using emotional Stroop tasks (review by Williams, Mathews, \& MacLeod, 1996), dot-probe tasks (review by Mogg \& Bradley, 1998; methodological discussion by Mogg \& Bradley, 1999a) and visual search paradigms (e.g., Gilboa-Schechtman, Foa \& Amir, 1999; Öhman, Flykt, \& Esteves, 2001). Recently, Schrooten and Smulders (2007a, b) provided additional evidence for early preferential processing in sub-clinical anxiety by applying a new approach, described below. In the present study, this approach was followed to examine biased processing in pathological anxiety.

Schrooten and Smulders (2007a) argued that if an emotional stimulus, presented together with a neutral stimulus, is preferentially processed, it may act as if it is actually presented alone. If so, the emotional stimulus should stand out of the display (cf. pop-out in visual search paradigms) and have similar effects on responses as a single stimulus. Single peripheral stimuli typically induce a classical Simon effect: better performance (faster responses, less errors) when stimulus position corresponds with response position than when these positions do not correspond, 
even when stimulus position is irrelevant to the task at hand (review by $\mathrm{Lu} \&$ Proctor, 1995). Simon effects for emotional stimuli presented together with a neutral stimulus are reflected in better performance when emotional stimulus position and response position correspond than when they do not correspond. Finding such an effect would add evidence to the hypothesis of preferential processing of the emotional stimulus, because the stimuli only differ in emotional content.

To study Simon effects for emotional stimuli presented together with a neutral stimulus, Schrooten \& Smulders (2007a) designed a task during which an emotional and a neutral stimulus appeared simultaneously, and correspondence of irrelevant emotional stimulus position and response position systematically varied across trials. Participants responded to the color of the stimulus pair. For example, an emotional and a neutral word were presented one above the other, and participants had to press the top key on a response box when both words were printed in red, the bottom key when both were in green..$^{22}$ In two independent studies employing this task, content-specific Simon effects for threat-relevant words were, under certain conditions, observed for undergraduates. Undergraduates exposed to height responded faster when the position of physical-threat words presented for $500 \mathrm{~ms}$ corresponded to the position of the required response than when not (Schrooten $\&$ Smulders, 2007a). Furthermore, high-trait-anxious students responded faster when social-threat word and response corresponded, and showed the opposite response pattern for positive words, when words were presented for $14 \mathrm{~ms}$; no significant effects were observed with 500-ms (Schrooten \& Smulders, 2007b). The results of these two studies were taken as additional evidence for biased processing of emotional information in non-clinical anxiety. It was concluded that this novel paradigm offers a promising measurement of biased processing of emotional stimulus content.

The present experiment extends our previous research by examining early preferential processing of emotional information in individuals demonstrating clinical levels of anxiety. Patients with pathological anxiety and matched controls performed the novel task, during which physical-threat, social-threat and positive words were presented for 14 or $500 \mathrm{~ms}$. It was hypothesized that the patient group would show content-specific Simon effects for threat-relevant words, whereas the control group would show no (or a significantly smaller) Simon effect. More specifically, it was expected that the patient group would show a Simon effect for social-

\footnotetext{
${ }^{22}$ In another affective variant of the Simon task (De Houwer \& Eelen, 1998), developed to investigate automatic affective processing, correspondence of irrelevant emotional stimulus content and response valence has been manipulated, rather than correspondence of irrelevant emotional stimulus position and response position. Neither stimulus nor response had any spatial relevance at all. Schrooten and Smulders (in press), in contrast, deliberately avoided responses to have an emotional meaning, and focused solely on the spatial dimensions. Since their paradigm is structurally different from previous affective Simon tasks, it likely measures a different mechanism.
} 
threat words, rather than physical-threat words. This expectation was based on our previous finding of specific Simon effects for social-threat words in high-traitanxious undergraduates when tested in a situation where no immediate physical threat was activated.

Given the qualitative differences between clinical and sub-clinical populations, the precise pattern of results of the patient group might differ from the pattern obtained for high-trait-anxious undergraduates. For example, it has been speculated that clinically and sub-clinically anxious individuals, reporting similar high levels of trait anxiety, differ in how well they are able to strategically counteract initial automatic bias effects (Fox, 1996, 2002). This idea was based on the observation that, in Stroop and dot-probe studies, sub-clinically anxious groups often show stronger bias effects for subliminally than for supraliminally presented stimuli, whereas patients show similar bias effects in both conditions. In supraliminal conditions, stimuli were typically presented for a longer duration than in subliminal conditions, and with such longer exposure there is more time for strategic processes to come into play than when stimuli are presented for only a few milliseconds, under restricted awareness. Schrooten and Smulders (2007b) found for high-trait-anxious undergraduates a Simon effect for social-threat words presented for $14 \mathrm{~ms}$ (masked), but not with 500-ms exposure. We expected patients to show Simon effects for emotionally relevant words with 14-ms and 500-ms exposure duration.

\subsection{Method}

\subsubsection{Participants}

Eighteen patients with pathological anxiety participated ( 7 men; mean age 31.7 yrs, $S D=9.6$, range 19.9-48.2). ${ }^{23}$ They were all screened with the Structural Clinical Interview for DSM-IV Axis-I Disorders (SCID-I; First, Spitzer, Gibbon, \& Williams, 1997; Groenestijn, Akkerhuis, Kupka, Schneider, \& Nolen, 1999) and AxisII Disorders (SCID-II; First, Spitzer, Gibbon, Williams, \& Benjamin, 1994; Weertman, Arntz, \& Kerkhofs, 2000) by a qualified clinical psychologist at the Community Mental Health Service center in Maastricht, the Netherlands. Fourteen patients had an anxiety disorder as primary diagnosis: 1 social phobia, 3 panic disorder with agoraphobia ( 2 with additional diagnoses of social phobia and hypo-

\footnotetext{
${ }^{23}$ One additional patient with a primary diagnosis of specific phobia and concurrent depressive disorder was tested, but excluded from further analysis because she reported extremely low scores on anxiety, worrying and depression, compared to the other patients and even to controls.
} 
chondria), 3 panic disorder without agoraphobia (1 with secondary diagnosis of generalized anxiety disorder), 1 agoraphobia without history of panic disorder (additional diagnosis of specific phobia blood-injection-injury), 4 post-traumatic stress disorder, 1 generalized anxiety disorder, and 1 separation anxiety disorder (second diagnosis of panic disorder). Eight of these 14 patients had concurrent depressive disorder. One patient had a first diagnosis of chronic fatigue, a secondary diagnosis of social phobia (not generalized). One patient had a primary diagnosis of somatoform disorder, additional diagnoses of panic disorder with agoraphobia, social phobia in the past, and depressive disorder. Two patients had a primary diagnosis of depressive disorder, a secondary diagnosis of panic disorder or specific phobia (heights). Six patients had an additional axis-2 diagnosis.

Eighteen non-patient controls ( 7 men; mean age $32.2 \mathrm{yrs}, S D=10.8$, range 19.3-50.1) were recruited from the community through flyers and local newspaper advertisements. A first selection of the controls was based on a brief telephone screen. At the end of the experimental session, all controls completed a paper-andpencil version of SCID-I and -II. They had no current medical or psychiatric disorder, had no known history of psychiatric disorders and were medication free. Each control was individually matched, as closely as possible, to a patient in terms of age, gender, and educational level. The groups did not differ in age (paired samples $t(17)=-.7, p=.47)$ and there was a perfect pairwise match by gender. Educational level was low to medium on the average; the patient group contained somewhat more lower schooled individuals than the control group. ${ }^{24}$ Each control reported lower levels of trait-anxiety than the patient with whom he or she was paired; controls scored 41 or less on the trait version of the Spielberger State-Trait Anxiety Inventory (Van der Ploeg, Defares, \& Spielberger, 1980) at the end of the experimental session, whereas patients scored 39 or more (for more details on group characteristics, see Table 5.1.). ${ }^{25}$

All participants were native Dutch speakers, non-dyslexic, and reported (corrected to) normal vision and color vision. They participated on an informed consent basis and received $€ 15$. The study was approved by the ethics committees of University Hospital Maastricht and of Community Mental Health Service Maastricht.

\footnotetext{
${ }^{24}$ Educational level was grouped into 5 categories (lowest level = 1). 1 patient had level 1, 6 patients level 2, 3 patients level 3, no patient level 4, and 8 patients level 5. No control had level 1, 1 control level 2, 5 controls level 3, 2 controls level 4, and 10 controls level 5.

${ }^{25}$ Six controls were tested, but excluded on the grounds that they reported anxiety levels in a clinical range or a history of psychiatric disorder at the end of the session. They were replaced by 6 other controls.
} 


\subsubsection{Materials}

The critical stimuli consisted of 32 social-threat (e.g., foolish, criticism), 32 physical-threat (e.g., disease, fatal), and 32 positive (e.g., pleasant, wise) Dutch words, individually matched with neutral words, in terms of length and frequency (CELEX; Baaijen, Piepenbrock, \& Gulikers, 1995). Ninety-six neutral-neutral pairs were created, each pair matched for length, to act as fillers. Threat-neutral pairs and filler pairs were those used by Schrooten and Smulders (2007a, b); positive-neutral pairs were those used by Schrooten and Smulders (2007b). Word length ranged from three to ten letters. The affective content of all words was rated by eight staff members (3 men; all Dutch) on a visual analogue scale. Physical-threat words, social-threat words, and neutral words paired with them were rated on their association with physical-threat and social-threat, respectively (from 'not at all' to 'extremely'). The valence of positive words, neutral words paired with them, and filler words was rated on a bipolar scale (from 'very negative' to 'very positive'). The mean rating difference ( $z$-scores) between the members of any physical-threat-neutral pair was at least 1.3 (mean = 1.9), of any social-threat-neutral pair at least 1.4 (mean = $1.8)$, of any positive-neutral pair at least 1.4 (mean $=1.8)$, of any neutral-neutral pair between -.5 and .5 (mean $=.02)$.

During practice trials, additional word pairs were presented. Mirrored, upsidedown upper-case consonants, numbers and symbols were used as backward mask. Masks were unique to each word, length- and color-matched with the preceding word, and presented for $14 \mathrm{~ms}$.

\subsubsection{Apparatus}

Task presentation and response registration were controlled by a Dell Latitude D600 laptop, connected to a 17-inch CRT screen, and running ERTSVIPL (Berisoft Cooperation, Frankfurt, Germany).

\subsubsection{Procedure}

Patients were tested just before or in the initial phase of treatment at the Community Mental Health Service center in Maastricht. Patients and controls received the same information before the start of the experiment. They were informed that the study investigated the relationship between concentration and performance. The real purpose was explained at the end of the session. Participants were tested indi- 
vidually, seated at a viewing distance of about $60 \mathrm{~cm}$ from the monitor in a dimly lit room. They were told that they were video-monitored.

The design was identical to the one used by Schrooten and Smulders (2007b), and differed from the one used by Schrooten $\&$ Smulders (2007a) in that positive words instead of height-related words were presented. Figure 5.1 provides an illustration of a typical trial configuration.

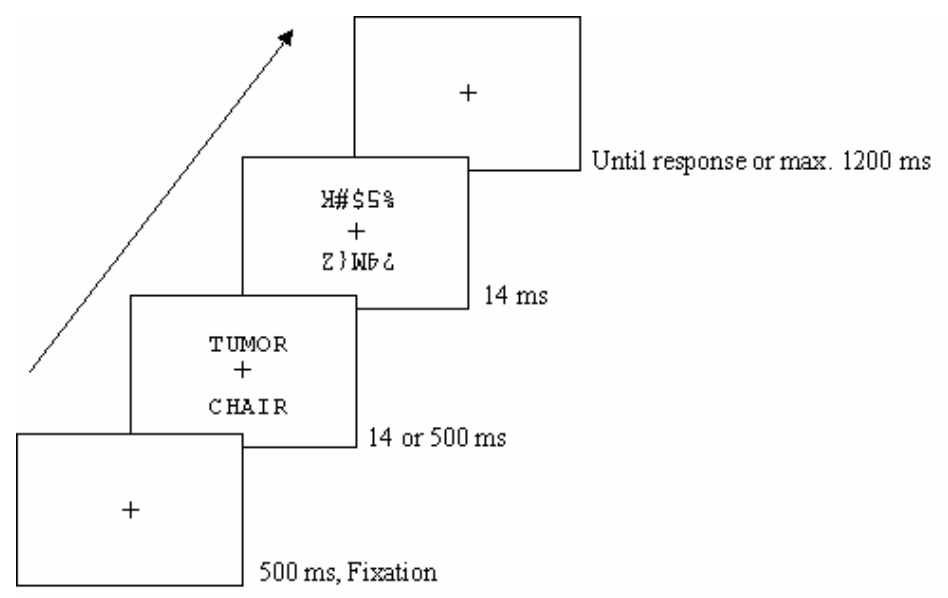

Figure 5.1. Typical trial configuration. The word pair was printed in red or green. The fixation cross took on the color of the word pair and the masks were color-matched with the preceding word pair.

A white central fixation cross was presented for $500 \mathrm{~ms}$. Then, a word pair in upper-case letters (non-proportional font, $0.5 \mathrm{~cm}$ high) appeared on a dark background, with one word above, the other below the center of the screen $(3 \mathrm{~cm}$ apart). In half of the trials, both words (and the fixation cross) were printed in red; in the other half in green. In half of the trials, words appeared for $14 \mathrm{~ms}$, in the other half for $500 \mathrm{~ms}$. In both exposure conditions, words were followed by a mask. Participants had to respond as quickly and accurately as possible by pressing the top key on a response box with the right index finger to red and the bottom key with the left index finger to green. No instruction was given to process the words in any particular way. Participants were encouraged to fixate the cross from its presentation until response. After the words disappeared, the cross remained on the screen until the participant responded or for a maximum of $1200 \mathrm{~ms}$. In case of an error, a beep and 
an error message (showing the instruction) lasting $250 \mathrm{~ms}$ (+ $2500 \mathrm{~ms}$ pause), were inserted. Visual feedback was given if the participant responded too slowly (RT > $2000 \mathrm{~ms}$ ) or prematurely (RT $<150 \mathrm{~ms}$ ). The inter-trial interval was $500 \mathrm{~ms}$.

Participants completed two task blocks, each consisting of 36 practice trials, followed by 384 experimental trials. Each block contained an equal number of experimental trials for each combination of type of word pair, exposure duration, position of the critical word and response position. Each word pair was presented once in each exposure duration. Trials were presented in a different random order for each participant. Performance feedback (mean reaction time) was given every 32 trials during breaks terminated by the participants.

After the computerized task, all participants completed the STAI-trait, STAIstate, Beck Depression Inventory (BDI; measure of depressive state; Beck, Ward, Mendelson, Mock, \& Erbaugh, 1961), Penn State Worry Questionnaire (PSWQ; measure of tendency to worry; van Rijsoort, Vervaeke, \& Emmelkamp, 1997), and Worry Domains Questionnaire Revised (WDQ-R; measure of frequency and content of worrying; van Rijsoort, Emmelkamp, \& Vervaeke, 1999). They rated on 9-point scales (ranging from ' $0=$ not at all' to ' $8=$ extremely') how much they recently had been troubled by anxious thoughts about physical health concerns (illness, death, accident) and by anxious thoughts about social concerns (being criticized, being socially evaluated by others; Mogg, Mathews, \& Eysenck, 1992).

When ready, the control group also completed paper-and-pencil versions of the SCID-I and -II.

\subsection{Results}

\subsubsection{Group Characteristics}

Table 5.1. Mean scores (SD in brackets) on the Spielberger State-Trait Anxiety Inventory (STAI), Beck Depression Inventory (BDI), Penn State Worry Questionnaire (PSWQ), Worry Domains Questionnaire Revised (WDQ-R), and 9-point Likert scales, rating social and physical worries.

\begin{tabular}{llll}
\hline \multirow{2}{*}{ Measure } & \multicolumn{2}{c}{ Group } & \\
\cline { 2 - 3 } & Patient & Control & Paired-samples $t(17)$ \\
& $n=18$ & $32.4(5.4)$ & \\
\hline STAI-trait & $50.4(6.5)$ & $29.3(6.5)$ & $4.6^{*}$ \\
STAI-state & $43.6(9.8)$ & $5.6(4.1)$ & $5.6^{*}$ \\
BDI & $18.8(9.5)$ & $36.8(8.2)$ & $6.4^{*}$ \\
PSWQ & $58.1(12.0)$ & $50.9(14.3)$ & $5.0^{*}$ \\
WDQ-R (total) & $77.5(16.5)$ & $1.4(1.4)$ & $4.3^{*}$ \\
Physical worries & $4.2(2.2)$ & $1.7(1.7)$ & $5.3^{*}$ \\
Social worries & $4.4(1.5)$ & & \\
\hline
\end{tabular}

${ }^{*} p \leq .001$ 
The patient and control group differed in self-reported trait-anxiety, state-anxiety, depression, and worrying. See Table 5.1.

\subsubsection{Behavioral Results}

Incorrect responses ( $4.5 \%$ of all trials) and correct responses with latencies shorter than $150 \mathrm{~ms}$ or longer than $900 \mathrm{~ms}(0.35 \%$ of all correct trials; cutoffs based on visual inspection RT distributions) were eliminated. ${ }^{26}$ There were no significant differences in error or outlier rates between blocks and groups. All reported p values are two-tailed.

Because patients and controls were individually matched and there was a considerable correlation across matched pairs for both median RTs $(r=.45)$ and Simon effects in some conditions $(|r|>.3$; for computation Simon effect, see below), we followed a paired-samples approach in data analysis (cf. randomized-blocks analysis; Howell, 2002, p. 516; Kleinbaum, Kupper, Muller, \& Nizam, 1998). By doing so, possible effects of the matching variables on effects of interest were controlled for.

Median RTs ${ }^{27}$ were first subjected to an overall ANOVA with emotional word position (top, bottom), response position (top, bottom), type (physical-threat words, social-threat words, positive words), duration (14 ms, $500 \mathrm{~ms}$ exposure), block (first, second task block), and group (patient, control) as factors. Table 5.2. shows median RTs in each experimental condition.

Overall, responses were somewhat slower in the patient $(374 \mathrm{~ms})$ than in the control group (345 ms), $F(1,17)=6.7, p=.02$, and faster in the second $(352 \mathrm{~ms})$ than in the first block $(367 \mathrm{~ms}), F(1,17)=15.7, p=.001$. Particularly relevant are the results involving the interaction between emotional word position and response position, reflecting Simon effects. These interactions were summarized in Simon scores, computed by subtracting the mean RT for corresponding emotional word and response positions from the mean RT for non-corresponding positions, separately for each group, type, duration, and block. Positive values reflect a Simon effect for the emotional word: faster responses for corresponding than noncorresponding trials.

\footnotetext{
${ }^{26}$ The results did not change after exclusion of one participant that made comparatively many (12\%) errors and her matched control. The reported results include this pair.

${ }^{27}$ Pattern of results obtained when mean RTs were analyzed was essentially the same as for the median RT results.
} 


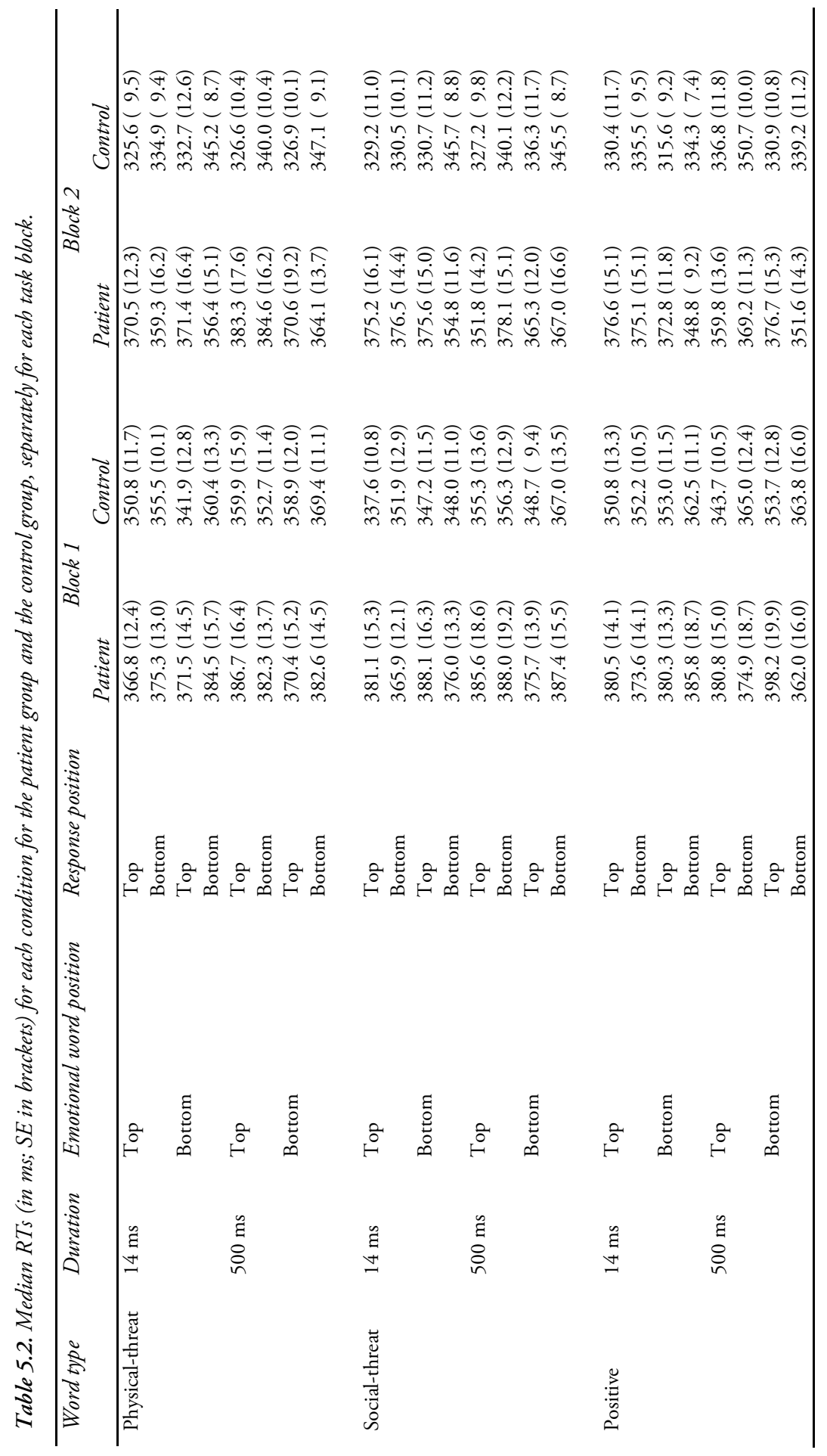


Simon scores were entered into an ANOVA with type, duration, block and group as factors. There was a significant block x group interaction, $F(1,17)=8.6, p$ $=.009$, superseding a main effect of group, $F(1,17)=6.3, p=.02$. In the first block, there were no significant effects, but in the second block, there was a highly significant group effect, $F(1,17)=15.8, p=.001$ : the patient group had a significant positive mean Simon score (9.6; vs. 0 (no bias): $t(17)=4.3, p<.001$ ), whereas the control group had a non-significant negative score $(-2.3$; vs. $0: t(17)=-.7$, ns.). This group effect did not depend on word type or duration, $F_{\mathrm{S}}<1.0$. Figure 5.2 depicts mean Simon scores for the second block, for each group, word type and duration separately.

Visual inspection of Figure 5.2 suggests that Simon effects for social-threat and positive words were similar, and effects for physical-threat words were small or absent. This was confirmed in that a group difference (pooled over durations) was found for social-threat, $t(17)=1.8, p=.08^{28}$ and positive words, $t(17)=3.1, p=$ .007 , but not for physical-threat words, $t(17)=.8, p=.46$. The patient group showed a positive Simon score for social-threat and positive words, tested against zero: $t(17)=2.2, p=.046$, and $t(17)=2.9, p=.009$, respectively (physical-threat: $t(17)=.5, p=.6)$. The control group showed no score significantly different from zero, $p$ s $>.5 .^{29}$

Accuracy data (the log of percentage correct; Schweickert, 1985) were analyzed in the same way as was done for RTs. Analysis of Simon effects revealed a marginally significant effect of group, $F(1,17)=3.3, p=.09$, not further modified by block, type or duration: patients, compared to controls responded more accurately on corresponding than on non-corresponding trials. Importantly, this group effect was in the same direction as the effects observed in RTs, ruling out speed-accuracy trade-off explanations. The group effect was only significant for positive words, $F(1$, $17)=5.0, p=.04$ (for social-threat or physical-threat words, $F_{\mathrm{S}} \leq 1.0$ ).

All 18 patients suffered from clinical levels of anxiety; but for 4 of them, anxiety disorder was only the secondary diagnosis. Excluding them and their matched controls from the analysis did not change the pattern of results as described above.

\footnotetext{
${ }^{28}$ After removal of one outlying participant (score $>$ mean score $+2.5 S D$ ), and her match, the group difference was significant, $t(16)=2.1, p=.049$. The Simon score of the patient group then became $14 \mathrm{~ms}$ (14-ms: 9 ms; 500-ms: $19 \mathrm{~ms}$ ), and the score of the control group -2.5 ms (14-ms: $-5.5 \mathrm{~ms}$; 500-ms: .5 ms).

${ }^{29}$ It might be thought that the group difference in Simon scores was biased by the group difference in overall response speed. However, since the Simon scores of the patient and the control group were opposite in direction, the group difference in overall speed poses no problem for the interpretation of the Simon effects (see also MacLeod, Mathews, \& Tata, 1986, Footnote 1). Anyway, analyses were repeated on individually standardized median RTs (Faust, Balota, Spieler, \& Ferraro, 1999, p. 788). As expected, this transformation removed the group difference in overall speed, but the group differences in Simon effects were unchanged and remained significant.
} 
$\square$ Controls $\square$ Anxious patients
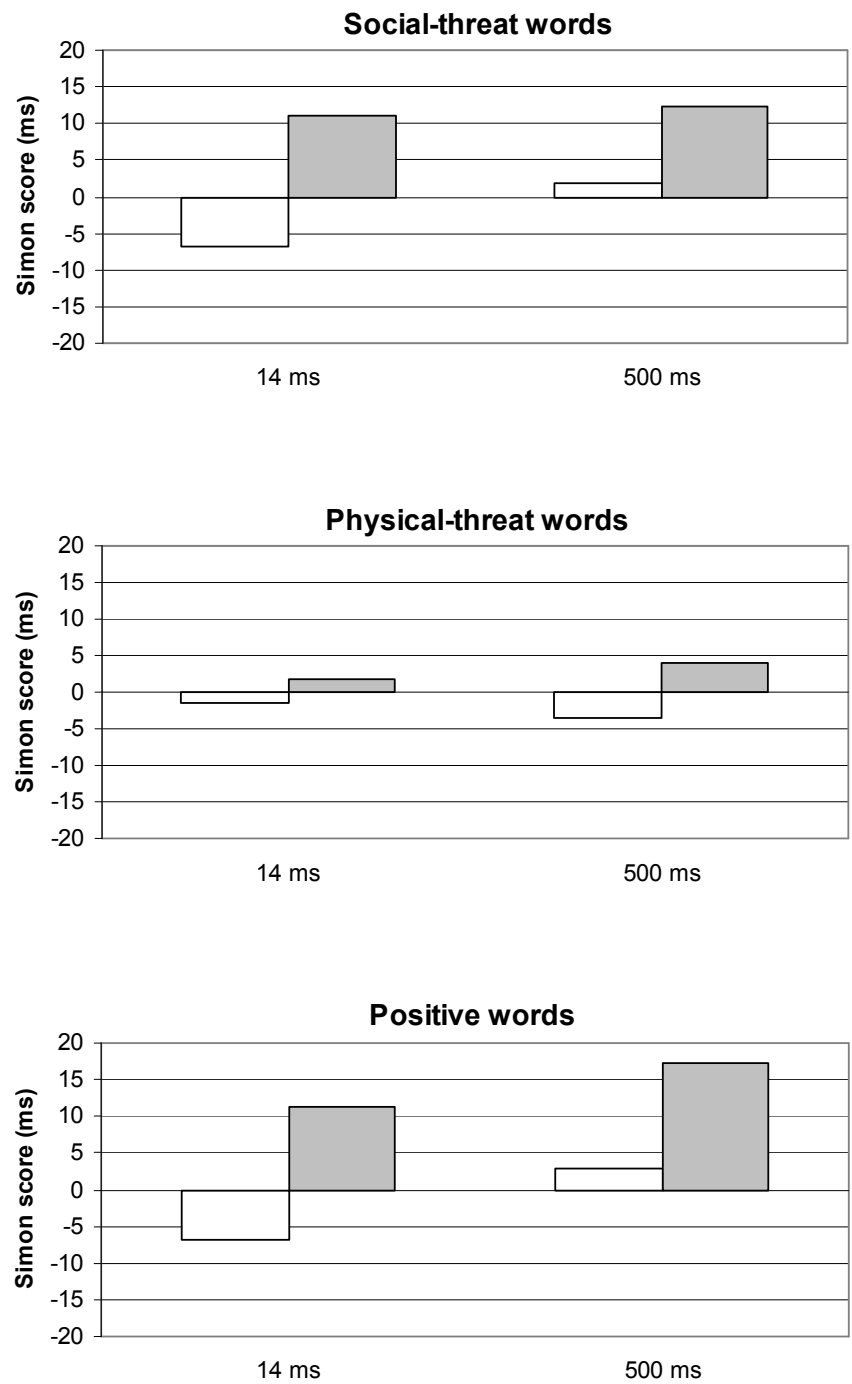

Figure 5.2. Mean Simon scores of the patient group and the control group, for social-threat words, physical-threat words, and positive words for the short $(14 \mathrm{~ms})$ and long $(500 \mathrm{~ms})$ exposure duration during the second task block. 


\subsection{Discussion}

The present findings support the idea of preferential processing of emotional information in a clinical sample reporting high levels of anxiety. The unique contribution of this study lies in demonstrating Simon effects for emotional stimulus content in anxious patients. It extends previous observations of specific Simon effects for emotional stimulus content in undergraduates exposed to a physical stressor (Schrooten \& Smulders, 2007a) and high-trait-anxious undergraduates (Schrooten $\&$ Smulders, 2007b).

Anxiety patients and matched controls performed a task during which an emotional and a neutral word were presented, and the correspondence of emotional word position and response position was systematically varied. During the second half of the task, the patient group revealed a Simon effect for social-threat and positive words, but not for physical-threat words. Response patterns were similar with 14-ms and 500-ms exposure of the words. The control group showed no such bias.

Given the assumption that positive Simon scores in the present task reflect early preferential processing of emotional stimuli, these results suggest that anxious patients are characterized by biased processing of social-threat as well as positive information.

The finding of a Simon effect for social-threat words, but not (or less pronounced) for physical-threat words in anxious patients replicates an earlier finding with high-trait-anxious undergraduates (Schrooten \& Smulders, 2007b). The patients reported higher levels of trait-anxiety than controls on the Spielberger traitanxiety inventory, taken to measure responsiveness to social-threat rather than physical-threat (Fox, 1993a; Eysenck, 1997). Furthermore, social-threat words were clearly more related to the specific test situation, which might have caused social stress, especially in trait-anxious individuals. Participants frequently received performance feedback, by the experimenter (intercom) and on the computer screen, and were repeatedly encouraged to perform as good as possible, while having the impression to be observed (video-monitored). However, the lack of Simon effects for physical-threat words in the patients is also remarkable, as at least 11 patients had anxiety disorders related to physical threat (though they may also have had social worries, notably when agoraphobic). As we found with non-clinical subjects, it might be the case that Simon effects for physical threat only become apparent when physical threat is sufficiently activated (e.g., after forced hyperventilation or $\mathrm{CO} 2$ inhalation).

The response pattern for social-threat words in anxious patients differed, however, in two respects from the previous finding for high-trait-anxious undergraduates: (1) patients showed a positive Simon score with 14-ms as well as with 500-ms 
exposure, whereas undergraduates showed a positive score with 14-ms exposure, but not with 500-ms exposure, (2) in the study with undergraduates, in contrast to the present study, there were no significant differences between task blocks.

The difference in the effect of exposure duration between clinically and subclinically anxious participants was as hypothesized. In previous Stroop- and dotprobe studies, anxious patients often showed similar bias effects with brief and with longer exposure of the stimuli. Sub-clinically anxious individuals on the other hand, often showed a weaker bias with longer than with brief exposure (see Fox, 1996, 2002). Fox (1996, p. 477-478), for example, speculated that an important difference between clinical and non-clinical anxiety lies in that clinically anxious individuals are less able to counteract early processing biases compared to sub-clinically anxious persons. Longer exposure duration, and thus longer processing time, may enable the operation of controlling strategies that take some time to build up. Likewise, the present data suggest that anxious patients preferentially process socialthreat words, even when presented for only $14 \mathrm{~ms}$, and that, because they are unable to control this immediate bias, they still show a bias with $500 \mathrm{~ms}$ exposure.

Explanations for the absence of bias effects in the first block remain speculative at this stage and await further research. Arousal, mood state and / or cognitive processes involved may change over the course of the experiment. It may also be that Simon effects for emotional stimuli only become apparent when performance on the primary task is more automatized. The absence of bias effects in the first block may also be due to the fact the present sample was much lower educated than the formerly investigated student samples, may not use the computer as often, and may not read as automatically as our student sample did. Therefore, it may have taken more time before reading words on a screen was sufficiently automated to let the Simon effects emerge in this clinical sample.

The patient group did show strong Simon effects for positive words. One explanation for positive Simon scores for positive words is that they are due to mood regulating strategies (Bradley, Mogg, White, Groom, \& de Bono, 1999). In line with this idea, in the previous study with undergraduates, the response pattern for positive words was opposite to the pattern for social-threat words (Schrooten \& Smulders, 2007b). With 14-ms exposure, high-anxious undergraduates had a negative Simon score, compared to low-anxious undergraduates; with 500-ms exposure, the group effect was reversed (but small). In the present study, however, similar positive Simon scores were observed for threat-related and positive words, in both exposure durations. It remains to be explained why patients did not apply the supposed mood-regulation strategies to counteract the processing bias for threat words with longer exposure. The finding of anxiety-related bias effects for socialthreat and positive words, but not for physical-threat words, might also be explained by differences in stimulus arousal (e.g., Lang, Bradley, \& Cuthbert, 1997; Russell \& Barrett, 1999). In the present study, the arousal level of the words was not rated. 
Alternatively, similar effects for positive and social-threat words might arise because some of the positive words were related to social evaluation or social situations in general or were antonyms of words related to social threat or anxiety (e.g., applause, self-confident, unconcerned). Possibly these apparently positive words had a threatening meaning for anxious persons and automatically primed their opposites (Mathews \& Klug, 1993). However, close inspection of the data per word suggested that the positive scores for positive words were not exclusively due to this subgroup of positive words.

In the present study, early preferential processing was assessed in pathological anxiety, in general. Patients had various specific diagnoses, but they all had severe levels of anxiety, interfering with daily life. The results of this clinical study extend our earlier work on non-clinical participants reporting high levels of trait anxiety (Schrooten \& Smulders, 2007b). Again, Simon effects for emotional information were observed in an anxious, compared to a non-anxious, sample. An obvious next step is to apply the present promising approach to shed light on the nature of preferential processing associated with specific clinical diagnoses. This was not possible in the present study, because there were not enough patients sharing the same diagnosis.

The finding of positive Simon scores in the present study reflects for the first time the existence of Simon effects (response bias) for social-threat and positive words in anxious patients. The observed Simon effects are interesting for theories on biased processing associated with anxiety. It has been assumed that such Simon effects are due to early preferential processing of the emotional stimulus. That is, an emotional stimulus that is preferentially processed at a very early stage of processing, may pop-out of the display. If so, it may act as if presented alone and elicit a classical Simon effect. However, at this stage, it is not clear which processes exactly underlie these effects. For example, they can also be attributed to enhanced processing of the critical stimulus that does not result in a strict pop-out effect (i.e., attentional bias) or to behavioral tendencies to approach the critical stimulus (Schrooten \& Smulders, in press, 2007b). It is important to note that because in the paradigm used both stimuli convey the relevant color information, an attentional shift by itself should not lead to an increased Simon score. Therefore, attentional bias can only influence the scores to the extent that it occurs together with a response bias.

Taken together, the present data provide additional evidence for biased processing of threat-relevant and positive words in an anxious clinical sample, by applying the task introduced by Schrooten \& Smulders (2007a). The bias effects developed over the course of the task. This task may be a valuable addition to tasks assessing attentional bias, as a different process is assessed: response bias. It may be useful in future research to get an in-depth understanding of cognitive processes associated with emotions and psychiatric disorders. This knowledge may help to improve and evaluate clinical treatment. Furthermore, behavioral paradigms like the one used 
Chapter 5

here may, in the longer term, be applied in diagnosing, predicting therapy success and evaluating patients' progress during therapy. 


\section{Chapter 6 \\ Anxiety-Related Attentional Bias \& Spatial Response Bias Independently Assessed by a Probe-Classification Task}

A slightly adapted version of this chapter is submitted as: Schrooten, M. G. S., \& Smulders, F. T. Y. (2007). Anxiety-related attentional bias and spatial response bias independently assessed by a probe-classification task. 


\section{Chapter 6}

\subsection{Abstract}

Dot-probe tasks have been widely used to investigate attentional bias for threat, reflected in faster responses when threat-related stimulus and probe spatially correspond than when not. The present dot-probe study aimed (a) to investigate whether also spatial correspondence of threat-related word and response influences responses in anxious individuals (spatial response bias), and (b) to examine whether anxietyrelated biases differ as a function of response speed. High- $(n=30)$ and low-traitanxious $(n=31)$ undergraduates performed a probe-classification task in which the correspondences of emotional word, probe and response positions were varied independently. Emotional words (physical-threat, social-threat, positive) were presented with a neutral word for 14 or 500 ms. High-anxious, compared to lowanxious, participants showed (a) a response bias for social-threat words, suggesting preferential processing of these words, (b) an attentional bias towards physical-threat words, and an attentional bias away from social-threat words, irrespective of exposure duration. The group difference in attentional bias for physical-threat words presented for $14 \mathrm{~ms}$ increased with slower responses. This was interpreted in terms of delayed disengagement from threat-related content in anxiety.

\subsection{Introduction}

Considerable research has been devoted to biased information processing in anxiety (for reviews, see Mogg \& Bradley, 1998; Bar-Haim, Lamy, Pergamin, BakermansKranenburg, \& van IJzendoorn, 2007). This research proceeds largely from the idea that preferential processing of threat-related information plays an important role in the maintenance and development of pathological anxiety (e.g., Williams, Watts, MacLeod, \& Mathews, 1988; Eysenck, 1992; Mogg \& Bradley, 1998). A related assumption comes from evolutionary models stating that the adaptive function of anxiety is the automatic detection of threat, so as to react quickly and efficiently when necessary (e.g., Oatley \& Johnson-Laird, 1987).

\subsubsection{Anxiety-Related Attentional Bias}

In investigating selective attention for emotional information in anxiety, the dotprobe paradigm (MacLeod, Mathews, \& Tata, 1986) has been widely applied. The rationale behind this paradigm is that, in general, responses are faster to probes at an attended location than to probes at unattended locations (Posner, Snyder, \& Davidson, 1980). Following the presentation of a stimulus pair, containing two neutral or one neutral and one emotional word or picture, a probe is presented at a location 
previously occupied by one of the members of the pair. Three basic types of the dotprobe task can be distinguished (Mogg and Bradley, 1999a; Salemink, van den Hout, \& Kindt, 2007). In probe-detection tasks, participants press a button on a response box as soon as the probe appears (e.g., MacLeod et al., 1986). In probeposition tasks (e.g., Mogg, Bradley, \& Hallowell, 1994), participants decide during every trial, by pressing one of two buttons, in which of two positions the probe occurred. In probe-classification or -differentiation tasks (e.g., Bradley, Mogg, Falla, \& Hamilton, 1998), participants indicate which type of probe was presented. The size and direction of attentional bias has been calculated by subtracting the reaction time (RT) when emotional stimulus and probe occur in the same position from the RT when they appear in different positions. Positive values reflect a bias towards the emotional stimulus, while negative values reflect a bias away (e.g., Mogg \& Bradley, 1998). However, positive scores may also arise from difficulty in disengaging attention from the location where the emotional stimulus appeared (e.g., Fox, 2004).

In general, probe-detection, probe-position and probe-classification studies report a positive attentional bias score for threat-relevant stimuli, both for anxiety patients (e.g., Mogg, Bradley, \& Williams, 1995; Mogg, Philippot, \& Bradley, 2004) and for individuals with a high-anxious personality trait (e.g., Fox, 1993a; Mansell, Ehlers, Clark, \& Chen, 2002), especially under stress (e.g., MacLeod \& Mathews, 1988; Mogg et al., 1994). A similar bias has been reported for lowanxious people, but only when they were confronted with highly threatening pictures (Mogg, McNamara et al., 2000; Wilson \& MacLeod, 2003). They show either no bias or a negative bias for stimuli with a less threatening content (e.g., MacLeod \& Mathews, 1988; Mogg et al., 1994).$^{30}$ The attentional bias for threat is automatic in that it also occurs for stimuli presented below the (objective) perceptual threshold, i.e. briefly exposed, followed by a mask (e.g., Bradley, Mogg, \& Lee, 1997; Mogg et al., 1994; Mogg \& Bradley, 1999b; 2002). Finally, in general, no bias has been reported in dot-probe experiments for positive stimuli in high-anxious groups, suggesting that the observed bias is not due to a general emotionality effect. Taken together, results from dot-probe experiments have been seen as evidence for an anxiety-related attentional bias for threat-related information.

\subsubsection{Spatial Response Bias}

An originally undervalued aspect of probe-position tasks is that they are sensitive to spatial response bias: the (possible) effects of spatial correspondence of emotional

\footnotetext{
${ }^{30}$ Those reporting low trait-anxiety and also high defensiveness engage in coping strategies and have the tendency to direct their attention away from threat-related information, whereas truly low-anxious individuals show no bias (e.g., Weinberger, Schwartz, \& Davidson, 1979; Eysenck, 1997; Fox, 1993a).
} 


\section{Chapter 6}

stimulus and response. Since in probe-position tasks the spatial position of the probe determines the correct response, there is typically a spatial correspondence of probe and correct response. For example, participants are required to press the lower response key to probes in the lower position and the upper key to probes in the upper position (Mogg et al., 1994). Invariably, when probe and emotional stimulus appear in the same position, the correct response is also spatially corresponding with the emotional stimulus (see Table 6.1.). If the emotional stimulus leads to a tendency to respond towards it, or away from it, this leads to faster responses to the dot, as if it is processed faster. Therefore, attentional bias scores derived from probeposition tasks are $100 \%$ confounded with any response bias. In a similar vein, Fox and collaborators (Fox, Russo, Bowles, \& Dutton, 2001) noted that bias effects observed during a probe-position task, with a single stimulus presented laterally, cannot unambiguously be attributed to attentional processes, but also to motor preparation processes.

Table 6.1. Structure of a typical probe-position task.

\begin{tabular}{|l|l|l|l|l|}
\hline \multicolumn{2}{|c|}{ Probe-position task } \\
\hline \multicolumn{2}{|c|}{ Positions } & \multicolumn{2}{c|}{ Spatial correspondence } \\
\hline \multirow{3}{*}{$\begin{array}{l}\text { Emotional } \\
\text { stimulus }\end{array}$} & Probe & Response & Emotional stimulus - probe & Emotional stimulus - response \\
\hline \multirow{3}{*}{ Top } & Top & Top & Corresponding & Corresponding \\
\cline { 2 - 5 } & Bottom & Bottom & Non-corresponding & Non-corresponding \\
\hline \multirow{2}{*}{ Bottom } & Top & Top & Non-corresponding & Non-corresponding \\
\cline { 2 - 5 } & Bottom & Bottom & Corresponding & Corresponding \\
\hline \multirow{2}{*}{ Non-corresponding minus corresponding $=$} & Attentional bias & Response bias \\
\hline
\end{tabular}


Table 6.2. Structure of a probe-classification task during which all positions vary along a single spatial dimension (i.e., vertically: top/bottom) and during which all positions were varied independently

\begin{tabular}{|c|c|c|c|c|}
\hline \multicolumn{5}{|c|}{ with positions varying along a single spatial dimension } \\
\hline \multicolumn{3}{|c|}{ Positions } & \multicolumn{2}{|c|}{ Spatial correspondence } \\
\hline $\begin{array}{l}\text { Emotional } \\
\text { stimulus }\end{array}$ & Probe & Response & Emotional stimulus - probe & Emotional stimulus - response \\
\hline \multirow{4}{*}{ Top } & \multirow{2}{*}{ Top } & Top & Corresponding & Corresponding \\
\hline & & Bottom & Corresponding & Non-corresponding \\
\hline & \multirow{2}{*}{ Bottom } & Top & Non-corresponding & Corresponding \\
\hline & & Bottom & Non-corresponding & Non-corresponding \\
\hline \multirow{4}{*}{ Bottom } & \multirow{2}{*}{ Top } & Top & Non-corresponding & Non-corresponding \\
\hline & & Bottom & Non-corresponding & Corresponding \\
\hline & & Top & Corresponding & Non-corresponding \\
\hline & & Bottom & Corresponding & Corresponding \\
\hline \multicolumn{3}{|c|}{ Non-corresponding minus corresponding $=$} & Attentional bias & Response bias \\
\hline
\end{tabular}

In contrast to probe-position tasks, in probe-detection tasks and previously published probe-classification tasks there is no systematic spatial correspondence between emotional stimulus and response. In probe-detection tasks the same single button always has to be pressed, and in previous probe-classification tasks spatial correspondence between emotional stimulus and response was avoided. Stimuli and probes were, for example, presented in left and right locations, while responses were given by pressing the upper or lower key (e.g. Bradley et al., 1998; Bradley, Mogg, 


\section{Chapter 6}

White, Groom, \& de Bono, 1999). Suppose now a probe-classification task in which stimulus, probe and response positions vary along a single spatial dimension, for example vertically. That is, words and probes are presented above or below central fixation and responses are given by pressing the upper or lower button. As illustrated in Table 6.2., on only half of the trials where probe and emotional word appear in the same position, responses are spatially corresponding with the position of the emotional stimulus. On the other half, they are non-corresponding. Therefore, attentional bias scores derived from all trials where probe and emotional word are in the same position are not confounded with response bias. The same holds for trials where probe and the emotional word appear in different positions. ${ }^{31}$ This probe-classification task can be used to investigate both attentional bias and response bias.

We argue that response bias is interesting in itself for research on preferential processing of emotional information. Finding a response bias for threat-related stimuli presented simultaneously with a neutral stimulus would add evidence to the hypothesis of early preferential processing of threat. The underlying idea is that, if an emotional stimulus, presented together with a neutral stimulus, is preferentially processed, it may act as if it is actually presented alone. If so, its sudden onset may yield a classical Simon effect: faster responses when emotional stimulus and response spatially correspond than when not (Simon, 1990; Lu \& Proctor, 1995; Ivanoff \& Peters, 2000), that is response bias.

\subsubsection{Response Speed}

In previous dot-probe studies, correct responses have always been collapsed into the mean or the median RT within each condition. In doing this, one assumes that all correct responses, irrespective of response speed, measure exactly the same thing. However, relatively fast responses to the probe occur sooner after emotional stimulus onset and may measure biases at an earlier point in time than relatively slow responses; relatively slow responses may be more affected by processes occurring later in time. Other biases, like for example classical Simon effects, have been shown to depend dramatically on response speed (De Jong, Liang, \& Lauber, 1994; Ridderinkhof, 2002). In the present probe-classification study, RT distributions were analyzed: RTs were divided into quantiles and we examined how attentional bias and response bias effects vary across successive quantiles (cf. De Jong et al., 1994; Ridderinkhof, 2002).

\footnotetext{
${ }^{31}$ In previous probe-classification studies, the orthogonal layout of stimulus and response positions is probably intended to minimize the bias at single trial level. As probe position was irrelevant to the task, any effects of spatial correspondence between emotional word and response would have been balanced, anyway.
} 


\subsubsection{Present Experiment and Predictions}

In the current experiment high-anxious and low-anxious undergraduates performed a probe-classification task in which the spatial correspondence of emotional word and response was balanced over corresponding and non-corresponding emotional word-probe positions, enabling independent assessment of attentional and response bias. Emotional-neutral word pairs (physical-threat, social-threat, positive), probes and responses were vertically arranged and words were presented for $14 \mathrm{~ms}^{32}$ or 500 ms.

We expected that, in line with previous dot-probe results, the high-anxious group would show a content-specific attentional bias for threat-related words, in both exposure conditions, whereas the low-anxious group would show no bias. That is, the high-anxious group would respond faster when threat-relevant word and probe spatially correspond than when they do not. Secondly, it was predicted that the high-anxious group would show a response bias for threat-related words, whereas the low-anxious group would show no bias. That is, the high-anxious group would respond faster when threat-relevant word and response spatially correspond than when they do not. We expected that response bias effects are automatic and occur with threat-relevant stimuli presented for $14 \mathrm{~ms}$. Contrast-masking studies show that stimuli of which participants are not aware can affect spatial responses (e.g., Vorberg, Mattler, Heinecke, Schmidt, \& Schwarzbach, 2003). A similar bias was expected in the $500 \mathrm{~ms}$ condition. Thirdly, to test the hypothesis that anxietyrelated biases would be specific for threat-relevant information and not for emotional stimuli in general, positive words were included. Finally, RT distributions were analyzed to investigate whether attentional bias and response bias effects depend on response speed.

\subsection{Method}

\subsubsection{Participants}

We selected undergraduates reporting extremely high or low levels of trait-anxiety, and low levels of defensiveness. At an initial screening session, trait-anxiety and defensiveness were assessed by the Spielberger State-trait Anxiety Inventory (STAI-

\footnotetext{
${ }^{32}$ Similar exposure durations and masks were used as in previous dot-probe experiments (i.e. word pair as well as mask presented for $14 \mathrm{~ms}$; e.g. Mogg et al, 1994, 1995). In those previous tasks, awareness checks were presented, suggesting that high- and low-anxious participants were unaware of the presence (Mogg et al., 1995) or lexical content (Mogg et al., 1994) of the masked words.
} 


\section{Chapter 6}

trait; Van der Ploeg, Defares \& Spielberger, 1980) and the Marlowe-Crowne Social Desirability scale (SDS; Hermans, 1967), respectively. Those scoring 42 or more, or 32 or less on the STAI-trait, and scoring 18 or less on the SDS were invited to take part. Altogether, 32 high-anxious and 32 low-anxious undergraduates were tested. All were native Dutch speakers, non-dyslectic, right-handed, and reported (corrected to) normal vision and color vision. They received $€ 12.5$. The study was approved by the department's Ethics Committee.

\subsubsection{Materials}

The critical stimuli consisted of 32 social-threat (foolish, lonely), 32 physical-threat (e.g., suffocated, wound), and 32 positive (e.g., joy, cheerful) Dutch words, individually matched with neutral words, in terms of both their length and frequency (CELEX; Baaijen, Piepenbrock, \& Gulikers, 1995). Ninety-six neutral-neutral pairs were created, each pair matched for letter length, to act as fillers. The critical stimuli were mostly drawn from published lists (e.g. MacLeod et al., 1986). Word length ranged from three to ten letters.

The affective content of all words was rated by eight staff members ( 3 men; all Dutch) on a visual analogue scale. Physical-threat words, social-threat words, and neutral words paired with them were rated on their association with physical-threat and social-threat, respectively (from 'not at all' to 'extremely'). The valence of positive words, neutral words paired with them, and filler words was rated on a bipolar scale (from 'very negative' to 'very positive'). The mean rating difference ( $z$ scores) between the members of any physical-threat-neutral pair was at least 1.3 $($ mean $=1.9)$, of any social-threat-neutral pair at least $1.4($ mean $=1.8)$, of any positive-neutral pair at least $1.4($ mean $=1.8)$ and of any neutral-neutral pair between -.5 and .5 (mean $=.02)$.

During practice trials, additional word pairs were presented. White, mirrored, upside-down upper-case consonants, numbers and symbols were used as backward mask. Masks were unique to each word, length-matched with the preceding word, and presented for $14 \mathrm{~ms}$.

\subsubsection{Experimental Hardware}

Task presentation was controlled by a Dell Optiplex GX1 computer running ERTSVIPL V3.32c (Beringer, 1987). 


\subsubsection{Procedure}

Participants were tested individually, seated at a viewing distance of $60 \mathrm{~cm}$ from the monitor in a dimly lit testing room. They were video-monitored and could communicate through a two-way communication system. They were told that the study investigated the relationship between concentration and performance. The real purpose of the experiment was explained after all participants had been tested.

Each trial started with the presentation of a white central fixation cross for 500 ms. A backwardly masked word pair in white upper case letters (non-proportional font, approximately $0.5 \mathrm{~cm}$ high) appeared on a dark background, with one word above, the other below the center of the screen $(3 \mathrm{~cm}$ apart). In half of the trials, words appeared for $14 \mathrm{~ms}$, in the other half for $500 \mathrm{~ms}$. Immediately following the display of the mask, a small dot $(3 \times 2 \mathrm{~mm})$ was presented at the location previously occupied by one of the words. In half of the trials the dot was red, in the other half green. All participants had to respond as quickly and accurately as possible by pressing the top key with the right index finger to red and the bottom key with the left index finger to green. No instruction was given to process the words in any particular way. Participants were encouraged to fixate the cross from its presentation until response execution. The probe was displayed until a response was made or $1200 \mathrm{~ms}$ had elapsed. The inter-trial interval was $500 \mathrm{~ms}$. In case of an error, a beep and an error message (showing the instruction) lasting $250 \mathrm{~ms}$ (+ $1500 \mathrm{~ms}$ pause), were inserted. Visual feedback was given if the participant responded too slowly (RT $>800 \mathrm{~ms}$ ) or prematurely (RT $<150 \mathrm{~ms}$ ).

All participants completed two task blocks, each consisting of 36 practice trials, followed by 384 experimental trials. Per block, there were an equal number of experimental trials for each combination of type of word pair, exposure duration, position of the critical word, probe position, and response position. Each word pair was presented once in each exposure duration. The experimental trials were presented in a different random order for each participant. Performance feedback (mean RT) was given every 32 trials during breaks terminated by the participants.

After the computerized task ${ }^{33}$, the participants completed Dutch versions the STAI-trait, STAI-state, Beck Depression Inventory (BDI; Beck, Ward, Mendelson, Mock, \& Erbaugh, 1961) and SDS.

\footnotetext{
${ }^{33}$ In the same experimental session, an affective variant of the Simon task was administered, the results of which will be published separately. The presentation order of the tasks was balanced within anxiety groups.
} 


\subsection{Results}

\subsubsection{Group Characteristics}

Three participants were excluded from the analyses, because they had been selected for their high STAI-trait score during pre-screening, but scored below the median STAI-trait score during the experimental session, or they scored low during prescreening, and above the median during the experiment. The groups differed significantly on all measures except for age and gender ratio (see Table 6.3.; men / women ratios for the low- and the high-anxiety groups were 5:26 and 7:23, respectively, $\left.\chi^{2}<1\right)$.

Table 6.3. Group sizes, mean age, and mean scores (SD in brackets) on the STAI Trait and State Anxiety scales, the Beck Depression Inventory (BDI), and Marlowe-Crown Social Desirability Scale (SDS).

\begin{tabular}{llcc}
\hline \multirow{2}{*}{ Measure } & \multicolumn{2}{c}{ Group } & \multirow{2}{*}{$\begin{array}{l}\text { Low-anxious } \\
n=31\end{array}$} \\
\cline { 2 - 3 } & $\begin{array}{l}\text { High-anxious } \\
n=30\end{array}$ & $18.97(.91)$ & 2.23 \\
\hline $\begin{array}{l}\text { Age } \\
\text { (range 18-28) }\end{array}$ & $19.90(2.14)$ & $28.77(2.83)$ & $16.74^{* *}$ \\
$\begin{array}{l}\text { Trait-anxiety } \\
\text { (pre-screen) }\end{array}$ & $48.33(5.84)$ & $29.68(5.44)$ & $12.04^{* *}$ \\
$\begin{array}{l}\text { Trait-anxiety } \\
\text { (experiment) }\end{array}$ & $48.23(6.56)$ & & \\
State-anxiety & $39.03(8.95)$ & $28.87(5.43)$ & $5.38^{* *}$ \\
BDI & $8.20(7.29)$ & $2.71(1.77)$ & $4.07^{* *}$ \\
SDS & $11.60(4.04)$ & $15.26(4.13)$ & $-3.50^{* *}$ \\
\hline
\end{tabular}

Note. STAI - Spielberger State-Trait Anxiety Inventory. Equal variances assumed.

${ }^{*} p<.01{ }^{* *} p<.001$

\subsubsection{Behavioral Results}

First, incorrect responses (5.24 \% of all trials) were excluded from the database. The RTs of the remaining trials were used for distributional analyses. For each participant, block and condition, five $20 \%$ RT quantiles were made according to Ratcliff (1979) and averaged across participants within each anxiety group. For the analysis of mean RT, responses with latencies longer than 1000 or shorter than $150 \mathrm{~ms}(0.1$ $\%$ of all correct trials) were excluded. The anxiety groups did not differ significantly in error or outlier rates. Unless stated otherwise, for $F$-values $d f$ s were $(1,59)$ and for $t$-values $d f$ s were (59). All reported $p$-values are two-tailed. 
Mean RTs were entered into an overall ANOVA with type (physical-threat, social-threat, positive), duration (14 ms, $500 \mathrm{~ms}$ exposure), emotional word position (top, bottom), probe position (top, bottom), response position (top, bottom), block (first, second task block) and group (high-anxiety, low-anxiety) as factors. RTs were in general shorter in the second $(466 \mathrm{~ms})$ than in the first block $(480 \mathrm{~ms} ; F=34.4$, $p<.0001)$, when the top key was pressed ( $465 \mathrm{~ms})$ than when the bottom key was pressed (481 ms; $F=60.7, p<.0001$ ), to probes on the top ( $471 \mathrm{~ms}$ ) than to probes on the bottom position ( $475 \mathrm{~ms} ; F=4.2, p=.045$ ), and in the $500 \mathrm{~ms}$ exposure condition (459 ms) than in the $14 \mathrm{~ms}$ exposure condition (487 ms; $F=111.1, p<$ $.0001)$.

Attentional bias and response bias are discussed successively. For both, first mean RTs were analyzed, and then distributional analyses were carried out. The results section focuses on RT analyses, for no significant anxiety-related bias effects were observed for error rates.

\subsubsection{Attentional Bias}

\subsection{Mean Attentional Bias}

Attentional bias scores were computed by subtracting the mean RT when the probe appeared on the same position as the preceding emotional word from the mean RT when the probe and the emotional word appeared in different locations. These scores were subjected to an ANOVA with type, duration, block, and group as factors. As can be seen in Figure 6.1a, attentional bias effects strongly depended on word type (type $\mathrm{x}$ group, $F(1.85,109.19)=5.3, p=.008$, Greenhouse-Geisser corrected $d f s$ ). Figures $6.1 \mathrm{~b}$ and $6.1 \mathrm{c}$ display the attentional bias scores in the first and second block, respectively. For each word type separately, attentional bias scores were entered into an ANOVA with block, duration and group as factors.

Physical-threat. For physical-threat words, a significant group effect was found, $F=6.0, p=.017$, not further modified by block, duration or their interaction, $F$ s $<$ 1.0. One sample t-tests comparing the bias scores (pooled across durations and blocks) with zero (no bias) revealed for the high-anxious group a positive attentional bias for physical-threat words, $t(29)=2.1, p=.04$, while the low-anxious group showed no significant bias, $t(30)=-1.29, p=.21$. No significant block effect was revealed. However, Figure 6.1c suggests that for physical-threat words presented for $14 \mathrm{~ms}$, there was only an effect in block 1, not in block 2 . This observation was confirmed by distributional analyses (see below).

Social-threat. For social-threat words, attentional bias tended to be more negative for high-anxious than for low-anxious participants (group, $F=3.3, p=.07$ ), especially in block 1 (group x block, $F=2.8, p=.1$ ). Separate analyses per block indeed revealed a significant group effect in block $1, F=5.1, p=.03$, but not in 


\section{Chapter 6}

block $2, F<1.0$. In neither block, there were effects of duration (block 1: duration: $F=2.1, p=.15$, duration x group: $F<1.0$; block 2 : duration: $F=1.6, p=.22$, duration x group: $F<1.0){ }^{34}$ One-sample t-tests against zero in block 1 revealed a marginally significant negative bias in the high-anxious group, and no effect in the low-anxious group, $t(29)=-1.85, p=.075, t(30)=1.35, p=.19$, respectively. ${ }^{35}$

Positive. There was a small group difference for positive words presented for 500 $\mathrm{ms}$ in block 2 (block $\mathrm{x}$ duration $\mathrm{x}$ group, $F=2.5, p=.10$ ). The duration $\mathrm{x}$ group interaction was close to significance in block $2, F=3.7, p=.06$, while no significant effects of group and duration were found in block $1, F_{\mathrm{s}}<1$. Furthermore, in block 2 there was a weak group effect in the $500 \mathrm{~ms}$ condition, $F=2.9, p=.09$, but not in the $14 \mathrm{~ms}$ condition, $F=1.1, p=.29$. Tested against zero, the positive bias shown by the high-anxious group for positive words presented for $500 \mathrm{~ms}$ in block 2 was marginally significant, $t(29)=1.6, p=.1$. No other bias effects differed from zero.

In sum, the high-anxious group, compared to the low-anxious group, showed a positive attentional bias for physical-threat words. In addition, a (weak) negative attentional bias was observed for social-threat words in block 1 and a weak positive bias in the high-anxious group for positive words presented for $500 \mathrm{~ms}$ in block 2 . One reason for effects being only trend-level significant for mean RT may be that they are limited to a specific portion on the distribution.

\subsection{Distributional Analyses Attentional Bias}

For each quantile, attentional bias scores were computed as described above. To assess how the attentional bias effects vary across successive quantiles, polynomial trend analyses (linear and quadratic) were executed. That is, for each word type separately, bin (five quantiles from the fastest to the slowest) was included as a polynomial contrast in an ANOVA with duration, block, and group as factors, and attentional bias as dependent variable.

\footnotetext{
${ }^{34}$ After removal of an outlier in attentional bias scores for social-threat words in the $14 \mathrm{~ms}$ condition in block 1 , the main effect of duration was significant, $F(1,58)=4.3, p=.04$, indicating more positive bias scores in the $500 \mathrm{~ms}$ condition. This duration effect was still not further modified by group, $F<1.0$. The group effect remained significant $F(1,58)=4.0, p=.049$.

${ }^{35}$ When the data were broken down by duration nevertheless, the high-anxious group showed in block 1 a negative bias in the $14 \mathrm{~ms}$ condition significantly different from zero, $t(29)=-2.3, p=.03$, but no bias in the $500 \mathrm{~ms}$ condition, $t(29)=-.9, p=.37$. The low-anxious group, in contrast, showed a positive bias in the 500 ms condition marginally significantly different from zero, $t(30)=1.98, p=.06$, but no bias in the $14 \mathrm{~ms}$ condition, $t(30)=.06, p=.95$.
} 
A. Pooled across blocks

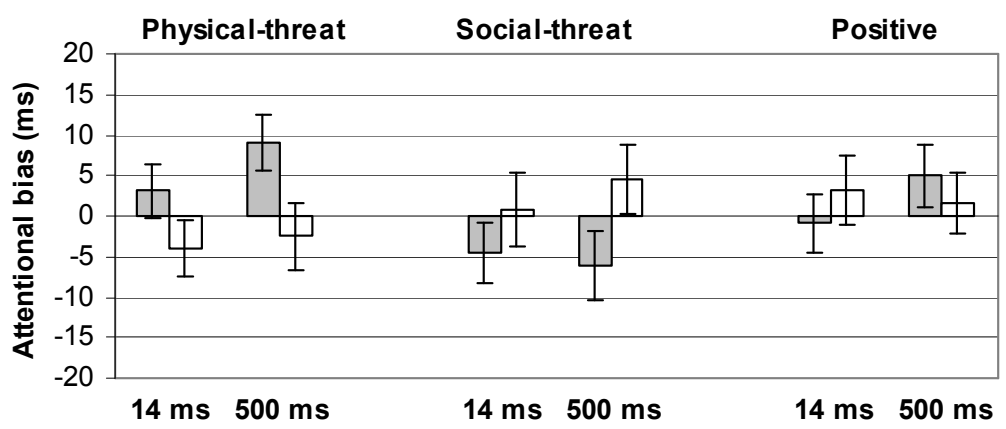

B. Block 1

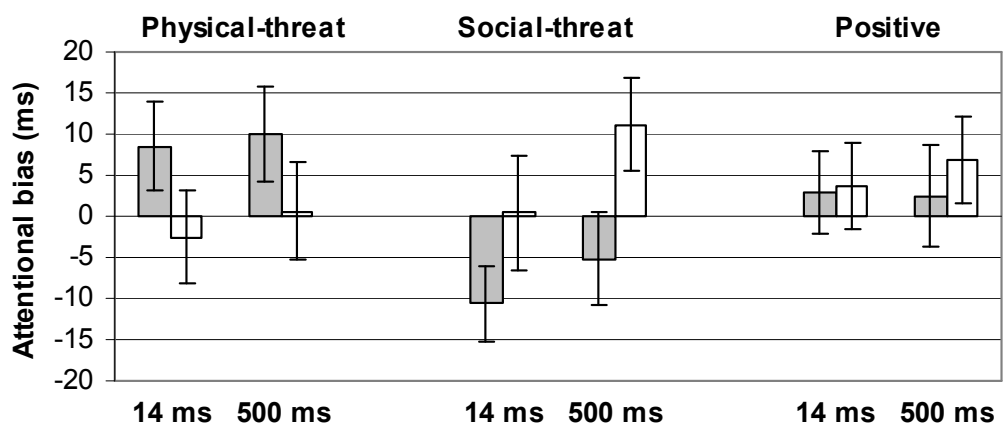

C. Block 2

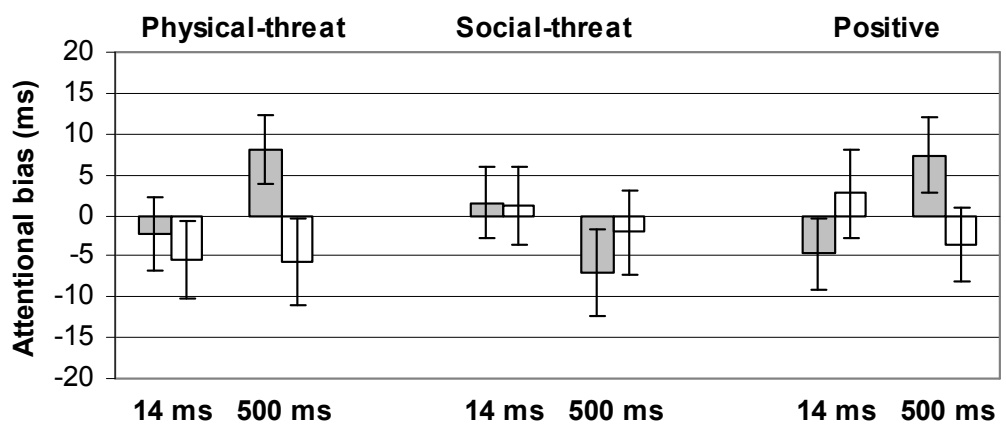

Figure 6.1. Mean attentional bias scores of the high-anxious and the low-anxious group for physicalthreat words, social-threat words, and positive words for the $14 \mathrm{~ms}$ and the $500 \mathrm{~ms}$ exposure duration, pooled across task blocks (a) and for the two task blocks separately ( $b$ and $c$ ). 


\section{Chapter 6}

Physical-threat. Figures 6.2a-d show the attentional bias scores for physicalthreat words per bin for each group, duration and block separately. ${ }^{36}$ Figure 6.2 suggests that the group difference for physical-threat words presented for $14 \mathrm{~ms}$ increased with slower RTs in block 1 (2a), that there was no group difference for these words in block 2 (2c), and that the group difference for these words in the 500 ms condition did not depend on RT, in either block $1(2 \mathrm{~b})$, or 2 (2d). Statistical analyses revealed that the group effect for physical-threat words increased across successive bins (group $\mathrm{x}$ bin-linear, $F=3.6, p=.06$ ). A significant block $\mathrm{x}$ duration $\mathrm{x}$ group $\mathrm{x}$ bin-quadratic interaction was found, $F=5.4, p=.02$. Furthermore, for physical-threat words presented for $14 \mathrm{~ms}$, the block $\mathrm{x}$ group $\mathrm{x}$ bin-quadratic interaction was nearly significant, $F=3.8, p=.055$. In block 1 (Figure 6.2a), the group effect for these words increased gradually across successive bins (group $\mathrm{x}$ bin-linear, $F=3.8, p=.06$ ), especially from the fourth to the fifth bin (group $\mathrm{x}$ bin-quadratic, $F=4.4, p=.04)$. Whereas the positive bias shown by the high-anxious group remained stable across bins, $F(1,29) \mathrm{s}<1.0$, the negative bias of the low-anxious group increased with slower RTs (bin-linear, $F(1,30)=4.1, p=.05$ ), especially from the fourth to the fifth bin (bin-quadratic, $F(1,30)=3.6, p=.07$ ). In block 2 (Figure 6.2c), no significant (bin) effects were observed, $F_{\mathrm{s}}<1.0$.

For physical-threat words presented for $500 \mathrm{~ms}$, there were no significant effects for the bin $\mathrm{x}$ group interaction (linear, $F<1.0$; quadratic, $F=1.5, p=.23$ ), or for the bin $\mathrm{x}$ group $\mathrm{x}$ block interaction (linear, $F<1.0$; quadratic, $F=1.2$, $p=.27$ ).

Social-threat. The group effect for social-threat words did not vary across bins (group x bin-linear / quadratic, $F_{\mathrm{S}}<1.0$; not modified by duration and / or block).

Positive. For positive words, there were no significant bin effects, $F_{s}<1.0$.

In sum, there was an anxiety-related positive bias for physical-threat words presented for $14 \mathrm{~ms}$, but only in block 1 . The group difference for this bias strongly increased with slower responses. This effect was hidden in the mean RT analyses. In addition, there was an anxiety-related positive bias for physical-threat words presented for $500 \mathrm{~ms}$, that did not depend on block or RT. Altogether, these findings suggest that only the combination of a short exposure duration and relatively fast RTs would not yield an attentional bias for physical-threat words in block 1. Rather weak attentional bias effects were found for social-threat and positive words. These effects did not vary as a function of response speed.

\footnotetext{
${ }^{36}$ In each bin, the groups did not significantly differ in mean RT, $.001 \leq F_{\mathrm{S}} \leq 2.2, .14 \leq p s \leq 9.8$. Mean bin RTs are presented in Figure 2 (in brackets).
} 

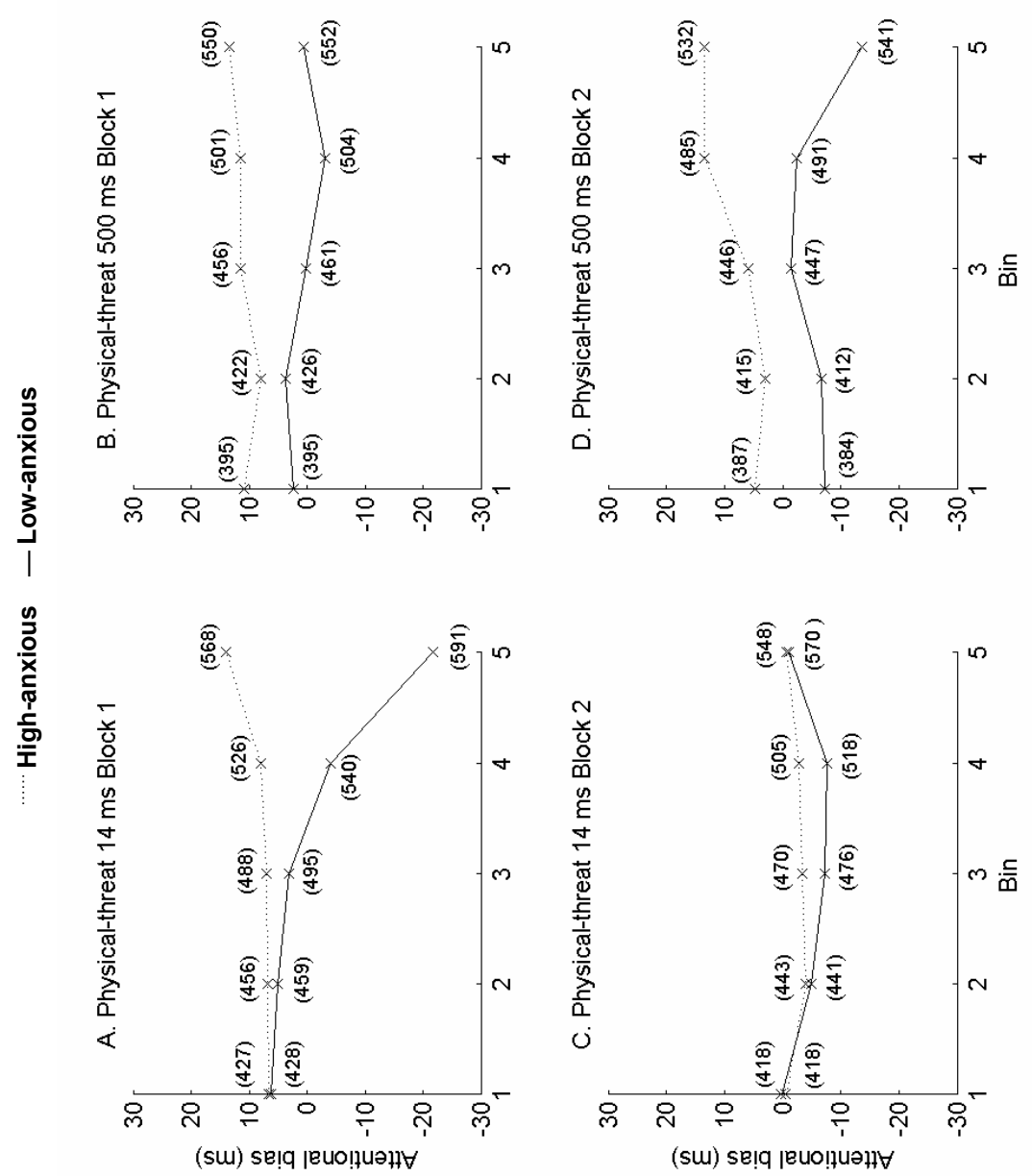

+क.

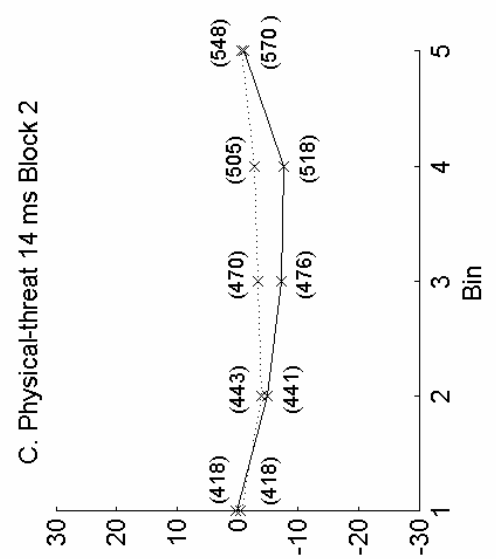
. 2

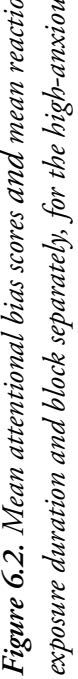




\subsubsection{Response Bias}

\subsection{Mean Response Bias}

Response bias scores were computed by subtracting the mean RT for corresponding emotional-word and response positions from the mean RT for non-corresponding positions. Figure 6.3. shows the resulting scores, pooled across blocks. It suggests that group differences in response bias depended on type of word pair and its exposure duration. An overall ANOVA with type, duration, block, and group as factors revealed that response bias scores of the high-anxious group were more positive than the scores of the low-anxious group (group, $F=5.3$, $p=.024$ ). Furthermore, a trend-level significant interaction suggested that biases depended on the word type (type $\mathrm{x}$ duration $\mathrm{x}$ group, $F(1.99,117.74)=2.3, p=.1$, GreenhouseGeisser corrected $d f s$ ). Therefore, separate ANOVA's were done for each type. None of these revealed any differences in bias between blocks (all $F_{\mathrm{S}}<1.0$ ).

High-anxious $\square$ Low-anxious

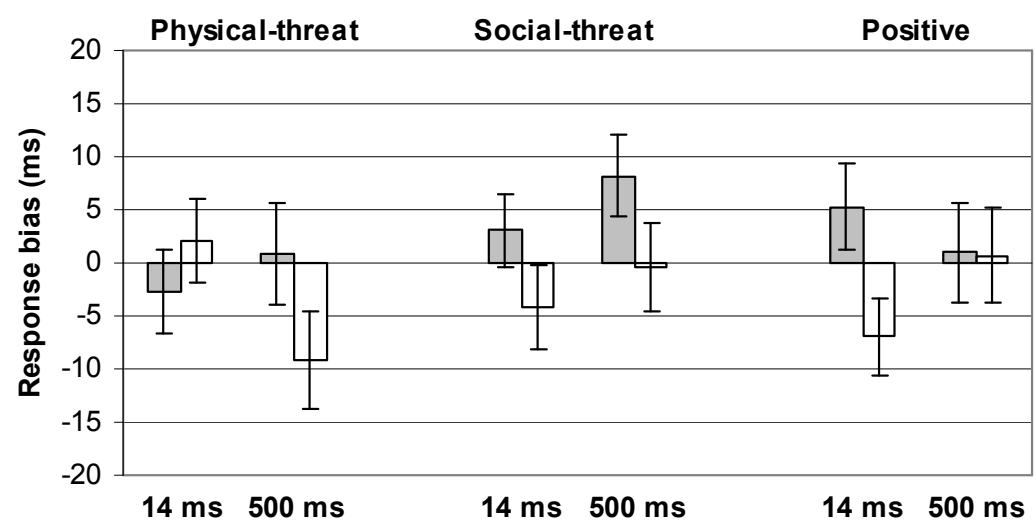

Figure 6.3. Mean response bias scores of the high anxious and the low anxious group for physical-threat words, social-threat words, and positive words for the $14 \mathrm{~ms}$ and the $500 \mathrm{~ms}$ exposure duration, pooled across task blocks.

Physical-threat. Figure 6.3. suggests that for physical-threat words, the most pronounced negative response bias was in the low-anxious group in the $500 \mathrm{~ms}$ condition. Indeed, there was a (marginally significant) duration $\mathrm{x}$ group interaction, $F=2.9, p=.09$. A separate analysis on the $500 \mathrm{~ms}$ condition did not reveal a significant group effect, $F=2.3, p=.14$, but tested against zero the negative bias in 
the low-anxious group was significant, one sample t-tests: $t(30)=-2.0, p=.05$ (high-anxious group, $t(29)=.2, p=.9$ ). In the $14 \mathrm{~ms}$ condition, there was no group effect, $F<1.0$, or bias effect in either group.

Social-threat. For social-threat words, biases were more positive in the highthan in the low-anxious group, $F=4.8, p=.03$, not further modified by duration, $F$ $<1.0$. The high-anxious group showed a positive response bias for social-threat words significantly different from zero, $t(29)=2.0, p=.05$, while there was no bias in the low-anxious group, $t(30)=-.96, \mathrm{p}=.3$.

Positive. For positive words, there were no significant effects of group, $F=2.1, p$ $=.16$, duration, $F<1.0$, or their interaction, $F=2.2, p=.15$.

In sum, consistent with our previous findings (Schrooten \& Smulders, 2007a, b), the high-anxious group showed, in comparison with the low-anxious group, a positive response bias for social-threat words. In addition, the groups differed, albeit weakly, in their response bias scores for physical-threat words presented for $500 \mathrm{~ms}$ : the low-anxious group showed a negative bias, compared to the high-anxious group.

\subsection{Distributional Analyses Response Bias}

For each quantile, response bias scores were computed as described above and polynomial trend analyses were executed in the same way as was done for attentional bias scores.

Physical-threat. The group effect for physical-threat words presented for $500 \mathrm{~ms}$ did not vary across bins (group $\mathrm{x}$ bin-linear / quadratic, $F_{\mathrm{S}}<1.0$; not modified by block). As described above, the mean RT analyses did not reveal any significant effects for physical-threat words presented for $14 \mathrm{~ms}$, and also the distributional analyses did not reveal any effect for these words, $F_{\mathrm{S}}<1.0$.

Social-threat. The group effect for social-threat words (pooled across durations) did not vary across bins (group x bin-linear, $F=1.9, p=.18$; group x bin-quadratic, $F=1.7, p=.2$; not modified by duration and / or block).

Positive. Like the mean RT analyses described above, also the distributional analyses did not reveal significant effects for positive words (group x bin, $F_{\mathrm{S}}<1.0$; not modified by duration and or block).

Taken together, a positive response bias was found for social-threat words in the high-anxious group. In addition, the low-anxious group showed a (weak) negative response bias for physical-threat words presented for $500 \mathrm{~ms}$. These response bias effects did not vary systematically across successive bins. 


\section{Chapter 6}

\subsection{Discussion}

The present probe-classification study provides evidence of content-specific attentional bias and response bias in non-clinical anxiety. (a) The high-anxious, compared to the low-anxious group, showed a positive attentional bias for physicalthreat words presented for $14 \mathrm{~ms}$. The group difference in attentional bias for these words increased with slower RTs. (b) The high-anxious group had also a positive attentional bias for physical-threat words presented for $500 \mathrm{~ms}$ that did not depend on response speed. Furthermore, (c) high-anxious individuals showed a negative attentional bias for social-threat words in the first block, irrespective of exposure duration. (d) For positive words, there was only a weak positive attentional bias in the high-anxious group $(500 \mathrm{~ms} / \mathrm{second}$ block). The attentional bias effects for social-threat and positive words did not vary with response speed. In the present probe-classification task, also response bias effects could be studied, independently of attentional bias. As expected, (e) the high-anxious group showed a positive response bias for social-threat words. (f) The low-anxious group had a weak negative response bias for physical-threat words presented for $500 \mathrm{~ms}$. The response bias effects did not vary with response speed. Below, first the observed attentional bias effects and their time-course will be discussed. Then, the response bias results and their implications will be addressed.

\subsubsection{Anxiety-Related Attentional Bias and Response Speed}

The finding of an anxiety-related positive attentional bias for physical-threat words is consistent with earlier results suggesting an attentional bias towards threat-related information in anxiety (e.g, Fox, 1993a; Mogg et al., 1994; Mogg et al., 1995; Mansell et al., 2002). Our data are also in line with previous findings of attentional bias for suboptimally presented threat-stimuli, taken to suggest a pre-attentive bias for threat-related information in anxiety (e.g., Mogg et al., 1994; Mogg \& Bradley, 1999b). When presented for $14 \mathrm{~ms}$, attentional bias effects for physical-threat words disappeared in the second block. One explanation of this block effect is that awareness may have varied over the course of the task and that the physical-threat (and also social-threat) words presented for $14 \mathrm{~ms}$ became more visible during the second task block. It has been suggested that marginal levels of visibility may result in increased efforts to process briefly presented, masked stimuli, which interfere with automatic, non-conscious influences (Mogg \& Bradley, 1999b, p. 728). This would result in reduced bias effects for stimuli presented close to the awareness threshold, compared to effects for less visible stimuli.

Interestingly, for physical-threat words presented for $14 \mathrm{~ms}$ in the first block, the group difference in attentional bias scores increased as a function of response 
speed. Whereas the attentional bias of the high-anxious group was consistently greater than zero and did not vary with RT, suggesting that the content of these words was already encoded at the fastest responses, the bias of the low-anxious group became more negative with slower responses. When physical-threat words were presented for $500 \mathrm{~ms}$, the group difference in attentional bias was stable across response-bins: the high-anxious group showed an attentional bias towards these words, whereas the low-anxious group showed no bias. Notably, this group effect has the same direction as the effect revealed by slow RTs in the $14 \mathrm{~ms}$ condition, as if it needs 'time to grow'.

Consider the possibility that bias effects are tapped at a later moment in time with slower RTs. Relatively fast responses might then be more sensitive to early bias effects, whereas slower responses might be influenced by later processes. When words were presented for $500 \mathrm{~ms}$, the dot probe was presented later in time than with $14 \mathrm{~ms}$ exposure. Still the direction of the group difference in bias was the same for slow responses in the $14 \mathrm{~ms}$ condition and fast (and slow) responses in the 500 ms condition. The present pattern of results for physical-threat words supports the notion that the main attentional difference between high- and low-anxious individuals lies in how rapidly they direct their attention away or disengage from threat (e.g., Fox, 2004), rather than in early attentional orienting. Our data suggest that, whereas the low-anxious group directed, after some time, their attention away from physical-threat words, the high-anxious group had the tendency to dwell longer on these words. The results suggest that slow RTs in the $14 \mathrm{~ms}$ condition indicate a relative location of attention that, from that point on, does not change over time. The present results for physical-threat words appear to be consistent with the idea that changes of attentional bias effects across different RTs are a reflection of the dynamic time-course of attention.

The finding that attentional bias effects may depend on response speed has some important practical implications. First, distributional RT analyses might inform us about the temporal dynamics of the hypothesized biases, information that is missed if only overall mean RT is analyzed. Furthermore, note that analyses on overall median RTs would reveal similar bias effects as in the third quantile in the present distributional RT analyses; that is an underestimation of the real effect, at least in the present 14-ms exposure condition. Secondly, cut-off points for outlier removal should be chosen cautiously. Whereas it is generally recommended to reduce the potential influence of slow outliers, too strict cut-offs would result in a decrease in sensitivity for relevant effects with slow RTs. Additionally, response deadlines imposed during the task should not be taken too short, for interesting attentional bias effects might be missed with fast RTs.

Besides a positive attentional bias for physical-threat words, the high-anxious group also showed a rather weak negative attentional bias for social-threat words that did not vary with response speed. Unlike previous studies pointing to a positive 


\section{Chapter 6}

attentional bias for stimuli related to social-threat in anxiety (e.g., Bradley et al., 1998; Mansell et al., 2002; Mogg, Philippot, et al., 2004), some recent studies reported also a negative bias for these stimuli. For example, Mogg, Bradley and colleagues (2000) reported a general tendency for undergraduates to show a negative attentional bias for social-threat words relative to neutral words. However, this bias was only significant for those reporting low trait-anxiety yet also high social desirability (repressors). A negative attentional bias has also been observed for emotional faces in patients with social phobia and socially anxious students when under social stress (Chen, Ehlers, Clark, \& Mansell, 2002; Mansell, Clark, Ehlers, \& Chen, 1999; but see Mogg, Philippot, et al., 2004). It may be that our participants, and particularly those with a high-anxious personality trait, felt socially evaluated during the experiment. They were video monitored, and performance feedback was frequently given. However, Mansell et al. (2002) reported that their earlier finding of a negative bias for emotional faces in social anxiety (1999) did not extend to words. This contrasts with the present finding. One reason may be that in Mansell et al.'s (2002) study participants expected to give a video-recorded speech after the task, whereas our participants may have had the impression to be evaluated during the task itself, thus increasing sensitivity to social-threat words. Note that in the present experiment, attentional bias effects for social-threat words were only found in the first block. A simple explanation is that over the course of the experiment, the highanxious group got habituated to the experimental situation and felt less socially stressed.

Most previous dot-probe studies reveal no attentional bias for positive stimuli in high-anxious individuals, suggesting that the reported bias is not due to a general emotionality effect. Also in the present study, we did not observe any bias effect for positive words, except for a very weak positive bias in the high-anxious group when positive words were presented for $500 \mathrm{~ms}$ in the second block. Similarly, Bradley and colleagues (1999) reported a positive attentional bias for happy faces in generalized anxiety disorder, also only in the second half of the task. They speculated that this might, for example, be due to mood regulating or repairing strategies that came into play after some time.

\subsubsection{Anxiety-Related Response Bias}

The main reason for conducting the present study was to examine spatial response bias effects in a probe-classification task. The high-anxious group showed a response bias towards social-threat words, presented for $14 \mathrm{~ms}$ or $500 \mathrm{~ms}$, whereas the lowanxious group showed no bias. These effects did not vary across successive quantiles. No response bias was observed for positive words, indicating that the effects are specific for negative, threat-related information. We propose that the current find- 
ing of an anxiety-related response bias for social-threat words in a probeclassification task adds converging evidence for early preferential processing of these words in anxiety. It is argued that if the social-threat stimulus is preferentially processed, it may act as if presented alone and trigger a Simon effect in responses to probes following this stimulus (e.g. Ivanoff \& Peters, 2000: Simon effects in responses to targets that follow the stimulus conveying the irrelevant spatial information).

Recently, we investigated anxiety-related response bias with a paradigm that differed from the present probe-classification task (Schrooten \& Smulders, 2007a, b). In that paradigm, one emotional and one neutral word were presented and spatial correspondence of emotional word and response systematically varied across trials. The print color of the word pair (red or green) determined response position (top or bottom). Undergraduates exposed to height responded faster when the position of physical-threat words presented for $500 \mathrm{~ms}$ corresponded to the position of the required response than when not, indicating response bias towards physical-threat (Schrooten \& Smulders, 2007a). Furthermore, high-trait-anxious students showed a response bias towards social-threat words when the words were presented for $14 \mathrm{~ms}$ (Schrooten \& Smulders, 2007b). These results are consistent with the present findings, and were also taken as evidence for early preferential processing of threatrelevant information in anxiety. In the paradigm used in these previous studies, emotional as well as neutral words conveyed the relevant information (print color). This information was thus always present at both presumed-attended and unattended locations. We argued that an attentional shift by itself should not lead to faster reactions with one of the response keys (Schrooten \& Smulders, 2007b). In dot-probe tasks, however, the relevant information (conveyed by the probe) is only available at one of the locations, and therefore, probe-classification tasks can be used to study attentional bias, independent of response bias.

In the present study, the high-anxious group showed no significant response bias for physical-threat words, whereas the low-anxious group showed a weak negative bias for these words when presented for $500 \mathrm{~ms}$; the group difference did however not reach significance. This pattern is identical to the negative response bias for physical-threat words shown by the control group in our previous heightexposure study (Schrooten \& Smulders, 2007a). Negative response bias effects are difficult to reconcile with our original account for response bias effects. Negative effects would suggest that the neutral stimulus was preferentially processed at a very early stage. Response bias effects can also be attributed to later biased processing towards or away from the emotional stimulus. For example, behavioral approach / avoidance tendencies might be involved (Schrooten \& Smulders, 2007a). A tendency to avoid emotional stimuli would result in a more negative response bias. The present pattern of results might be explained by suggesting that only the highanxious group preferentially processed physical-threat words (positive response bias), 


\section{Chapter 6}

and that both groups had a tendency to avoid these words (negative response bias). For the high-anxious group, these opposing effects would then cancel each other out. Alternatively, it could be that there is a link between response bias scores and attentional orienting (Schrooten \& Smulders, 2007b). It might be that if attention shifts towards a certain stimulus, motor responses in the direction of this stimulus are also prepared, resulting in a positive response bias (e.g., Nicoletti \& Umiltà, 1989). On the other hand, response bias for social-threat words was opposite to attentional bias, suggesting that they are not simply a reflection of the same single underlying mechanism.

Thus, the current findings demonstrate that threat-related words can yield a response bias in probe-classification tasks. With this result in mind, it may be advisable for future research to apply probe-classification or -detection tasks rather than probe-position tasks in order to investigate pure attentional bias effects. In typical probe-position tasks, we suspect that response bias is confounded with "attentional" bias scores. In a probe-classification task, however, the spatial correspondence of emotional word and response is completely balanced within each level of correspondence between the positions of emotional word and dot, and also in probe-detection tasks there is no systematic spatial correspondence between the emotional stimulus and the response. As a result, in these tasks, we can more confidently attribute the effects of spatial correspondence of emotional word and probe to selective attention, rather than response bias. The opposite attentional bias and response bias effects, as observed for the social-threat words in the present study, will result in reduced 'attentional' bias scores in probe-position tasks compared to classification tasks. Mogg and Bradley (1999a) reported reduced bias scores for threat-related information in anxiety in a probe-position task, compared to a comparable probe-classification task. This reduction could arise from the combination of a purely positive attentional bias and negative response bias.

\subsubsection{Conclusions}

In conclusion, the current data contribute to the research on cognitive mechanisms underlying anxiety in two ways. First, distributional reaction time analyses in an attentional bias task may help to reveal the time-course of attentional bias. Secondly, significant anxiety-related response bias effects were found in a probe-classification task, independent of attentional bias. These response bias effects provide additional evidence for biased processing associated with anxiety. Rather than probe-position tasks, probe-classification or -detection tasks should be used to purely measure attentional bias. 


\section{Chapter 7 \\ Temporal Dynamics of Selective Attention in Non-Clinical Anxiety}

A slightly adapted version of this chapter is submitted as: Schrooten, M. G. S., \& Smulders, F. T. Y. (2007). Temporal dynamics of selective attention in non-clinical anxiety. 


\section{Chapter 7}

\subsection{Abstract}

This experiment examined the temporal characteristics of selective attention in anxiety: its time-course and the influence of processing time. High- and low-traitanxious undergraduates performed a task similar to the dot-probe task. Emotionalneutral word pairs were presented for 14 or $500 \mathrm{~ms}$; the probe appeared at word onset or after $514 \mathrm{~ms}$. Exposure duration (processing time) and word-probe onsetasynchrony (SOA; moment at which bias was tapped) were manipulated independently. Faster responses when emotional stimulus and probe spatially corresponded than when not (positive bias) were taken to reflect attentional bias for the emotional stimulus. High-anxious participants showed a more positive bias for physical-threat, social-threat (first half of the task), and positive words (second half). Bias effects elicited by these word types differed in temporal characteristics, suggesting specificity of anxiety-related bias. Differences between bias scores to threat words presented for 14 and $500 \mathrm{~ms}$, given $0-\mathrm{ms}$ SOA, suggest that the probe does not uniquely tap the momentary allocation of attention at probe onset.

\subsection{Introduction}

In the last two decades, researchers have been challenged to specify the precise nature of selective attention in anxiety and to develop appropriate methodologies and measures to study its characteristics (for an up-to-date review, see Yiend, 2004a). This work has been largely motivated by the suggestion that information processing biases, and especially attentional biases, underlie several problems associated with (clinical) anxiety (for an overview of cognitive theories on anxiety, see Mogg \& Bradley, 1998; Mathews \& Mackintosh, 1998). Therefore, detailed knowledge of the cognitive mechanisms in anxiety may help to improve clinical treatment. The focus of the present paper is on the temporal aspects of attentional bias in non-clinical anxiety.

Modifications of Posner's (1980) spatial cueing paradigm have been widely used to study anxiety-related attentional bias, its specificity, time-course and automaticity (Mogg \& Bradley, 1998; Fox, 2004). During dot-probe tasks, for instance, a stimulus pair, containing one emotional and one neutral word or picture, is presented and followed by a probe at a location previously occupied by one of the members of the pair. Cueing studies have consistently shown that responses are faster to probes at attended rather than unattended locations (e.g., Posner, Snyder, \& Davidson, 1980). Building on this finding, in dot-probe studies, faster responses when emotional stimulus and probe appear in the same position than when they occur in different positions have been taken to reflect attentional bias for the emotional 
stimulus; that is selective attention to the emotional stimulus, in preference to a simultaneously presented neutral one.

In dot-probe research, it has been common to present stimulus pairs for 500 ms. In general, dot-probe studies employing this exposure duration have revealed an attentional bias for threat-relevant stimuli, in anxiety patients (e.g., MacLeod, Mathews \& Tata, 1986; Mogg, Philippot, \& Bradley, 2004) and in people with a high-anxious personality trait (e.g., Broadbent \& Broadbent, 1988; Fox, 1993a; Mogg \& Bradley, 1999a) especially when under stress (e.g., MacLeod \& Mathews, 1988; Mogg, Mathews, Bird \& MacGregor-Morris, 1990; Mogg, Bradley, \& Hallowell, 1994). A bias has also been reported for low-anxious people, but only for strongly threatening pictures (Mogg, McNamara et al., 2000; Wilson \& MacLeod, 2003). They had either no bias or even a negative bias for less threatening stimuli (e.g., MacLeod \& Mathews, 1988; Fox, 1993a; Mogg et al., 1994).

An exposure duration of $500 \mathrm{~ms}$ is relatively long and readily allows awareness of stimulus content. Therefore, this presentation time may enable the operation of strategic processes, occurring after information entered awareness. To investigate whether anxiety-related attentional bias occurs independent of awareness, as predicted by Williams Watts, MacLeod, and Mathews (1988), dot-probe studies were carried out in which stimulus pairs were presented very briefly (e.g., $14 \mathrm{~ms}$ ), followed by a mask. Even under these conditions of restricted awareness, significant attentional bias effects have been observed for threat, both in sub-clinical and pathological anxiety (e.g., Mogg et al., 1994; Mogg, Bradley \& Williams, 1995; Bradley, Mogg, \& Lee, 1997; Mogg \& Bradley, 1999b, 2002; Fox, 2002). This has been taken as evidence for an automatic preconscious processing bias in anxiety.

Inspired by evidence that the attention system can be broken down in separate components (engagement, shifting and disengagement; Posner \& Petersen, 1990), the question has been raised as to how these subsystems are biased in anxiety (e.g., Mogg, Bradley, de Bono \& Painter, 1997). The aforementioned dot-probe results for stimuli presented for 14 or $500 \mathrm{~ms}$ have been taken as evidence for an anxietyrelated bias in initial orienting and early attentional engagement (e.g., Mogg et al., 1997; Bradley, Mogg, Falla \& Hamilton, 1998)..$^{37}$ To investigate whether anxious individuals, after initial orienting, direct their attention away (avoidance) or maintain their attention on threat (difficult disengagement), dot-probe studies have been conducted in which stimulus duration has been manipulated systematically (Mogg,

\footnotetext{
${ }^{37}$ Fox (e.g., 2004) pointed out that when stimulus pairs are presented for $500 \mathrm{~ms}$, it is actually possible that participants switch covert attention several times between the two stimuli and that, therefore, it may be that bias effects observed with this presentation time do not reflect an initial shift in attention. Researchers assuming that bias effects assessed with 500-ms exposure reflect initial orienting (Bradley et al., 1998; Mogg, Philippot, et al., 2004; Mogg, Bradley, et al., 2004; Lees et al., 2005) refer to Bradley, Mogg and Millar (2000)'s finding of concordance between the bias derived from dot-probe data (with 500-ms exposure) and the direction of initial shift in overt gaze to emotional faces.
} 
Bradley, Miles, \& Dixon, 2004; Koster, Verschuere, Crombez, \& Van Damme, 2005; Mogg et al., 1997; Bradley et al., 1998; Bradley et al., 1997; Mogg, Philippot, et al., 2004; Lees, Mogg, \& Bradley, 2005; Mogg \& Bradley, 2006). The course and time scale of attentional bias effects seem to depend on the threat value of the stimuli and the anxiety group under consideration (clinical vs. non-clinical; state vs. trait; general anxiety vs. specific fear).

In many dot-probe studies that manipulated stimulus duration, either to investigate the role of awareness or to map the time-course of attentional bias, probes appeared immediately after the stimulus pair or mask. Thus, longer exposure was paired with a larger interval between the stimulus pair and the probe (stimulus onset asynchrony; SOA). If it is assumed that bias effects are 'tapped' at probe onset, then the larger the SOA, the later the bias is tapped in its time-course. Recent research into selective attention highlights the importance of considering both SOA and exposure duration as separate temporal factors. In tasks different from the dot-probe task, effects of attentional orienting have been found to depend strongly on the combination of cue duration and cue-target SOA (Collie, Maruff, Yucel, Danckert, \& Currie, 2000; Gibson \& Bryant, 2005; McAuliffe \& Pratt, 2005). A confounding of exposure duration and SOA in the dot-probe task renders it impossible to isolate the effects of processing time and moment of tapping.

To our knowledge, there are three published dot-probe studies that examine the influence of processing time on anxiety-related bias by manipulating exposure duration, while keeping SOA constant. First, Mathews, Ridgeway, and Williamson (1996) presented words either for $500 \mathrm{~ms}$ or for three times $50 \mathrm{~ms}$, alternated with 50-ms masks (restricted awareness). They observed for panic patients a bias for physical-threat words in both cases. Note that their restricted awareness condition deviates from the typical backward masking procedure. Also, in contrast to most other dot-probe studies, participants also had to read aloud a digit appearing between the words. Second, Egloff and Hock (2003) presented words for either 500 $\mathrm{ms}$ or for $50 \mathrm{~ms}$ followed by a 450-ms mask. Also in this study, participants had to read a central digit. With 500-ms exposure, high-trait-anxious participants showed the expected bias for social-threat words, whereas low-anxious participants directed their attention away. However, with 50 -ms exposure, there were no significant differences in bias between these groups. Finally, Luecken, Tartaro, and Appelhans (2004) displayed words for either about $500 \mathrm{~ms}$ or for $20 \mathrm{~ms}$ followed by a 500 -ms mask. These exposure conditions were, in contrast to what is common in dot-probe research, not intermixed, but presented in separate blocks. For participants completing the long exposure block first, with short exposure, there was evidence for a positive correlation between trait-anxiety and bias for social-threat words. With long exposure, this correlation was negative.

To date, there is, to our knowledge, no dot-probe study on anxiety in which SOA has been varied, while keeping stimulus duration constant. Yet, this procedure 
has been used successfully to map the time-course of comparable phenomena like covert attentional orienting (e.g, Posner \& Cohen, 1984), priming (e.g., Hermans, De Houwer \& Eelen, 2001; Richards \& French, 1992) and stimulus-response compatibility effects (e.g., Simon, Acosta, Mewaldt \& Speidel, 1976). Effects at short SOAs (e.g., shorter than $200 \mathrm{~ms}$ ) are thought to reflect the influence of early, fast-acting cognitive processes. Effects at longer SOAs are thought to reflect the influence of strategic processes, building up as time progresses across a trial. By systematically varying the SOA, it should be possible to specify the points in time at which a particular effect starts to occur, to dissipate or to reverse.

The present experiment was designed to further investigate the temporal dynamics of attentional bias in non-clinical anxiety. Specifically, the time-course of anxiety-related attentional bias effects and the influence of processing time on these effects were examined. Undergraduates reporting high or low levels of trait-anxiety performed a task highly similar to the dot-probe task. Emotional-neutral word pairs (physical-threat, social-threat, positive) were presented for either 14 or $500 \mathrm{~ms}$. Task-relevant information (the probe) appeared either immediately at word onset or after 514 ms. Hence, the present study extends previous dot-probe studies in two respects. First, attentional bias was not only tapped later in its time-course, when the words had already disappeared (514-ms SOA), but also at word onset (0-ms SOA). Second, exposure duration and SOA were de-confounded and the effect of either factor could be assessed independently. Sub-clinically anxious individuals may be characterized by controlling strategies counteracting initial automatic bias effects (e.g., Fox, 1996, 2002). Alternatively, it may be that high-anxious individuals, even with 500 -ms processing time, maintain their attention on the threat-relevant stimulus, whereas low-anxious individuals disengage their attention from it (Cf. Fox, Russo, Bowles, \& Dutton, 2001, Exp. 2).

Some of our conditions strongly resemble conditions used by others. These are conditions during which words are presented for $500 \mathrm{~ms}$ in combination with an SOA of 514 ms (e.g., Mogg \& Bradley, 1999a) or in which stimuli were presented under conditions of restricted awareness followed by a probe after about $500 \mathrm{~ms}$ (Mathews et al., 1996; Egloff \& Hock, 2003; Luecken et al., 2004). In these cases, we expected to replicate the prior finding. With 500-ms exposure and 514-ms SOA, the high-anxious group should show a content-specific attentional bias for threatrelevant words, relative to the low-anxious group. With 14-ms exposure and 514-ms SOA, we had no clear hypothesis. The few previous studies with brief, masked exposure and 500-ms SOA differed considerably in methodology and yielded mixed results. If anything, we expected an anxiety-related bias for threat-relevant words, consistent with Mathews et al. (1996) and Luecken et al. (2004). 


\subsection{Method}

\subsubsection{Participants}

We selected undergraduates reporting extremely high or low levels of trait-anxiety, and low levels of defensiveness. At an initial screening session, trait-anxiety and defensiveness were assessed with the Spielberger State-trait Anxiety Inventory (STAI-trait; Van der Ploeg, Defares \& Spielberger, 1980) and the Marlowe-Crowne Social Desirability scale (SDS; Hermans, 1967), respectively. Those scoring 40 or more, or 32 or less on the STAI-trait, and scoring 18 or less on the SDS were invited to take part. Altogether, 24 high-anxious and 26 low-anxious undergraduates were tested. ${ }^{38}$ All were native Dutch speakers, non-dyslexic, and reported (corrected to) normal vision and color vision. They participated on an informed consent basis and received $€ 12.5$. The study was approved by the department's Ethics Committee.

\subsubsection{Materials}

The critical stimuli consisted of 32 social-threat (e.g., stupid, criticism), 32 physicalthreat (e.g., tumor, lethal), and 32 positive (e.g., paradise, cheerful) Dutch words, individually matched with neutral words in terms of both length and frequency (CELEX; Baaijen, Piepenbrock, \& Gulikers, 1995). Ninety-six neutral-neutral pairs were created to act as fillers. The critical stimuli were mainly drawn from published lists (e.g., MacLeod et al., 1986). Word length ranged from three to ten letters.

The affective content of all words was rated by eight staff members ( 3 men; all Dutch) on a visual analogue scale. Physical-threat words, social-threat words, and neutral words paired with them were rated on their association with physical-threat and social-threat, respectively (from 'not at all' to 'extremely'). The valence of positive words, neutral words paired with them, and filler words was rated on a bipolar scale (from 'very negative' to 'very positive'). The mean rating difference ( $z$ scores) between the members of any physical-threat-neutral pair was at least 1.3 $($ mean $=1.9)$, of any social-threat-neutral pair at least $1.4($ mean $=1.8)$, of any positive-neutral pair at least $1.4($ mean $=1.8)$ and of any neutral-neutral pair between -.5 and $.5($ mean $=.02)$.

\footnotetext{
${ }^{38}$ To verify that participants were consistent, the STAI was again administered at the experimental session. Eighteen additional participants were excluded because they scored high on STAI-trait during pre-screening, but below 40 during the experiment, or low during pre-screening, but above 32 during the experiment.
} 
During practice trials, additional word pairs were presented. Mirrored, upsidedown upper-case consonants, numbers and symbols were used as backward mask. Masks were unique to each word, length- and color-matched with the preceding word, and presented for $14 \mathrm{~ms}$.

\subsubsection{Apparatus}

Task presentation and response registration were controlled by a Dell Optiplex GX260 computer running ERTSVIPL (Berisoft Cooperation, Frankfurt, Germany).

\subsubsection{Procedure}

Participants were tested individually, seated at a viewing distance of about $60 \mathrm{~cm}$ from the monitor in a dimly lit room. They were video-monitored and could communicate through a two-way communication system. They were informed that the study investigated the relationship between concentration and performance. The real purpose of the experiment was explained after all participants had been tested.

Each trial started with the 500-ms presentation of a white central fixation cross on a dark background. Then, a backwardly masked word pair in upper case letters (non-proportional font, $0.5 \mathrm{~cm}$ high) appeared, with one word above, the other below the center of the screen $(3 \mathrm{~cm}$ between top upper word and bottom lower word). In half of the trials, the emotional word was presented at the upper position, in the other half at the bottom position. In half of the trials, words appeared for 14 $\mathrm{ms}$, in the other half for $500 \mathrm{~ms}^{39}$ After the mask, a dot $(3 \times 2 \mathrm{~mm})$ appeared at each location previously occupied by a word. Color information (red or green) was added to the display to determine the response. In half of the trials, word-color SOA was $0 \mathrm{~ms}$ (one of the words colored), in the other half it was $514 \mathrm{~ms}$ (words in white, one of the dots colored). The color appeared equally likely at the upper or lower position, and thus took the role of the dot-probe in conventional dot-probe tasks. Figure 7.1 depicts the resulting four combinations of SOA and exposure duration.

All participants had to respond as quickly and accurately as possible by pressing the top key with the right index finger to red and the bottom key with the left index

\footnotetext{
${ }^{39}$ Similar exposure durations and masks were used as in most previous dot-probe experiments on the role of awareness (e.g., Mogg et al, 1994, 1995). In those previous tasks, (objective) awareness checks were presented, suggesting that participants' awareness of the presence or lexical content of the masked words was very restricted.
} 


\section{Chapter 7}

finger to green. ${ }^{40}$ No instruction was given to process the words in any particular way. Participants were encouraged to fixate the cross from its presentation until the response. The dots were displayed until a response was made or for a max. of 1200 ms. The inter-trial interval varied randomly between 500 and $1250 \mathrm{~ms}$. In case of an error, a beep and an error message (showing the instruction) lasting $250 \mathrm{~ms}(+$ $2500 \mathrm{~ms}$ pause) were inserted. Visual feedback was given if the participant responded too slowly (reaction time $(\mathrm{RT})>800 \mathrm{~ms})$ or prematurely $(\mathrm{RT}<150 \mathrm{~ms})$.

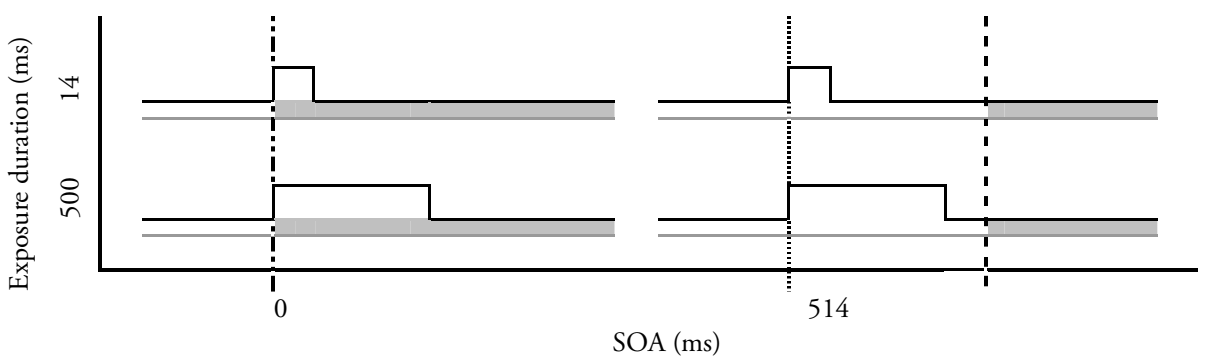

Figure 7.1. The four combinations of word-color onset asynchrony (SOA; X-axis) and exposure duration of the words (Y-axis). Grey shading depicts relevant color (red/green).

All participants completed two task blocks, each consisting of 36 practice trials, followed by 768 experimental trials. Per block, the design was completely balanced and each word pair was presented once for each exposure duration/SOA combination. The experimental trials were presented in a different random order for each participant. Performance feedback (mean RT) was given every 32 trials during breaks terminated by the participants.

After the computerized task, the participants completed Dutch versions the STAI-trait, STAI-state, Beck Depression Inventory (BDI; Beck, Ward, Mendelson, Mock, \& Erbaugh, 1961) and SDS.

\footnotetext{
${ }^{40}$ Correspondence of emotional word position and response position was completely balanced within each level of correspondence between emotional word position and color position (i.e., attentional bias). So, attentional bias effects were not confounded with any effects of correspondence between emotional word and response.
} 


\subsection{Results}

\subsubsection{Group Characteristics}

Three participants were excluded because of a high percentage ( $>9 \%=$ mean +2 $\mathrm{SD})$ of errors or slow responses $(>800 \mathrm{~ms})$. The final groups differed in traitanxiety, state-anxiety, depression, and age, but not in social desirability or gender ratio (see Table 7.1.; men:women ratios for the low- and the high-anxiety groups were 5:20 and 1:21, respectively, $\left.\chi^{2}(47,1)=2.5, n s.\right)$.

Table 7.1. Group sizes, mean age, and mean scores (SD in brackets) on the STAI Trait and State Anxiety scales, the Beck Depression Inventory (BDI), and Marlowe-Crowne Social Desirability Scale (SDS).

\begin{tabular}{|c|c|c|c|c|}
\hline \multirow{3}{*}{ Measure } & \multicolumn{2}{|c|}{ Group } & \multirow{3}{*}{$t$} & \multirow{3}{*}{$d f$} \\
\hline & High-anxious & Low-anxious & & \\
\hline & $n=22$ & $n=25$ & & \\
\hline Age & $\begin{array}{l}20.9(1.4) \\
\text { (range 20-26) }\end{array}$ & $\begin{array}{l}19.8(1.7) \\
\text { (range 19-27) }\end{array}$ & $2.4^{*}$ & 45 \\
\hline Trait-anxiety & $45.5(6.3)$ & $27.2(2.5)$ & $12.8^{* *}$ & $26.7^{\mathrm{a}}$ \\
\hline State-anxiety & $40.0(7.9)$ & $26.8(4.1)$ & $7.0^{* *}$ & $30.8^{\mathrm{a}}$ \\
\hline BDI & $11.5(5.4)$ & $1.4(1.7)$ & $8.4^{* *}$ & $24.6^{\mathrm{a}}$ \\
\hline SDS & $13.9(4.6)$ & $14.9(3.1)$ & -.9 & 45 \\
\hline
\end{tabular}

Note. STAI - Spielberger State-Trait Anxiety Inventory.

a Equality of variances in the anxiety groups could not be assumed (Levene's test)

${ }^{*} p<.05{ }^{* *} p<.001$

\subsubsection{Behavioral Results}

Incorrect responses ( $4.7 \%$ of all trials) and correct responses with latencies longer than 800 or shorter than $150 \mathrm{~ms}(.6 \%$ of correct trials) were eliminated. In the final sample $(N=47)$, on average, $95.0 \%$ (range: $92-98 \%$ ) of the data remained for analyses. There were no significant differences in error or outlier rates between blocks or anxiety groups.

Analyses were performed on median RTs ${ }^{41}$ All reported $p$-values are two-tailed. For ANOVA results involving word type (3), $F$ - and $p$-values reflect the Green-

\footnotetext{
${ }^{41}$ The pattern of results obtained when mean RTs were analyzed was essentially the same as for the median RT results.
} 
house-Geisser correction for non-sphericity, and uncorrected $d f s$ are $(2,44)$. For other ANOVA results, $d f$ are $(1,45)$.

First, median RTs were subjected to an ANOVA with block (first, second), SOA $(0,514 \mathrm{~ms})$, duration $(14,500 \mathrm{~ms}$ exposure), type (physical-threat, socialthreat, positive words), emotional word position (top, bottom), color position (top, bottom), response position (top, bottom) as within-subject factors and anxiety (high-, low-anxious) as between-subject factor. In general, responses were somewhat faster during the second block $(397 \mathrm{~ms})$ than during the first block $(420 \mathrm{~ms}), F=$ $115.2, p<.00001$, and when pressing the top key (398 ms) than when pressing the bottom key (419 ms), $F=110.0, p<.00001$. Furthermore, responses were fastest in the 14/514 duration / SOA condition (398 ms), slowest in the 14/0 condition (415 $\mathrm{ms}$ ), and in between for the 500/514 (411 ms) or 500/0 conditions (410 ms) [SOA: $F=10.5, p=.002$; duration: $F=9.3, p=.004$; SOA x duration: $F=46.2, p<$ $.0001]$. These small differences between duration/SOA conditions are likely caused by the combination of a general alerting effect in 514-ms SOA conditions (the word pair acting as a warning for later color onset) and some interference if color onset occurs near the offset of the word pair.

More relevant are the results involving an interaction between emotional word position and color position, reflecting attentional bias. To simplify the interactions, attentional bias scores were computed by subtracting the mean RT when the probe color appeared on the same position as the emotional word (corresponding) from the mean RT when the color and the emotional word appeared at different locations (non-corresponding). Positive values indicate faster responses on corresponding than on non-corresponding trials and are taken to reflect attentional bias for the (location of the) emotional stimulus. Figure 7.2 (a-e) displays the mean attentional bias scores.

Attentional bias scores were analyzed using an ANOVA with block, SOA, duration, type (3) and anxiety as factors. There was a block $\mathrm{x}$ type $\mathrm{x}$ anxiety interaction, $F=3.4, p=.04$, superseding a main effect of anxiety, $F=6.7, p=.01$, and a block x type interaction, $F=5.01, p=.01$. This three-way interaction indicates that the high-anxious group, compared to the low-anxious group, had a more positive attentional bias score for physical-threat words, for social-threat words during the first task block and for positive words during the second block (see Figure 7.2 a, b). ${ }^{42}$

\footnotetext{
${ }^{42}$ In addition, there was a significant duration $\mathrm{x}$ type interaction, $F=3.7, p=.03$, suggesting that attentional bias scores for physical-threat and especially social-threat words tended to be more positive with 14-ms than with 500-ms exposure (physical-threat: -.9 ms vs. $2.3 \mathrm{~ms}$; social-threat: $-4.1 \mathrm{~ms}$ vs. $4.3 \mathrm{~ms}$ ), whereas bias scores for positive words showed the opposite pattern $(2.7 \mathrm{~ms}$ vs. $-.35 \mathrm{~ms})$.
} 


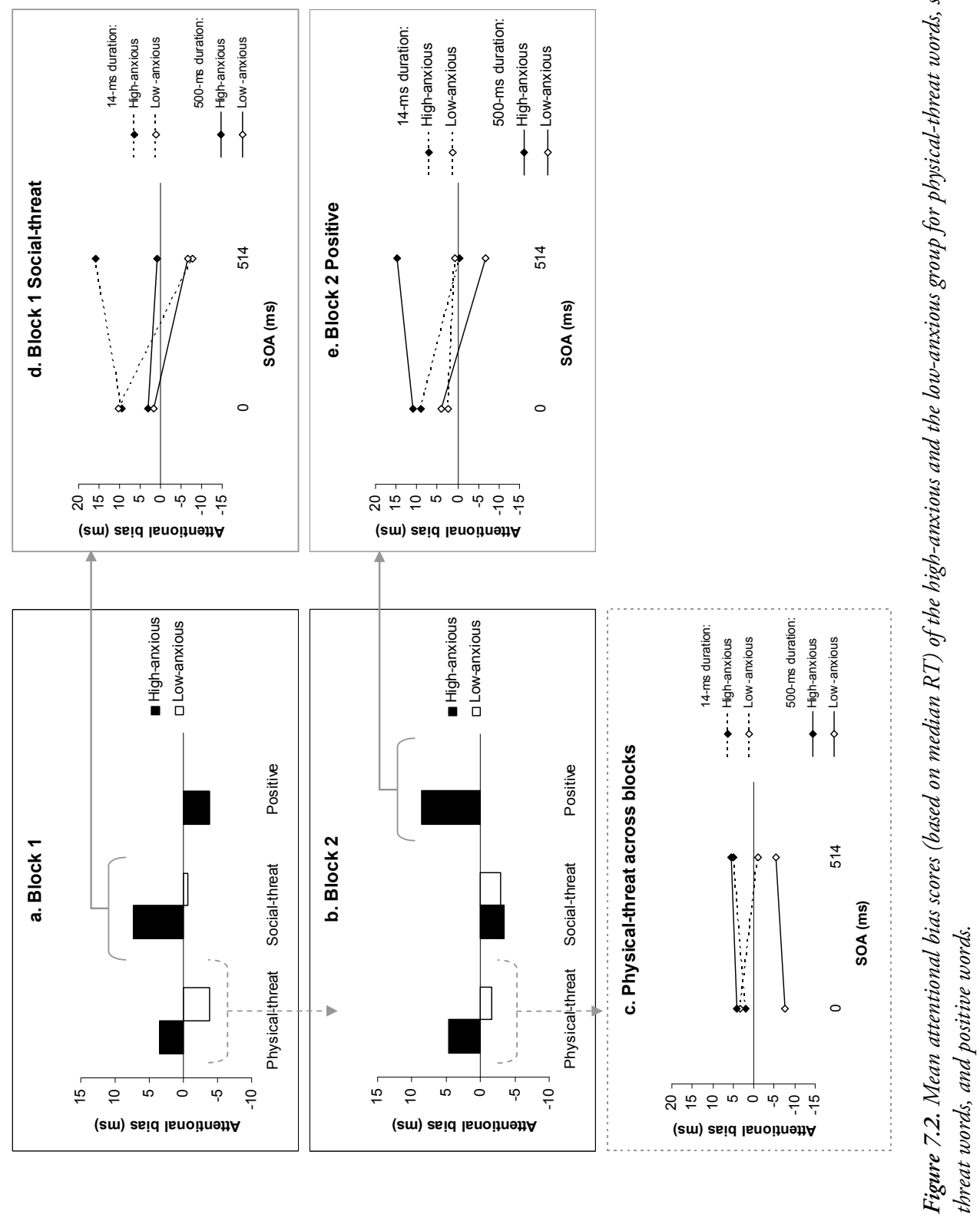




\section{Chapter 7}

To assess the specific temporal dynamics of the bias elicited by the different word types, attentional bias scores were, for each word type separately, entered into an ANOVA with block, SOA, duration and anxiety as factors. Table 7.2. presents the statistics.

Table 7.2. Results of ANOVAs on attentional bias scores (based on median RTs) with block (first, second), SOA (0, $514 \mathrm{~ms})$, exposure duration (14, $500 \mathrm{~ms}$ ) and anxiety (high-, low-anxious) as factors, for each word type separately.

\begin{tabular}{|c|c|c|c|c|c|c|}
\hline & \multicolumn{2}{|c|}{ Physical-threat } & \multicolumn{2}{|c|}{ Social-threat } & \multicolumn{2}{|c|}{ Positive } \\
\hline & $F$ & $p$ & $F$ & $p$ & $F$ & $p$ \\
\hline anxiety & 4.04 & $.05^{*}$ & 1.79 & .19 & .59 & .45 \\
\hline x SOA & .55 & .46 & .71 & .40 & .02 & .89 \\
\hline $\mathrm{x}$ duration & 1.98 & .17 & .41 & .52 & .99 & .33 \\
\hline $\mathrm{x}$ SOA $\mathrm{x}$ duration & .62 & .44 & .27 & .61 & 1.34 & .25 \\
\hline block $\mathrm{x}$ anxiety & .04 & .84 & 1.81 & .19 & 5.72 & $.02^{*}$ \\
\hline $\mathrm{xSOA}$ & .15 & .70 & 2.94 & $.09^{*}$ & .53 & .47 \\
\hline $\mathrm{x}$ duration & .01 & .94 & .10 & .75 & .63 & .43 \\
\hline $\mathrm{x}$ SOA $\mathrm{x}$ duration & .07 & .80 & .79 & .38 & .46 & .50 \\
\hline grand mean & .17 & .68 & .01 & .94 & .63 & .43 \\
\hline SOA & .05 & .83 & .84 & .36 & 1.90 & .18 \\
\hline duration & 1.01 & .32 & 5.28 & $.03^{*}$ & 1.01 & .32 \\
\hline SOA $x$ duration & .23 & .63 & .35 & .56 & .02 & .90 \\
\hline block & .31 & .58 & 4.18 & $.05^{*}$ & 5.92 & $.02^{*}$ \\
\hline x SOA & .08 & .77 & .89 & .35 & .05 & .82 \\
\hline $\mathrm{x}$ duration & .01 & .93 & .13 & .72 & .01 & .94 \\
\hline x SOA x duration & 3.28 & $.08^{\#}$ & .25 & .62 & .19 & .66 \\
\hline
\end{tabular}

Note. $d f \mathrm{~s}$ are $(1,45)$.

${ }^{* *} p \leq .01 \quad * p \leq .05 \quad * p \leq .10$

Physical-threat words. The high-anxious group showed a more positive attentional bias than the low-anxious group. This effect was not modified by SOA or duration. There were no significant relevant block effects. However, visual inspection of the bias scores (Figure 7.2c) strongly suggests that the group difference for physical-threat words occurred at both moments of tapping (i.e., SOA), but only when words were presented for $500 \mathrm{~ms}$. For each exposure duration separately, bias scores were subjected to an ANOVA with SOA and anxiety as factors. In the 500 ms condition, there was a significant group difference, $F=5.1, p=.03$, and no 
effect of SOA (SOA x anxiety, SOA: $F_{S}<1.0$ ). In the 14-ms condition, there were no effects at all, $F_{S}<1.5, p s>.24 .{ }^{43}$

In sum, in line with previous dot-probe studies, an anxiety-related bias was observed for physical-threat words presented for $500 \mathrm{~ms}$ with 514-ms SOA, with the high-anxious group having a more positive bias than the low-anxious group. In addition, a similar group difference was observed with 500-ms exposure, but 0 -ms SOA. Finally, the bias effects tended to be larger with $500-\mathrm{ms}$ than with $14-\mathrm{ms}$ exposure.

Social-threat words. The main effect of duration suggests that attentional bias scores with 14-ms exposure were in general more positive than those in the 500-ms condition. More relevant is the trend-level significant block x anxiety x SOA interaction, superseding a significant block effect. For each block separately, attentional bias scores were entered into an ANOVA with anxiety and SOA as factors. Table 7.3. presents the statistics.

During the first block, the high-anxious group showed a more positive attentional bias than the low-anxious group. Interestingly, this anxiety effect was superseded by a significant anxiety x SOA interaction. For both SOAs, the effects were larger in the 14-ms than in the 500-ms condition. ${ }^{44}$ As can also be seen in Figure 7.2d, with 0 -ms SOA, both groups had a positive bias for social-threat words. With 514-ms SOA, the high-anxious group still showed a positive bias, whereas the bias in the low-anxious group had reversed. ${ }^{45}$ During the second block, there were no significant anxiety or SOA effects.

In sum, there was an anxiety-related bias for social-threat words in the first task block. The group difference for this bias increased when the bias was tapped later in time. When the bias was tapped at word onset ( $\mathrm{SOA}=0 \mathrm{~ms}$ ), both groups tended to

\footnotetext{
${ }^{43}$ Bias scores per group were also tested against zero (no bias). In the 500-ms condition, the low-anxious group showed a negative bias for physical-threat words, significantly different from 0 (across SOAs: $t(24)=-2.1, p=$ .04; 0-ms SOA: $t(24)=-1.9, p=.07$; 514-ms: $t(24)=-1.6, p=.12)$. The high-anxious group showed no significant bias for these words, $.3<t(21) s<1.3$, $p s>.2$. In the 14-ms condition neither group showed a bias significantly deviant from $0,-.25<t s<1.1, p s \geq .3$.

${ }^{44}$ 14-ms duration: 0-ms SOA: grand mean: $F=5.1, p=.03$, anxiety: $F<1.0 ; 514$-ms: grand mean: $F=1.7$, $p=.3$, anxiety: $F=8.7, p=.005$; 500-ms duration: 0 -ms and 514-ms SOA, both grand mean and anxiety: $F<$ 1.0 .

${ }^{45}$ With 0 -ms SOA, the high-anxious nor the low-anxious group showed a bias significantly different from 0 , $t(21)=1.4, p=.18$ and $t(24)=1.4, p=.17$, respectively. However, with 514-ms SOA, the high-anxious group showed a significant positive bias, $t(21)=2.1, p=.045$, whereas the low-anxious group showed a trend-level significant negative bias, $t(24)=-8.6, p=.075$. Bias scores tested vs. 0: 14-ms duration: 0-ms SOA: lowanxious: $t(24)=1.6, p=.12$; high-anxious: $t(21)=1.6, p=.12$; 514-ms SOA: low-anxious: $t(24)=1.5, p=$ .14 ; high-anxious: $t(21)=2.5, p=.02 ; 500-\mathrm{ms}$ duration: neither group had a bias significantly deviating from $0:-.9<t s<.4, p s>.35$.
} 


\section{Chapter 7}

have a positive bias for social-threat words. Whereas the high-anxious group continued to show a positive bias for these words after $514 \mathrm{~ms}$, the low-anxious group had a negative bias. These effects were larger with 14-ms than with 500-ms exposure.

Table 7.3. Results of ANOVA on attentional bias scores (based on median RTs) for social-threat words with SOA (0, 514 ms) and anxiety (high-, low-anxious) as factors, for each block separately. Significant relevant interactions were broken down per SOA or per anxiety group and statistics for follow-up ANOVAs are given in italics.

Social-threat

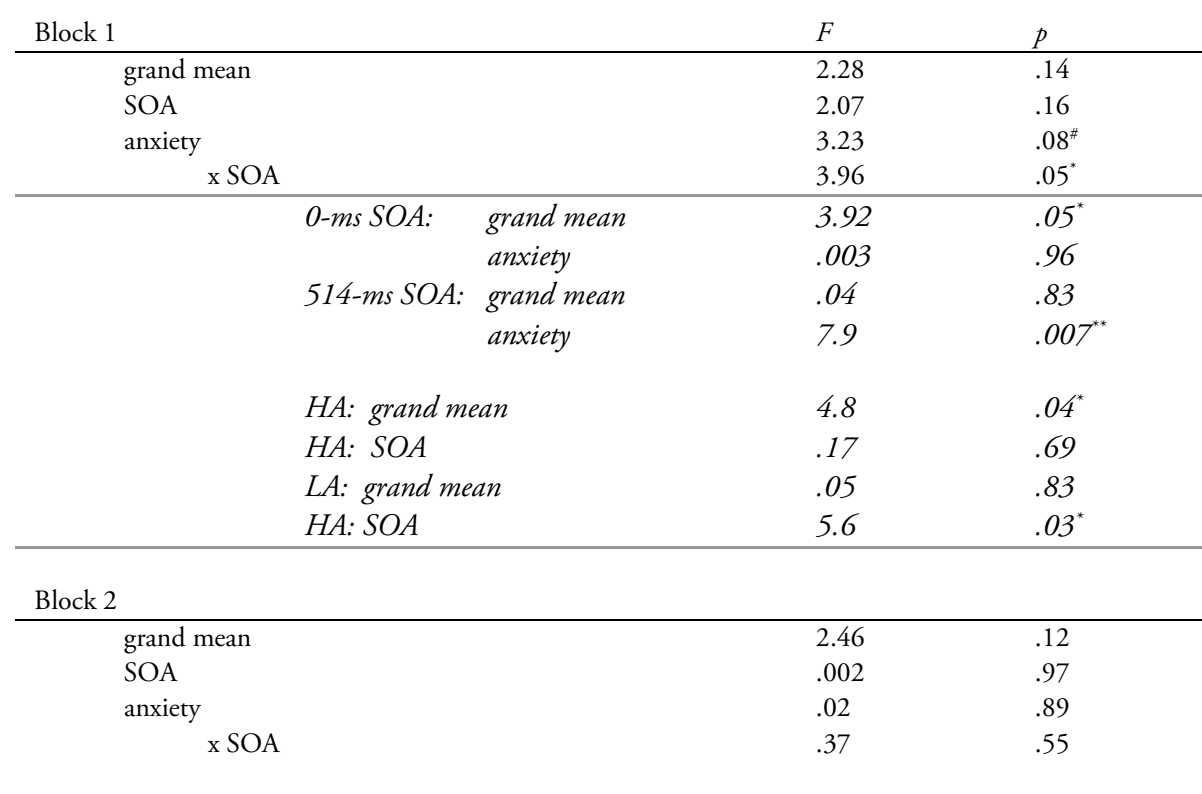

Note. $\mathrm{HA}=$ High-anxious; LA = Low-anxious; $d f$ s are $(1,45)$, except for High-anxious $(1,21)$, Low-anxious $(1,24)$.

${ }^{* *} p \leq .01 \quad * p \leq .05 \quad * p \leq .10$

Positive words. There was a significant block $\mathrm{x}$ anxiety effect, not modified by SOA or duration. For each block separately, attentional bias scores were entered into an ANOVA with anxiety as factor. During the first block, there were no significant effects, $F_{\mathrm{S}}<1.2$, $p \mathrm{~s}>2.8$. During the second block, the high-anxious group showed a more positive attentional bias than the low-anxious group, $F=4.0, p<$ .05 . Visual inspection of Figure 7.2e suggests that this effect only occurred when words were displayed for $500 \mathrm{~ms}$, and more so when color onset delay was $514 \mathrm{~ms}$. A separate ANOVA for the second block revealed a trend-level significant anxiety $\mathrm{x}$ 
SOA x duration effect, $F=2.6, p=.1$, mildly supporting this observation. Further ANOVAs per exposure duration showed that in the 500-ms condition, there was a trend-level significant group difference, $F=3.7, p=.06$, not modified by SOA, $F=$ $2.05, p=.16$ (SOA: $F<1.0$ ), whereas in the 14-ms condition, there were no significant effects at all, $F_{\mathrm{S}}<1.4, p s>.25$. Independent t-tests revealed only a significant group difference in the 500/514 condition, $t(45)=2.1, p=.04(500 / 0: t(45)=.9, p$ $=.4 ; 14 / 0: t(45)=.95, p=.35 ; 14 / 514: t(45)=.15, p=.9) \cdot{ }^{46}$

In sum, during the second task block, the high-anxious group, compared to the low-anxious group, showed a positive bias for positive words presented for $500 \mathrm{~ms}$. As for physical-threat words, the group difference occurred both with 514-ms and with 0 -ms SOA; the difference was slightly larger with 514-ms SOA. Finally, with 14-ms exposure, there were no significant effects.

\subsection{Discussion}

The present experiment focused on the temporal characteristics of attentional bias in non-clinical anxiety. A paradigm similar to the dot-probe task was used, during which word pairs and a probe (color) were presented. Importantly, in the two conditions that resemble previous studies the data replicate earlier findings. First, with 500-ms exposure and 514-ms SOA, the high-anxious group showed a more positive attentional bias for threat-related words than the low-anxious group. A similar bias was observed for positive words, but only during the second task block. Similarly, Bradley, Mogg, White, Groom, and de Bono (1999) reported a positive bias for happy faces in generalized anxiety, that was limited to the second half of the task. They speculated that this effect might be due to mood regulating strategies that take time to build up. Second, consistent with Mathews et al. (1996) and Luecken et al. (2004), the high-anxious group showed a more positive bias for social-threat words with 14-ms exposure and 514-ms SOA than the low-anxious group. A similar, but non-significant, pattern was observed for physical-threat words.

The addition of two extra conditions enabled the independent comparison of effects of word-color onset asynchrony (SOA; 0 vs. $514 \mathrm{~ms}$ ) and exposure duration ( 14 vs. $500 \mathrm{~ms}$ ), yielding the time-course of attentional bias effects and the influence of processing time on these effects, respectively. The data revealed different tempo-

\footnotetext{
${ }^{46}$ The high-anxious group showed a (weak) positive bias in the 500/514, 500/0, and $14 / 0$ conditions $(t(21)=$ 1.6, $p=.14, t(21)=1.7, p=.11$, and $t(21)=1.6, p=.12$, respectively; $14 / 514: t(21)=-.06, p=.95)$. The lowanxious group had a (weak) negative bias in the $500 / 514$ condition, $t(24)=-1.5, p=.14$ (other conditions: .15 $<t(24)<.95, p>.36)$.
} 
ral characteristics for attentional bias effects triggered by physical-threat, socialthreat and positive words, which will be discussed successively.

When physical-threat words were presented for $500 \mathrm{~ms}$, allowing for processing the content of these words and permitting awareness, the high-anxious group showed a more positive bias than the low-anxious group. This was already evident at 0 -ms SOA and did not change for the 514-ms SOA. Physical-threat words presented for $14 \mathrm{~ms}$ did not lead to a bias. The more pronounced effects with 500-ms than with 14-ms exposure suggest that strategic processes, occurring after word meaning entered awareness, are crucial for anxiety-related attention bias for physical-threat words.

For social-threat words, in contrast to physical threat words, bias effects appeared to be larger with 14-ms than with 500-ms exposure. This suggests a counteracting influence by strategic processes, coming into play with longer processing time, on effects occurring at an early stage of processing. Initially, at an SOA of 0 $\mathrm{ms}$, both groups had a positive bias for social-threat words. After 514-ms, the highanxious group still had a positive bias, whereas the bias in the low-anxious group had reversed. This pattern is consistent with the idea that high-trait-anxious individuals take longer to disengage attention from the (location of) threat-relevant stimuli than low-anxious persons (e.g., Fox, 2004).

It should be noted that for social-threat words, bias effects were only observed during the first task block. One - speculative - explanation is that awareness varied over the course of the task and that the words presented for $14 \mathrm{~ms}$ became more visible during the second task block, allowing for strategic processes to operate (Mogg \& Bradley, 1999b). Alternatively, this block effect might be due to habituation, practice or changes in mood state. Further research is needed to examine the development of bias effects across task blocks.

An attentional bias toward positive words was observed in the high-anxious group during the second task block, if words were presented for $500 \mathrm{~ms}$, especially when bias was tapped at an SOA of $514 \mathrm{~ms}$. Since there were no bias effects with a 14-ms exposure, these findings suggest that strategic processes are crucial for an attentional bias for positive words (e.g., mood regulation or mood repair; Bradley et al., 1999).

Interestingly, for both physical and social threat words, given an SOA of $0 \mathrm{~ms}$, there was a large difference in bias between words presented for 14 and $500 \mathrm{~ms}$. This suggests that bias scores reflect the influence of processing time on attention well after probe onset. Apparently, bias scores do not purely reflect where attention is at the exact moment of probe onset, but are also influenced by word processing as it continues after that moment.

Differences in attentional bias observed for physical- and social-threat words may be related to their threat intensity level (cf. Wilson \& MacLeod, 2003) or current relevance (e.g., Fox, 1993a). Our participants were selected based on their 
scores on the Spielberger trait-anxiety inventory, measuring anxiety in social interaction situations, rather than responsiveness to physical-threat (Fox, 1993a; Eysenck, 1997). Furthermore, they were monitored by means of a video camera, and received performance feedback frequently. Therefore, it may be that our participants, and especially the high-anxious group, felt socially evaluated during the experiment, resulting in social stress. Thus, social-threat words were more related to the specific situation and current concerns than physical-threat words. Wilson and MacLeod (2003) found that attentional bias effects in high- and low-anxious people depend strongly on the threat intensity level of the stimuli. Future research should determine the temporal characteristics of biased attention for stimuli with different levels of threat intensity and relevance.

Inherent to an attentional bias task in which a probe is presented simultaneously with an emotional stimulus is that interference effects can come into play. It has been observed that high-anxious individuals are especially slow in color-naming words related to their worries or relevant threat (affective Stroop effect; see Williams, Mathews, \& MacLeod, 1996, for review). It may be argued that a similar interference effect contributed to our attentional bias scores at 0-ms SOA. However, Fox (1993b) observed comparable affective Stroop interference effects when the threatening word and color information were integrated and when they were spatially separated. If so, in our experiment, interference effects were similar when emotional word and probe (color) were spatially corresponding and when they were non-corresponding, resulting in a net zero effect on bias scores (non-corresponding corresponding).

To conclude, disentangling the effects of exposure duration and stimulus-probe SOA revealed specific temporal characteristics of attentional bias for various word types in a non-clinical sample. An anxiety-related bias for physical-threat words required a long presentation duration and was completely independent of SOA. In contrast, an initial bias toward shortly presented social-threat words in both highand low-anxious groups reversed in the low-anxious group to turn into a bias away from these words at a longer SOA. The latter result is consistent with reports of an anxiety-related difficulty in disengaging attention from threat-related stimuli (Fox, 2004). As a methodological note, for both physical- and social-threat words, a longer presentation duration affected the bias even if attention was already 'tapped' very early by the probe, suggesting that bias scores do not purely reflect where attention is at the moment of probe onset. Future research should determine the precise temporal characteristics of attentional bias to various threat stimuli in nonclinical and clinical anxiety/fear, in several situations. The resulting understanding of the cognitive mechanisms involved in anxiety may, in the end, help to improve clinical treatment. 



\section{Chapter 8}

General Discussion 
The focus of this dissertation was on preferential processing of emotionally relevant information in anxiety. In the first chapter, a review was given of the theoretical and empirical framework that has guided the research presented in this thesis. In addition, a novel approach was introduced, developed to investigate preferential processing of emotional information. The key idea underlying this new approach was that, if an emotional stimulus, that is presented together with a neutral stimulus, is preferentially processed, it may act as if it is presented alone. If so, it may yield a Simon effect: faster responses when emotional stimulus position corresponds with response position than when these positions do not correspond. Finding such a Simon effect for an emotional stimulus, presented simultaneously with a neutral one, would add evidence to the hypothesis of early preferential processing of the emotional stimulus. An important part of this dissertation project was dedicated to the elaboration and application of this new 'spatial affective Simon' approach (Chapters 3-6).

A second interest of this dissertation was in analyzing and mapping the temporal dynamics of anxiety-related attentional bias. In recent years, several researchers have taken an interest in this topic. They systematically manipulated exposure duration of the critical stimuli in dot-probe tasks, tracked eye movements or recorded event-related brain potentials (for a review, see Chapter 1, Section 1.2.2.2.2.). Still, little is known about the temporal characteristics of attentional bias in anxiety. In this dissertation, the temporal dynamics of selective attention in anxiety were studied in reaction-time paradigms that were similar to a typical probeclassification task, by analyzing reaction-time distributions (Chapter 6) and by independently manipulating stimulus-probe onset asynchrony and stimulus duration (Chapter 7).

In this final chapter, the main findings of the studies reported are first briefly discussed, followed by some issues in interpretation and methodology. Recommendations and suggestions for further research are given. Finally, some outstanding questions and 'hot' issues in research on preferential processing in anxiety today are described and ideas for future research are presented.

\subsection{Review and Discussion of the Empirical Findings}

The structure of this section is organized around the two main topics addressed in this dissertation: (1) spatial affective Simon effects and (2) temporal dynamics of selective attention in anxiety. 


\subsubsection{Spatial Affective Simon Effects}

The Spatial Affective Simon Task (SAST) was introduced as a measure of early preferential processing of emotionally relevant information. During this task, as applied in Chapters 3-5, one emotional and one neutral word were visually presented, one above the other, both in red or green, and participants responded to these colors by pressing the upper or lower key on a response box, respectively. Crucially, the spatial correspondence of emotional word and response was systematically varied across trials. Spatial affective Simon scores were computed by subtracting mean reaction time (RT) when emotional stimulus and response spatially corresponded from mean RT when they did not correspond. So, positive scores reflected faster responses on corresponding trials, whereas negative scores reflected faster responses on non-corresponding trials.

The SAST was applied to study preferential processing of threat-relevant information in undergraduates exposed to a physical stressor (Chapter 3), in high-traitanxious undergraduates (Chapter 4), and in an anxious clinical sample (Chapter 5). In all three studies, masked word pairs were presented for 14 or $500 \mathrm{~ms}$ (intermixed). The 14-ms condition was included to investigate whether anxiety-related preferential processing occurs under conditions of restricted awareness. With 500ms exposure, there was more time for stimulus processing, allowing later controlling strategies to come into play.

In the SAST experiment in Chapter 3, with 500-ms exposure, undergraduates exposed to height showed a positive Simon score for physical-threat words, whereas undergraduates tested in a lab showed a negative score. A similar, but nonsignificant, pattern was observed for height words. The opposite pattern was obtained for social-threat words, with the control group showing a positive score, and the height group a non-significant negative score. With 14-ms exposure, there were no significant effects.

In the SAST experiment in Chapter 4, with 14-ms exposure, high-trait-anxious undergraduates showed a positive Simon score for social-threat words, no significant effect for physical-threat words, and a negative score for positive words. Low-traitanxious undergraduates showed no effect at all. With 500-ms exposure, the group differences tended to reverse, with high-trait anxious, compared to low-anxious, undergraduates showing a negative score for social-threat words and a positive score for positive words, but these effects were weak.

In the SAST experiment in Chapter 5, during the second half of the task, anxious patients showed a positive Simon score for social-threat words, no significant effect for physical-threat words, and also a positive score for positive words, irrespective of exposure duration. Matched, non-psychiatric controls showed, if anything, the opposite pattern. During the first half of the task, there were no significant effects. 
In a fourth experiment (Chapter 6), spatial affective Simon effects were not studied in a SAST, but in a probe-classification task in which the correspondences of emotional word, probe, and response positions were varied independently. Again, one emotional and one neutral word were presented, one above the other, for 14 or $500 \mathrm{~ms}$ (intermixed and masked). Here, the pair was printed in white and followed by a red or green dot-probe, taking the position of one of the words. Response position was again determined by the color. As in the SAST, the spatial correspondence of emotional stimulus and response varied over trials, making it possible to study spatial affective Simon effects. It was explained that the probe classification task differed in two important aspects from the SAST (see also Chapter 1, Section 1.3.3.2.): (1) Responses were given to a probe appearing after word offset, and were, therefore, delayed compared to the emotional stimulus. As a result, bias effects were tapped later in time than in the SAST as used in the previous studies, in which probe information appeared at word onset; (2) Relevant probe information appeared at only one of the locations. It was argued that this probe classification task can be used to study both spatial affective Simon effect, called 'response bias' in Chapter 6, and attentional bias (reflected in the effects of spatial correspondence of emotional stimulus and probe). The study in Chapter 6 aimed to investigate (1) whether similar response bias effects could be observed with the probe classification task as with the SAST in Chapter 4, despite the differences between the paradigms, and (2) how response bias effects in the probe classification task relate to attentional bias effects in the same task. High-trait-anxious undergraduates showed a positive Simon score for social-threat words, irrespective of exposure duration, and no effects for physical-threat or positive words. Low-trait-anxious undergraduates showed only a weak negative score for physical-threat words with 500-ms exposure; the group difference did not reach significance. Response bias for social-threat words was opposite to attentional bias.

To summarize, most importantly, in all four studies, positive spatial affective Simon scores were observed for threat-relevant words in anxious individuals and individuals exposed to a stressor. Non-anxious or low-anxious controls showed no significant effect or the opposite effect. The effects were content-specific in the sense that no or opposite Simon scores were obtained for positive words or for threatwords that were not related to current concerns or the current situation. Only the patient group (Chapter 5) showed the same pattern for positive and threat-relevant words, at least in the second half of the task. Speculative explanations were given for their positive score for positive words: (1) strategies to maintain positive mood, resulting in biased processing of positive information, (2) high arousal level of positive and social-threat words compared to physical-threat words, and (3) positive words related to social situations or being antonyms of social-threat words may have a threatening meaning for anxious patients. 
Simon scores deviating from zero reflect the existence of spatial affective Simon effects, also called response bias. The present evidence for anxiety-related response bias is interesting and relevant for theories on biased processing associated with anxiety. In this dissertation, several explanations were suggested to account for the observed response bias effects. These effects can, for example, be attributed to extreme enhanced processing of the emotional stimulus or to later biased processing towards or away from the emotional stimulus. The proposed explanations are further discussed in Section 8.2.1.

Different effects of exposure duration (processing time) were observed in the four experiments. These differences were interpreted in terms of the different anxiety groups under consideration and the procedural differences between the studies. Differences might, for example, be due to a different type of stress / anxiety (physical stressor (height) in Chapter 3 vs. social anxiety (STAI-trait) in Chapter 4) and to differences between sub-clinical and clinical samples in their ability to counteract immediate bias effects (Chapter 4 vs. Chapter 5). These accounts were based on previous observations and interpretations in the Stroop- and dot-probe literature. The weak or absent effects with 14-ms exposure in the height study (Chapter 3) might also be due to the fact that the daylight on the bridge and the bright lamps in the lab might have reduced the visibility of the stimuli. All other studies took place in a dimly lit room. Finally, in the probe-classification task used in Chapter 6, the effects were always 'tapped' after a delay, after stimulus offset. That is, with 14-ms and 500-ms exposure, the probe appeared $28 \mathrm{~ms}$ and $514 \mathrm{~ms}$ after word onset, respectively. In the SAST, with both exposure durations, the probing information appeared at word onset. So, differences between Simon scores as observed in Chapter 4 (SAST) and in Chapter 6 (probe-classification task) might be explained by differences in the moment of tapping. However, as explained before, moment of tapping was not the only difference between the two tasks. Chapter 7 described an experiment in which anxiety-related bias effects were measured in a task similar to the probe-classification task described before, but during which bias effects were not only tapped after a delay, but also at word onset as in the SAST (see also Section 8.1.2.2.).

Finally, the finding of opposite response bias and attentional bias effects for social-threat words in Chapter 6 was taken to suggest that these bias effects do not simply reflect the same single mechanism. Furthermore, to purely measure attentional bias, we recommended to apply probe-classification or probe-detection tasks, rather than probe-position tasks. In typical probe-position tasks, there is a direct spatial correspondence between emotional stimulus, probe and response. Therefore, it was suspected that in these tasks, response bias could contribute to attentional bias. 
The main conclusions of the present studies on spatial affective Simon effects (response bias) can be summarized as follows:

1. This first series of experiments showed that emotionally relevant words when presented together with a neutral word can yield a Simon effect, even under conditions of restricted stimulus awareness.

2. The observed bias effects were largely specific to current (threat) concerns and to the situation.

3. These data provide additional evidence for biased processing of emotionally relevant stimuli in anxiety, by applying the novel approach.

4. The SAST seems to be a promising tool to investigate preferential processing of emotional information, and may be useful in further research to acquire elaborate knowledge of the mechanisms associated with emotions and psychiatric disorders.

5. These first studies differed on a number of variables, and, of course, further studies are needed in which potentially important variables are systematically manipulated.

\subsubsection{Temporal Dynamics of Selective Attention in Anxiety}

\subsubsection{Distributional RT Analyses}

In Chapter 6, it was argued that distributional analyses of behavioral response latencies in a dot-probe task might inform us about the time-course of the hypothesized anxiety-related bias effects. Relatively fast responses to the probe might be more sensitive to early bias effects, and relatively slow responses might be more influenced by later cognitive processes. Distributions of the RTs obtained during the probe-classification experiment described in the previous section were analyzed. Positive attentional bias scores (faster responses when emotional stimulus and probe correspond than when not) were taken to reflect selective attention to the emotional stimulus.

Overall, in line with previous research, high-anxious, compared to low-anxious, undergraduates showed a positive attentional bias score for physical-threat words, with 14-ms exposure (limited to the first half of the task) and with 500-ms exposure. Interestingly, the group difference observed with 14-ms exposure increased as a function of response time. Whereas the positive bias of the high-anxious group did not vary with different RTs, the bias of the low-anxious group became more negative with longer RTs. With 500-ms exposure, the group difference did not depend 
on response speed. These results were explained in terms of delayed attentional disengagement from threat in trait-anxiety (cf. Fox, 2004), and are consistent with previous results from exogenous cueing studies and dot-probe studies (Chapter 1, Section 1.2.2.2.1.). In several (single cue) exogenous cueing studies, it has been found that responses of anxious individuals are especially slow when a threat-related stimulus is followed by a probe at the other location, compared to when a positive or neutral stimulus is presented (e.g., Fox, Russo, Bowles, \& Dutton, 2001; Yiend $\&$ Mathews, 2001). These findings have been taken to indicate delayed disengagement from threat in anxiety. In dot-probe studies, it has been found that responses are slower when the threatening stimulus in the stimulus pair and the probe do not spatially correspond than when two neutral stimuli are presented. In contrast, no differences have been found between responses when threatening stimulus and probe correspond and responses when two neutral stimuli are presented (e.g., Koster, Crombez, Verschuere, \& De Houwer, 2004). Also this pattern has been taken to indicate delayed disengagement from threat.

High-anxious individuals also showed a rather weak negative attentional bias for social-threat words, irrespective of exposure duration (first half of the task), and a weak positive attentional bias for positive words with 500-ms exposure (second half). These effects and also the observed response bias effects, as summarized in Section 8.1.1., did not vary with response speed.

To conclude, the finding that at least some dot-probe effects depend on response speed suggests that distributional RT analyses might inform us about the temporal dynamics of the hypothesized biases. This finding has some important practical implications, as outlined in Chapter 6.

\subsubsection{Time-Course of Attentional Bias and Influence of Processing Time}

In the experiment in Chapter 7, the time-course of anxiety-related attentional bias effects and the influence of processing time on these effects were examined. Hightrait-anxious and low-trait-anxious undergraduates performed a task that was similar to the classification task described before (Chapter 6). Again, one emotional and one neutral word were presented, one above the other, for 14 or $500 \mathrm{~ms}$ (intermixed and masked). But, here, relevant probe information (i.e. color) appeared at word onset or after $514 \mathrm{~ms}$. By independently manipulating word duration and wordcolor onset asynchrony (SOA), it was possible to experimentally dissociate the effects of processing time and moment of tapping.

In the two conditions that resembled previous dot-probe studies (14-ms or 500ms exposure combined with 514-ms SOA), the data replicated earlier findings (e.g., Mogg \& Bradley, 1999; Bradley, Mogg, White, Groom, \& de Bono, 1999; Mathews, Ridgeway, \& Williamson, 1996; Luecken, Tartaro, \& Appelhans, 2004). Importantly, disentangling the effects of exposure duration and SOA revealed 


\section{Chapter 8}

specific temporal characteristics of attentional bias effects for (1) physical-threat, (2) social-threat (first half of the task) and (3) positive words (second half). (1) An anxiety-related bias for physical-threat words required 500-ms exposure duration and was completely independent of SOA. The more pronounced effects with 500$\mathrm{ms}$ than with 14-ms exposure were taken to suggest that strategic processes were crucial for the bias for physical-threat words. (2) An initial positive bias for socialthreat words with 14-ms exposure in both high- and low-anxious groups reversed in the low-anxious group to turn into a negative bias at 514-ms SOA. This result was explained in terms of delayed attentional disengagement from social-threat in anxiety (e.g., Fox, 2004), and was in line with some previous results from exogenous cueing and dot-probe tasks (see above Section 8.1.2.1., and Chapter 1, Section 1.2.2.2.1.). The larger effects with $14-\mathrm{ms}$ than with 500 -ms exposure were taken to suggest a counteracting influence of later strategic processes. (3) The high-anxious group showed a positive attentional bias for positive words with 500-ms exposure, especially with 514-ms SOA. The lack of a significant effect with 14-ms exposure was taken to suggest that strategic processes were crucial for the bias for positive words (e.g, mood regulation). Finally, the large difference in bias between threat words presented for 14 and $500 \mathrm{~ms}$, given 0 -ms SOA, suggests that bias scores reflect the influence of processing time on attention well after probe onset.

In the majority of previous dot-probe studies, exposure duration and SOA have been confounded. Did these studies draw incorrect conclusions? In general, previous dot-probe studies during which the probe immediately followed the stimulus pair have concluded that high-anxious, relative to low-anxious, individuals show a content-specific attentional bias for mildly threatening stimuli with 500 -ms exposure, and even with 14-ms exposure (masked). The results in Chapter 7 suggest that these conclusions are correct, but incomplete. To allow for an accurate view of anxiety-related attentional bias elicited by stimuli presented for, for example, 14 or $500 \mathrm{~ms}$, the temporal course of these effects should be measured by systematically manipulating SOA across a broad range of intervals, while keeping exposure duration constant. Similarly, to fully understand the true effects of processing time, exposure duration should be systematically varied, while keeping SOA constant.

\subsubsection{Main Conclusions Temporal Dynamics}

The main conclusions of the present studies on the temporal aspects of anxietyrelated attentional bias can be summarized as follows:

1. Distributional analyses of RTs on a dot-probe task may help to reveal the timecourse of attentional bias 
2. Disentangling the effects of word duration (processing time) and word-probe onset asynchrony (moment of tapping) revealed specific temporal characteristics of attentional bias for various word types in a non-clinical sample.

3. The data suggested that high levels of trait anxiety are associated with delayed disengagement of attention from threat.

4. These studies were the first in which these analyses and manipulations were applied in the attentional bias literature. Further research is, of course, needed to replicate and generalize the current findings.

\subsection{Issues in Interpretation}

This section deals with two issues in interpretation, raised throughout this thesis: (1) the interpretation of spatial affective Simon effects and (2) the interpretation of the observed differences between task blocks.

\subsubsection{Interpretation of Spatial Affective Simon Effects}

In Chapters 3-6, several explanations were suggested to account for the observed spatial affective Simon effects (response bias), as reflected in positive and negative Simon scores in the SAST or probe classification task. At this stage, it is not clear which processes exactly underlie these effects. Note that this is also true for attentional bias effects as measured in the dot-probe task (attentional shift vs. disengagement; e.g., Fox, 2004), classical Stroop interference effects (e.g., processes at the stage of response selection and/or perceptual encoding; de Houwer, 2003; MacLeod, 1991), emotional Stroop effects (e.g., selective attention, cognitive avoidance, disengagement, or response inhibition; de Ruiter \& Brosschot, 1994; Fox, 2004; Williams, Mathews, \& MacLeod, 1996), and classical Simon effects (e.g., processes at level of response selection or stimulus identification: Hasbroucq \& Guiard, 1991; Kornblum, Hasbroucq, \& Osman, 1990; temporal overlap of cognitive representations of stimulus and response: Hommel, 1993b). In the following paragraphs, the alternative explanations for spatial affective Simon effects suggested throughout this thesis are discussed. In the remainder of this section, 'spatial affective Simon effect' refers to the effect as reflected in positive spatial affective Simon scores. Effects reflected in negative scores are referred to as 'reverse spatial affective Simon effect'.

The original explanation for spatial affective Simon effects is that the emotional stimulus is preferentially processed at a very early stage of processing, and pops out 


\section{Chapter 8}

of the display. It may act as if presented alone and elicit a classical Simon effect: faster responses when the irrelevant position of this stimulus corresponds with response position than when they do not correspond. However, reverse spatial affective Simon effects, as for example observed in Chapter 3 for the control group for social-threat words and in Chapter 4 for the high-trait-anxious group for positive words, are difficult to reconcile with this account. Such effects would imply that the neutral stimulus that was paired to the emotional one was automatically processed at a very early, pre-attentive stage of processing.

A related account for spatial affective Simon effects is that the emotional stimulus receives more efficient, enhanced processing, relative to the accompanying stimulus (i.e., attentional bias) and yields a classical Simon effect, although it does not pop out in the strict sense. Following this interpretation, reverse spatial affective Simon effects might reflect enhanced processing of the neutral stimulus, compared to the emotional stimulus, the processing of which might be suppressed. According to this account, an attentional bias towards the emotional stimulus results in a spatial affective Simon effect, whereas an attentional bias away from the emotional stimulus results in a reverse spatial affective Simon effect. In the SAST, an attentional shift by itself should not lead to an increased Simon score, because both stimuli convey the relevant color information. The SAST measures response bias; attentional bias can only influence the scores to the extent that it occurs together with a genuine response bias. The probe classification study reported in Chapter 6 revealed a dissociation between response bias and attentional bias effects, suggesting that these effects are not a reflection of the same single underlying mechanism. However, as already mentioned, there are several structural differences between the probe classification task and the SAST, and therefore there also exist differences between the response bias effects measured by these tasks.

The effects can also be attributed to a behavioral tendency to approach or avoid the emotional stimulus. That is, spatial affective Simon effects might reflect an early tendency to move towards the emotional stimulus, whereas the reverse effects might reflect a tendency to move away from it (and to approach the neutral stimulus paired to it). In the literature, there is a general notion that emotionally and motivationally salient stimuli can prompt or facilitate response (action) tendencies. Negative situations or stimuli (except in 'anger': see Harmon-Jones, 2003) have usually been related to behavioral inhibition, avoidance and escape tendencies, while positive situations or stimuli have been related to an approach response (e.g., Frijda, 1986; Lang, Davis, \& Öhman, 2000, Gray \& McNaughton, 2003; Neumann, Förster, \& Strack, 2003). From an evolutionary perspective, the key function of anxiety is rapid detection of potential threat, so as to respond efficiently and adaptively when necessary (e.g., Oatley \& Johnson-Laird, 1987; Öhman, 1993). Mogg and Bradley $(1998,2004)$ conceptualize anxiety as an aversive motivational state, associated with an early bias in the evaluation of threat, resulting in tendencies that 
interrupt current goals and activities. Such tendencies include cognitive processes (e.g., attentional disengagement and avoidance), physiological responses (e.g., heart rate) and also action tendencies (i.e., readiness to engage in defensive avoidant behavior, like freezing or escape). Mogg and Bradley's model implies that action tendencies depend on early stimulus evaluation, with further attentional processing not necessarily being involved.

Evidence for response (action) tendencies in emotion comes, for example, from psychophysiological research showing that threatening stimuli can modulate defensive startle eye blink reflexes (Lang et al., 2000) and that emotional stimuli can increase activity and excitability of motor areas of the brain (Hajcak et al., 2007). Furthermore, behavioral research has revealed that negative information may inhibit response execution (Derryberry, 1991). In addition, participants have been found to be faster to push a lever (or release a button) when a negative than when a positive stimulus was presented, irrespective of whether stimulus valence was task relevant. In contrast, they were faster to pull (or press a button) when a positive rather than a negative stimulus was presented in such a set-up (e.g., Solarz, 1960; Sobotka, Davidson, \& Senulis, 1992; Chen \& Bargh, 1999; Wentura, Rothermund, \& Bak, 2000; Rotteveel \& Phaf, 2004; Rinck \& Becker, in press). As well, stimuli presented during arm flexion (pressing upward) have been evaluated more positively than stimuli presented during arm extension (pressing downward; Cacioppo, Priester, \& Berntson, 1993). Pulling, pressing a button, and arm flexion have been associated with the tendency to approach, while pushing, releasing a response button, and arm extension with avoidance. ${ }^{47}$ Importantly, in the behavioral approach/avoidance studies cited, the affective correspondence of stimulus and response was manipulated. The SAST can be seen as a measure of response tendencies towards or away from emotional stimuli.

The finding of spatial affective Simon effects for threat-relevant stimuli in all four studies makes an interpretation solely in terms of response tendencies unlikely. In light of the general view that negative stimuli elicit an avoidance or withdrawal response, it is implausible to assume that in these studies threat-relevant stimuli elicited an approach reaction. It could, however, be that the scores arise from a combination of an effect caused by early preferential processing and an effect caused by a response tendency. In anxious individuals, a positive score caused by early, preferential processing of threat-relevant information might be reduced by a negative score caused by some immediate avoidance of these stimuli. The negative score observed in the control group in Chapter 3 for physical-threat words might indi-

\footnotetext{
${ }^{47}$ Pull and push responses are ambiguous since their interpretation depends on the point of reference, that is the external stimulus or the participant himself (e.g., Neumann et al., 2003; Markman \& Brendl, 2005; Rinck $\&$ Becker, in press).
} 
rectly reflect a relative dominance in all individuals to avoid these threat-related stimuli.

Finally, it could be that response tendencies invariably follow shifts in attention. Several researchers have suggested a close link between spatial attention and manual responses (Allport, 1987; Neumann, 1990; Nicoletti \& Umiltà, 1989; Rizzolatti, Riggio, \& Sheliga, 1994). Consider the possibility that when attention shifts towards a certain stimulus, motor responses in the direction of this stimulus are also prepared. If so, positive and negative Simon scores might indirectly reflect an attentional bias towards and away from the emotional stimulus, respectively.

Further research is needed to resolve which processes underlie the spatial affective Simon effects. For instance, one way to examine whether SAST effects reflect dominant response tendencies and / or preferential attentional selection is to measure event-related brain potentials on-line during SAST performance. The stimuluslocked Lateralized Readiness Potential is assumed to be related to selective activation of motor responses (De Jong, Wierda, Mulder, \& Mulder, 1988; Gratton, Coles, Sirevaag, Eriksen, \& Donchin, 1988; De Jong et al., 1994, Exp. 4), and the N2pc component is assumed to reflect spatially selective attentional processing, (Luck \& Hillyard, 1994; Eimer, 1996; Woodman \& Luck, 1999; fearful faces: Eimer \& Kiss, 2007). It can be argued that EEG recording during the performance of a horizontal SAST (i.e., left/right stimulus and response positions) can map both selective response and attentional bias effects, as reflected by LRP and N2pc components, respectively. In a vertical SAST, attention-related activity is apparently symmetrical between the hemispheres and therefore invisible in the lateralized ERPs (cf. De Jong et al., 1994, Exp. 4). Therefore, if ERP modulation is only found in a horizontal and not in a vertical SAST, this would mean that the SAST effect reflects an attentional selection process rather than a response tendency. ${ }^{48}$

\subsubsection{Interpretation of Block Effects}

In all studies conducted for this dissertation, participants completed two task blocks, separated by a break of a few minutes. ${ }^{49}$ The blocks always consisted of the same stimulus pairs, the same conditions and the same number of trials. They did only differ in random trial order, and random allocation of stimulus pairs to conditions.

\footnotetext{
${ }^{48}$ In fact, we (Schrooten \& Smulders) have already set up an ERP experiment during which high-trait-anxious undergraduates performed a vertical as well as a horizontal SAST and classical Simon task. At the moment of this writing, this study is still in progress.

${ }^{49}$ The tasks reported in Chapters 4 and 6 were performed during the same experimental session. The presentation order of the four task blocks was balanced within anxiety groups in the following way: abba or baab.
} 
In three out of the five studies reported, there were significant differences between the blocks in the relevant effects. In the experiments on spatial affective Simon effects, only in the clinical study, there was a significant block effect with the patient group showing positive Simon scores for social-threat and positive words during the second block, whereas there were no significant effects during the first block. In both probe-classification studies, for social-threat words, there were only attentional bias effects during the first block and for positive words only during the second block. In addition, in Chapter 6, attentional bias effects for physical-threat words presented for 14-ms were significant during the first, but not during the second block.

One explanation for the observed block effects is that genuine anxiety-related bias effects may develop over the course of the task. In this thesis, changes in bias effects over the course of the task were interpreted in terms of strategic influences (e.g., mood regulation), changes in arousal or mood, habituation to the situation or to the emotional stimulus material, and changes in visibility and awareness with 14ms exposure. It was also argued that some bias effects may only become apparent after more automatized performance on the primary task. At this point, these interpretations remain speculative and await further research. For instance, it would be valuable to systematically measure arousal, emotional state, and stimulus awareness throughout the session, and to study the effects of repeated exposure of emotional stimuli (cf. Liu, Qian, Zhou, \& Wang, 2006). Future research needs to determine whether the observed block effects are replicable in studies with similar participants, under similar conditions, and with the same task parameters as in the study being replicated.

Further research is required to confirm that the block effects were not just a reflection of internally inconsistent, unreliable results. On the basis of his observations in various dot-probe tasks that there is no (or low) correlation between attentional bias scores obtained from two random task halves, between scores from quadruplets consisting of one exemplar of each condition, and between scores measured at different test occasions separated by a week, Schmukle (2005) concluded that the dot-probe task is no reliable measure of attentional bias, at least in a non-clinical anxious population. In his paper, Schmukle overlooked the possibility that bias scores may systematically change over the course of the task. As said before, also this possibility has to be investigated in further research, both for the dot-probe task and the SAST. 


\subsection{Issues in Methodology}

This section deals with some methodological issues: the particular kind of stimulus material used and the specific design of the tasks applied in this dissertation, respectively.

\subsubsection{Stimulus Material}

In all studies reported in this dissertation, verbal stimuli were used. The physicalthreat, social-threat and positive words were mostly taken from previously published lists (especially Mathews \& MacLeod, 1985; MacLeod, Mathews, \& Tata, 1986). A large pool of physical-threat-neutral, social-threat-neutral, positive-neutral, and neutral-neutral pairs was created. The final pairs were selected based on ratings by independent, native Dutch, staff members. Physical-threat words, social-threat words, and neutral words paired with them were rated as to whether they were associated with physical-threat and social-threat, respectively. For positive words, neutral words paired with them, and filler words, the valence was rated. It could be valuable for further research to let participants themselves rate the stimuli, at the end of the experimental session, and to include also other stimulus characteristics, such as threat-intensity level, current relevance, subjective frequency (familiarity), and arousal. This information might help to get a better understanding of the content specificity observed in the data. In addition, future studies might extend this work with verbal stimulus material by using other kinds of stimuli.

Pictures do depict the source of potential threat in a more direct, concrete way than words. So, from an evolutionary perspective, they can be seen as being more ecologically valid. In previous dot-probe research, pictures have been used of angry or fearful facial expressions, spiders, snakes, and threat-related scenes (for a review, see Chapter 1). It has been suggested that the greater the evolutionary relevance or the more intense the threat intensity level of the stimuli, the more likely it is that also low-trait-anxious individuals preferentially process these stimuli (Mogg, McNamara, et al., 2000; Wilson \& MacLeod, 2003; cognitive models by Mogg \& Bradley, 1998; Mathews \& Mackintosh, 1998; for a review, see Chapter 1).

The SAST as used in the present dissertation can be easily modified to study spatial affective Simon effects for pictorial stimuli. Remember that the three elements of critical importance in the SAST are that (1) the emotional stimulus is presented together with a neutral one, (2) the relevant information appears simultaneously at both stimulus locations, and (3) the spatial correspondence of emotional stimulus and response varies systematically across trials. One suggestion for a pictorial SAST is to present two pictures, side by side, with one vertical bar on the left side of the left picture and another bar on the right side of the right picture, and to 
let participants respond to the color of these bars by pressing a left or right key. ${ }^{50}$ One may also think of versions during which the probing information is more integrated with the pictures.

The content of threat-related pictures is, just like the meaning of threat words, only semantically related to threat. Participants know that these stimuli themselves do not pose or signal any real risk. It would be interesting to study anxiety-related bias effects in a situation during which threat can be really expected and during which the stimuli function as predictors for risk or safety. For example, one may pair stimuli, during the experimental session, with the occurrence of a negative outcome or with the occurrence of a positive outcome (or absence of a negative outcome).

An additional advantage of such conditioned stimuli over intrinsically emotional stimuli is that one can perfectly control their emotional as well as perceptual content. Stimuli can be used that have an equivalent intrinsically emotional valence at the outset of the experiment (e.g., Armony \& Dolan, 2002) and stimuli with highly controlled physical content (e.g., Baas, Kenemans, Bőcker, \& Verbaten, 2002). Furthermore, one can counterbalance across participants which stimulus will be associated with the aversive event. Good control of physical stimulus characteristics is advantageous in emotion research, for it is important that there is no systematic confound between emotional content and some other stimulus features. Furthermore, in research on selective processing of emotionally relevant information, the critical stimulus is presented together with at least one other stimulus, and, for a sound interpretation of the results, it is essential that the stimuli only systematically

\footnotetext{
${ }^{50}$ Actually, this version of the SAST was used in an undergraduate laboratory class (Schrooten \& Smulders). Thirty-nine unselected undergraduates performed the task, during which spider pictures, negative, positive, and neutral pictures, paired to neutral pictures, were presented for $650 \mathrm{~ms}$. Another difference with the SAST experiments reported before was that the computer monitor was tilted, with the screen pointing upwards, and that the response buttons were stuck on the screen, just below each stimulus position. All participants completed a practice block of 24 trials, followed by two balanced blocks of 192 experimental trials. Other task parameters and the procedure were the same as in Chapter 4. Briefly, the expected positive spatial Simon score for evolutionary relevant stimuli (i.e., spiders) in the entire sample was not significant. However, there was some positive correlation between self-reported trait-anxiety scores and Simon scores for spiders, in the second half of the task (Spearman $r=.30, p$ (1-tailed) $=.03$; Pearson's $\rho=.27, p(1$-tailed $)=.05)$. This finding is in line with previous findings showing a more positive Simon score in anxious, compared to low-anxious, individuals for threat-related stimuli. The lack of the expected correlation between Simon scores for spiders and self-reported spider fear, as measured by the Fear of Spiders Questionnaire (FSQ; Muris \& Merckelbach, 1996), might be due to the low FSQ scores in the sample. Only two participants scored higher than 50, the minimum score for being considered as having high fear for spiders (Szymanski \& O’Donohue, 1995). The lack of significant results for positive or negative pictures might be due to stimulus selection, with having a too heterogeneous set of pictures. Importantly, converting the abstract spatial correspondence of emotional stimulus and response into a more direct relationship might have influenced any behavioral approach / avoidance tendencies that might contribute to spatial affective Simon effects. Further research is needed to resolve this issue (see also Section 8.2.1.).
} 
differ in emotional content, and not also in other features. In the studies conducted for this dissertation, as in previous dot-probe studies, the words were matched in terms of number of letters (non-proportional font) and objective frequency (as documented in a large database). It will be much more difficult to match photographs in terms of perceptual features or frequency (see also Chapter 1, Section 1.2.2.1.). By using simple, basic stimuli (e.g., gratings, color patches) one can overcome difficulties in matching. Finally, it is in principle always possible that intrinsically emotional stimuli may have some personal meanings depending on individual experiences, or that different responses to these stimuli may have been well-rehearsed already (Mackintosh \& Mathews, 2003; Stormark \& Hugdahl, 1996). This problem can be overcome by controlling the emotional content of unfamiliar (neutral) stimuli by associating them with a negative or positive meaning during the experiment.

In attentional bias literature, a few studies have been reported during which such predicting stimuli have been used. Derryberry and Reed (2002), for example, presented during an exogenous cueing task arrow cues that predicted the loss or gain of points, depending on response speed. Situations where a negative outcome (loss) was expected, were seen as threatening, whereas those where a positive outcome (gain) was expected were seen as safe. Others used fear-conditioned stimuli that predicted the occurrence of a physically nocuous stimulus, such as a burst of white noise (exogenous cueing: Stormark \& Hugdahl, 1996; Stormark, Hugdahl, \& Posner, 1999; Koster, Crombez, Van Damme, Verschuere, \& De Houwer, 2004; dot-probe: Armony \& Dolan, 2002). Future research should further investigate the nature of biased processing of stimuli that have acquired an aversive value through conditioning and stimuli predicting some real risk, for example by using the SAST and the dot-probe task. ${ }^{51}$

\footnotetext{
${ }^{51}$ In an undergraduate laboratory class, a first attempt was made to study spatial affective Simon effects for fear-conditioned stimuli by using the SAST (Schrooten, Jongen, \& Smulders). Two simple geometric figures with the same size were presented, side by side, both in red or green, and participants responded to these colors by pressing the right or left key on a response box, respectively. Following Armony and Dolan (2002; see also Chapter 1, Table 1.1), acquisition trials and test trials were intermixed, in order to prevent extinction of conditioning. During acquisition trials, two squares or rhombi were presented for $250 \mathrm{~ms}$. Fifty milliseconds after the onset of this pair, an aversive or neutral tone was delivered for $200 \mathrm{~ms}$ through a headphone. The aversive tone was a burst of white noise at $100 \mathrm{~dB}$; the neutral tone a $1000 \mathrm{~Hz}$ stimulus at $71 \mathrm{~dB}$. Which geometrical figure, irrespective of color, was followed by an aversive or neutral tone was counterbalanced across participants. Participants were informed as to which compound pair would signal white noise, and which pair the neutral tone. During test trials, one square and one rhombus were presented for $250 \mathrm{~ms}$, never paired with a tone. Finally, also filler trials were included, during which two triangles (both pointing upward or downward, or one upward and one downward) were presented for $250 \mathrm{~ms}$, never followed by a tone. Sixty participants completed a practice block of 36 trials, followed by three balanced blocks of 128 trials (same number of trials per condition). Briefly, participants rated white noise, the compound pair associated with this noise, and the single figure making up this pair as more unpleasant than the neutral tone, the compound pair associated with this neutral tone, and the single figure making up this pair, respectively. However, no significant affective
} 


\subsubsection{SAST Design}

The very attentive reader may have noticed that in the SAST the fixation cross also changed color from white to the word color. It was decided to give the fixation cross the color of the words and to leave this cross (and the color information) on the screen until a response was given, because, if not, it was very difficult to perform the task in the 14-ms exposure condition (14-ms colored words + 14-ms colored mask). Therefore, it might be the case that participants adopted the strategy to base their responses on the color of the fixation cross, and the words might be ignored. So, although the instruction was to determine the color of the word pair, the (color of the) words might be irrelevant for the task. It can be argued that attentional focus was measured, rather than selective processing effects. However, the results of the three SAST experiments reported show that color discrimination latencies were influenced by the spatial correspondence of emotionally relevant word and response (spatial affective Simon effect). So, even when the fixation cross was colored, and therefore the words might have been ignored, the meaning of some critical words have yielded a significant Simon effect. Note that also in a typical dot-probe task, during which responses are given to a probe following the stimulus pair, emotional stimuli are task-irrelevant, and can be ignored. It is exactly the fact that they nevertheless have an effect which is interesting. Finally, it can be that, when the cross does not change color and the words become more task-relevant, the effects will be larger than observed in the present experiments. In addition, individuals may differ in how well they can fixate on the cross (cf. attentional control, Derryberry \& Reed, 2002) and it may be the case that smaller or no effects will be observed in participants with good attentional control. It would be interesting to address these topics in future research.

\footnotetext{
Simon effects were obtained for the fear-conditioned stimuli. In Armony and Dolan's dot-probe study, angry faces were used as to be conditioned stimuli. Based on Seligman's (1971) "preparedness theory" of fear learning, it might be expected that it is easier to learn associations between aversive stimuli and stimuli that were potentially threatening for our ancestors, than between aversive stimuli and evolutionary irrelevant stimuli, such as the figures used in the present SAST. Furthermore half of the stimuli associated with aversive noise were red, the other half green, and the same was true for stimuli associated with a neutral tone. The choice of red and green as relevant stimulus information was in parallel with the first SAST experiments with words, but might be, in retrospect, not optimal in the present context in that there are already well-learned associations between these colors and danger/safety. Furthermore, it might be that the square and the rhomb were too difficult to discriminate. Finally, only subjective ratings of pleasantness were included. It would be valuable for further research also to incorporate more objective, psychophysiological measures of fear.
} 


\subsubsection{Attentional Bias and Response Bias Measured in a Single Probe- Classification Task}

In Chapter 6, a probe-classification task was used during which positions of the emotional words, probes and responses varied along the same dimension (vertically) and during which these positions were manipulated in an orthogonal manner. As illustrated in Table 8.1., this task has a rather complex structure, including three important contrasts: (1) the spatial correspondence of emotional stimulus and probe (attentional bias), (2) the spatial correspondence of emotional stimulus and response (spatial affective Simon effect or response bias), and (3) the spatial correspondence of probe and response (classical Simon effect for the probe). It was argued that this task can be used to study both attentional bias and response bias (and, of less interest for now, classical Simon effects).

A critical reader may have noticed that it was tacitly assumed that there was no interaction between attentional bias and response bias, or between each of these biases and the classical Simon effect. After all, in the task used, any interaction between attentional bias and response bias is indistinguishable from the classical Simon effect. Likewise, any interaction between attentional bias and classical Simon effect was confounded with response bias, and any interaction between response bias and classical Simon effect was confounded with attentional bias. So, it was not possible to determine the independence of the separate bias effects.

Importantly, in a probe-classification task, the main effects of attentional bias, response bias and classical Simon effect are not confounded. So, this task provides pure measures of these bias effects. This is, however, not true for a typical probeposition task, in which attentional bias and response bias are confounded (for further explanation, see Chapter 6, and Chapter 1, Section 1.2.2.3.).

Also in Chapter 7, a probe-classification task was applied in which all positions varied along the same dimension. In this chapter, the emphasis was on attentional bias and its temporal characteristics. No response bias effects were reported. Actually, no significant response bias effects were observed. It might be that the hypothesized response bias effects were overruled by the highly significant classical Simon effect that was evoked by the perceptually salient color information. In contrast to the probe classification task in Chapter 6, in Chapter 7, the color information eliciting a Simon effect appeared in half of the trials already at word onset.

At a structural level, the SAST is less complex than a probe-classification task. It includes only one contrast: the spatial correspondence between emotional stimulus and response (response bias). So, for the mere study of response bias, the simple SAST is preferred above the probe-classification task. 
Table 8.1. Structure of probe-classification task used in Chapter 6 (and 7).

\begin{tabular}{|c|c|c|c|c|c|}
\hline \multicolumn{6}{|c|}{$\begin{array}{c}\text { Probe-classification task } \\
\text { with positions varying along a single spatial dimension }\end{array}$} \\
\hline \multicolumn{3}{|c|}{ Positions } & \multicolumn{3}{|c|}{ Spatial correspondence } \\
\hline $\begin{array}{l}\text { Emotional } \\
\text { stimulus }\end{array}$ & Probe & Response & $\begin{array}{l}\text { Emotional stimulus } \\
\text { - probe }\end{array}$ & $\begin{array}{l}\text { Emotional stimulus } \\
\text { - response }\end{array}$ & Probe - response \\
\hline \multirow{4}{*}{ Top } & \multirow{2}{*}{ Top } & Top & Corresponding & Corresponding & Corresponding \\
\hline & & Bottom & Corresponding & Non-corresponding & Non-corresponding \\
\hline & \multirow{2}{*}{ Bottom } & Top & Non-corresponding & Corresponding & Non-corresponding \\
\hline & & Bottom & Non-corresponding & Non-corresponding & Corresponding \\
\hline \multirow{4}{*}{ Bottom } & \multirow{2}{*}{ Top } & Top & Non-corresponding & Non-corresponding & Corresponding \\
\hline & & Bottom & Non-corresponding & Corresponding & Non-corresponding \\
\hline & \multirow{2}{*}{ Bottom } & Top & Corresponding & Non-corresponding & Non-corresponding \\
\hline & & Bottom & Corresponding & Corresponding & Corresponding \\
\hline \multicolumn{3}{|c|}{ Non-corresponding minus corresponding = } & Attentional bias & Response bias & Classical Simon effect \\
\hline
\end{tabular}

\subsection{Preferential Processing in Anxiety: Future Research}

In the previous sections, the work conducted for this doctoral dissertation was critically reviewed. A number of issues on interpretation and methodology were put forward, and concrete suggestions for addressing them were offered. In this final section, we zoom out again and attend to some emerging themes and outstanding 
questions in today's research on preferential processing in anxiety, that were not directly addressed in the present experiments.

The present experiments, like most previous studies on preferential processing in anxiety, are correlational in design. They show associations between cognitive bias effects and anxiety, but they cannot shed light on the direction or causality of this relationship. To test the assumption that some biased cognitions play a causal role in anxiety and that changing these cognitions can reduce anxiety (e.g., Beck, 1976; Williams, Watts, MacLeod, \& Mathews, 1988, 1997; Mogg \& Bradley, 1998; Mathews \& Mackintosh, 1998), one needs an experimental design in which cognitive biases are systematically manipulated. There is some recent evidence suggesting that attentional and interpretive biases for emotional information can be induced by training and that such induced biases for negative information can modify anxiety vulnerability (e.g., MacLeod, Rutherford, Campbell, Ebsworthy, \& Holker, 2002; Mathews \& MacLeod, 2002; for reviews see MacLeod, Campbell, Rutherford, \& Wilson, 2004; Yiend \& Mackintosh, 2004). It has also been shown that re-training high-anxious individuals in directing attention away from threat or in interpreting ambiguous information in a non-threatening way results in reduced levels of anxiety (for reviews see MacLeod et al., 2004; Yiend \& Mackintosh, 2004). More research is needed to replicate and extend these first promising findings and to resolve more definitively which cognitive factors mediate and moderate anxiety. Retraining studies rely on the assumption that cognitive biases are, to a certain degree, controllable. More research is needed to get a better understanding of the degree to which these cognitive processes can be controlled and of the effects of effort, intention and mental resources (cf. Yiend, 2004b).

In addition to re-training specific cognitive biases for negative information, also training of attention in general may be helpful in reducing anxiety (e.g., Derryberry $\&$ Reed, 2002; Posner \& Rothbart, 2005). Individuals differ in how well they can voluntarily control their attention. Derryberry and Reed (2002) recently have shown that high-trait-anxious individuals, irrespective of how well they can control their attention, show an attentional bias for threat with short exposure durations. However, only those among them with poor attentional control still show this bias with longer exposure. It would be valuable for future research on preferential processing in anxiety to take individual differences in attentional control and other coping strategies into account. Derryberry and Reed (2002) used a standardized selfreport scale to assess differences in attentional control (shifting and focusing). Future research might also apply more objective measures of attention, general distractibility and interference (e.g., Attention Network Test: Fan, McCandliss, Sommer, Raz, \& Posner, 2002; endogenous cueing task combined with dot-probe task: Jongen, Smulders, Ranson, Arts, \& Krabbendam, in press; classical Simon task: Derryberry \& Reed, 2001 in Derryberry \& Reed, 2002). Attentional training has already been successfully applied in children with Attention Deficit / Hyperac- 
tivity Disorder, improving their attentional abilities (see Posner \& Rothbart, 2005). Posner and Rothbart have suggested that such attentional training might also be important for establishing a better regulation of emotions. It would be interesting to systematically test the effects of attentional training on anxiety levels and on bias effects associated with anxiety. Furthermore, it would be interesting to combine retraining of attentional bias effects with a general training in attentional control. The inclusion of the latter would be beneficial for those with poor control.

As stated before, there is a growing interest in the degree to which cognitive bias effects associated with anxiety can be controlled. Until recently, however, the focus was mainly on the automaticity of these bias effects, especially in attentional bias research (see Chapter 1, Section 1.2.3.; Yiend, 2004b). Several studies have been devoted to the role of stimulus awareness. Caution is, however, needed with the interpretation of most of these findings in terms of preconscious processing. It is hard to establish with sufficient certainty whether participants were completely unaware of every (type of) stimulus over the whole course of the task (cf. Mogg, Bradley, \& Williams, 1995). Future studies should address the role of awareness in a more precise way. Furthermore, it would be interesting to investigate other aspects of automaticity more thoroughly as well, such as the independence of the load imposed by a secondary task (e.g., cognitive or perceptual load).

The present experiments as well as the majority of studies on preferential processing in anxiety were confined to preferential processing of visual emotional information. It would be useful also to explore the other modalities.

To conclude, the aim of the present dissertation was to contribute to research on preferential processing in anxiety (1) by providing additional evidence for biased processing in terms of response bias and (2) by studying the temporal characteristics of anxiety-related attentional bias effects. In addition, this thesis provides a comprehensive review of behavioral research on preferential processing in anxiety.

\section{[...] This, the sum of experiments -}

\section{I loved them until they loved me. [...]}

Dorothy Parker (1926). Ballade at Thirty-Five (in Enough Rope) 



\section{Summary}

Over the past few decades, an increasing number of scientists have become convinced that anxiety has a substantial cognitive component: fundamental biases in information processing may play a crucial role in the etiology and maintenance of (disordered) anxiety. There is, for example, a growing body of evidence that anxiety is characterized by (automatic) preferential processing of threat-relevant information over other information. This evidence comes largely from behavioral studies during which emotional Stroop tasks, dot-probe tasks and visual search tasks have been applied. By using these reaction-time paradigms, researchers have made considerable progress in understanding anxiety-related preferential processing, its contentspecificity, time-course and automaticity.

The aim of this dissertation work was to contribute to a further understanding of preferential processing in anxiety. A novel approach was introduced to study early preferential processing of emotionally relevant information (Chapters 3-6). In addition, the temporal characteristics of anxiety-related selective attention were studied (Chapters 6-7). Chapter 1 provides an overview of the theoretical and empirical framework that has guided the research conducted for this dissertation.

\section{'Spatial Affective Simon’ Approach}

The key idea underlying the novel 'spatial affective Simon' approach is that if an emotional stimulus that is presented together with a neutral stimulus is preferentially processed, it may act as if it is presented alone. If so, it may yield a Simon effect: faster responses will be given when the position of the emotional stimulus corresponds with the response position than when these positions do not correspond. Such a Simon effect would add evidence to the hypothesis of early preferential processing of the emotional stimulus.

The Spatial Affective Simon Task (SAST) was designed as a measure of early preferential processing of emotionally relevant information. In the SAST, one emotional and one neutral stimulus appear simultaneously on a screen, and, crucially, the spatial correspondence of emotional stimulus and response varies. In the SAST as applied in this thesis, one emotional and one neutral word were presented one above the other, with the emotional word equally likely at the top or bottom position and with the word pair equally likely printed in red or green. Participants had to press the top key on a response box in response to a red word pair and the bottom key in response to a green word pair. As a result, in half of the trials the emotional word and the response key did spatially correspond (top-top, bottom- 
bottom), in the other half of the trials they did not correspond (top-bottom, bottom-top). Differences in response times between these corresponding and noncorresponding conditions reflect the existence of spatial affective Simon effects.

In Chapter 1, the rationale for the spatial affective Simon approach is given and the SAST is compared with the emotional Stroop task, dot-probe task, and nonspatial affective Simon paradigm.

Chapters 3-5 describe studies in which the SAST was applied to investigate early preferential processing of threat-relevant words in undergraduates exposed to a physical stressor (height; Chapter 3), in undergraduates with a high-anxious personality trait (Chapter 4), and in an anxious clinical sample (Chapter 5). In all three studies, word pairs were presented for 14 or 500 milliseconds (ms). Fourteen$\mathrm{ms}$ exposure was included to investigate whether anxiety-related preferential processing occurs under conditions of restricted awareness. With 500-ms exposure, there was more time for stimulus processing, enabling the operation of later control strategies.

Also in Chapter 6, spatial affective Simon effects were studied in high-traitanxious undergraduates, now using a probe-classification task. As in the SAST, one emotional and one neutral word were presented, one above the other, for 14 or 500 ms. Response position was again determined by color. However, here, the word pair was printed in white and followed by a red or green dot-probe, taking the position of one of the words. Consequently, bias effects were tapped later in time than in the SAST, in which the response-relevant property (color) was presented immediately at word onset. Furthermore, as color appears at only one of the locations (i.e. colored dot at top or bottom position), this probe-classification task can be used to study both spatial affective Simon effects ('response bias') and attentional bias effects. The latter reflected in the effects on response time of spatial correspondence of emotional stimulus and probe.

The main conclusions of the first four studies on spatial affective Simon effects can be summarized as follows: (1) Emotionally relevant words when presented together with a neutral word can yield a Simon effect, even under conditions of restricted stimulus awareness; (2) In all four studies, spatial affective Simon effects were observed for threat-relevant words in anxious persons and persons exposed to a stressor (faster responses when threat word and response spatially correspond). Nonanxious and low-anxious controls showed no significant effect or the opposite effect; (3) The observed spatial affective Simon effects were largely specific to current threat or concerns and to the situation; (4) The present evidence for anxiety-related spatial affective Simon effects is relevant for theories on biased processing associated with anxiety; (5) The SAST seems to be a promising tool to investigate preferential processing of emotional information, and may be useful in future research to acquire in-depth knowledge of the mechanisms associated with emotions and psychiatric disorders; (6) In the probe-classification study in Chapter 6, opposite response 
bias and attentional bias effects were observed, suggesting that these effects do not simply reflect one single mechanism. In typical probe-position tasks, the spatial position of the probe rather than the type of the probe determines the correct response; for example, press top key in response to probes on the top position, and bottom key in response to probes on the bottom position. Because of the direct spatial correspondence between emotional stimulus, probe and response in these tasks, response bias could contribute to the attentional bias scores. Therefore, probeposition tasks do not offer a pure measure of attentional bias.

\section{Temporal Dynamics of Selective Attention in Anxiety}

In recent years, researchers that were interested in the time-course of anxiety-related selective attention have manipulated exposure duration of the critical stimuli in dotprobe tasks, tracked eye movements or measured event-related brain activity. However, still little is known about the temporal characteristics of attentional bias in anxiety. In this dissertation, the temporal characteristics of attentional bias in anxiety were studied in reaction-time paradigms that were similar to a typical probeclassification task (see above) by analyzing distributions of response latencies (Chapter 6) and by independently manipulating stimulus duration and stimulus-probe onset asynchrony (SOA; Chapter 7). These were the first two studies in the attentional bias literature in which these analyses and manipulations were applied.

In Chapter 6 it is argued that relatively fast responses to the probe are more sensitive to early bias effects, whereas relatively slow responses are more influenced by later cognitive processes. Distributions of the response latencies obtained during the probe-classification experiment described before were analyzed. Faster responses when emotional stimulus and probe spatially correspond than when not were taken to reflect selective attention (attentional bias) to the emotional stimulus. In line with previous research, high-anxious compared to low-anxious undergraduates selectively attended to physical-threat words, when presented for $14 \mathrm{~ms}$ (limited to the first half of the task) and when presented for 500 ms. Interestingly, the group difference observed with 14-ms exposure increased with slower responses; with 500-ms exposure, the group difference did not depend on response speed. These results were explained in terms of delayed disengagement of attention from threat-related content in anxiety. It was concluded that distributional analyses of response latencies on a dot-probe task may help to reveal the time-course of attentional bias.

Chapter 7 examines the time-course of anxiety-related attentional bias effects and the influence of processing time on these effects. High-trait-anxious and lowtrait-anxious undergraduates performed a classification task during which one emotional and one neutral word were presented, one above the other, for 14 or 500 ms. Following this masked word pair, a dot appeared at each location previously 
occupied by a word. Color information was added to the display to determine the response (top response to red, bottom response to green). Word-color SOA was 0 $\mathrm{ms}$ (one of the words colored) or $514 \mathrm{~ms}$ (words in white, one of the dots colored). The color appeared equally likely at the upper or lower position, and thus took the role of the probe in conventional dot-probe tasks. By independently manipulating word duration and SOA, it was possible to experimentally dissociate the effects of processing time and moment of tapping, respectively. Importantly, bias effects elicited by physical-threat, social-threat and positive words differed in temporal characteristics, suggesting specificity of anxiety-related bias. As a methodological note, there were differences in bias scores between threat words presented for 14 and $500 \mathrm{~ms}$, given 0 -ms SOA, suggesting that these scores may reflect attentional allocation after probe onset.

\section{General Discussion}

Chapter 8 reviews the empirical findings in the preceding chapters and addresses several issues on interpretation and methodology. Alternative explanations for the observed spatial affective Simon effects are discussed: extreme enhanced processing of the emotional stimulus, response tendencies to approach or avoid the emotional stimulus, and attentional orienting. Furthermore, the observed differences between task blocks, the stimulus material used and the specific design of the tasks applied are discussed. Finally, some topical questions in research on preferential processing in anxiety that were not directly addressed by the experiments in this dissertation are described, such as causal factors, (re-)training of attentional bias, and attentional control. 


\section{Samenvatting}

In de loop van de laatste decennia zijn steeds meer wetenschappers ervan overtuigd geraakt dat angst een belangrijke cognitieve component omvat. Ze menen dat structurele afwijkingen in de verwerking van informatie een cruciale rol spelen bij het ontstaan en blijven bestaan van (abnormale) angst. Er is bijvoorbeeld een toenemende evidentie dat angst gekenmerkt wordt door een (automatische) neiging om bij voorkeur die informatie te verwerken die gerelateerd is aan de bron van angst. Deze evidentie werd grotendeels verkregen door middel van gedragsstudies waarin emotionele Stroop taken, dot-probe taken en visuele zoektaken werden gebruikt. Door het toepassen van deze reactietijdparadigma's hebben onderzoekers aanzienlijke vooruitgang geboekt in het begrijpen van de preferentiële informatieverwerking bij angst, haar specificiteit, tijdsverloop en automaticiteit.

Het doel van het onderzoek beschreven in dit proefschrift was om meer inzicht te verkrijgen in de preferentiële informatieverwerking bij angst. Een nieuwe benadering werd geïntroduceerd om de vroege voorkeursverwerking van emotioneel relevante informatie te bestuderen (Hoofdstukken 3-6). Daarnaast werden de temporele eigenschappen van selectieve aandacht bij angst onder de loep genomen (Hoofdstukken 6-7). Hoofdstuk 1 biedt een overzicht van het theoretisch en empirisch kader dat dit onderzoek heeft gestuurd.

\section{'Spatiële affectieve Simon' benadering}

Het centrale idee achter de nieuwe 'spatiële affectieve Simon' benadering is als volgt: als een emotionele stimulus, die samen met een neutrale stimulus wordt gepresenteerd, bij voorkeur wordt verwerkt, dan gaat hij zich misschien gedragen alsof hij alleen werd gepresenteerd. Als dat zo is, dan kan hij een Simon effect voortbrengen: er zal sneller gereageerd worden wanneer de ruimtelijke positie van de emotionele stimulus correspondeert met de positie van de respons dan wanneer deze posities niet met elkaar corresponderen. Zo een Simon effect zou extra evidentie bieden voor het idee van vroege preferentiële verwerking van de emotionele stimulus.

De Spatiële Affectieve Simon Taak (SAST) werd ontwikkeld als een maat voor de vroege voorkeursverwerking van emotionele informatie. In de SAST verschijnen tegelijkertijd een emotionele en een neutrale stimulus op een computerscherm en varieert de correspondentie tussen de positie van de emotionele stimulus en de positie van de respons. In de SAST zoals toegepast in dit proefschrift worden een emotioneel en een neutraal woord boven elkaar aangeboden. Het emotionele woord komt met een even grote waarschijnlijkheid op de bovenste als op de onderste 
positie en het woordpaar is even vaak in het rood als in het groen gedrukt. Aan de proefpersonen werd gevraagd op de bovenste knop van een responskastje te drukken wanneer een rood woordpaar zou verschijnen, op de onderste knop wanneer een groen paar zou verschijnen. Het resultaat was dat tijdens de helft van de keren dat een woordpaar werd aangeboden de posities van het emotionele woord en de responsknop met elkaar correspondeerden (boven-boven, onder-onder) en dat tijdens de andere helft deze posities niet correspondeerden (boven-onder, onder-boven). Het verschil in reactietijd tussen deze spatieel corresponderende en nietcorresponderende condities weerspiegelt het bestaan van spatiële affectieve Simon effecten.

In Hoofdstuk 1 wordt het basisidee voor de spatiële affectieve Simon benadering beschreven en wordt de SAST vergeleken met de emotionele Stroop taak, de dot-probe taak, en een niet-spatieel affectief Simon paradigma. Hoofdstukken 3-5 rapporteren studies waarin de SAST werd toegepast om de vroege voorkeursverwerking van dreigwoorden te onderzoeken bij studenten die werden blootgesteld aan een gebeurtenis die fysieke stress kan veroorzaken (hoogte; Hoofdstuk 3), bij studenten met een hoogangstige persoonlijkheid (Hoofdstuk 4) en bij een angstige klinische groep (Hoofdstuk 5). In alle drie de studies werden woordparen gepresenteerd gedurende 14 of 500 milliseconden (ms). Er werd gekozen voor een aanbiedingsduur van $14 \mathrm{~ms}$ om zo te kunnen onderzoeken of de veronderstelde preferentiele informatieverwerking bij angst plaatsvindt wanneer de stimuli niet volledig bewust worden waargenomen. Bij een aanbiedingsduur van $500 \mathrm{~ms}$ is er meer tijd om de stimulus te verwerken, waardoor latere strategische processen in werking kunnen treden.

Ook in Hoofdstuk 6 worden spatiële affectieve Simon effecten bestudeerd bij studenten met een hoogangstige persoonlijkheid, maar nu door middel van een probe-classificatie taak. Net zoals in de SAST werden een emotioneel en een neutraal woord boven elkaar gepresenteerd gedurende 14 of $500 \mathrm{~ms}$ en ook hier werd responspositie bepaald door de kleur. In de probe-classificatie taak werd het woordpaar echter in het wit gedrukt en gevolgd door een rode of groene stip, de 'probe', die de positie van een van de woorden innam. Zodoende werden de effecten op een later moment in de tijd gemeten dan in de SAST, waar de responsrelevante eigenschap (kleur) tegelijk met de woorden verscheen. Bovendien, omdat de kleur maar op een van de locaties verschijnt (bijv. gekleurde stip op de bovenste of op de onderste positie) kan deze probe-classificatie taak gebruikt worden om zowel spatiële affectieve Simon effecten ('responsbias') te onderzoeken als selectieve aandachtseffecten ('aandachtsbias'). Aandachtsbias wordt gereflecteerd in de effecten van de spatiële correspondentie tussen de emotionele stimulus en de stip op reactiesnelheid.

De belangrijkste conclusies van de eerste vier studies naar spatiële affectieve Simon effecten kunnen als volgt worden samengevat: (1) Emotionele woorden die samen met een neutraal woord worden gepresenteerd, kunnen een Simon effect 
uitlokken, zelfs wanneer de woorden niet volledig bewust worden waargenomen; (2) In alle vier de studies werden spatiële affectieve Simon effecten gevonden voor dreigwoorden bij angstige personen en bij personen die werden blootgesteld aan een stressveroorzakende gebeurtenis (reacties waren sneller wanneer dreigwoord en respons spatieel correspondeerden). Niet-angstige en laagangstige controlegroepen toonden geen effect ofwel het tegenovergestelde effect; (3) De geobserveerde spatiële affectieve Simon effecten waren in grote mate specifiek voor de bedreiging of voor iemand's zorgen en voor de situatie op het moment van meten; (4) Deze angstgerelateerde spatiële affectieve Simon effecten zijn relevant voor theorieën over afwijkende informatieverwerking bij angst; (5) De SAST is een veelbelovend paradigma om voorkeursverwerking van emotionele informatie te onderzoeken en kan misschien nuttig zijn in toekomstig onderzoek om diepgaande kennis te verwerven over de mechanismen die een rol spelen bij emoties en psychiatrische stoornissen; (6) In de probe-classificatie studie in Hoofdstuk 6 werden tegenovergestelde responsbias en aandachtsbias effecten gevonden. Dit suggereert dat deze effecten niet eenvoudigweg een reflectie zijn van een en hetzelfde onderliggende mechanisme. In typische probepositie taken bepaalt de positie van de probe, en niet het type probe, de correcte respons; bijvoorbeeld, druk op de bovenste knop wanneer de probe op de bovenste positie verschijnt en op de onderste knop wanneer zij op de onderste positie verschijnt. Omdat er in deze taken een directe correspondentie bestaat tussen de posities van emotionele stimulus, probe en respons, zou responsbias kunnen bijdragen aan de aandachtsbias-scores. Probe-positie taken vormen dus geen zuivere meting van aandachtsbias.

\section{Temporele dynamiek van selectieve aandacht in angst}

De afgelopen jaren hebben onderzoekers die geïnteresseerd waren in het tijdsverloop van selectieve aandacht bij angst, de aanbiedingsduur van de kritieke stimuli in dotprobe taken gemanipuleerd en oogbewegingen en hersenactiviteit gemeten. Op dit moment is er echter nog steeds weinig geweten over de temporele eigenschappen van aandachtsbias bij angst. In dit proefschrift werden deze eigenschappen bestudeerd in reactietijdparadigma's die lijken op een typische probe-classificatie taak (hierboven beschreven), door het analyseren van reactietijdverdelingen (Hoofdstuk 6) en door het apart manipuleren van de stimulusduur en van het interval tussen het verschijnen van de emotionele stimulus en het verschijnen van de probe (stimulus onset asynchrony; SOA; Hoofdstuk 7). Dit waren de eerste twee studies in de literatuur over aandachtsbias waarin deze analyses en manipulaties werden toegepast.

In Hoofdstuk 6 wordt beargumenteerd dat relatief snelle responsen op de probe gevoeliger zijn voor vroege bias effecten, terwijl relatief langzame responsen meer beïnvloed worden door latere cognitieve processen. De distributies van reactietijden 
gemeten tijdens het hierboven beschreven probe-classificatie experiment werden geanalyseerd. Snellere responsen wanneer de emotionele stimulus en de probe spatieel correspondeerden dan wanneer ze dat niet deden, werden gezien als een reflectie van aandachtsbias voor de emotionele stimulus. In overeenstemming met eerder onderzoek gaven hoogangstige studenten, in vergelijking met laagangstige studenten, selectief aandacht aan woorden die gerelateerd waren aan fysieke bedreiging, bij een aanbiedingsduur van $14 \mathrm{~ms}$ (enkel tijdens de eerste taakhelft) of 500 ms. Het groepsverschil bij een aanbiedingsduur van $14 \mathrm{~ms}$ werd groter bij langzamere responsen; bij een aanbiedingsduur van 500 ms hing het groepsverschil niet af van reactiesnelheid. Deze resultaten werden verklaard in termen van het moeilijk kunnen losmaken van aandacht van stimuli die gerelateerd zijn aan dreiging bij angst. Er werd geconcludeerd dat analyses van verdelingen van reactietijden op een dot-probe taak van dienst kunnen zijn bij het in kaart brengen van het tijdsverloop van aandachtsbias.

Hoofdstuk 7 onderzoekt het tijdsverloop van andachtsbias bij angst en de invloed van de duur van stimulusverwerking hierop. Hoogangstige en laagangstige studenten voerden een classificatietaak uit waarin een emotioneel en een neutraal woord boven elkaar werden gepresenteerd, gedurende 14 of 500 ms. Onmiddellijk na dit gemaskeerde woordpaar verscheen er een stip op elke positie waar eerder een woord stond. Kleurinformatie werd an het beeldscherm toegevoegd om de respons te bepalen (bovenste knop wanneer rood, onderste knop wanneer groen). Woordkleur SOA was $0 \mathrm{~ms}$ (een van de woorden in kleur) of $514 \mathrm{~ms}$ (woorden in het wit, een van de stippen in kleur). De kleur verscheen even waarschijnlijk op de bovenste als op de onderste positie en nam dus de rol over van de probe in een conventionele dot-probe taak. Door aanbiedingsduur en SOA onafhankelijk van elkaar te manipuleren, was het mogelijk om de effecten van, respectievelijk, verwerkingsduur en het moment van meten experimenteel te scheiden.

Een interessante bevinding van deze studie was dat de bias effecten die werden uitgelokt door woorden gerelateerd aan fysieke dreiging, woorden gerelateerd aan sociale dreiging en positieve woorden van elkaar verschilden in temporele eigenschappen. Dit suggereert specificiteit van bias bij angst. Bij wijze van methodologische noot is het relevant te melden dat er bij 0 -ms SOA een verschil was tussen bias scores voor bedreigende woorden gepresenteerd voor $14 \mathrm{~ms}$ en scores voor deze woorden gepresenteerd voor 500 ms. Dit suggereert dat deze bias scores reflecteren waar aandacht zit na het verschijnen van de probe.

\section{Algemene discussie}

Hoofdstuk 8 geeft een overzicht van de empirische bevindingen in de voorgaande hoofdstukken en bespreekt verschillende kwesties met betrekking tot interpretatie en 
methodologie. Alternatieve verklaringen voor de geobserveerde spatiële affectieve Simon effecten worden bediscussieerd: een extreme versterkte verwerking van de emotionele stimulus, responsneigingen om de emotionele stimulus te benaderen of te vermijden, het richten van aandacht. Verder worden ook de geobserveerde verschillen tussen taakblokken, het gebruikte stimulusmateriaal en het specifieke design van de gebruikte taken bediscussieerd. Tot slot worden enkele vragen besproken die op dit moment erg actueel zijn in het onderzoek naar preferentiële informatieverwerking bij angst, maar die niet direct werden aangepakt in de experimenten in dit proefschrift, bijvoorbeeld causale factoren, (re-)training van aandachtsbias, aandachtscontrole. 



\section{References}

A

Algom, D., Chajut, E., \& Lev, S. (2004). A rational look at the Emotional Stroop Phenomenon: A generic slowdown, not a Stroop effect. Journal of Experimental Psychology: General, 133, 323-338.

Allport, D. A. (1987). Selection for action: Some behavioural and neurophysiological considerations of attention and action. In H. Heuer \& A. F. Sanders (Eds.), Perspectives on perception and action (pp. 395419). Hillsdale, NJ: Lawrence Erlbaum Associates Inc.

Amir, N., Elias, J., Klumpp, H., \& Przeworski, A. (2003). Attentional bias to threat in social phobia: Facilitated processing of threat or difficulty disengaging attention from threat? Behaviour Research and Therapy, 41, 1325-1335.

Amir, N., McNally, R. J., Riemann, B. C., Burns, J., Lorenz, M., \& Mullen, J. T. (1996). Suppression of the emotional Stroop effect by increased anxiety in patients with social phobia. Behaviour Research and Therapy, 34, 945-948.

Armony, J. L., \& Dolan, R. J. (2002). Modulation of spatial attention by fear-conditioned stimuli: An eventelated fMRI study. Neuropsychologia, 40, 817-826.

Armony, J. L., \& LeDoux,, J. E. (2000). How danger is encoded: Towards a systems, cellular, and computaional understanding of cognitive-emotional interactions. In M. S. Gazzaniga (Ed.), The new cognitive neurosciences $\left(2^{\text {nd }}\right.$ ed., pp. 1067-1079). Cambridge, MA: MIT Press.

Asmundson, G. J. G., \& Stein, M. B. (1994). Selective processing of social threat in patients with generalized social phobia: Evaluation using a dot-probe paradigm. Journal of Anxiety Disorders, 8, 107-117.

B

Baaijen, R. H., Piepenbrock, R, \& Gulikers, L., The CELEX Lexical Database (CD-ROM). Linguistic Data Consortium, University of Pennsylvania, Philadelphia, PA, 1995.

Baas, J. M. P., Kenemans, J. L., Bőcker, K. B. E., \& Verbaten, M. N. (2002). Threat-induced cortical processing and startle potentiation. Neuroreport, 13, 133-137.

Bargh, J. A. (1992). The ecology of automaticity: Toward establishing the conditions needed to produce automatic processing effects. American Journal of Psychology, 105, 181-199.

Bar-Haim, Y., Lamy, D., Pergamin, L., Bakermans-Kranenburg, M. J., \& van IJzendoorn, M. H. (2007). Threat-related attentional bias in anxious and nonanxious individuals: A meta-analytic study. Psychological Bulletin, 133, 1-24.

Beck, A. T. (1976). Cognitive therapy and the emotional disorders. New York: International Universities Press.

Beck, A. T., Ward, C. H., Mendelson, M., Mock, J., \& Erbaugh, J. (1961). An inventory for measuring depression. Archives of General Psychiatry, 4, 561-571.

Beringer, J. (1987). Experimental Run Time System (Version 3.32c). Frankfurt, Germany: Berisoft Cooperation.

Blanchard, R. J., \& Blanchard, D. C. (1990). An ethoexperimental analysis of defense, fear and anxiety. In N. McNaughton \& G. Andrews (Eds.), Anxiety (pp. 124-133). Dunedin: Otago University Press.

Bower, G. H. (1981). Mood and memory. American Psychologist, 36, 129-148.

Bradley, B. P., Mogg, K., Falla, S. J., \& Hamilton, L. R. (1998). Attentional bias for threatening facial expressions in anxiety: Manipulation of stimulus duration. Cognition and Emotion, 12, 737-753.

Bradley, B. P., Mogg, K., \& Lee, S. C. (1997). Attentional biases for negative information in induced and naturally occurring dysphoria. Behaviour Research and Therapy, 35, 911-927.

Bradley, B. P., Mogg, K., \& Millar, N. (2000). Biases in overt and covert orienting to emotional facial expressions. Cognition and Emotion, 14, 789-808.

Bradley, B. P., Mogg, K., Millar, N., \& White, J. (1995). Selective processing of negative information: Effects of clinical anxiety, concurrent depression, and awareness. Journal of Abnormal Psychology, 104, 532-536.

Bradley, B. P., Mogg, K., White, J., Groom, C., \& de Bono, J. (1999). Attentional bias for emotional faces in generalized anxiety disorder. British Journal of Clinical Psychology, 38, 267-278. 


\section{References}

Broadbent, D., \& Broadbent, M. (1988). Anxiety and attentional bias: State and trait. Cognition and Emotion, 2, $165-183$.

Broomfield, N. M., \& Turpin, G. (2005). Covert and overt attention in trait anxiety: a cognitive psychophysiological analysis. Biological Psychology, 68, 179-200.

Brosschot, J. F., de Ruiter, C., \& Kindt, M. (1999). Processing bias in anxious subjects and repressors, measured by emotional Stroop interference and attentional allocation. Personality and Individual Differences, 26, 777-793.

Byrne, A., \& Eysenck, M. W. (1995). Trait anxiety, anxious mood, and threat detection. Cognition and Emotion, 9, 549-562.

C

Cacioppo, J. T., Priester, J. R., \& Berntson, G. G. (1993). Rudimentary determinants of attitudes: II. Arm flexion and extension have differential effects on attitudes. Journal of Personality and Social Psychology, 65, 5-17.

Chajut, E., Lev, S., \& Algom, D. (2005). Vicissitudes of a Misnomer: Reply to Dalgleish (2005). Journal of Experimental Psychology: General, 134, 592-595.

Cheesman, J., \& Merikle, P.M. (1986). Distinguishing conscious from unconscious perceptual processes. Canadian Journal of Psychology, 40, 343-367.

Chen, M., \& Bargh, J. A. (1999). Nonconscious approach and avoidance behavioral consequences of the automatic evaluation effect. Personality and Social Psychology Bulletin, 25, 215-224.

Chen, Y. P., Ehlers, A., Clark, D. M., Mansell, W. (2002). Patients with social phobia direct their attention away from faces. Behaviour Research and Therapy, 40, 677-687.

Christie, J., \& Klein, R. (1995). Familiarity and attention: Does what we know affect what we notice? Memory and Cognition, 23, 547-550.

Cohen, J. D., Dunbar, K., \& McClelland, J. L. (1990). On the control of automatic processes: A parallel distributed processing account of the Stroop effect. Psychological Review, 97, 332-361.

Cohen, J. D., Servan Schreiber, D., \& McClelland, J. L. (1992). A parallel distributed processing approach to automaticity. American Journal of Psychology, 105, 239-269.

Collie, A., Maruff, P., Yucel, M., Danckert, J., \& Currie, J. (2000). Spatiotemporal distribution of facilitation and inhibition of return arising from the reflexive orienting of covert attention. Journal of Experimental Psychology: Human Perception and Performance, 26, 1733-1745.

Compton, R. J. (2003). The interface between emotion and attention: A review of evidence from psychology and neuroscience. Behavioral and Cognitive Neuroscience Reviews, 2, 115-129.

Cooper, R. M., \& Langton, S. R. H. (2006). Attentional bias to angry faces using the dot-probe task? It depends when you look for it. Behaviour Research and Therapy, 44, 1321-1329.

Craft, J. L., \& Simon, J. R. (1970). Processing symbolic information from a visual display: Interference from an irrelevant directional cue. Journal of Experimental Psychology, 83, 415-420.

$\mathrm{D}$

Dalgleish, T. (2005). Putting Some Feeling Into It -The Conceptual and Empirical Relationships Between the Classic and Emotional Stroop Tasks: Comment on Algom, Chajut, and Lev (2004). Journal of Experimental Psychology: General, 134, 585-591.

Davis, M. (1992a). The role of the amygdala in conditioned fear. In J. P. Aggleton (Ed.), The amygdala: Neurobiological aspects of emotion, memory, and mental dysfunction (pp. 255-306). New York, NY: WileyLiss.

Davis, M. (1992b). The role of the amygdala in fear and anxiety. Annual Review of Neuroscience, 15, 353-375.

Davis, M., \& Whalen, P. J. (2001). The amygdala: Vigilance and emotion. Molecular Psychiatry, 6, 13-34.

De Houwer, J. (2003). On the role of stimulus-response and stimulus-stimulus compatibility in the Stroop effect. Memory and Cognition, 31, 353-359.

De Houwer, J., Crombez, G., Baeyens, F., \& Hermans, D. (2001). On the generality of the affective Simon effect. Cognition and Emotion, 15, 189-206.

De Houwer, J., \& Eelen, P. (1998). An affective variant of the Simon paradigm. Cognition and Emotion, 12, 45-61.

De Jong, R., Liang, C.-C., \& Lauber, E. (1994). Conditional and unconditional automaticity: A dual-process 
model of effects of spatial stimulus-response compatibility. Journal of Experimental Psychology: Human Perception and Performance, 20, 731-750.

De Jong, R., Wierda, M., Mulder, G., \& Mulder, L. J. M. (1988). Use of partial stimulus information in response processing. Journal of Experimental Psychology: Human Perception and Performance, 14, 682-692.

Derryberry, D. (1991). The immediate effects of positive and negative feedback signals. Journal of Personality and Social Psychology, 61, 267-278.

Derryberry, D., \& Reed, M. (2002). Anxiety-related attentional biases and their regulation by attentional control. Journal of Abnormal Psychology, 111, 225-236.

De Ruiter, C., \& Brosschot, J. F. (1994). The emotional Stroop interference effect in anxiety: Attentional bias or cognitive avoidance? Behaviour Research and Therapy, 32, 315-319.

Dijksterhuis, A., \& Aarts, H. (2003). On wildebeests and humans: The preferential detection of negative stimuli. Psychological Science, 14, 14-18.

Dixon, N. F. (1971). Subliminal perception: The nature of controversy. New York: McGraw-Hill.

E

Eastwood, J. D., Smilek, D., \& Merikle, P. M. (2001). Differential attentional guidance by unattended faces expressing positive and negative emotion. Perception and Psychophysics, 63, 1004-1013.

Egloff, B., \& Hock, M. (2003). Assessing attention allocation toward threat-related stimuli: A comparison of the emotional Stroop task and the attentional probe task. Personality and Individual Differences, 35, 475483.

Eimer, M. (1996). The N2pc component as an indicator of attentional selectivity. Electroencephalography and Clinical Neurophysiology, 99, 225-234.

Eimer, M., \& Kiss, M. (2007). Attentional capture by task-irrelevant fearful faces is revealed by the N2pc component. Biological Psychology, 74, 108-112.

Ekman, P., \& Friesen, W. (1976). Emotion in the human face. New York: Cambridge University Press.

Endler, N. S (1983). Interactionism: A personality model, but not yet a theory. In M. M. Page (Ed.), Nebraska Symposium on Motivation: Personality - Current Theory and Research. Lincoln: University of Nebraska Press.

Esteves, F., Dimberg, U., \& Öhman, A. (1994). Automatically elicited fear: Conditioned skin conductance responses to masked facial expressions. Cognition and Emotion, 8, 393-413.

Eysenck, M. W. (1992). Anxiety: The cognitive perspective. Hillsdale, NJ: Lawrence Erlbaum Associates.

Eysenck, M. W. (1997). Anxiety and cognition: A unified theory. Hove: Psychology Press.

Eysenck, M. W. (2000). A cognitive approach to trait anxiety. European Journal of Personality, 14, 463-476.

Eysenck, M. W., \& Byrne, A. (1992). Anxiety and susceptibility to distraction. Personality and Individual Differences, 13, 793-798.

F

Fan, J., McCandliss, B. D., Sommer, T., Raz, A., \& Posner, M. I. (2002). Testing the efficiency and independence of attentional networks. Journal of Cognitive Neuroscience, 14, 340-347.

Faust, M. E., Balota, D. A., Spieler, D. H., \& Ferraro, F. R. (1999). Individual differences in informationprocessing rate and amount: Implications for group differences in response latency. Psychological Bulletin, 125, 777-799.

First, M. B., Spitzer, R. L., Gibbon, M., \& Williams, J. B. W. (1997). Structured clinical interview for DSM-IV axis I disorders (SCID-I). New York: New York State Psychiatric Institute.

First, M. B., Spitzer, R. L., Gibbon, M., Williams, J. B. W, \& Benjamin, L. (1994). Structured clinical interview for DSM-IV axis II personality disorders (SCID-II). New York: New York State Psychiatric Institute.

Fox, E. (1993a). Allocation of visual attention and anxiety. Cognition and Emotion, 7, 207-215.

Fox, E. (1993b). Attentional bias in anxiety: Selective or not? Behavioural Research and Therapy, 31, 487493.

Fox, E. (1994). Attentional bias in anxiety: A defective inhibition hypothesis. Cognition and Emotion, 8, 165195.

Fox., E. (1996). Selective processing of threatening words in anxiety: The role of awareness. Cognition and Emotion, 10, 449-480.

Fox, E. (2002). Processing emotional facial expressions: The role of anxiety and awareness. Cognitive, Affective 


\section{References}

and Behavioral Neuroscience, 2, 52-63.

Fox, E. (2004). Maintenance or capture of attention in anxiety-related biases? In J. Yiend (Ed.), Cognition, emotion and psychopathology: Theoretical, empirical and clinical directions (pp. 86-105). Cambridge, UK: Cambridge University Press.

Fox, E., Lester, V., Russo, R., Bowles, R., Pichler, A., \& Dutton, K. (2000). Facial expressions of emotion: Are angry faces detected more efficiently? Cognition and Emotion, 14, 61-92.

Fox, E., Russo, R., Bowles, R., \& Dutton, K. (2001). Do threatening stimuli draw or hold visual attention in subclinical anxiety? Journal of Experimental Psychology: General, 130, 681-700.

Fox, E., Russo, R., \& Dutton, K. (2002). Attentional bias for threat: Evidence for delayed disengagement from emotional faces. Cognition and Emotion, 16, 355-379.

Fox, E., Russo, R., \& Georgiou, G. A. (2005). Anxiety modulates the degree of attentive resources required to process emotional faces. Cognitive, Affective, \& Behavioral Neuroscience, 5, 396-404.

Frijda, N. H. (1986). The emotions. Cambridge, UK: Cambridge University press.

G

Garner, M., Mogg, K., \& Bradley, B. P. (2006). Orienting and maintenance of gaze to facial expressions in social anxiety. Journal of Abnormal Psychology, 115, 760-770.

Georgiou, G. A., Bleakley, C., Hayward, J., Russo, R., Dutton, K., Eltiti, S., \& Fox., E. (2005). Focusing on fear: Attentional disengagement from emotional faces. Visual Cognition, 12, 145-158.

Gibson, B. S., \& Bryant, T. A. (2005). Variation in cue duration reveals top-down modulation of involuntary orienting to uninformative symbolic cues. Perception and Psychophysics, 67, 749-758.

Gilboa-Schechtman, E., Foa, E. B., \& Amir, N. (1999). Attentional biases for facial expressions in social phobia: The face-in-the-crowd paradigm. Cognition and Emotion, 13, 305-318.

Gotlib, I. H., McLachlan, A. L., \& Katz, A. N. (1988). Biases in visual attention in depressed and nondepressed individuals. Cognition and Emotion, 2, 185-200.

Gratton, G., Coles, M. G. H., Sirevaag, E. J., Eriksen, C. W., \& Donchin, E. (1988). Pre- and poststimulus activation of response channels: A psychophysiological analysis. Journal of Experimental Psychology: Human Perception and Performance, 14, 331-344.

Gray, J. A., \& McNaughton, N. (2003). The neuropsychology of anxiety (2 ${ }^{\text {nd }}$ Ed.). NY: Oxford University Press Inc.

Groenestijn, M. A. C., Akkerhuis, G. W., Kupka, R. W., Schneider, N., \& Nolen, W. A. (1999). Gestructureerd klinisch interview voor de vaststelling van DSM-IV as-I stoornissen (SCID-I) [Structured clinical interview for DSM-IV axis I disorders (SCID-I)]. Lisse, Netherlands: Swets \& Zeitlinger.

$\mathrm{H}$

Hajcak, G., Molnar, C., George, M. S., Bolger, K., Koola, J., \& Nahas, Z. (2007). Emotion facilitates action: A transcranial magnetic stimulation study of motor cortex excitability during picture viewing. Psychophysiology, 44, 91-97.

Hampton, C., Purcell, D. G., Bersine, L., Hansen, C. H., \& et al. (1989). Probing "pop-out": Another look at the face-in-the-crowd effect. Bulletin of the Psychonomic Society, 27, 563-566.

Hansen, C. H., \& Hansen, R. D. (1988). Finding the face in the crowd: An anger superiority effect. Journal of Personality and Social Psychology, 54, 917-924.

Harmon-Jones, E. (2003). Anger and the behavioral approach system. Personality and Individual Differences, 35, $995-1005$.

Hasbroucq, T., \& Guiard, Y. (1991). Stimulus response compatibility and the Simon effect: Toward a conceptual clarification. Journal of Experimental Psychology: Human Perception and Performance, 17, 246266.

Hedge, A., \& Marsh, N. W. (1975). The effect of irrelevant spatial correspondence on two-choice responsetime. Acta Psychologica, 39, 427-439.

Hermans, H. (1967). Een vragenlijst voor het prestatie-motief en de invloed van sociale wenselijkheid [A questionnaire measure of achievement motivation and the influence of social-desirability]. In H. Hermans (Ed.), Motivatie en prestatie (pp.62-79). Amsterdam, Netherlands: Swets.

Hermans, D., De Houwer, J., \& Eelen, P. (2001). A time course analysis of the affective priming effect. Cognition and emotion, 15, 143-165.

Hermans, D., Vansteenwegen, D., \& Eelen, P. (1999). Eye movement registration as a continuous index of 
attention deployment: Data from a group of spider anxious students. Cognition and Emotion, 13, 419434.

Holender, D. (1986). Semantic activation without conscious identification in dichotic listening, parafoveal vision, and visual masking: A survey and appraisal. The Behavioral and Brain Sciences, 9, 1-66.

Hommel, B. (1993a). The role of attention for the Simon effect. Psychological Research, 55, 208-222.

Hommel, B. (1993b). The relationship between stimulus processing and response selection in the Simon task: Evidence for a temporal overlap. Psychological Research, 55, 280-290.

Hope, D. A., Rapee, R. M., Heimberg, R. G., \& Dombeck, M. J. (1990). Representations of the self in social phobia: Vulnerability to social threat. Cognitive Therapy and Research, 14, 177-189.

Howell, D. C. (2002). Statistical methods for psychology (5 $5^{\text {th }}$ Ed.). Pacific Grove: Duxbury.

I

Ioannou, M. C., Mogg, K., \& Bradley, B. P. (2004). Vigilance for threat: effects of anxiety and defensiveness. Personality and Individual Differences, 36, 1879-1891.

Ivanoff, J., \& Peters, M. (2000). A shift of atttention may be necessary, but it is not sufficient, for the generation of the Simon effect. Psychological Research, 64, 117-135.

J

Johnson Laird, P. N., Mancini, F., \& Gangemi, A. (2006). A hyper-emotion theory of psychological illnesses. Psychological Review, 113, 822-841.

Jones, G. V., Stacey, H., \& Martin, M. (2002). Exploring the intensity paradox in emotional Stroop interference. Cognitive Therapy and Research, 26, 831-839.

Jongen, E. M. M., Smulders, F. T. Y., Ranson, S. M. G., Arts, B. M. G., \& Krabbendam, L. (in press). Attentional bias and general orienting processes in bipolar disorder. Journal of Behavior Therapy and Experimental Psychiatry.

Jonides, J. (1981). Voluntary versus automatic control over the mind's eye's movement. In J. B. Long \& A. D. Baddeley (Eds.), Attention and Performance IX (pp. 187-203). Hillsdale, NJ: Lawrence Erlbaum.

K

Kleinbaum, D. G., Kupper, L. L., Muller, K. E., \& Nizam, A. (1998). Applied regression analysis and multivariable methods ( $3^{\text {rd }}$ Ed.). Pacific Grove: Duxbury.

Kornblum, S., Hasbroucq, T., \& Osman, A. (1990). Dimensional overlap: Cognitive basis for stimulusresponse compatibility - A model and taxonomy. Psychological Review, 97, 253-270.

Koster, E. H. W., Crombez, G., Van Damme, S., Verschuere, B., \& De Houwer, J. (2004). Does imminent threat capture and hold attention? Emotion, 4, 312-317.

Koster, E. H. W., Crombez, G., Verschuere, B., \& De Houwer, J. (2004). Selective attention to threat in the dot probe paradigm: Differentiating vigilance and difficulty to disengage. Behaviour Research and Therapy, 42, 1183-1192.

Koster, E. H. W., Crombez, G., Verschuere, B., Van Damme, S., \& Wiersema, J. R. (2006). Components of attentional bias to threat in high trait anxiety: Facilitated engagement, impaired disengagement, and attentional avoidance. Behaviour Research and Therapy, 44, 1757-1771.

Koster, E. H. W., Crombez, G., Van Damme, S., Verschuere, B., \& De Houwer, J. (2005). Signals for threat modulate attentional capture and holding: Fear-conditioning and extinction during the exogenous cueing task. Cognition and Emotion, 19, 771-780.

Koster, E. H. W., Verschuere, B., Crombez, G., \& Van Damme, S. (2005). Time-course of attention for threatening pictures in high and low trait anxiety. Behaviour Research and Therapy, 43, 1087-1098.

$\mathrm{L}$

LaBerge, D. (1995). Networks of attention. In M. S. Gazzaniga (Ed.), The new cognitive neurosciences ( $2^{\text {nd }}$ ed., pp. 711-724). Cambridge, MA: MIT Press.

LaBerge, D. (2002). Attentional control: Brief and prolonged. Psychological Research, 66, 220-223.

Lang, P. J., Bradley, M. M., \& Cuthbert, B. N. (1997). Motivated attention: Affect, activation, and action. In P. J. Lang, R. F. Simons, \& M. T. Balaban (Eds.), Attention and orienting: Sensory and motivational processes (pp. 97-135). Hillsdale, NJ: Erlbaum.

Lang, P. J., Davis, M., \& Öhman, A, (2000). Fear and anxiety: Animal models and human cognitive psychopharmacology. Journal of Affective Disorders, 61, 137-159. 


\section{References}

Lavy, E. H. (1993). Attentional bias and anxiety: Conceptual issues and empirical data. Unpublished doctoral dissertation, Rijksuniversiteit Limburg, Maastricht, The Netherlands.

Lavy, E., Van den Hout, M., \& Arntz, A. (1993). Attentional bias and facilitated escape: A pictorial test. Advances in Behaviour Research and Therapy, 15, 279-289.

Lazarus, R. S. (1984). On the primacy of cognition. American Psychologist, 39, 124-129.

LeDoux, J. E. (1992). Emotion and the amygdala. In J. P. Aggleton (Ed.), The amygdala: Neurobiological aspects of emotion, memory, and mental dysfunction (pp. 339-351). New York, NY: Wiley-Liss.

LeDoux, J. E. (1993). Cognition versus emotion, again this time in the brain: A response to Parrott and Schulkin. Cognition and Emotion, 7, 61-64.

LeDoux, J. E. (1995). In search of an emotional system in the brain: Leaping from fear to emotion and consciousness. In M. S. Gazzaniga. (Ed.), The cognitive neurosciences (pp. 1049 1061). Cambridge, MA, US: The MIT Press.

LeDoux, J. E. (1996). The emotional brain. New York: Simon \& Schuster.

Lees, A., Mogg, K., \& Bradley, B. P. (2005). Health anxiety, anxiety sensitivity, and attentional biases for pictorial and linguistic health-threat cues. Cognition and Emotion, 19, 453-462.

Lipp, O. V., \& Derakshan, N. (2005). Attentional bias to pictures of fear-relevant animals in a dot probe task. Emotion, 5, 365-369.

Lipp, O. V., Derakshan, N., Waters, A. M., \& Logies, S. (2004). Snakes and cats in the flower bed: fast detection is not specific to pictures of fear-relevant animals. Emotion, 4, 233-250.

Liu, X., Qian, M., Zhou, X., \& Wang, A. (2006). Repeating the stimulus exposure to investigate what happens after initial selective attention to threatening pictures. Personality and Individual Differences, 40, 1007-1016.

Logan, G. D. (1992). Attention and preattention in theories of automaticity. American Journal of Psychology, 105, 317-339.

Lu, C. H., \& Proctor, R. W. (1995). The influence of irrelevant location information on performance: A review of the Simon and spatial Stroop effects. Psychonomic Bulletin and Review, 2, 174-207.

Luck, S. J., \& Hillyard, S. A. (1994). Spatial filtering during visual search: Evidence from human electrophysiology. Journal of Experimental Psychology: Human Perception and Performance, 20, 1000-1014.

Luck, S. J., \& Vecera, S. P. (2002). Attention. In H. Pashler, \& S. Yantis (Eds.), Steven's handbook of experimental psychology ( $3^{\text {rd }}$ Ed.), Vol. 1: Sensation and perception. (pp. 235-286). New York, NY, US: John Wiley \& Sons, Inc.

Luecken, L. J., Tartaro, J., \& Appelhans, B. (2004). Strategic coping responses and attentional biases. Cognitive Therapy and Research, 28, 23-37.

M

Mackintosh, B., \& Mathews, A. (2003). Don't look now: Attentional avoidance of emotionally valenced cues. Cognition and Emotion, 17, 623-646.

MacLeod, C. M. (1991). Half a century on the Stroop effect: An integrative review. Psychological Bulletin, 109, 163-203.

MacLeod, C. (2005). The Stroop task in clinical research. In D. C. Rubin \& A. Wenzel (Eds.), Cognitive methods and their application to clinical research (Vol. ix, pp. 41-62). Washington, DC, US: American Psychological Association.

MacLeod, C., Campbell, L., Rutherford, E., \& Wilson, E. (2004). The causal status of anxiety-linked attentional and interpretive bias. In J. Yiend (Ed.), Cognition, emotion and psychopathology: Theoretical, empirical and clinical directions (pp. 172-189). Cambridge, UK: Cambridge University Press.

MacLeod, C., \& Hagan, R. (1992). Individual differences in the selective processing of threatening information, and emotional responses to a stressful life event. Behaviour Research \& Therapy, 30, 151161.

MacLeod, C., \& Mathews, A. (1988). Anxiety and the allocation of attention to threat. Quarterly Journal of Experimental Psychology: Human Experimental Psychology, 40, 653-670.

MacLeod, C., Mathews, A., \& Tata, P. (1986). Attentional bias in emotional disorders. Journal of Abnormal Psychology, 95, 15-20.

MacLeod, C., \& Rutherford, E. M. (1992). Anxiety and the selective processing of emotional information: Mediating roles of awareness, trait and state variables, and personal relevance of stimulus materials. 
Behaviour Research and Therapy, 30, 479-491.

MacLeod, C., Rutherford, E., Campbell, L., Ebsworthy, G., \& Holker, L. (2002). Selective attention and emotional vulnerability: assessing the causal basis of their association through the experimental manipulation of attentional bias. Journal of Abnormal Psychology, 111, 107-123.

Mansell, W., Clark, D. M., Ehlers, A., \& Chen, Y. P. (1999). Social anxiety and attention away from emotional faces. Cognition and Emotion, 13, 673-690.

Mansell, W., Ehlers, A., Clark, D. M., \& Chen, Y. P. (2002). Attention to positive and negative socialevaluative words: Investigating the effects of social anxiety, trait anxiety and social-threat. Anxiety, Stress and Coping: An International Journal, 15, 19-29.

Marcel, A. J. (1983). Conscious and unconscious perception: Experiments on visual masking and word recognition. Cognitive Psychology, 15, 197-237.

Markman, A. B., \& Brendl, M. (2005). Constraining theories of embodied cognition. Psychological Science, 16, 6-10.

Marshall, A. (Producer), \& Harlin, R. (Director). (1993). Cliffhanger [Motion picture, 4:00-10:50]. United States: Columbia Tristar.

Martin, M., Williamson, D. A., \& Clark, D. M. (1991). Does anxiety lead to selective processing of threatrelated information? Behaviour Research and Therapy, 29, 147-160.

Mathews, A. (2004). On the malleability of emotional encoding. Behaviour Research and Therapy, 42, 10191036.

Mathews, A., \& Klug, F. (1993). Emotionality and interference with color-naming in anxiety. Behaviour Research and Therapy, 31, 57-62.

Mathews, A., \& Mackintosh, B. (1998). A cognitive model of selective processing in anxiety. Cognitive Therapy and Research, 22, 539-560.

Mathews, A., \& MacLeod, C. (1985). Selective processing of threat cues in anxiety states. Behaviour Research and Therapy, 23, 563-569.

Mathews, A., \& MacLeod, C. (1994). Cognitive approaches to emotion and emotional disorders. Annual Review of Psychology, 45, 25-50.

Mathews, A., \& MacLeod, C. (2002). Induced emotional biases have causal effects on anxiety. Cognition and Emotion, 16, 310-315.

Mathews, A., \& MacLeod, C. (2005). Cognitive vulnerability to emotional disorders. Annual Review of Clinical Psychology, 1, 7.1-7.29.

Mathews, A., May, J., Mogg, K., \& Eysenck, M. (1990). Attentional bias in anxiety: Selective search or defective filtering? Journal of Abnormal Psychology, 99, 166-173.

Mathews, A., Mogg, K., Kentish, J., \& Eysenck, M. (1995). Effect of psychological treatment on cognitive bias in generalized anxiety disorder. Behaviour Research and Therapy, 33, 293-303.

Mathews, A., Ridgeway, V., \& Williamson, D. A. (1996). Evidence for attention to threatening stimuli in depression. Behaviour Research and Therapy, 34, 695-705.

Mathews, A., \& Sebastian, S. (1993). Suppression of emotional Stroop effects by fear-arousal. Cognition and Emotion, 7, 517-530.

Mattia, J. I., Heimberg, R. G., \& Hope, D. A. (1993). The revised Stroop color-naming Task in social phobias. Behaviour Research and Therapy, 31, 305-313.

Matthews, G., \& Wells, A. (2000). Attention, automaticity, and affective disorder. Behavior Modification, 24, 69-93.

Mayer, B., Merckelbach, H., De Jong, P. J., \& Leeuw, I. (1999). Skin conductance responses of spider phobics to backwardly masked phobic cues. Journal of Psychophysiology, 13, 152-159.

McAuliffe, J., \& Pratt, J. (2005). The role of temporal and spatial factors in the covert orienting of visual attention tasks. Psychological Research, 69, 285-291.

McKenna, F. P., \& Sharma, D. (2004). Reversing the emotional Stroop effect reveals that it is not what it seems: The role of fast and slow components. Journal of Experimental Psychology: Learning, Memory, and Cognition, 30, 382-392.

McNally, R. J. (1995). Automaticity and the anxiety disorders. Behaviour Research and Therapy, 33, 747-754.

McNally, R. J., Kaspi, S. P., Riemann, B. C., \& Zeitlin, S. B. (1990). Selective processing of threat cues in Posttraumatic stress disorder. Journal of Abnormal Psychology, 99, 398-402.

Merikle, P. M. (1992). Perception without awareness: Critical issues. American Psychologist, 47, 792-795. 


\section{References}

Merikle, P. M., Smilek, D., \& Eastwood, J. D. (2001). Perception without awareness: Perspectives from Cognitive psychology. Cognition, 79, 115-134.

Mogg, K., \& Bradley, B. P. (1998). A cognitive-motivational analysis of anxiety. Behaviour Research and Therapy, 6, 809-848.

Mogg, K., \& Bradley, B. P. (1999a). Some methodological issues in assessing attentional biases for threatening faces in anxiety: A replication study using a modified version of the probe detection task. Behaviour Research and Therapy, 37, 595-604.

Mogg, K., \& Bradley, B. P. (1999b). Orienting of attention to threatening facial expressions presented under conditions of restricted awareness. Cognition and Emotion, 13, 713-740.

Mogg, K., \& Bradley, B. P. (2002). Selective orienting of attention to masked threat faces in social anxiety. Behaviour Research and Therapy, 40, 1403-1414.

Mogg, K., \& Bradley, B. (2004). A cognitive-motivational perspective on the processing of threat information and anxiety. In J. Yiend (Ed.), Cognition, emotion and psychopathology: Theoretical, empirical and clinical directions (pp. 68-85). Cambridge, UK: Cambridge University Press.

Mogg, K., \& Bradley, B. P. (2005). Attentional bias in generalized anxiety disorder versus depressive disorder. Cognitive Therapy and Research, 29, 29-45.

Mogg, K., \& Bradley, B. P. (2006). Time course of attentional bias for fear-relevant pictures in spider-fearful individuals. Behaviour Research and Therapy, 44, 1241-1250.

Mogg, K., Bradley, B. P., De Bono, J., \& Painter, M. (1997). Time course of attentional bias for threat information in non-clinical anxiety. Behaviour Research and Therapy, 35, 297-303.

Mogg, K., Bradley, B. P., Dixon, C., Fisher, S., Twelftree, H., \& McWilliams, A. (2000). Trait anxiety, defensiveness and selective processing of threat: An investigation using two measures of attentional bias. Personality and Individual Differences, 28, 1063-1077.

Mogg, K., Bradley, B. P., \& Hallowell, N. (1994). Attentional bias to threat: Roles of trait anxiety, stressful events, and awareness. Quarterly Journal of Experimental Psychology: Human Experimental Psychology, 47a, 841-864.

Mogg, K., Bradley, B. P., Miles, F., \& Dixon, R. (2004). Time course of attentional bias for threat scenes: Testing the vigilance-avoidance hypothesis. Cognition and Emotion, 18, 689-700.

Mogg, K., Bradley, B. P., \& Williams, R. (1995). Attentional bias in anxiety and depression: The role of awareness. British Journal of Clinical Psychology, 34, 17-36.

Mogg, K., Bradley, B. P., Williams, R., \& Mathews, A. (1993). Subliminal processing of emotional information in anxiety and depression. Journal of Abnormal Psychology, 102, 304-311.

Mogg, K., Kentish, J., \& Bradley, B. P. (1993). Effects of anxiety and awareness on colour identification latencies for emotional words. Behaviour Research and Therapy, 31, 559-567.

Mogg, K., Mathews, A., Bird, C., \& MacGregor-Morris, R. (1990). Effects of stress and anxiety on the processing of threat stimuli. Journal of personality and Social Psychology, 59, 1230-1237.

Mogg, K., Mathews, A., \& Eysenck, M. (1992). Attentional bias to threat in clinical anxiety states. Cognition and Emotion, 6, 149-159.

Mogg, K., Mathews, A., May, J., Grove, M., Eysenck, M., \& Weinman, J. (1991). Assessment of cognitive bias in anxiety and depression using a colour perception task. Cognition and Emotion, 5, 221-238.

Mogg, K., Mathews, A., \& Weinman, J. (1987). Memory bias in clinical anxiety. Journal of Abnormal Psychology, 96, 94-98.

Mogg, K., Mathews, A., \& Weinman, J. (1989). Selective processing of threat cues in clinical anxiety states: A replication. Behaviour research and Therapy, 27, 317-323.

Mogg, K., McNamara, J., Powys, M., Rawlinson, H., Seiffer, A., \& Bradley, B. P. (2000). Selective attention to threat: A test of two cognitive models of anxiety. Cognition and Emotion, 14, 375-399.

Mogg, K., Millar, N., \& Bradley, B. P. (2000). Biases in eye movements to threatening facial expressions in generalized anxiety disorder and depressive disorder. Journal of Abnormal Psychology, 109, 695-704.

Mogg, K., Philippot, P., \& Bradley, B. P. (2004). Selective Attention to Angry Faces in Clinical Social Phobia. Journal of Abnormal Psychology, 113, 160-165.

Morris, J. S., Öhman, A., \& Dolan, R. J. (1998). Conscious and unconscious emotional learning in the human amygdala. Nature, 393, 467-470.

Morris, J. S., Öhman, A., \& Dolan, R. J. (1999). A subcortical pathway to the right amygdala mediating "unseen" fear. Proceedings of the National Academy of Sciences, 96, 1680-1685. 
Muris, P., \& Merckelbach, H. (1996). A comparison of two spider fear questionnaires. Journal of Behavior Therapy and Experimental Psychiatry, 27, 241-244.

Muris, P., Merckelbach, H., \& de Jong, A. (1995). Colour-naming of dentist-related words: Role of coping style, dental anxiety, and trait anxiety. Personality and Individual Differences, 18, 685-688.

Musa, C., Lépine, J. P., Clark, D. M., Mansell, W., \& Ehlers, A. (2003). Selective attention in social phobia and the moderating effect of a concurrent depressive disorder. Behaviour Research and Therapy, 41, 10431054 .

N

Neumann, O. (1990). Visual attention and action. In O. Neumann \& W. Prinz (Eds.), Relationships between perception and action: Current approaches (pp. 227-267). Berlin: Springer-Verlag.

Neumann, R., Förster, J., \& Strack, F. (2003). Motor compatibility: The bidirectional link between behavior and evaluation. In J. Musch, \& K. C. Klauer (Eds.), The psychology of evaluation: Affective processes in cognition and emotion (pp. 371-391). Mahwah, NJ: Lawrence Erlbaum Associates.

Nicoletti, R., \& Umiltà, C. (1984). Right-left prevalence in spatial compatibility. Perception \& Psychophysics, 35, 333-343.

Nicoletti, R., \& Umiltà, C. (1989). Splitting visual space with attention. Journal of Experimental Psychology: Human Perception and Performance, 15, 164-169.

O

Oatley, K., \& Johnson-Laird, P. (1987). Towards a cognitive theory of emotions. Cognition and Emotion, 1, $29-50$.

Öhman, A. (1993). Fear and anxiety as emotional phenomena: clinical phenomenology, evolutionary perspective, and information processing mechanisms. In M. Lewis \& J. M. Haviland (Eds.), Handbook of Emotions. New York: Guilford.

Öhman, A. (1994). The psychophysiology of emotion: Evolutionary and non-conscious origins. In G. d'Ydewalle, P. Eelen et al. (Eds.), International perspectives on psychological science: The state of the art. (Vol.2, p. 197-227). Hillsdale, NJ: Lawrence Erlbaum Associates.

Öhman, A. (1996). Preferential preattentive processing of threat in anxiety: Preparedness and attentional biases. In R. M. Rapee (Ed.), Current controversies in the anxiety disorders. New York: Guilford Press.

Öhman, A. (2000). Fear and anxiety: Evolutionary, cognitive and clinical perspectives. In M. Lewis \& J. M. Haviland (Eds.), Handbook of emotions ( $2^{\text {nd }}$ Ed., pp. 573-593). New York: Guilford Press.

Öhman, A., Flykt, A., \& Esteves, F. (2001). Emotion drives attention: Detecting the snake in the grass. Journal of Experimental Psychology: General, 130, 466-478.

Öhman, A., Lundqvist, D., \& Esteves, F. (2001). The face in the crowd revisited: A threat advantage with schematic stimuli. Journal of Personality and Social Psychology, 80, 381-396.

Öhman, A., \& Mineka, S. (2001). Fears, phobias, and preparedness: Toward an evolved module of fear and fear learning. Psychological Review, 108, 483-522.

Öhman, A., \& Soares, J. J. F. (1993). On the automatic nature of phobic fear: Conditioned electrodermal responses to masked fear-relevant stimuli. Journal of Abnormal Psychology, 102, 121-132.

Öhman, A., \& Soares, J. J .F. (1994). "Unconscious anxiety”: Phobic responses to masked stimuli. Journal of Abnormal Psychology, 103, 231-240.

Öhman, A., \& Soares, J. J. F. (1998). Emotional conditioning to masked stimuli: Expectancies for aversive outcomes following nonrecognized fear-relevant stimuli. Journal of Experimental Psychology: General, 127, 69-82.

P

Pashler, H. (1998). Attention. Hove, England: Psychology Press/Erlbaum (UK) Taylor \& Francis.

Pessoa, L., Japee, S., \& Ungerleider, L. G. (2005). Visual Awareness and the Detection of Fearful Faces. Emotion, 5, 243-247.

Phaf, R. H., \& Kan, K.-J. (in press). The automaticity of emotional Stroop: A meta-analysis. Journal of Behavior Therapy and Experimental Psychiatry.

Pineles, S. L., \& Mineka, S. (2005). Attentional biases to internal and external sources of potential threat in social anxiety. Journal of Abnormal Psychology, 114, 314-318.

Posner, M. I. (1980). Orienting of attention. Quarterly Journal of Experimental Psychology, 32, 3-25. 


\section{References}

Posner, M. I., \& Cohen, Y. (1984). Components of visual orienting. In H. Bouma \& D. G. Bouwhuis (Eds.), Attention and performance X: Control of language processes (pp. 531-556). Hillsdale, NJ: Erlbaum.

Posner, M. I., \& Petersen, S. E. (1990). The attention system of the human brain. Annual Review of Neuroscience, 13, 25-42.

Posner, M. I., \& Rothbart, M. K. (2005). Influencing brain networks: Implications for education. Trends in Cognitive Sciences, 9, 99-103.

Posner, M. I., Snyder, C. R., \& Davidson, B. J. (1980). Attention and the detection of signals. Journal of Experimental Psychology: General, 109, 160-174.

Pratto, F., \& John, O. P. (1991). Automatic vigilance: The attention-grabbing power of negative social information. Journal of Personality and Social Psychology, 61, 380-391.

Purcell, D. G., Stewart, A. L., \& Skov, R. B. (1996). It takes a confounded face to pop out of a crowd. Perception, 25, 1091-1108.

$\mathrm{R}$

Rafal, R., \& Henik, A. (1994). The neurology of inhibition: Integrating controlled and automatic processes. In D. Dagenbach \& C. H. Thomas (Eds.), Inhibitory processes in attention, memory, and language (pp. 151). San Diego: Academic Press.

Ratcliff, R. (1979). Group reaction time distributions and an analysis of distribution statistics. Psychological Bulletin, 86, 446-461.

Richards, A., \& French, C. C. (1992). An anxiety-related bias in semantic activation when processing threat/neutral homographs. Quarterly Journal of Experimental Psychology, 45, 503-525.

Richards, A., French, C. C., Johnsson, W., Naparstek, J., \& Williams, J. (1992). Effects of mood manipulation and anxiety on performance of an emotional Stroop task. British Journal of Psychology, 83, 479-491.

Ridderinkhof, K. R. (2002). Activation and suppression in conflict tasks: Empirical clarification through distributional analyses. In W. Prinz \& B. Hommel (Eds.), Common Mechanisms in Perception and Action. Attention \& Performance (Vol. XIX, pp. 494-519). Oxford: Oxford University Press.

Rinck, M., \& Becker, E. S. (2005). A comparison of attentional biases and memory biases in women with social phobia and major depression. Journal of Abnormal Psychology, 114, 62-74.

Rinck, M., \& Becker, E. (in press). Approach and avoidance in fear of spiders. Journal of Behaviour Therapy and Experimental Psychiatry.

Rinck, M., Becker, E. S., Kellermann, J., \& Roth, W. T. (2003). Selective attention in anxiety: Distraction and enhancement in visual search. Depression and Anxiety, 18, 18-28.

Rinck, M., Reinecke, A., Ellwart, T., Heuer, K., \& Becker, E. S. (2005). Speeded detection and increased distraction in fear of spiders: Evidence from eye movements. Journal of Abnormal Psychology, 114, 235224.

Rizzolatti, G., Riggio, L., \& Sheliga, B. M. (1994). Space and selective attention. In C. Umilta \& M. Moscovitch (Eds.), Attention and performance 15: Conscious and nonconscious information processing (pp. 232-265). Cambridge, MA: The MIT Press.

Rohner, J. C. (2002). The time-course of visual threat processing: High trait anxious individuals eventually avert their gaze from angry faces. Cognition and Emotion, 16, 837-844.

Rotteveel, M., \& Phaf, R. H. (2004). Automatic affective evaluation does not automatically predispose for arm flexion and extension. Emotion, 4, 156-172.

Russell, J. A., \& Barrett, L. F. (1999). Core affect, prototypical emotional episodes, and other things called emotion: Dissecting the elephant. Journal of Personality and Social Psychology, 76, 805-819.

S

Salemink, E., van den Hout, M. A., \& Kindt, M. (2007). Selective attention and threat: Quick orienting versus slow disengagement and two versions of the dot probe Task. Behaviour Research and Therapy, 45, 607-615.

Schmukle, S., C. (2005). Unreliability of the dot probe task. European Journal of Personality, 19, 595-605.

Schrooten, M. G. S., \& Smulders, F. T. Y. (2007a). A Simon effect for threat-related stimulus content. Journal of Behavior Therapy and Experimental Psychiatry, 38, 121-132. [Chapter 3]

Schrooten, M. G. S., \& Smulders, F. T. Y. (2007b). Biased processing of emotional information in anxiety: Evidence from Simon effects. Manuscript submitted for publication. [Chapter 4] 
Schweickert, R. (1985). Separable effects of factors on speed and accuracy: Memory scanning, lexical decision, and choice tasks. Psychological Bulletin, 97, 530-546.

Seligman, M. E. P. (1971), Phobias and preparedness. Behavior Therapy, 2, 307-320.

Shiffrin, R. M., \& Schneider, W. (1977). Controlled and automatic human processing: Perceptual learning, automatic attending, and a general theory. Psychological Review, 84, 127-190.

Simon, J. R. (1990). The effects of an irrelevant directional cue on human information processing. In R. W.

Proctor \& T. G. Reeve (Eds.), Stimulus response compatibility: An integrated perspective. Advances in psychology (Vol. 65, pp. 31-86). Oxford, England: North Holland.

Simon, J. R., Acosta, E., Mewaldt, S. P., \& Speidel, C. R. (1976). The effect of an irrelevant directional cue on choice reaction time: Duration of the phenomenon and its relation to stages of processing. Perception and Psychophysics, 19, 16-22.

Snodgrass, M., Bernat, E., \& Shevrin, H. (2004). Unconscious perception: A model-based approach to method and evidence. Perception and Psychophysics. 66, 846-867.

Sobotka, S. S., Davidson, R. J., \& Senulis, J. A. (1992). Anterior brain electrical asymmetries in response to reward and punishment. Electroencephalography and Clinical Neurophysiology, 83, 236-247.

Solarz, A. K. (1960). Latency of instrumental responses as a function of compatibility with the meaning of eliciting verbal signs. Journal of Experimental Psychology, 59, 239-245.

Spielberger, C. D. (1972). Anxiety: Current Trends in Theory and Research, Vol. 1. London: Academic Press.

Spielberger, C. D., Gorsuch, R., \& Lushene, R. (1970). The State-Trait Anxiety Inventory (STAI) Test Manual Form X. Palo Alto, Calif.: Consulting Psychologists press.

Stormark, K. M., \& Hugdahl, K. (1996). Peripheral cuing of covert spatial attention before and after emotional conditioning of the cue. International Journal of Neuroscience, 86, 225-240.

Stormark, K. M., Hugdahl, K., \& Posner, M. I. (1999). Emotional modulation of attention orienting: A classical conditioning study. Scandinavian Journal of Psychophysiology, 40, 91-99.

Stormark, K. M., Nordby, H., \& Hugdahl, K. (1995). Attentional shifts to emotionally charged cues: Behavioural and ERP data. Cognition and Emotion, 9, 507-523.

Stroop, J. R. (1935). Studies of interference in serial verbal reactions. Journal of Experimental Psychology, 18 , 643-662.

Szymanski, J., \& O'Donohue, W. (1995). Fear of spiders questionnaire. Journal of Behavior Therapy and Experimental Psychiatry, 26, 31-34.

$\mathrm{T}$

Tata, P. R., Leibowitz, J. A., Prunty, M. J., Cameron, M., \& Pickering, A. D. (1996). Attentional bias in Obsessional Compulsive Disorder. Behaviour Research and Therapy, 34, 53-60.

Tipples, J., Young, A. W., Quinlan, P., Broks, P., \& Ellis A. W. (2002). Searching for threat. Quarterly Journal of Experimental Psychology, 55, 1007-1026.

Treisman, A., Vieira, A., \& Hayes, A. (1992). Automaticity and preattentive processing. American Journal of Psychology, 105, 341-362.

V

Van Damme, S., Crombez, G., Hermans, D., Koster, E. H. W., \& Eccleston, C. (2006). The role of extinction and reinstatement in attentional bias to threat: A conditioning approach. Behaviour Research and Therapy, 44, 1555-1563.

Van der Ploeg, H. M., Defares, P. B., \& Spielberger, C. D. (1980). Handleiding bij de Zelf-Beoordelings Vragenlijst: Een Nederlandstalige bewerking van de Spielberger State-Trait Anxiety Inventory [A manual for the Self-Assessment Questionnaire: A Dutch version of the Spielberger State-Trait Anxiety Inventory]. Swets \& Zeitlinger b.v., Lisse.

Van Honk, J., Tuiten, A., De Haan, E., Van den Hout, M., \& Stam, H. (2001). Attentional bias for angry faces: Relationships to trait anger and anxiety. Cognition and Emotion, 15, 279-297.

Van Rijsoort, S., Vervaeke, G., \& Emmelkamp, P. (1997). The Penn State Worry Questionnaire en de Worry Domains Questionnaire: eerste resultaten bij een normale Nederlandse populatie [The Penn State Worry Questionnaire and the Worry Domains Questionnaire: first results in a normal Dutch population]. Gedragstherapie, 30, 121-128.

Van Rijsoort, S., Emmelkamp, P., \& Vervaeke, G. (1999). The Penn State Worry Questionnaire en de Worry 


\section{References}

Domains Questionnaire: structure, reliability and validity. Clinical Psychology and Psychotherapy, 6, $297-$ 307.

Vorberg, D., Mattler, U., Heinecke, A., Schmidt, T., \& Schwarzbach, J. (2002). Different time courses for visual perception and action priming. Proceedings of the National Academy of Sciences, 100, 6275-6280.

W

Wald, F.D.M., Mellenbergh, G.J. (1990). De verkorte versie van de Nederlandse vertaling van de Profile of Mood State (POMS). [The Dutch shortened version of the Profile of Mood State (POMS).]. Nederlands Tijdschrift van de Psychologie en haar Grensgebieden, 45, 86-91.

Walters, G. (Producer), \& Unkrich, L. (Director). (2003). Finding Nemo [Motion Picture, 5:04-11:15]. United States: Walt Disney/ Pixar.

Wascher, E., \& Wauschkuhn, B. (1996). The interaction of stimulus- and response-related processes measured by event-related lateralizations of the EEG. Electroencephalography and Clinical Neurophysiology, 99, 149162.

Waters, A. M., Nitz, A. B., Craske, M. G., \& Johnson, C. (2007). The effects of anxiety upon attention allocation to affective stimuli. Behaviour Research and Therapy, 45, 763-774.

Watts, F. N., McKenna, F. P., Sharrock, R., \& Trezise, L. (1986). Colour-naming of phobia-related words. British Journal of Psychology, 77, 97-108.

Weertman, A., Arntz, A., \& Kerkhofs, M. L. M. (2000). Gestructureerd klinisch interview voor DSM-IV'as-II persoonlijkheidsstoornissen (SCID-II) [Structured clinical interview for DSM-IV axis II personality disorders (SCID-II)]. Lisse, Netherlands: Swets \& Zeitlinger.

Weinberger, D. A., Schwartz, G. E., \& Davidson, J. R. (1979). Low-anxious, high-anxious, and repressive coping styles: Psychometric patterns and behavioural and physiological responses to threat. Journal of Abnormal Psychology, 88, 369-380.

Wells, A., \& Matthews, G. (1994). Attention and emotion: A clinical perspective. Hove, UK: Erlbaum.

Wentura, D., Rothermund, K., \& Bak, P. (2000). Automatic Vigilance: The attention-grabbing power of approach- and avoidance-related social information. Journal of Personality and Social Psychology, 78, 1024-1037.

Wenzel, A., \& Holt, C. S. (1999). Dot probe performance in two specific phobias. British Journal of Clinical Psychology, 38, 407-410.

Whalen, P. J., Rauch, S. L., Etcoff, N. L., McInerney, S. C., Lee, M. B., \& Jenike, M. A. (1998). Masked presentations of emotional facial expressions modulate amygdala activity without explicit knowledge. The Journal of Neuroscience, 18, 411-418.

Williams, J. M. G., Mathews, A., \& MacLeod, C. (1996). The emotional Stroop task and psychopathology. Psychological Bulletin, 120, 3-24.

Williams, J. M. G., Watts, F. N., MacLeod, C., \& Mathews, A. (1988). Cognitive psychology and emotional disorders. Chichester, UK: Wiley.

Williams, J. M. G., Watts, F. N., MacLeod, C., \& Mathews, A. (1997). Cognitive psychology and emotional disorders ( $2^{\text {nd }}$ Ed.). Chichester, UK: Wiley.

Wilson, E., \& MacLeod, C. (2003). Contrasting two accounts of anxiety-linked attentional bias: Selective attention to varying levels of stimulus threat intensity. Journal of Abnormal Psychology, 112, 212-218.

Wolfe, J. M. (1998). Visual search. In H. Pashler (Ed.). Attention. (pp. 13-73). Hove, England: Psychology Press/Erlbaum (UK.) Taylor \& Francis.

Woodman, G. F. \& Luck, S. J. (1999). Electrophysiological measurement of rapid shifts of attention during visual search, Nature, 400, 867-869.

Y

Yantis, S. (1996). Control of visual attention. In H. Pashler (Ed.), Attention (p.223-256). San Diego: Psychology Press.

Yiend, J. (Ed.). (2004a). Cognition, emotion and psychopathology: Theoretical, empirical and clinical directions. Cambridge, UK: Cambridge University Press.

Yiend, J. (2004b). An introduction and synthesis. In J. Yiend (Ed.), Cognition, emotion and psychopathology: Theoretical, empirical and clinical directions (pp. 1-7). Cambridge, UK: Cambridge University Press.

Yiend, J. \& Mackintosh, B. (2004). The experimental modification of processing biases. In J. Yiend (Ed.), Cognition, emotion and psychopathology: Theoretical, empirical and clinical directions (pp. 190-210). 
Cambridge, UK: Cambridge University Press.

Yiend, J., \& Mathews, A. (2001). Anxiety and attention to threatening pictures. Quarterly Journal of Experimental Psychology, 54A, 665-681.

Z

Zajonc, R. B. (1984). On the primacy of affect. American Psychologist, 39, 117-123. 


\section{Dankwoord}

Dank aan: Fren Smulders (co-promotor) voor het project, de kennis, de brain Olympics en de mooie manier van denken; Harald Merckelbach (promotor) en Anita Jansen (capgroepvoorzitter) voor het advies en de daadkracht op cruciale momenten; Arnoud Arntz voor het commentaar en de suggesties in verband met o.a. de klinische studie; RIAGG Maastricht voor de samenwerking; Gerard van Breukelen voor het scherpe statistisch advies wanneer ik kwam aanzetten met mijn multi-factoriële designs; de secretaresses Jessie Beerthuyzen, Truus van Uijen en Nicole Niering; Ron Hellenbrand, Jacco Ronner en Johan Gielissen voor al de technische ondersteuning ("If you've got a problem and no one else can help, and if you can find them, maybe you can hire... the 3-Team", merci); Candy Gijsen, Janneke Giesen, Fatma Al-Harazi, Nicolette Siep, Sven Akkerman, Karin Bijl en Marinka Reubzaet voor de hulp bij data-verzameling; Thamare van Roosmalen, Rozan Souren en de andere onderzoeksassistentes van RIAGG Maastricht voor de werving van de angstige klinische groep; de vele niet-kleurenblinde proefpersonen; collega's van toen nog Experimentele Psychologie; Reinout Wiers, Fren Smulders, Rob Ruiter, Anne Roefs, Ellen Jongen, Tim Schoenmakers, Katrijn Houben en Loes Kessels, kortom de Attimpiërs voor de discussie, aandacht, attitude en im/explicietheid; Janneke Giesen voor al de getoonde interesse en het niet aflatend enthousiasme over de SAST en de RAT; Ellen Jongen (paranimf) voor al die vriendschap sinds the Butcher Boy, de droge humor, de steun, het lezen van mijn stukken, haar inzicht in ons vakgebied en haar inzichten in mij; Anika Dam (paranimf) en Raoul Martin voor de gesprekken, de vriendschap, de slaapplek en the daring concepts (Anika, Renée, zusterlief, stelling: ook zonder bloedband kan men zusters voor het leven zijn); Marieke Werrij voor de gezelligheid, het gevoel van thuiskomen en het meetorsen van de laatste loodjes; Anne Roefs alias Doctor Rufus en Pascal van Gerven vooral, maar niet alleen, voor het vertrouwen in mijn kunnen en de mental coaching ("I used to have doubts, but now I'm not sure" remember in Mahl @ Coffee Lovers); Ramona Guerrieri voor de koffieklatch, de brainstorm en de pizza's op momenten van troost; Elke Smeets voor de positive vibes en de Belgische drugs - ze hielpen; de andere Belgen op de derde zodat ik mijn roots toch maar niet zou vergeten; Judith Peters voor de aanmoediging; Irma Baars voor de girl power en Het back-up plan; en mijn andere Partners in Drama; Lonneke Koudijs, Dyon Scheijen, Claudia Steinbusch, Thijs Thielemans, Karian van Ginkel, Sander Ubbink en Ger Essers, kortom de lopers / stappers voor de vele kilometers en de afleiding ("Ut kump wie ut kump, ut geit wie ut geit, ut lup wie ut lup en ut is wie ut is"); liebe Leute heute in Deutschland; mama, papa en Philip voor de courage, de liefde en het tegenwicht - merci.

Martien

Maastricht, mei 2007 


\section{Curriculum Vitae}

Martien Germaine Silvie Schrooten was born in Bree (Belgium) on June 22, 1978. In 1996 she graduated from secondary school, Agnetendal in Peer (Belgium). In 1997, after having studied classical philology for one year at the Katholieke Universiteit Leuven (Belgium), she started her study psychology at the Universiteit Maastricht (the Netherlands). She performed a research internship in the Language and Music Group at the Center for Research in Cognitive Neurosciences (CRNCCNRS) in Marseille (France). In October 2001 she received her master's degree in Biopsychology (Neuropsychology). During her study psychology, she also worked as a teaching and research assistant at the Universiteit Maastricht. From October 2001 until February 2002 she was employed as research assistant at the Max Planck Institute for Human Cognitive and Brain Science in Leipzig (Germany). In September 2002 she started her $\mathrm{PhD}(\mathrm{AiO})$ project at the department of Experimental Psychology, Universiteit Maastricht, resulting in this thesis. Since March 2007 she is employed as statistical researcher at Statistics Netherlands (CBS), Division of Social and Spatial Statistics, in Heerlen (the Netherlands).

\section{Publications}

Schrooten, M.G.S., \& Smulders, F.T.Y. (2004). Een experimentele kijk op angst: aandacht en automatisme in de verwerking van bedreigende stimuli. [An experimental approach to anxiety: Attention and automatism in the processing of threatening stimuli.] Nederlands Tijdschrift voor de Psychologie en haar Grensgebieden, 59, 135-149.

Schrooten, M.G.S., \& Smulders, F.T.Y. (2007). A Simon effect for threat-related stimulus content. Journal of Behavior Therapy and Experimental Psychiatry, 38, 121-132.

Schrooten, M.G.S., \& Smulders, F.T.Y. (submitted). Anxiety-related attentional bias and spatial response bias independently assessed by a probe-classification task.

Schrooten, M.G.S., \& Smulders, F.T.Y. (submitted). Biased processing of emotional information in anxiety: Evidence from Simon effects.

Schrooten, M.G.S., \& Smulders, F. T. Y. (submitted). Temporal dynamics of selective attention in non-clinical anxiety.

Schrooten, M.G.S., Smulders, F.T.Y., \& Arntz, A. (submitted). Preferential processing of emotional stimulus content in pathological anxiety: Evidence from Simon effects.

Guerrieri, R., Nederkoorn, C., Schrooten, M., \& Jansen, A. (in preparation). How inducing impulsivity versus inhibition affects caloric intake in a sample of healthy women depends on dieting status, not restraint status. 


\section{Conference Presentations}

Schrooten, M.G.S., \& Smulders, F.T.Y (2004). Spatial response bias induced by emotional words: The spatial affective Simon task. Talk given at the 'Tagung experimentell arbeitender Psychologen', April 2004, Giessen, Germany.

Schrooten, M.G.S., \& Smulders, F.T.Y (2004). Spatial response bias induced by emotional words: The spatial affective Simon task. Poster presented at the open day of the Dutch Society of Psychonomics (NVP), Mai 2004, Maastricht, The Netherlands, and at seminar Neuroscience and Cognitive Control, December 2004, Ghent, Belgium.

Schrooten, M.G.S., \& Smulders, F.T.Y (2005). Anxiety-related attentional and spatial response bias: An electrophysiological approach (a). Poster presented at the 'Tagung experimentell arbeitender Psychologen', April 2005, Regensburg, Germany.

Schrooten, M.G.S., \& Smulders, F.T.Y (2005). Attentional and spatial affective response bias in anxiety: tapped at emotional stimulus onset and after delay (a). Poster presented at the EPOS/EPP/KLI Symposium 'Implicit cognition and attention in experimental psychopathology and health research: Indirect measures or dual processes?', August 2005, Maastricht, the Netherlands.

Schrooten, M.G.S., \& Smulders, F.T.Y (2005). Attentional bias and spatial response bias induced by emotional stimuli. Talk given at the EPOS/EPP/KLI Symposium 'Implicit cognition and attention in experimental psychopathology and health research: Indirect measures or dual processes?', August 2005, Maastricht, the Netherlands.

Schrooten, M.G.S., \& Smulders, F.T.Y (2005). Anxiety-related attentional and spatial response bias: An electrophysiological approach (b). Poster presented at the 45th Annual Meeting of the Society for Psychophysiological Research, September 2005, Lisbon, Portugal.

Schrooten, M.G.S., \& Smulders, F.T.Y (2005). Attentional and spatial affective response bias in anxiety: tapped at emotional stimulus onset and after delay (b). Poster presented at 2nd NWO (Netherlands Organization for Scientific Research) Summer School, October 2005, Doorwerth, the Netherlands.

Schrooten, M.G.S., \& Smulders, F.T.Y (2006). Anxiety-related attentional bias and spatial response bias: Their time-course revealed by distributional $R T$ analyses. Poster presented at the Third Belgian Meeting of Experimental Psychopathology, May 2006, Ghent, Belgium.

Schrooten, M.G.S., \& Smulders, F.T.Y (2006). Early preferential processing of threat: Evidence from Simon effects. Talk given at the EPOS symposium 'Affective modulation of cognitive performance', June 2006, Leiden, the Netherlands.

Schrooten, M.G.S., \& Smulders, F.T.Y (2006). Attentional bias in trait-anxiety: tapped at two moments in time-course, independent of exposure duration. Poster presented at the decennial jubilee symposium of Experimental Psychopathology Research School (EPP), October 2006, Den Bosch, the Netherlands. 